\title{
Antibiotics from Extremophilic Micromycetes
}

\author{
A. A. Baranova ${ }^{a}$, V. A. Alferova ${ }^{a, c}$, V. A. Korshun ${ }^{a, b, c}$, and A. P. Tyurin ${ }^{a, c, 1}$ \\ ${ }^{a}$ Gause Institute of New Antibiotics, Moscow, 119021 Russia \\ ${ }^{b}$ Shemyakin-Ovchinnikov Institute of Bioorganic Chemistry, Russian Academy of Sciences, Moscow, 117997 Russia \\ ${ }^{c}$ National Research University, Higher School of Economics, Moscow, 101000 Russia \\ Received April 4, 2020; revised May 8, 2020; accepted May 12, 2020
}

\begin{abstract}
Extremophilic microorganisms, which are capable of functioning normally at extremely high or low temperatures, pressure, and in other environmental conditions, have been in the focus of microbiologists' attention for several decades due to the biotechnological potential of enzymes inherent in extremophiles. These enzymes (also called extremozymes) are used in the production of food and detergents and other industries. At the same time, the inhabitants of extreme econiches remained almost unexplored for a long time in terms of the chemistry of natural compounds. In recent years, the emergence of new antibiotic-resistant strains of pathogens, which affect humans and animals has become a global problem. The problem is compounded by a strong slowdown in the development of new antibiotics. In search of new active substances and scaffolds for medical chemistry, researchers turn to unexplored natural sources. In recent years, there has been a sharp increase in the number of studies on secondary metabolites produced by extremophiles. From the discovery of penicillin to the present day, micromycetes, along with actinobacteria, are one of the most productive sources of antibiotic compounds for medicine and agriculture. Many authors consider extremophilic micromycetes as a promising source of small molecules with an unusual mechanism of action or significant structural novelty. This review summarizes the latest (for 2018-2019) experimental data on antibiotic compounds, which are produced by extremophilic micromycetes with various types of adaptation. Active metabolites are classified by the type of structure and biosynthetic origin. The data on the biological activity of the isolated metabolites are summarized.
\end{abstract}

Keywords: antibiotics, extremophiles, micromycetes, extreme habitats, resistance, biotechnology

DOI: $10.1134 / \mathrm{S} 1068162020060023$

\section{INTRODUCTION}

The resistance of pathogens to antimicrobial agents and the emergence of new pathogens, such as SARS$\mathrm{CoV}-2$, is a global threat to modern health. The problem caused by improper use and disposal of antimicrobial agents (for self-treatment, in animal husbandry, etc.) is compounded by a strong decline in the rate of development of new antibiotics because of their low investment returns $[1,2]$. The development of new anti-infectious agents and methods to combat the resistant microorganisms are a priority at both the international and national level. This problem is recorded in several political documents, i.e., UN Resolution A/RES/71/3 [3], the WHO Global action plan

Abbreviations: $\mathrm{AV}$, antiviral activity; $\mathrm{AB}$, antibacterial activity; $\mathrm{AF}$, antifungal activity; $\mathrm{CT}$, cytotoxic activity; AP, antiprotozoic activity; AT, antituberculosis activity; Ref., literature references (2018-2019); Cpd, serial number of the compound; MRSA, meticilllin-resistant Staphylococcus aureus; MRSE, meticillinresistant $S$. epidermidis; $\mathrm{EC}_{50}$, half-maximal effective concentration; $\mathrm{IC}_{50}$, half-maximal inhibitory concentration; MIC, minimum inhibitory concentration; Me, methyl.

${ }^{1}$ Corresponding author: phone: +7 (499) 246-69-83; e-mail: anton2rin@gmail.com. to combat antimicrobial resistance [4], the Strategy for preventing the spread of antimicrobial resistance in the Russian Federation [5].

According to the analysis of the introduction of low-molecular-weight compounds into medical practice in 1981-2019, natural substances and their semisynthetic derivatives and mimetics are an important source of new therapeutic agents. In some areas (for example, the use of antibacterial and anticancer agents), they are the main source of new medicines [6]. Thus, the search and study of compounds from various natural sources remains one of the key directions in the development of new anti-infectious agents.

However, the traditional (phenotypic) approach to screening and revealing active substances is unable to significantly expand the chemical range of secondary metabolites. One of the ways to solve this problem is to study hard-to-reach and little-explored habitats. Recently, there has been a sharp increase in the number of studies of secondary metabolites from extremophiles. Extremophilic microorganisms are capable of functioning normally at extremely high or low temperatures, pressure, and in other environmental con- 


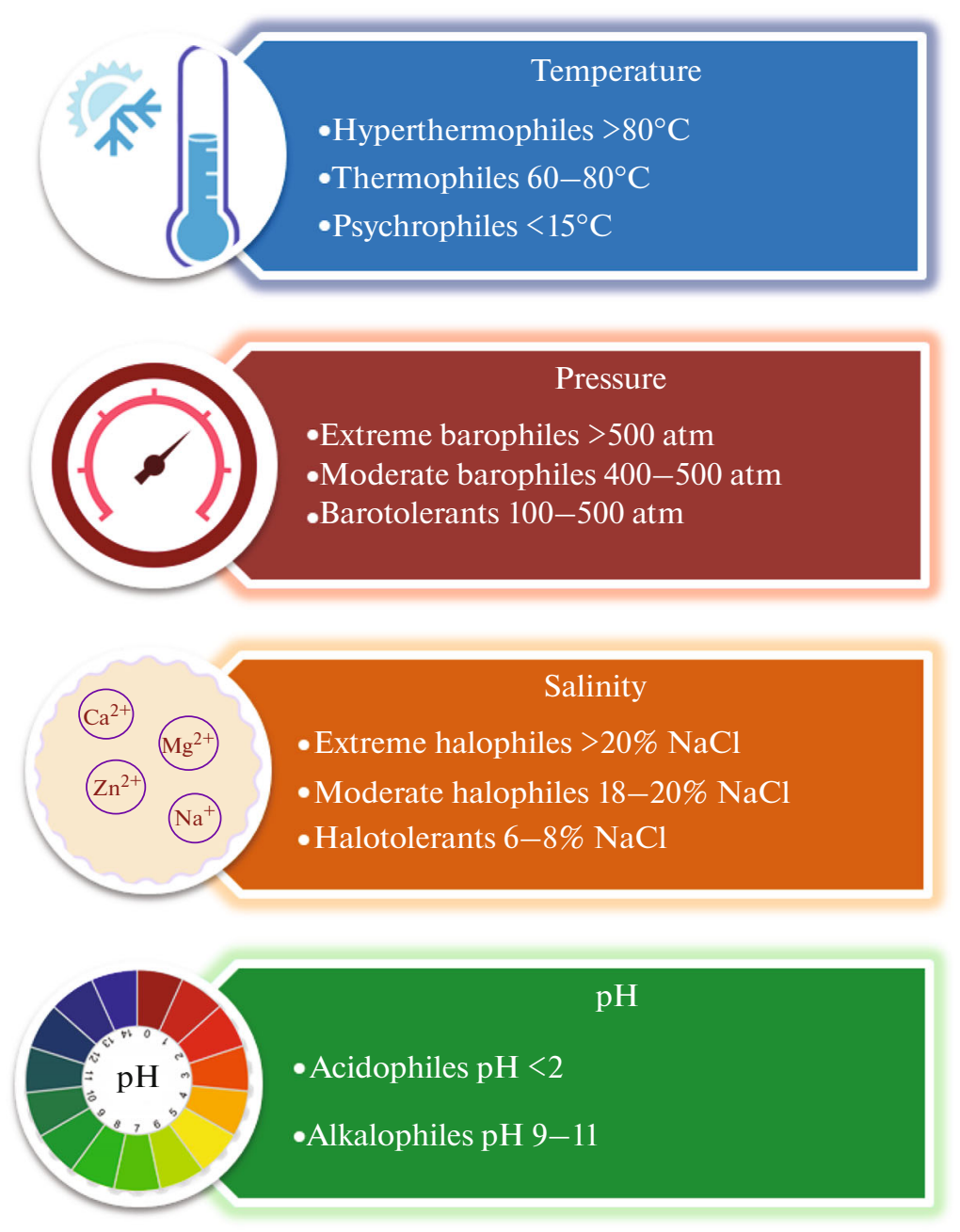

Fig. 1. Common adaptation factors for extremophiles.

ditions. For several decades, microbiologists have paid attention to these organisms because of the biotechnological potential of their enzymes (so-called extremozymes), which are used in the production of food and detergents and other industries. At the same time, the inhabitants of extreme econiches remained nearly unexplored for a long time in terms of the chemistry of natural compounds. Current trends in biotechnology indicate a high potential of the inhabitants of such econiche as producers of new specialized metabolites with a wide variety of properties [7]. As a rule, extremophiles are classified by a broad-spectrum of adaptation factors. For the producers considered in this paper, these factors are temperature, salinity, pressure, solar radiation, and $\mathrm{pH}$ level (Fig. 1). Organisms that can adapt to several factors are called polyextremophiles.

Fungi and fungi-like microorganisms are the most numerous eukaryotic organisms on the planet, and their presence in a wide variety of extreme ecosystems makes them important objects for researchers [8]. Since the discovery of penicillin to the present day, micromycetes, along with actinobacteria, are one of the most productive sources of antibiotic compounds for medicine and agriculture [9]. Many authors consider extremophilic micromycetes as a promising source of small molecules with an unusual mode of action or significant structural novelty. This work generalizes the latest literature data (2018-2019) on secondary metabolites of extremophilic micromycetes with antibiotic activity.

Natural compounds including bioactive ones, which were isolated from extremophilic fungi with various types of adaptation, were considered in a recent review of the literature data up to 2017 [10]. There are also several specialized reviews about the biotechnological potential of fungi and other microorganisms with different types of adaptation, i.e., psychrophiles [11, 12], acidophiles [13], and halophiles [14]. 
Several extreme habitats that combine distinct adaptation factors are emphasized in this review. First of all, these are micromycetes isolated from marine habitats at a depth of less than $200 \mathrm{~m}$ (from marine sponges, sediments of coastal zones except for the Arctic/Antarctic shelf, shellfish, corals, algae, ascidia, etc.), which are designated further by the descriptor "marine." Marine ecosystems are very diverse in terms of temperature fluctuations (from $-1.5^{\circ} \mathrm{C}$ in marine ice to $400^{\circ} \mathrm{C}$ in deep-sea hydrothermal vents), pressure (1-1000 atm), illumination (from total darkness to euphotic zones), and composition and concentration of nutrients [15]. Fungi often associate with various marine invertebrates, algae, and plants, which contribute to nutrient cycling and organic decomposition [16]. Due to the unique features of polyextremophilic adaptation and a wide range of secondary metabolites, marine fungi are an interesting object in terms of expanding the pharmacophore space [7, 17]. High interest in marine micromycetes is reflected in some reviews of the literature data on their biodiversity and the spectrum of metabolites [18-35]. Secondary metabolites of marine micromycetes are mentioned in reviews concerning the biotechnological potential of the entire biodiversity of marine microorganisms [18, $24,26,27,33,34]$. They are also described in several specialized reviews. In recent years, generalized data on active secondary metabolites of micromycete genera, which are common among marine microorganisms, i.e., Phoma [28], Penicillium [21], and Aspergillus [30] have been summarized. A growing number of publications on new biologically active compounds isolated from marine micromycetes have led to reviews of both their active metabolites in general [19, 20, 23, 29], and specific chemical classes of compounds, i.e., anthraquinones [22] and peptides [32].

The "deep-sea" descriptor refers to producers from marine habitats at a depth of $200 \mathrm{~m}$ or more (samples of sediments, water, etc.). The deep-sea environment is one of the most extensive ecosystems on the planet because $95 \%$ of the Earth's oceans are more than 1000 $m$ deep. These habitats are characterized by temperatures below $2^{\circ} \mathrm{C}$, complete darkness at depths of more than $250 \mathrm{~m}$, and low oxygen levels. Deep-sea fungi are mainly present in seafloor sediments $[15,16]$. Antibiotic secondary metabolites of fungi from deep-sea habitats are mentioned in several recent reviews on the biotechnological potential and biodiversity of deepsea econiche [26], anoxic water fungi [31], and other deep-sea habitats [35].

The "mangrove" descriptor refers to microorganisms isolated from mangrove soils and roots, branches, leaves, and fruits of mangrove plants. Over the past 15 years, the number of secondary metabolites from fungi of mangrove plants has been steadily increasing, which indicates the great potential of this ecological niche. Researchers have recently generalized the data on some bioactive compounds of mangrove soil fungi, endophytes of mangrove plants [27], and secondary metabolites of mangrove fungi of the Talaromyces genus [25].

The "Arctic/Antarctica" descriptor identified micromycetes isolated from seafloor sediments of coastal areas, soils, and soil-like formations of the Arctic and Antarctic zones.

Substances of different types of antibiotic (antiviral, antibacterial, antifungal, cytotoxic, and antihelminthic) activity are grouped by structure type taking into account their biosynthetic origin (peptides, diketopiperazines and related compounds, polyketides, xanthonoids and related compounds, alkaloids, terpenoids, steroids and related compounds, and compounds of mixed and unidentified origin). Polypeptide compounds, due to their large number, are divided into several groups by the type of chemical structure (quinones, chromones, benzophenones, xanthones, and other polyketides). The configurations of stereocenters are indicated for compounds, which have been studied in this regard in the original works. Planar structural formulas are given for compounds, the relative configuration of which has been determined by the NMR spectra. The structures and numbers of substances previously described and reidentified are grayed out in tables and figures.

\section{PEPTIDES, DIKETOPIPERAZINES, AND RELATED COMPOUNDS}

Among the biologically active metabolites of extremophilic micromycetes, some peptide compounds of various structural types were identified, i.e., a new family of small cyclic peptides (1-5), a series of diketopiperazines and related compounds (6-21), and two types of linear peptides with pronounced cytotoxicity $(\mathbf{2 2}-\mathbf{2 5}, \mathbf{2 6})$. Data on the biological activity of compounds are summarized in Table 1.

Cyclic peptides, so-called acremonpeptides A-D (1-4), isolated from marine micromycetes [36] are natural siderophores. The chelating structural fragment of acremonpeptide molecules is of a hydroxamate nature and formed by the $N^{5}$-hydroxy- $N^{5}$-acetylL-ornithine residues (Fig. 2). The high affinity of these compounds for trivalent metal ions causes acremonpeptide $\mathrm{D}$ to also be released as a complex with aluminum(III) ions (5). The moderate antiviral activity of acremonpeptides is of main interest.

In recent years, extremophilic micromycetes have become the source of some new compounds, which belong to the structural class of diketopiperazines. For example, a marine fungus of the Penicillium genus [37] has been the source for 2,5-diketopiperazine called penicillatide B (6), which belongs to the family of previously known peptides isolated from other marine sources [38, 39]. Recently, a new member of the fusaperazine family, fusaperazine F (9), with moderate cytotoxicity, was isolated from an extremophilic micromycete [40]. Interestingly, its well-known ana- 
Table 1. Data on the origin and biological activity of peptide antibiotics

\begin{tabular}{|c|c|c|c|c|c|}
\hline Cpd. & Producer/species & $\begin{array}{l}\text { Extremophile } \\
\text { type/origin }\end{array}$ & Activity/toxicity & $\mathrm{EC}_{50}, \mathrm{IC}_{50}, \mathrm{MIC}$ & Ref. \\
\hline $1-5$ & $\begin{array}{l}\text { Acremonium persicinum } \\
\text { SCSIO } 115\end{array}$ & Marine & AV & $\begin{array}{l}\mathbf{E C}_{\mathbf{5 0}} \mathbf{8 . 7 - 1 6} \boldsymbol{\mu} \mathbf{M} \\
\text { (Herpes Simplex Virus) }\end{array}$ & {$[36]$} \\
\hline 7 & Penicillium sp. & Marine & $\begin{array}{l}\mathrm{CT} \\
\mathrm{AB}\end{array}$ & $\begin{array}{l}\text { IC }_{\mathbf{5 0}} \mathbf{2 3 . 0}-\mathbf{5 0} \boldsymbol{\mu} \mathrm{M}(\mathrm{HCT}-116, \text { HepG } 2, \\
\mathrm{MCF}-7) \\
\mathbf{I C}_{\mathbf{5 0}} \mathbf{3 8 . 9 - 1 0 2} \boldsymbol{\mu M}(\mathrm{HCT}-116, \text { HepG } 2, \\
\mathrm{MCF}-7)\end{array}$ & [37] \\
\hline 8 & $\begin{array}{l}\text { Penicillium sp. } \\
\text { Penicillium sp. } \\
\text { SCSIO } 06720\end{array}$ & $\begin{array}{l}\text { Deep-sea } \\
\text { Marine } \\
4762 \mathrm{~m}\end{array}$ & $\begin{array}{l}\text { CT } \\
\mathrm{AB}\end{array}$ & $\begin{array}{l}\text { IC }_{\mathbf{5 0}} \mathbf{9 4 . 0}-\mathbf{1 1 4} \boldsymbol{\mu M}(\mathrm{HCT}-116, \text { HepG } 2, \\
\mathrm{MCF}-7) \\
\mathrm{MIC} 0.10-\mathbf{0 . 1 3} \boldsymbol{\mu g} / \mathbf{m L} \text { (Vibrio anguillarum) } \\
{[58]}\end{array}$ & $\begin{array}{l}{[37]} \\
{[59]}\end{array}$ \\
\hline 9 & $\begin{array}{l}\text { Penicillium crustosum } \\
\text { HDN } 153086\end{array}$ & Antarctica & CT & $\mathrm{IC}_{50} \mathbf{1 2 . 7 \mu \mathrm { M } ( \mathrm { K } 5 6 2 )}$ & [40] \\
\hline 10 & Aspergillus ochraceus & Marine & CT & $\mathrm{IC}_{\mathbf{5 0}} \mathbf{3 . 0} \boldsymbol{\mu M}(\mathrm{A} 2780)$ & [42] \\
\hline 11 & Aspergillus sydowii SP-1 & Antarctica & $\mathrm{AB}$ & $\begin{array}{l}\text { MIC 0.12-1.0 } \boldsymbol{\mu g} / \mathbf{m L} \text { (Staphylococcus } \\
\text { aureus, MRSA, S. epidermidis, MRSE) }\end{array}$ & [44] \\
\hline 12 & $\begin{array}{l}\text { Penicillium chrysogenum } \\
\text { MCCC 3A00292 }\end{array}$ & $\begin{array}{l}\text { Deep-sea } \\
2076 \mathrm{~m}\end{array}$ & $\mathrm{CT}$ & $\begin{array}{l}\text { IC }_{\mathbf{5 0}} \mathbf{7 . 8 1}-\mathbf{8 . 3 4} \boldsymbol{\mu M}(\mathrm{BEL}-7402, \mathrm{BIU}- \\
87 \mathrm{~K} 562)\end{array}$ & {$[46]$} \\
\hline 13 & Talaromyces purpurogenus & Marine & $\mathrm{CT}$ & $\mathrm{IC}_{50}$ 8.29-9.71 $\boldsymbol{\mu M}(\mathrm{A}-549, \mathrm{HL}-60)[55]$ & [54] \\
\hline $\begin{array}{l}16 \\
17\end{array}$ & $\begin{array}{l}\text { Penicillium granulatum } \\
\text { MCCC 3A } 00475\end{array}$ & $\begin{array}{l}\text { Deep-sea } \\
2284 \mathrm{~m}\end{array}$ & $\begin{array}{l}\mathrm{CT} \\
\mathrm{AB}\end{array}$ & $\begin{array}{l}\text { IC }_{\mathbf{5 0}} \mathbf{1 9 . 5} \boldsymbol{\mu M} \text { (HepG2) } \\
\text { Qualitative AB activity (Bacillus coagulans } \\
\text { IMM 311, B. licbeniformis IMM 308, } \\
\text { B. megateriurn DSM 32, B. subtilis IMM } \\
\text { 313, B. cereus IMM 307, B. stearotberrnopbi- } \\
\text { lus DSM 22, Mycobacterium phlei IMM 255, } \\
\text { S. aureus IMM 257, Micrococcus luteus } \\
\text { DSM 348, M. caseolyticus) [48] } \\
\text { IC }_{\mathbf{5 0}} \mathbf{1 3 - 3 3 . 6} \boldsymbol{\mu M} \text { (BEL-7402, A-549, } \\
\text { MOLT-4, HL-60) [49] }_{\text {IC }} \mathbf{5} \boldsymbol{\mu M} \text { (HepG2) }\end{array}$ & [47] \\
\hline 18 & Penicillium sp. TJ403-1 & Marine & $\mathrm{CT}$ & $\mathrm{IC}_{\mathbf{5 0}}$ 18.41-21.77 $\boldsymbol{\mu M}(\mathrm{A}-549, \mathrm{HL}-60)$ & {$[51]$} \\
\hline \multirow{2}{*}{$\begin{array}{l}20 \\
21\end{array}$} & \multirow{2}{*}{$\begin{array}{l}\text { Eurotium sp. } \\
\text { SCSIO F452 }\end{array}$} & \multirow[t]{2}{*}{ Marine } & \multirow[t]{2}{*}{ CT } & IC $_{\mathbf{5 0}}$ 12.5-15.0 $\boldsymbol{\mu M}(\mathrm{SF}-268, \mathrm{HepG} 2)$ & \multirow[t]{2}{*}{ [53] } \\
\hline & & & & IC $_{\mathbf{5 0}} \mathbf{3 0 . 1 - 3 7 . 3 \mu M}(\mathrm{HepG} 2, \mathrm{SF}-268)$ & \\
\hline $\begin{array}{l}22 \\
23 \\
24 \\
25\end{array}$ & Trichoderma velutinum & $\begin{array}{l}\text { At an altitude } \\
\text { of about } 800 \mathrm{~m} \\
\text { above sea level, } \\
\text { India/ } \\
\text { Psychrotolerant }\end{array}$ & $\mathrm{CT}$ & $\begin{array}{l}\text { IC }_{\mathbf{5 0}} \mathbf{3 0} \boldsymbol{\mu M}(\mathrm{A} 549) \\
\text { IC }_{\mathbf{5 0}} \mathbf{2 - 3 0} \boldsymbol{\mu M}(\mathrm{HL}-60, \mathrm{MDA}-\mathrm{MB}-231 \\
\mathrm{A} 549) \\
\text { IC }_{\mathbf{5 0}} \mathbf{1 6}-\mathbf{1 7} \boldsymbol{\mu} \mathbf{M}(\mathrm{HL}-60, \mathrm{MDA}-\mathrm{MB}-231 \\
\mathrm{A} 549) \\
\text { IC }_{\mathbf{5 0}} \mathbf{4 - 7} \boldsymbol{\mu M}(\mathrm{HL}-60, \mathrm{MDA}-\mathrm{MB}-231 \\
\text { A549, LS-180) }\end{array}$ & {$[56]$} \\
\hline 26 & Emericellopsis alkalina & $\begin{array}{l}\text { Lake Zhaltyr, } \\
\text { Kulunda steppe, } \\
\text { Russia/ } \\
\text { Haloalkalophilic }\end{array}$ & $\begin{array}{l}\mathrm{CT} \\
\mathrm{AB} \\
\mathrm{AF}\end{array}$ & $\begin{array}{l}\text { EC }_{\mathbf{5 0}} \mathbf{2 . 8} \boldsymbol{\mu M} \text { (HepG2) } \\
\text { MIC 4-32.5 } \boldsymbol{\mu g} / \mathbf{m L} \text { (Bacillus cereus ATCC } \\
\text { 14893, S. aureus FDA } 209 \text { P, Listeria mono- } \\
\text { cytogenes EGDe) } \\
\text { MIC } 2-\mathbf{4} \boldsymbol{\mu g} / \mathbf{m L} \text { (Candida sp., Aspergillus sp.) }\end{array}$ & {$[57]$} \\
\hline
\end{tabular}


$\log$, fusaperazin E [41], which differs in the geometry of the double bond, was not active. These compounds are typical representatives of the corresponding structural families.

Diketopiperazine waspergillamide B (10) that contains the unusual paranitrobenzoyl group has been obtained for the first time by culturing the marine Aspergillus ochraceus micromycete [42].

In addition to new compounds, some previously known active metabolites of the diketopiperazine class including cyclo-(D-Pro-D-Phe) (7) [38] and cyclo(D-Pro-L-Phe) (8) were discovered [39]. Their activity spectrum was refined and selective cytotoxicity was found against certain tumor cell lines. The antibacterial activity was also detected for the well-known diketopiperazine cyclo-(L-Trp-L-Phe) (11) [43], which was isolated from a psychrotolerant micromycete [44]. Cyclopenol (12), a known fungal metabolite [45] isolated from a seafloor fungus of the Penicillium chrysogenum species [46] showed moderate cytotoxic activity.

Representatives of the diketopiperazine class of alkaloid nature deserve a special mention. The chemical studies of secondary metabolites of the deep-sea seafloor Penicillium granulatum MC CC 3A00 475 micromycete [47] led to the discovery of a previously undescribed diketopiperazine, roquefortine $\mathbf{J}$ (14) along with three known analogs, i.e., the neurotoxins roquefortine C (15) [48], roquefortine $F(16)$ [49], and meleagrin (17) [50]. Weak cytotoxic activity was shown for well-known diketopiperazine alkaloid brevicompanine $G$ (18) [52] (structurally resembling the roquefortine family), which was isolated from the Penicillium sp. TJ403-1 coral fungus [51].

Three pairs of enantiomeric spirocyclic diketopiperazine alkaloids, called variecolortins A-C (19-21, Fig. 3) with an unusual spirocyclic scaffold were isolated from the Eurotium sp. SCSIO F452 marine micromycete [53].

Along with new terpenoids that showed noticeable activity, a diketopiperazine alkaloid 6-methoxyspirotrypostatin B (13) isolated from the culture fluid of the Talaromyces purpurogenus strain [54] previously showed the cytotoxic activity [55].

Peptaibols (nonribosomal linear oligopeptides with the reduced $\mathrm{C}$-terminal fragment that contain $\alpha$ branched amino acids, i.e., $\alpha$-aminoizobutanoic acid and/or isovaline) are a common type of peptide secondary metabolites of micromycetes among linear peptides. In recent years, two new representatives of this structural type, i.e., lipovelutibols A-D (22-25) [56] and emericellipsin A (26) [57] have been isolated from extremophilic micromycetes. Features of lipovelutibols B (23) and D (25) are the presence of nonproteinogenic amino acid D-isovaline and the absence of $\alpha$-aminoisobutanoic acid in their molecules, previously described among all peptaibols. What is especially interesting is that these compounds show a more pronounced cytotoxic activity than analogs (23) and (25) that contain $\alpha$-aminoisobutanoic acid (lipovultibols A and C). Another peptaibol isolated from an extremophilic micromycete, emericellipsin A (26), has a noticeable antifungal activity and pronounced cytotoxicity. An interesting feature of this compound is the ability to inhibit the formation of bacterial biofilms in the almost complete absence of antibacterial properties.

\section{POLYKETIDES, XANTHONOIDS AND RELATED COMPOUNDS}

\subsection{Quinones}

Polyketides, which can be assigned to quinones, were quite widely represented among the secondary metabolites of extremophilic micromycetes isolated during the period covered in this work. Various substituted anthraquinones $(\mathbf{2 7}-\mathbf{2 9}, \mathbf{3 0}-\mathbf{3 5}, \mathbf{3 8}-\mathbf{4 1})$, dimeric anthraquinones (42-44), a series of perylene quinones $(\mathbf{4 5}-\mathbf{5 0}, \mathbf{3 7})$, and tricyclic naphthoquinones (51-53) were isolated. Data on the activity of new and known quinones are summarized in Table 2.

Among the new and active anthraquinones, we should note similar compounds (38) and (39), the producer of which (Alternaria sp.) was identified as a result of the large-scale screening of strains of marine origin [60]. Despite the similarity of chemical structures, compound (38) showed approximately four times less antibacterial activity than compound (39). At the same time, both compounds showed a similar moderate level of antifungal activity. Another new anthraquinone (30) isolated from a deep-sea micromycete [61] showed a noticeable antibacterial activity. According to molecular docking, these compounds can inhibit topoisomerase IV and AmpC $\beta$-lactamase. An interesting example of expanding the chemical space of secondary metabolites and applying the OSMAC strategy (One Strain-Many Compounds [62]) is the production of new anthrachinone from isoversicolorin C (41). Cultivation of the Aspergillus nidulans MA143 mangrove fungus using ethanol stress (in the presence of $0.1 \%$ ethanol) [63] resulted in the production of compound (41) along with its close analog, versicolorin C pigment (40) [64].

Other isolated anthraquinones were previously described compounds, for some of which the biological activity was detected or clarified. For example, in addition to the new anthraquinone (30), damnacanthal (31) [65], xanthopurpurin (32) [66], 6-hydroxyrubiadin (33) [67], and emodin (34) [68-72] were isolated from the Aspergillus versicolor seafloor micromycete [61]. Emodin (34) was also isolated from a variety of other extremophilic micromycetes, i.e., thermo- 


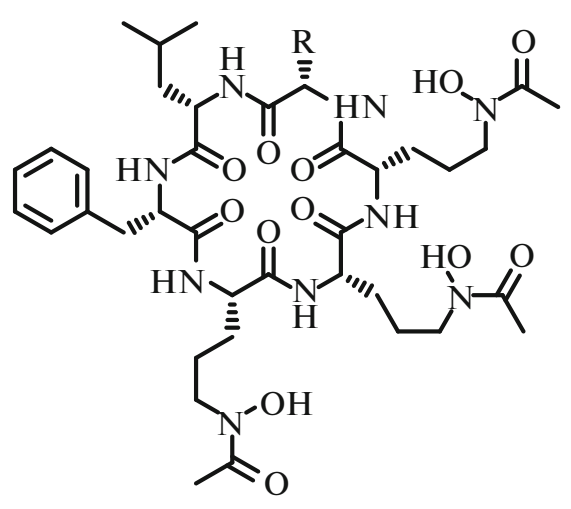<smiles>[Te]=Cc1ccccc1</smiles>

(1) $\mathrm{R}=\mathrm{CH}_{2} \mathrm{OH}$

(2) $\mathrm{R}=\mathrm{Me}$

(3) $\mathrm{R}=\mathrm{A}$

(4) $R=B$<smiles>CS[C@H]1C(=O)N(C)C(=Cc2ccc(OCC=C(C)C)cc2)C(=O)N1C</smiles>

Fusaperazine F

(9)<smiles>CC(C)C[C@@]1(O)NC(=O)C(C(C)(C)OC(=O)CNC(=O)c2ccc([N+](=O)[O-])cc2)NC1=O</smiles>

Waspergillamide B

(10)

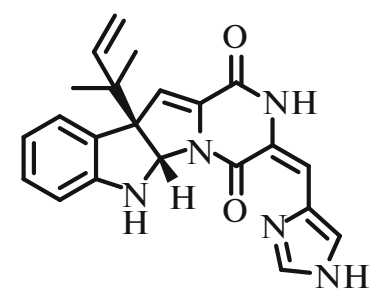

Roquefortine $\mathrm{J}$

(14)

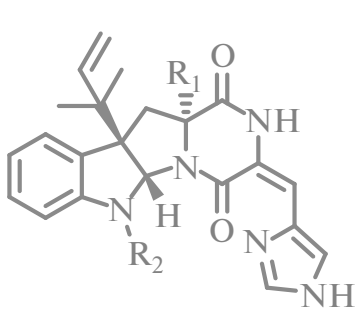

Roquefortine $\mathrm{C}$ (15) $\mathrm{R}_{1}=\mathrm{H}, \mathrm{R}_{2}=\mathrm{H}$

Roquefortine $\mathrm{F}$

(16) $\mathrm{R}_{1}=\mathrm{H}, \mathrm{R}_{2}=\mathrm{OCH}_{3}$

(11)<smiles>C=C1N[C@H](Cc2ccccc2)C(=O)N2CCC[C@H]12</smiles>

Penicillatide B

(6)

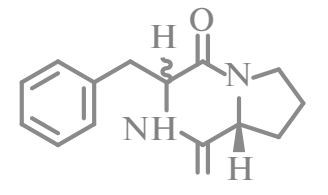

Cyclo-(D-Pro-L-Phe)

Cyclo-(D-Pro-D-Phe)

(8)

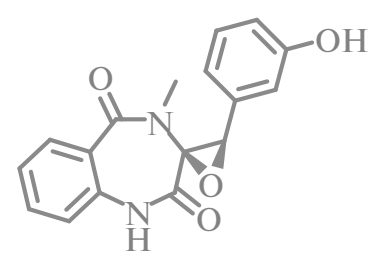

Cyclopenol

(12)

6-Methoxyspirotryprostatin B

(13)

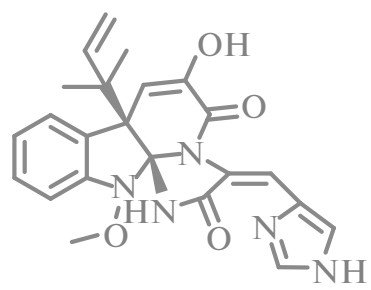

Meleagrin

(17)

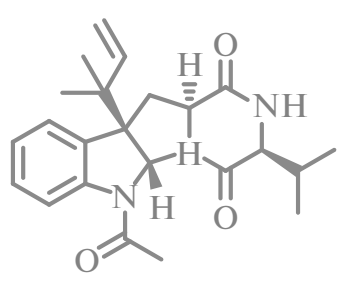

Brevicompanine $\mathrm{G}$

(18)

Fig. 2. Cyclic peptides and diketopiperazines. 


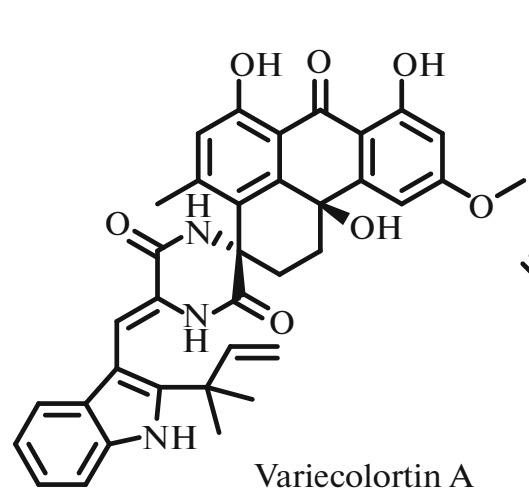

(19)

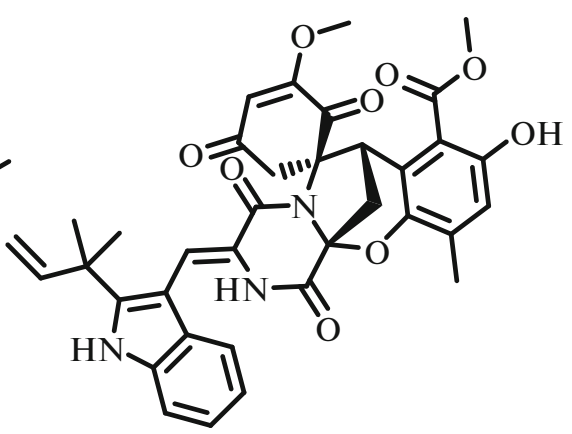

Variecolortin B

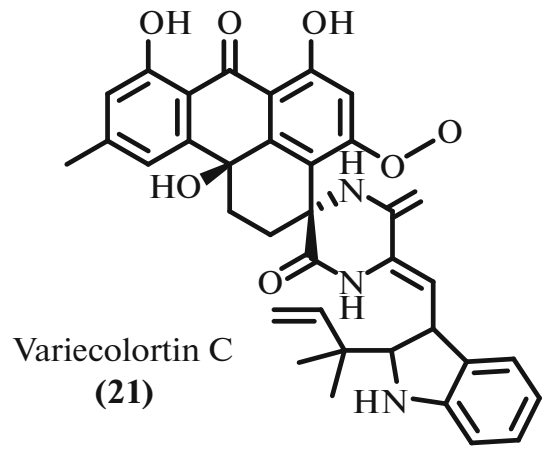

(20)

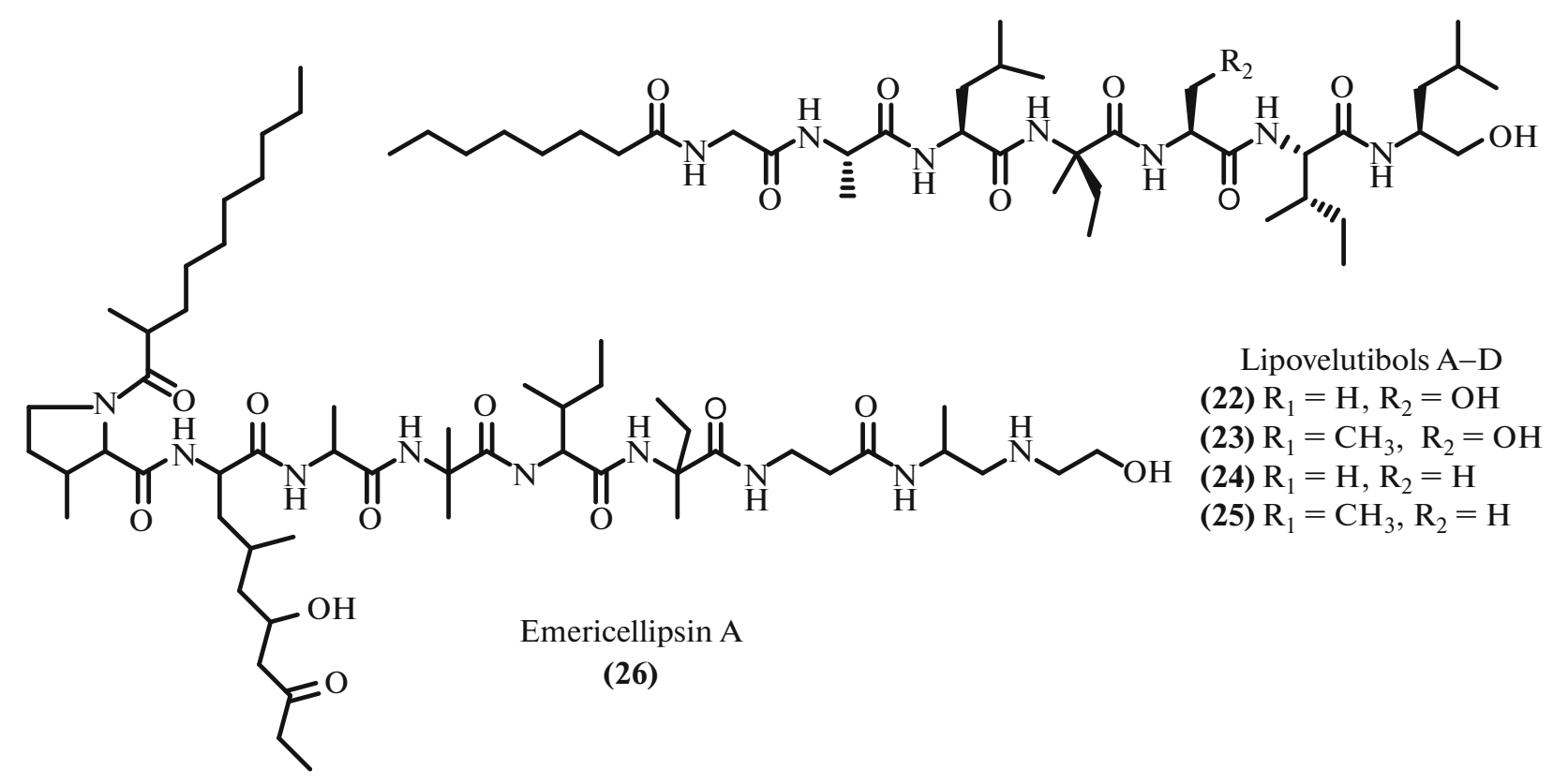

Fig. 3. Linear peptides and mixed diketopiperazines.

philic Penicillium sp. RO-11 [73], marine fungi of the Aspergillus genus [74, 75], marine Cochliobolus lunatus SCSIO41401 [76], deep-sea Aspergillus sydowii C1S01-A7 micromycete [77], marine Penicillium sp. ZZ380 [78], and Aspergillus candidus KUFA0062 [79]. In some cases, new cytotoxic [76] and antitubercular [75] types of bioactivity were revealed for emodin (34). Known emodin-related anthraquinones, i.e., questin (34) [70], physcion (35) [80], and chrysophanic acid (chrysophanol) (36) [79, 81, 82] turned out to be significantly less common.

Dimeric anthraquinones (42) and (43) that contain the unusual $\mathrm{C}-\mathrm{O}-\mathrm{C}$ ether bond have been isolated from the culture fluid of the Aspergillus versicolor marine strain [83]. These new biologically active compounds showed selective antibacterial activity against Staphylococcus aureus. The well-known anthraqui- nones methylaverantin (27) [84], averantin (28) [84], and averytrin (29) [85] were also isolated. Another example of the dimeric anthraquinone is a well-known mycotoxin, viomellein (44) [86]. The source of this compound was micromycetes of the Aspergillus genus [42] isolated from a sea sponge. Viomellein showed high cytotoxicity against the human ovarian carcinoma A2780 and the mouse lymphoma L5178Y cell lines.

Study of the marine strains of the Alternaria genus (Alternaria sp. SCSIO41014 [87], Alternaria sp. [60], and Alternaria alternata L3111' [88]) led to the identification of some representatives of the structural family of perylene quinones. In particular, two new perylene quinones, i.e., altertoxin VII (45) and a new derivative (46) were isolated [87] along with active structural analogs, i.e., altertoxin I (47) [89], stem- 
Table 2. Data on the origin and biological activity of quinones

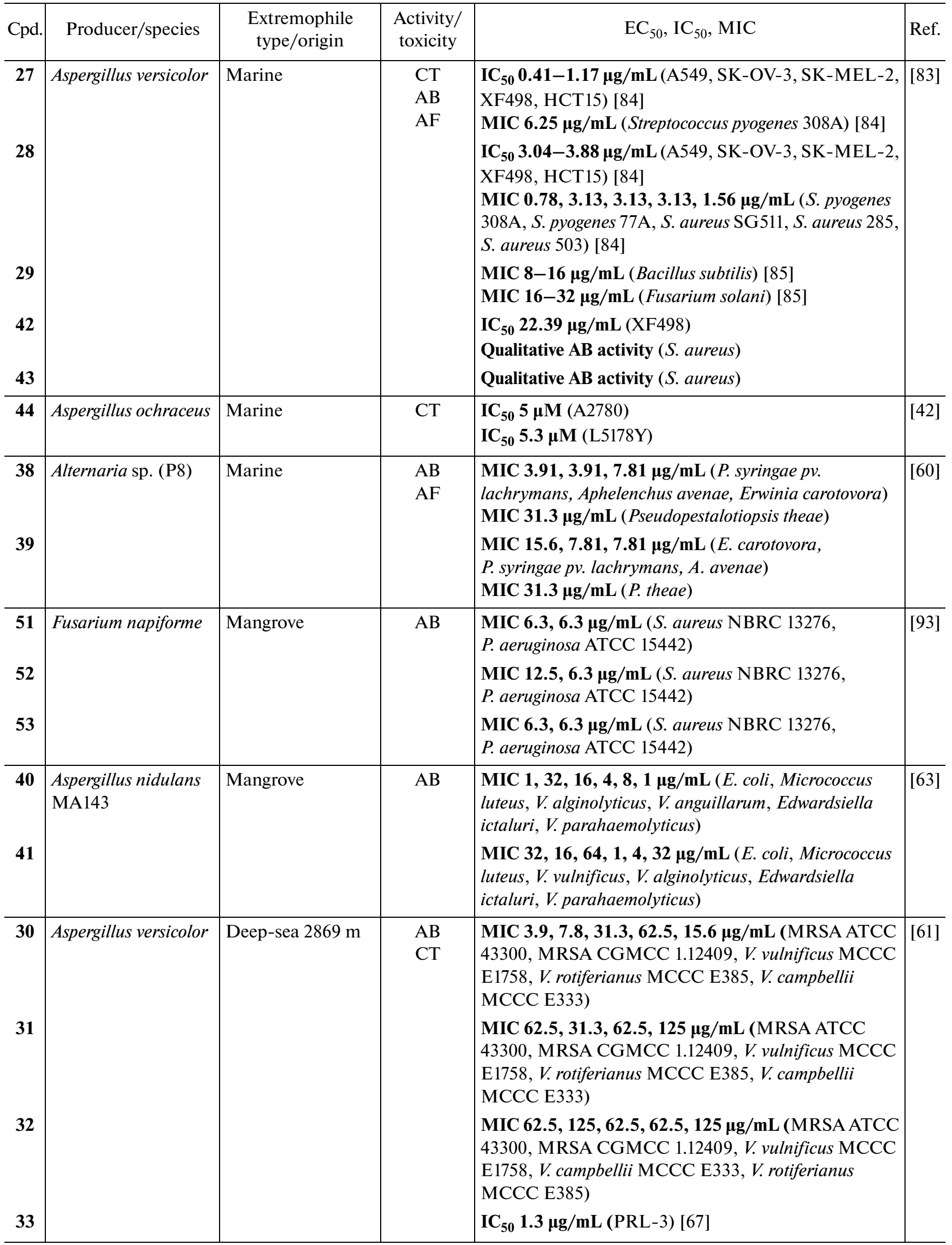


Table 2. (Contd.)

\begin{tabular}{|c|c|c|c|c|c|}
\hline Cpd. & Producer/species & $\begin{array}{l}\text { Extremophile } \\
\text { type/origin }\end{array}$ & $\begin{array}{l}\text { Activity/ } \\
\text { toxicity }\end{array}$ & $\mathrm{EC}_{50}, \mathrm{IC}_{50}, \mathrm{MIC}$ & Ref. \\
\hline 34 & $\begin{array}{l}\text { Aspergillus versicolor } \\
\text { Penicillium sp. } \\
\text { Cochliobolus lunatus } \\
\text { Aspergillus fumigatus } \\
\text { Aspergillus sydowii } \\
\text { Penicillium sp. } \\
\text { Aspergillus candidus } \\
\text { Aspergillus sp. }\end{array}$ & $\begin{array}{l}\text { Deep-sea } 2869 \mathrm{~m} \\
\text { Hot spring } \\
\text { deposits }\left(45-65^{\circ} \mathrm{C}\right) \text {, } \\
\text { Saudi Arabia/ } \\
\text { Thermophile } \\
\text { Marine } \\
\text { Marine } \\
\text { Deep-sea } 4950 \mathrm{~m} \\
\text { Marine } \\
\text { Marine } \\
\text { Marine }\end{array}$ & $\begin{array}{l}\mathrm{AT} \\
\mathrm{AB} \\
\mathrm{CT}\end{array}$ & 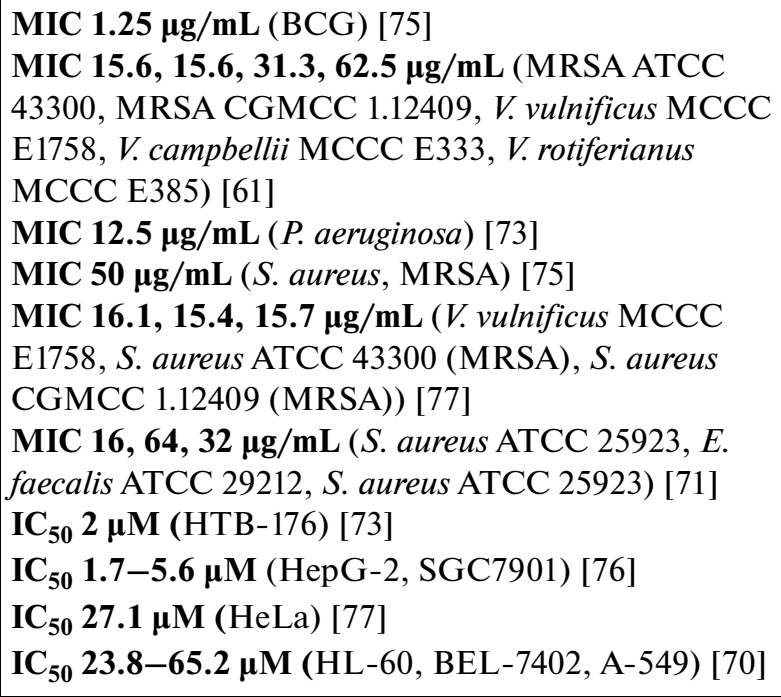 & \begin{tabular}{|l}
{$[61]$} \\
{$[73]$} \\
{$[76]$} \\
{$[75]$} \\
{$[77]$} \\
{$[78]$} \\
{$[79]$} \\
{$[74]$}
\end{tabular} \\
\hline 35 & Aspergillus sp. & Marine & $\mathrm{CT}$ & $\mathrm{IC}_{50} 73.2 \mu \mathrm{M}(\mathrm{HL}-60)[70]$ & [74] \\
\hline 36 & Penicillium sp. ZZ901 & Marine & $\begin{array}{l}\mathrm{CT} \\
\mathrm{AB}\end{array}$ & $\begin{array}{l}\mathrm{IC}_{50} 34.68 \mu \mathrm{M}(\mathrm{U} 87 \mathrm{MG} \text { glioma cells) } \\
\mathrm{IC}_{50} 30.22 \mu \mathrm{M}(\mathrm{C} 6)\end{array}$ & [94] \\
\hline 48 & $\begin{array}{l}\text { Alternaria } \text { sp. } \\
\text { SCSIO41014 }\end{array}$ & Marine & $\mathrm{CT}$ & 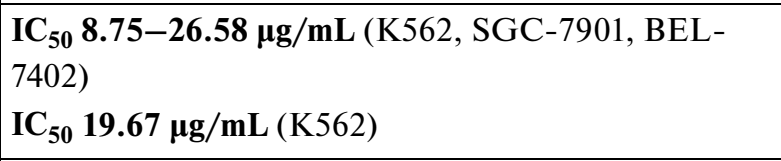 & [87] \\
\hline 49 & $\begin{array}{l}\text { Alternaria sp. } \\
\text { Alternaria alternata } \\
\text { L3111' }\end{array}$ & Marine & $\begin{array}{l}\mathrm{AB} \\
\mathrm{CT}\end{array}$ & $\begin{array}{l}\text { MIC } 1.95 \mu \mathrm{g} / \mathrm{mL} \text { (Clavibacter michiganensis) [60] } \\
\text { IC }_{\mathbf{5 0}} \mathbf{2 . 4 - 3 . 1} \boldsymbol{\mu g} / \mathbf{m L} \text { (A-549, HCT-116, HeLa) [88] }\end{array}$ & $\begin{array}{l}{[60]} \\
{[88]}\end{array}$ \\
\hline 47 & $\begin{array}{l}\text { Alternaria sp. } \\
\text { SCSIO41014 } \\
\text { Alternaria alternata } \\
\text { L3111' }\end{array}$ & Marine & AV & $\mathrm{IC}_{50} 1.42-2.2 \mu \mathrm{M}(\mathrm{HIV}-1)$ [95] & $\begin{array}{l}{[87]} \\
{[88]}\end{array}$ \\
\hline 50 & $\begin{array}{l}\text { Alternaria } \text { sp. } \\
\text { SCSIO41014 } \\
\text { Alternaria } \text { sp. } \\
\text { Alternaria alternata } \\
\text { L3111' }\end{array}$ & Marine & $\mathrm{AF}$ & $\begin{array}{l}\text { MIC } 7.81 \mu \mathrm{g} / \mathrm{mL} \text { (P. theae) [60] } \\
\text { MIC } 125 \mu \mathrm{g} / \mathrm{mL} \text { (A. brassicicola) [60] }\end{array}$ & $\begin{array}{l}{[87]} \\
{[60]} \\
{[88]}\end{array}$ \\
\hline 37 & $\begin{array}{l}\text { Aspergillus candidus } \\
\text { KUFA0062 }\end{array}$ & Marine & $\mathrm{AV}$ & $\begin{array}{l}\mathbf{E C}_{\mathbf{5 0}} \mathbf{0 . 2 1}, \mathbf{0 . 0 2} \boldsymbol{\mu g} / \mathbf{m L} \text { (Poliovirus type 2, Poliovirus } \\
\text { type 3, Coxsackievirus type A21, Coxsackievirus type } \\
\text { B4, Human rhinovirus type 2, Ross River virus, Her- } \\
\text { pes simplex virus type 1) [82] }\end{array}$ & [79] \\
\hline
\end{tabular}

phyperylenol (50) [60], 6-epi-stemphytriol (48) [90], and alterperylenol (49) [91]. The biological properties of known compounds have been clarified. For example, only alterperylenol (49) but not its analogs (47) and (50) showed cytotoxic properties [88]. The cytotoxicity tests [87] showed a noticeable and selective activity of the previously described compound 6-epi- stemphytriol (48), which makes it promising for further study as an antitumor agent.

Another structural family of secondary metabolites of extreme micromycetes turned out to be the naphthoquinone derivatives. The new derivatives, 6-hydroxy-astropaquinone $\mathrm{B}$ (51) and astropaquinone $\mathrm{D}$ (52), and well-known 3-O-methyl-9-Omethylfusarubin (53) [92] were isolated from the cul- 


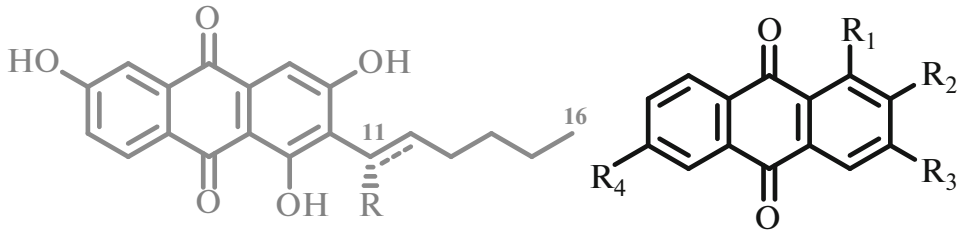

Methylaverantin (27) $\mathrm{R}=\mathrm{OCH}_{3}$

Averantin

(28) $\mathrm{R}=\mathrm{OH}$

Averythrin

(29) $\mathrm{R}=\mathrm{H}, \Delta^{11-12}$
(30) $\mathrm{R}_{1}=\mathrm{OH}, \mathrm{R}_{2}=\mathrm{CH}\left(\mathrm{OCH}_{3}\right)_{2}, \mathrm{R}_{3}=\mathrm{H}, \mathrm{R}_{4}=\mathrm{H}$

$$
\text { Damnacanthal }
$$

(31) $\mathrm{R}_{1}=\mathrm{OCH}_{3}, \mathrm{R}_{2}=\mathrm{CHO}, \mathrm{R}_{3}=\mathrm{OH}, \mathrm{R}_{4}=\mathrm{H}$

Xanthopurpurin

(32) $\mathrm{R}_{1}=\mathrm{OH}, \mathrm{R}_{2}=\mathrm{H}, \mathrm{R}_{3}=\mathrm{OH}, \mathrm{R}_{4}=\mathrm{H}$

6-Hydroxyrubiadin

(33) $\mathrm{R}_{1}=\mathrm{OH}, \mathrm{R}_{2}=\mathrm{CH}_{3}, \mathrm{R}_{3}=\mathrm{OH}, \mathrm{R}_{4}=\mathrm{H}$

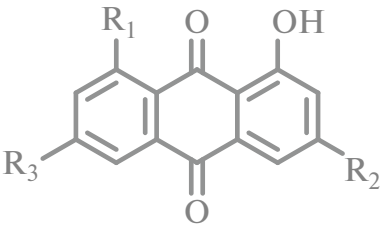

Emodin

(34) $\mathrm{R}_{1}=\mathrm{OH}, \mathrm{R}_{2}=\mathrm{OH}, \mathrm{R}_{3}=\mathrm{CH}_{3}$

Questin

(35) $\mathrm{R}_{1}=\mathrm{OMe}, \mathrm{R}_{2}=\mathrm{CH}_{3}, \mathrm{R}_{3}=\mathrm{OH}$

Physcion

(36) $\mathrm{R}_{1}=\mathrm{OH}, \mathrm{R}_{2}=\mathrm{OCH}_{3}, \mathrm{R}_{3}=\mathrm{CH}_{3}$

Chrysophanic acid

(37) $\mathrm{R}_{1}=\mathrm{OH}, \mathrm{R}_{2}=\mathrm{CH}_{3}, \mathrm{R}_{3}=\mathrm{H}$<smiles>CC(=O)c1cc2c(cc1O)C(=O)c1cc(O)cc(O)c1C2=O</smiles>

(38)<smiles>C[C@H](O)c1cc2c(cc1O)C(=O)c1cc(O)cc(O)c1C2=O</smiles><smiles>C[C@H]1c2cc3c(c(O)c2[C@H]2CC[C@H]1O2)C(=O)c1c(O)cc(O)cc1C3=O</smiles>

Versicolorin C

(40)<smiles>O=C1c2cc(O)cc(O)c2C(=O)c2c1cc(O)c1c2[C@H]2CCO[C@H]12</smiles>

Isoversicolorin $\mathrm{C}$

(41)

(39)<smiles>[R]OC(CCCCC)c1c(O)cc2c(c1O)C(=O)c1c(O)cc(Oc3cc(O)c4c(c3)C(=O)c3c(cc(O)c(C([R20])CCCCC)c3O)C4=O)cc1C2=O</smiles><smiles>COC1=C(c2c(OC)cc3cc4c(c(O)c3c2O)C(=O)O[C@H](C)C4)C(=O)c2c(cc3c(c2O)C(=O)O[C@@H](C)C3)C1=O</smiles>

(44)

Fig. 4. Anthraquinones and dimeric quinones.

ture fluid of the mangrove Fusarium napiforme endophyte [93]. The isolated compounds showed a noticeable phytotoxic effect in addition to moderate antibacterial activity.

\subsection{Chromones, Benzophenones, and Xanthones}

Various aromatic compounds were found among the secondary metabolites of extremophilic micromycetes, i.e., xanthones (54-68) and dihydroxanthenones (69-71), chromones and dihydrochromones (72-84), dimeric chromones (85-89), benzoquinones (90-97), and related compounds (98-110). Data on the origin and activity of aromatic polyamides are summarized in Table 3.
Xanthones are common antibiotic secondary metabolites of micromycetes. Several compounds of this class have been isolated from various extremophilic sources (Fig. 6).

Three new antibacterial prenylxanthones, aspergixanthones I-K (58-60), and four known analogs, i.e., aspergixanthone A (57) [97], 15-acetyl thajixanthone hydrate (61), thajixanthone hydrate (62) [98], and 16-chlorthajixanthone (63) [98, 99] were isolated from the marine fungus of the Aspergillus genus [96]. New aspergixanthone I (58) showed the greatest activity against the studied bacteria. Another new secondary metabolite of this class was 3-hydroxy pinselin (64) [100], which did not show antibiotic 
<smiles>O=C1CCC2=C3CC[C@@H](O)c4cc(O)cc(c43)C2=CC=C1O</smiles>

Altertoxin VII

(45)<smiles>COc1cc(OC)c2c(c1O)C(=O)C1=C(C[C@H](C)O[C@H]1OC)C2=O</smiles><smiles>CCCCOC(=O)C1(O)CC2=C3C=C[C@@H](O)Cc4ccc(O)c(c43)C2=CC=C1O</smiles>

(46)

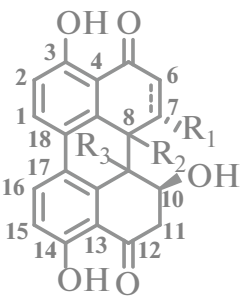

Altertoxin I

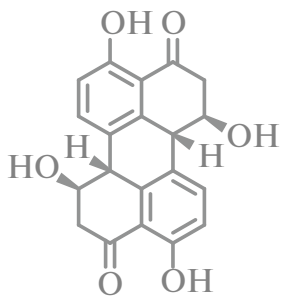

Stemphyperylenol

6-Epi-stemphytriol

(48) $8(S), 9(R) \mathrm{R}_{1}=\mathrm{OH}, \mathrm{R}_{2}=\mathrm{H}, \mathrm{R}_{3}=\mathrm{OH}$

Alterperylenol
(49) $8(R), 9(S) \mathrm{R}_{1}=\mathrm{H}, \Delta^{6-7}, \mathrm{R}_{2}=\mathrm{OH}, \mathrm{R}_{3}=\mathrm{H}$

6-Hydroxy-astropaquinone $\mathrm{B}$

(51) $\mathrm{R}=\mathrm{OCH}_{3}$

Astropaquinone $\mathrm{O}$

(52) $\mathrm{R}=\mathrm{H}$

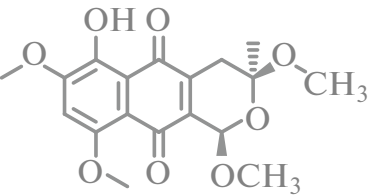

3-O-Methyl-9-O-methylfusarubin

(53)

Fig. 5. Perylenequinones and naphtoquionones.

activity. Some extremophilic micromycetes have become sources of known xanthones. 3,8-Dihydroxy-4-(2,3-dihydroxy-1-hydroxymethylpropyl)1-methoxyxanthone (55) [101] was isolated from seafloor fungus Penicillium chrysogenum MCCC 3A00292 [46]. The primary study of metabolites of a fungus of the Pseudopestalotiopsis genus [102] led to the isolation of the previously described dehydrate chloroisosulochrin (65) [103]. The well-known xanthone, cytotoxic stergmatocystin (54) [84], was obtained from the Aspergillus versicolor marine strain [83].

A deep-sea micromycete, Aspergillus sydowii C1S01-A7 [77], is a producer of two new compounds, 2-hydroxy-6-formyl-vertixanthone (66) and 12- $O$ acetyl-sydovinin A (67), and 22 known compounds including those with antibiotic activity, i.e., xanthones (sydovinin B (68) [104] and yikathin C (56) [105]) and their dihydro derivatives (aspergillusone A (69) [106] and AGI-B4 (70) [107]). A similar spectrum of metabolites was found in the marine fungus of the Aspergillus genus [100], i.e., 12-O-acetyl-AGI-B4 (71) [77] and aspergillusone A (69) [106]. AGI-B4 (70) showed noticeable cytotoxicity and selectivity compared to its structural (e.g., $O$-acylated) analogs. Thus, the dihydroxyxanthenone core and the free hydroxyl group at the $\mathrm{C}-12$ position are mandatory structural motifs for ensuring the cytotoxic activity of xanthones of this type (Fig. 6).
In addition to xanthones, chromone core-containing metabolites including previously undescribed compounds were isolated. New coniochaetone M (73) [108] along with the well-known analog, coniochaetone B (72) [78], which showed a low activity [109, 110] were isolated from a marine-derived fungus. Seafloor micromycete [59] was the source of one new and several known chromones, i.e., coniochaetone L (74), oxalicumone A (80) [111], $\beta$-diversonolic ester (81), and penicillocitrin A (82) [112].

Among three new polyketides, (2S)-2,3-dihydro5,6-dihydroxy-2-methyl-4H-1-benzopyran-4-one (75) with antibacterial properties was isolated from the $\mathrm{Col}$ letotrichum gloeosporioides mangrove endophyte [113]. Another new chromone, pseudopestalon (98), was obtained in the initial study of the abovementioned fungus of the Pseudopestalotiopsis genus [102]. The marine fungus of the Penicillium genus was a source of new chromones, i.e., pyanochromone (76), spirofuranochromone (77), and 7-hydroxy-6-methoxy-4oxo-3-[(1E)-3-oxobut-1-en-1-yl]-4H-chromene-5carboxylic acid (83) [114], along with known anhydrofulvic acid (84) [115].

We should also note the mangrove endophyte Cladosporium sp. [116], the source of a new glycosylated chromone (78) and the previously known 7-O$\alpha$-D-ribosyl-5-hydroxy-2-propyl-chromone [117], which showed cytotoxic properties.

Another structural family of aromatic polyketides isolated from extremophilic fungi is dimeric chro- 
Table 3. Data on the activity and origin of chromons, benzophenones, and xanthones

\begin{tabular}{|c|c|c|c|c|c|}
\hline Cpd. & Producer/species & $\begin{array}{l}\text { Extremophile } \\
\text { type/origin }\end{array}$ & $\begin{array}{l}\text { Activity/ } \\
\text { toxicity }\end{array}$ & $\mathrm{EC}_{50}, \mathrm{IC}_{50}, \mathrm{MIC}$ & Ref. \\
\hline 54 & Aspergillus versicolor & Marine & CT & $\begin{array}{l}\text { IC }_{\mathbf{5 0}} \mathbf{1 1 . 2 5 - 1 7 . 3 6} \boldsymbol{\mu g} / \mathbf{m L}(\mathrm{A}-549, \mathrm{SK}-\mathrm{OV}-3, \\
\mathrm{SK}-\mathrm{MEL}-2, \mathrm{XF} 498) \\
\text { IC }_{\mathbf{5 0}} \mathbf{1 . 8 6}-\mathbf{4 . 6 1} \boldsymbol{\mu} \mathbf{g} / \mathbf{m L}(\mathrm{A}-549, \mathrm{SK}-\mathrm{OV}-3 \\
\text { SK-MEL-2, XF498, HCT15) [84] }\end{array}$ & [83] \\
\hline $\begin{array}{l}69 \\
71\end{array}$ & $\begin{array}{l}\text { Aspergillus versicolor } \\
\text { MF160003 }\end{array}$ & Marine & AT & $\begin{array}{l}\text { MIC } 20 \mu \mathrm{g} / \mathrm{mL}(\mathrm{BCG}) \\
\text { MIC } 40 \mu \mathrm{g} / \mathrm{mL}(\mathrm{BCG})\end{array}$ & [100] \\
\hline 85 & Penicillium oxalicum & Marine & $\begin{array}{l}\mathrm{CT} \\
\mathrm{AB} \\
\mathrm{AF}\end{array}$ & $\begin{array}{l}\text { IC }_{\mathbf{5 0}} \mathbf{0 . 4 8 4 - 2 6 . 6} \boldsymbol{\mu} \text { M (BGC-823, SGC-7901, } \\
\text { HGC-27, EC9706, KYSE450, CNE1, CNE2, } \\
\text { SW620, SW480, LOVO, HuH-7, PLC/PRF/5, } \\
\text { SKHEP, A549, SKMES-1, SPC-A1, 95D, } \\
\text { Jeko-1, Raji, U937, A375, HFF, H22) } \\
\text { MIC 3.1 } \boldsymbol{\mu g} / \mathbf{m L} \text { (Sarcina lutea ATCC 9341) [119] } \\
\text { MIC } 12.5 \mu \mathrm{g} / \mathbf{m L} \text { (Bacillus subtilis CPI 219) [119] } \\
\text { MIC 6.3 } \boldsymbol{\mu g} / \mathbf{m L} \text { (Corynebacterium diphtheria } \\
\text { P.W.8) [119] } \\
\text { MIC 12.5 } \boldsymbol{\mu g} / \mathbf{m L} \text { (Micrococcus flavus ATCC } \\
\text { 10240) [119] } \\
\text { MIC 1.6 } \boldsymbol{\mu g} / \mathbf{m L} \text { (Trichophyton metagrophytes } \\
\text { IAM 8051) [119] }\end{array}$ & [118] \\
\hline 88 & $\begin{array}{l}\text { Penicillium oxalicum } \\
\text { Cladosporium } \mathrm{sp} . \\
\text { JS1-2 }\end{array}$ & $\begin{array}{l}\text { Marine } \\
\text { Mangrove }\end{array}$ & CT & $\begin{array}{l}\text { IC }_{\mathbf{5 0}} \mathbf{0 . 8 3 3 - 2 4 . 1} \boldsymbol{\mu M}(\mathrm{BGC}-823, \text { SGC-7901, } \\
\text { HGC-27, EC9706, KYSE450, CNE1, CNE2, } \\
\text { SW620, SW480, LOVO, HuH-7, PLC/PRF/5, } \\
\text { SKHEP, A549, SKMES-1, SPC-A1, 95D, } \\
\text { Jeko-1, Raji, U937, A375, HFF, H22) }\end{array}$ & $\begin{array}{l}{[118]} \\
{[121]}\end{array}$ \\
\hline 102 & $\begin{array}{l}\text { Penicillium erubes- } \\
\text { cens KUFA } 0220\end{array}$ & Marine & $\begin{array}{l}\mathrm{AB} \\
\mathrm{AF} \\
\mathrm{CT}\end{array}$ & 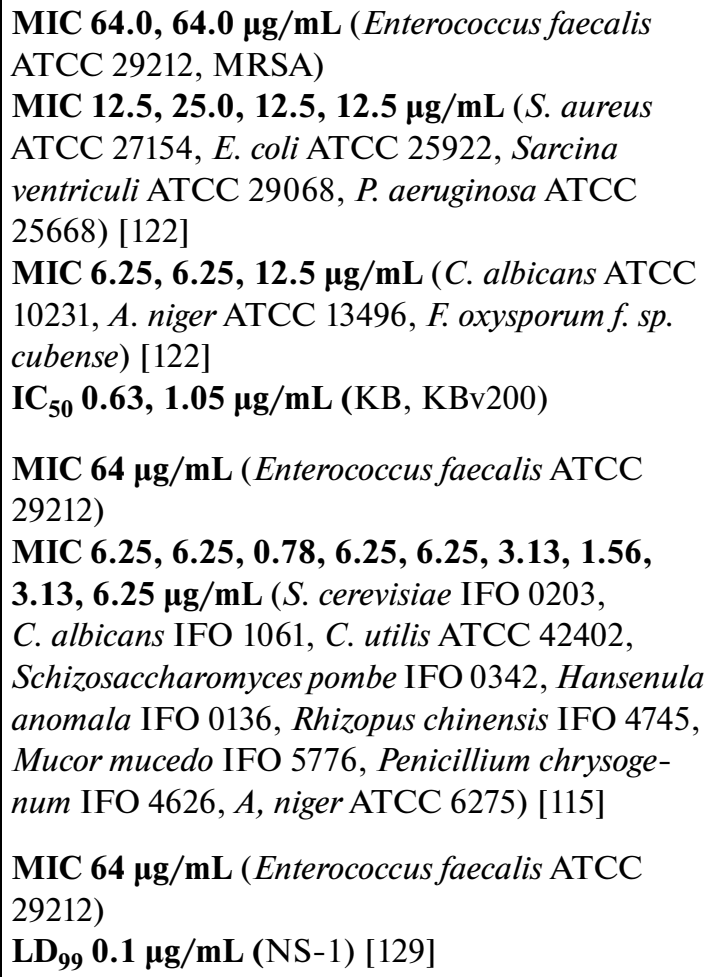 & [114] \\
\hline
\end{tabular}


Table 3. (Contd.)

\begin{tabular}{|c|c|c|c|c|c|}
\hline Cpd. & Producer/species & $\begin{array}{l}\text { Extremophile } \\
\text { type/origin }\end{array}$ & $\begin{array}{l}\text { Activity/ } \\
\text { toxicity }\end{array}$ & $\mathrm{EC}_{50}, \mathrm{IC}_{50}, \mathrm{MIC}$ & Ref. \\
\hline 90 & $\begin{array}{l}\text { Aspergillus sp. } \\
\text { Penicillium citrinum } \\
\text { HL-5126 }\end{array}$ & $\begin{array}{l}\text { Marine } \\
\text { Mangrove }\end{array}$ & $\begin{array}{l}\mathrm{AB} \\
\mathrm{CT} \\
\mathrm{AF}\end{array}$ & $\begin{array}{l}\text { MIC 7.53, } \mathbf{1 5 . 0 6 ,} \mathbf{3 0 . 1 2} \boldsymbol{\mu M} \text { (P. aeruginosa, } \\
\text { V. anguillarum, Aeromonas salmonicida) } \\
\text { IC }_{\mathbf{5 0}} \mathbf{6 3 . 4 , 8 3 . 1} \boldsymbol{\mu M}(\mathrm{HL}-60, \text { BEL-7402) [70] } \\
\text { MIC }>\mathbf{5 0} \boldsymbol{\mu g} / \mathbf{m L}(\text { T. metagrophytes IFO-5811, } \\
\text { Candida albicans IAM-4966, Pyricularia orysae } \\
\text { IFO-5279, A. fumigatus IAM-2004, } \\
\text { Helminthosporium sesamum IAM-5012) [125] }\end{array}$ & $\begin{array}{r}{[74]} \\
{[124]}\end{array}$ \\
\hline 65 & $\begin{array}{l}\text { Pseudopestalotiopsis } \\
\text { sp. PSU-AMF45 }\end{array}$ & Marine & $\mathrm{AF}$ & $\begin{array}{l}\text { MIC } 200 \mu \mathrm{g} / \mathbf{m L} \text { (Cryptococcus neoformans } \\
\text { ATCC } 90112 \text { ) } \\
\text { MIC } 200 \mu \mathrm{g} / \mathbf{m L} \text { (Cryptococcus neoformans } \\
\text { ATCC } 90112 \text { ) }\end{array}$ & {$[102]$} \\
\hline 57 & $\begin{array}{l}\text { Aspergillus sp. } \\
\text { ZA-01 }\end{array}$ & Marine & $\begin{array}{l}\mathrm{AB} \\
\mathrm{CT}\end{array}$ & $\begin{array}{l}\text { MIC } 25 \mu \mathrm{M} \text { (V. parahemolyticus, } V . \text { anguillarum, } \\
\text { V. alginolyticus) } \\
\text { IC }_{\mathbf{5 0}} \mathbf{1 . 8} \boldsymbol{\mu M}(\mathrm{A}-549)\end{array}$ & \\
\hline 58 & & & & $\begin{array}{l}\text { MIC } 1.56,1.56,3.12 \mu \mathrm{M} \text { (V. parahemolyticus, } \\
\text { V. anguillarum, V. alginolyticus) }\end{array}$ & \\
\hline 59 & & & & $\begin{array}{l}\text { MIC 6.25, } 25.0,25.0 \mu \mathrm{M} \text { (V. parahemolyticus, } \\
\text { V. anguillarum, V. alginolyticus) }\end{array}$ & \\
\hline 60 & & & & $\begin{array}{l}\text { MIC } 3.12,25.0,12.5 \mu \mathrm{M} \text { (V. parahemolyticus, } \\
\text { V. anguillarum, V. alginolyticus) }\end{array}$ & [96] \\
\hline 61 & & & & $\begin{array}{l}\text { MIC } 12.5,25.0,12.5 \mu \mathrm{M} \text { (V. parahemolyticus, } \\
\text { V. anguillarum, V. alginolyticus) }\end{array}$ & \\
\hline 62 & & & & $\begin{array}{l}\text { MIC } 6.25,6.25,12.5 \mu \mathrm{M} \text { (V. parahemolyticus, } \\
\text { V. anguillarum, V. alginolyticus) }\end{array}$ & \\
\hline 63 & & & & $\begin{array}{l}\text { MIC } 25.0,6.25,25.0 \mu \mathrm{M} \text { (V. parahemolyticus, } \\
\text { V. anguillarum, V. alginolyticus) }\end{array}$ & \\
\hline 72 & $\begin{array}{l}\text { Penicillium } \mathrm{sp} . \\
\text { ZZ380 } \\
\text { Penicillium citrinum } \\
\text { SCSIO } 41017\end{array}$ & Marine & $\mathrm{AF}$ & $\begin{array}{l}\text { Qualitative AF activity (C. albicans, S. fimicola, } \\
\text { A. furfuraceus) [109] }\end{array}$ & $\begin{array}{r}{[78]} \\
{[108]}\end{array}$ \\
\hline 73 & $\begin{array}{l}\text { Penicillium citrinum } \\
\text { SCSIO } 41017\end{array}$ & Marine & CT & $\begin{array}{l}\text { IC }_{50} \mathbf{1 6 . 0}-\mathbf{4 6 . 4} \boldsymbol{\mu M}(\mathrm{SF}-268, \mathrm{MCF}-7, \mathrm{HepG}-2, \\
\mathrm{A} 549)\end{array}$ & [108] \\
\hline 75 & $\begin{array}{l}\text { Colletotrichum } \\
\text { gloeosporioides }\end{array}$ & Mangrove & $\mathrm{AB}$ & $\begin{array}{l}\text { MIC 12.5, 25.0, } 25.0 \mu \mathrm{g} / \mathrm{mL} \text { (B. cereus, B. subti- } \\
\text { lis, S. aureus) }\end{array}$ & [113] \\
\hline $\begin{array}{l}92 \\
93 \\
\end{array}$ & $\begin{array}{l}\text { Penicillium citrinum } \\
\text { HL-5126 }\end{array}$ & Mangrove & $\begin{array}{l}\mathrm{AB} \\
\mathrm{CT}\end{array}$ & $\begin{array}{l}\text { MIC } 20.0 \mu \mathrm{g} / \mathrm{mL} \text { (S. aureus) } \\
\text { IC }_{\mathbf{5 0}} 15.7 \mu \mathrm{g} / \mathrm{mL}(\mathrm{A} 549)\end{array}$ & {$[124]$} \\
\hline 97 & Nigrospora sphaerica & Mangrove & $\begin{array}{l}\mathrm{CT} \\
\mathrm{AB} \\
\mathrm{AF}\end{array}$ & 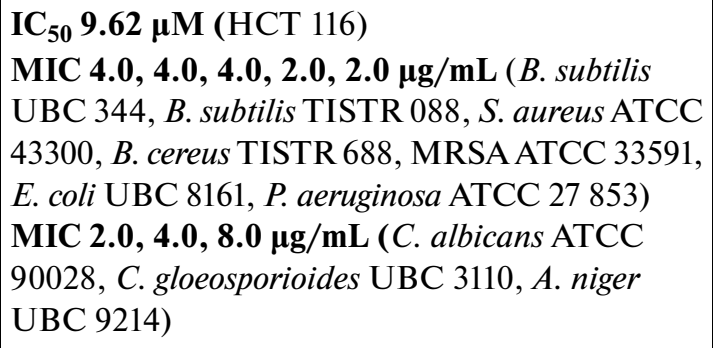 & [128] \\
\hline
\end{tabular}


Table 3. (Contd.)

\begin{tabular}{|c|c|c|c|c|c|}
\hline Cpd. & Producer/species & $\begin{array}{l}\text { Extremophile } \\
\text { type/origin }\end{array}$ & $\begin{array}{l}\text { Activity/ } \\
\text { toxicity }\end{array}$ & $\mathrm{EC}_{50}, \mathrm{IC}_{50}, \mathrm{MIC}$ & Ref. \\
\hline 95 & $\begin{array}{l}\text { Aspergillus sp. } \\
\text { YQ-13 }\end{array}$ & $\begin{array}{l}\text { Hydrothermal } \\
\text { vent sediments, } \\
\text { Kueishantao, } \\
\text { Taiwan/Thermo- } \\
\text { phile }\end{array}$ & $\mathrm{AB}$ & $\begin{array}{l}\text { MIC 860.8, 40.93 } \mu \mathrm{g} / \mathrm{mL} \text { ( } P \text {. aeruginosa, } \\
\text { B. subtilis) } \\
\text { MIC 394.1, 191.67, 58.21, 58.21 } \mu \mathrm{g} / \mathrm{mL} \\
\text { (P. aeruginosa, E. coli, B. subtilis, MRSA) }\end{array}$ & [126] \\
\hline $\begin{array}{l}68 \\
70 \\
56\end{array}$ & $\begin{array}{l}\text { Aspergillus sydowii } \\
\text { C1-S01-A7 }\end{array}$ & $\begin{array}{l}\text { Deep-sea } \\
4950 \mathrm{~m}\end{array}$ & $\begin{array}{l}\mathrm{AB} \\
\mathrm{CT}\end{array}$ & 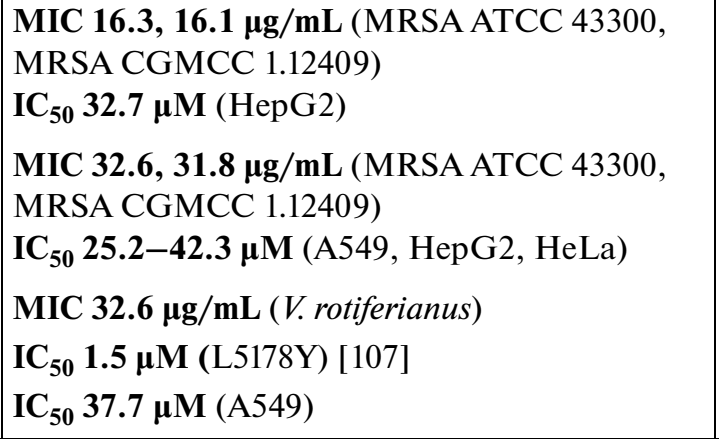 & [77] \\
\hline $\begin{array}{l}99 \\
55 \\
\end{array}$ & $\begin{array}{l}\text { Penicillium chrysoge- } \\
\text { num } \\
\text { MCCC 3A00292 }\end{array}$ & $\begin{array}{l}\text { Deep-sea } \\
2076 \mathrm{~m}\end{array}$ & CT & $\begin{array}{l}\mathrm{IC}_{50} \mathbf{1 0 . 2 1} \boldsymbol{\mu \mathrm { M }}(\mathrm{BIU}-87) \\
\mathrm{IC}_{\mathbf{5 0}} \mathbf{1 5 . 9 4} \boldsymbol{\mu \mathrm { M }}(\mathrm{BEL}-7402)\end{array}$ & [46] \\
\hline $\begin{array}{l}80 \\
81 \\
82\end{array}$ & $\begin{array}{l}\text { Penicillium sp. } \\
\text { SCSIO } 06720\end{array}$ & $\begin{array}{l}\text { Deep-sea } \\
4762 \mathrm{~m}\end{array}$ & $\begin{array}{l}\mathrm{CT} \\
\mathrm{AB} \\
\mathrm{AF}\end{array}$ & $\begin{array}{l}\text { IC }_{\mathbf{5 0}} \mathbf{1 1 . 7} \mathbf{9 9 9 . 0} \boldsymbol{\mu M}(\mathrm{A} 375, \mathrm{~A} 549, \mathrm{HeLa}, \\
\text { HepG2, SW-620, L-02) [111] } \\
\text { MIC 10.4, 46.9 } \boldsymbol{\mu g} / \mathbf{m L}(\text { S. aureus ATCC 29213, } \\
\text { MRSA-shh-1) } \\
\text { IC }_{\mathbf{5 0}} \mathbf{4 0 . 0}, \mathbf{5 0 . 0} \boldsymbol{\mu g} / \mathbf{m L}(\mathrm{A} 549, \text { HeLa) [112] }\end{array}$ & [59] \\
\hline 89 & $\begin{array}{l}\text { Cochliobolus lunatus } \\
\text { SCSIO41401 }\end{array}$ & Marine & $\begin{array}{l}\mathrm{CT} \\
\mathrm{AB}\end{array}$ & 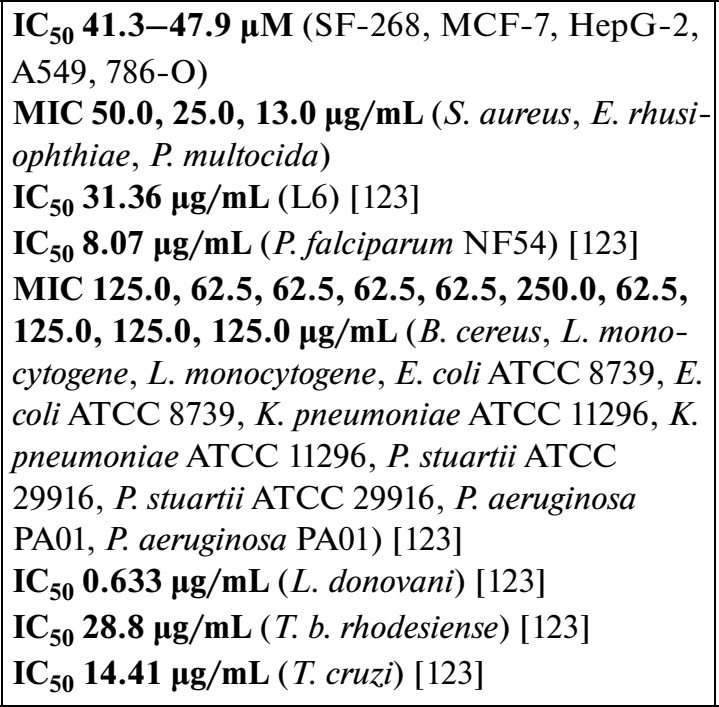 & [121] \\
\hline $\begin{array}{r}109 \\
110 \\
96\end{array}$ & $\begin{array}{l}\text { Penicillium pinophi- } \\
\text { lum } \\
\text { SCAU037 }\end{array}$ & Mangrove & CT & 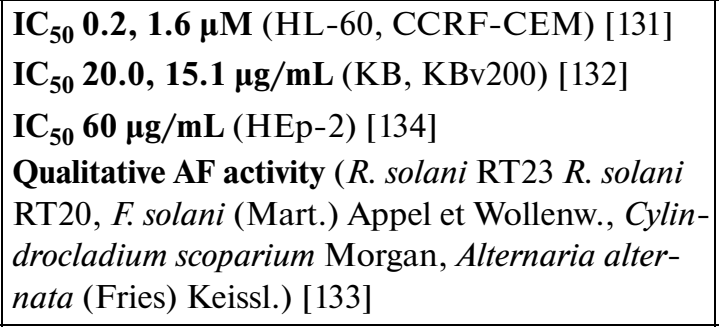 & {$[130]$} \\
\hline 79 & $\begin{array}{l}\text { Cladosporium sp. } \\
\text { OUCMDZ-302 }\end{array}$ & Mangrove & CT & $\begin{array}{l}\text { IC }_{50} 40 \mu \mathrm{M}(\mathrm{HL}-60, \mathrm{SMMC}-7721, \mathrm{~A}-549, \\
\mathrm{MCF}-7, \mathrm{SW} 480)[117]\end{array}$ & {$[116]$} \\
\hline
\end{tabular}




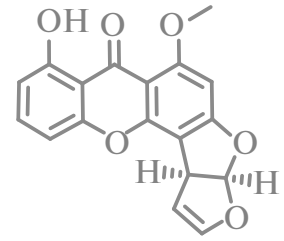

Stergmatocystin

(54)

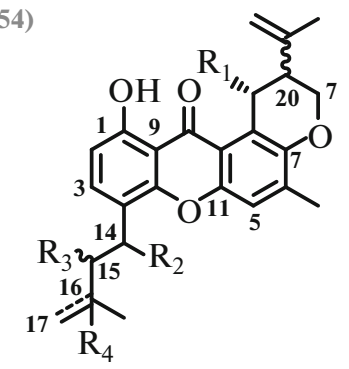

Aspergixanthone A

(57) $20(S), 15(R) \mathrm{R}_{1}=\mathrm{OAc}, \mathrm{R}_{2}=\mathrm{OCH}_{3}, \mathrm{R}_{3}=\mathrm{OH}, \mathrm{R}_{4}=\mathrm{OH}$

Aspergixanthones $\mathrm{I}-\mathrm{K}$

(58) $20(R), 15(S) \mathrm{R}_{1}=\mathrm{OH}, \mathrm{R}_{2}=\mathrm{H}, \mathrm{R}_{3}=\mathrm{OAc}, \mathrm{R}_{4}=\mathrm{OH}$

(59) $20(S), 15(R) \mathrm{R}_{1}=\mathrm{OAc}, \mathrm{R}_{2}=\mathrm{OCH}_{3}, \mathrm{R}_{3}=\mathrm{OH}, \mathrm{R}_{4}=\mathrm{H}, \Delta^{16-17}$

(60) $20(S), 15(R) \mathrm{R}_{1}=\mathrm{OH}, \mathrm{R}_{2}=\mathrm{OCH}_{3}, \mathrm{R}_{3}=\mathrm{OH}, \mathrm{R}_{4}=\mathrm{H}, \Delta^{16-17}$

15-Acetyl tajixanthone hydrate

(61) $20(S), 15(S) \mathrm{R}_{1}=\mathrm{OH}, \mathrm{R}_{2}=\mathrm{H}, \mathrm{R}_{3}=\mathrm{OAc}, \mathrm{R}_{4}=\mathrm{OH}$

Tajixanthone hydrate

(62) $20(S), 15(S) \mathrm{R}_{1}=\mathrm{OH}, \mathrm{R}_{2}=\mathrm{H}, \mathrm{R}_{3}=\mathrm{OH}, \mathrm{R}_{4}=\mathrm{OH}$

16-Chlorotajixanthone

(63) $20(S), 15(S) \mathrm{R}_{1}=\mathrm{OH}, \mathrm{R}_{2}=\mathrm{H}, \mathrm{R}_{3}=\mathrm{OH}, \mathrm{R}_{4}=\mathrm{CI}$<smiles></smiles>

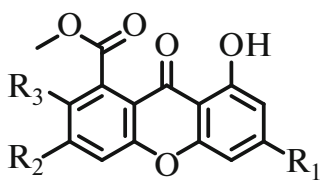

3-Hydroxy pinselin

(64) $\mathrm{R}_{1}=\mathrm{CH}_{3}, \mathrm{R}_{2}=\mathrm{OH}, \mathrm{R}_{3}=\mathrm{OH}$

Chloroisosuloerin dehydrate

(65) $\mathrm{R}_{1}=\mathrm{CH}_{3}, \mathrm{R}_{2}=\mathrm{OCH}_{3}, \mathrm{R}_{3}=\mathrm{H}$

(66) $\mathrm{R}_{1}=\mathrm{CH}_{3}, \mathrm{R}_{2}=\mathrm{H}, \mathrm{R}_{3}=\mathrm{OH}$

(67) $\mathrm{R}_{1}=\mathrm{CH}_{2} \mathrm{OAc}, \mathrm{R}_{2}=\mathrm{H}, \mathrm{R}_{3}=\mathrm{H}$

Sydowinin B

(68) $\mathrm{R}_{1}=\mathrm{CH}_{2} \mathrm{OH}, \mathrm{R}_{2}=\mathrm{H}, \mathrm{R}_{3}=\mathrm{OH}$

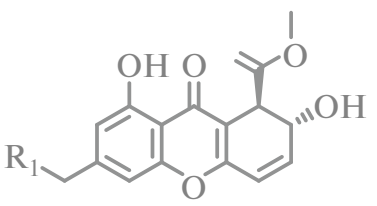

Aspergillusone A
(69) $\mathrm{R}_{1}=\mathrm{H}$
AGI-B4
(70) $\mathrm{R}_{1}=\mathrm{OH}$
2 -O-acetyl-AGI-B4
(71) $\mathrm{R}_{1}=$ OAc

Fig. 6. Xanthones and dihydroxanthones.

mones. Among the new compounds of this class, we can note the previously undescribed pyranochromone dimer (86) isolated from a marine fungus of the Penicillium genus [114], which did not show antibiotic properties.

As for the previously described dimeric chromones, the data on biological activity has been expanded for some of them. For example, significant cytotoxic activity was detected for the 4,4'-bound secalonic acid D isomer (85) isolated from marine micromycetes of the Penicillium genus [118], which was known primarily for its bactericidal properties [119]. The stereoisomer of secalonic acid D (88) [120], which also showed the cytotoxic activity, was found in a mangrove fungus of the Cladosporium genus [121]. Another related compound, secalonic acid A (87) [122], was isolated from a marine fungus of the Penicillium genus [114]. The previously described xan- thone purpureone (89) [76, 123] showed cytotoxic activity [76].

Along with the xanthones and quinones described above, the study of secondary metabolites of micromycetes revealed structurally similar compounds, primarily benzophenones. For example, a micromycete of mangrove origin belonging to the Penicillium genus [124] became the source of two new benzophenones, i.e., the antibacterial penibenzophenone A (92) and the cytotoxic penibenzophenone B (93), along with the wellknown benzophenone sulochrine (90) [70, 125].

Another example is the thermophilic micromycete Aspergillus sp. YQ-13 [126] isolated from hydrothermal vents. It has been studied in terms of the spectrum of secondary metabolites. A new natural compound, methyl ester of 3-hydroxy-2-(2-hydroxy-6-methoxy4-methylbenzoyl)-5-methoxy benzoic acid (94), showed low antibacterial activity. At the same time, a 
<smiles>[R3]c1cc([R])c2c(c1)O[C@H]1CCC([R])C1=C2C</smiles>

Coniochaetones B, M, L

(72) $1(S) \mathrm{R}_{1}=\mathrm{CH}_{3}, \mathrm{R}_{2}=\mathrm{OH}, \mathrm{R}_{3}=\mathrm{OH}$

(73) $1(S) \mathrm{R}_{1}=\mathrm{OH}, \mathrm{R}_{2}=\mathrm{OCH}_{3}, \mathrm{R}_{3}=\mathrm{COOH}$

(74) $1(R) \mathrm{R}_{1}=\mathrm{OH}, \mathrm{R}_{2}=\mathrm{O} \mathrm{CH}_{3}, \mathrm{R}_{3}=\mathrm{COOH}$

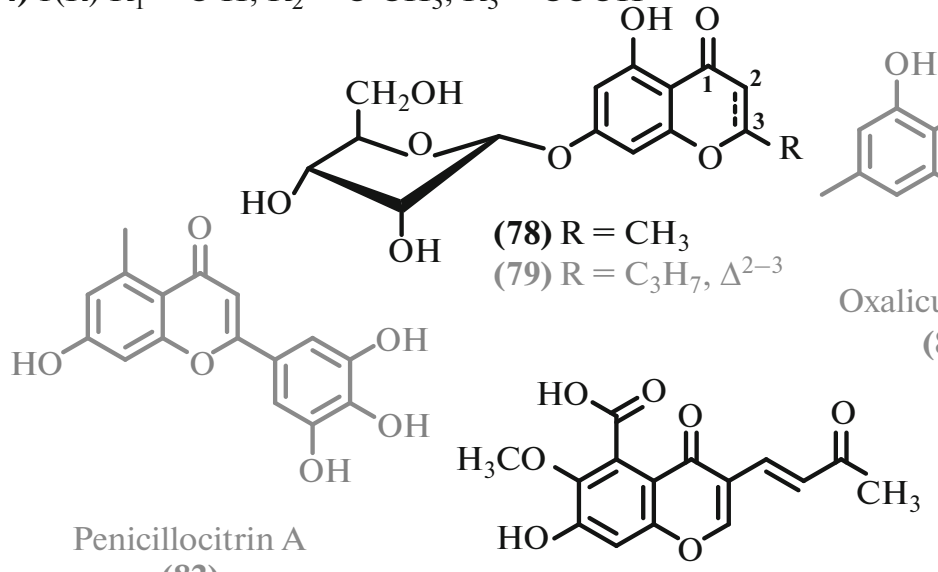<smiles>COc1ccc(O)c2c1O[C@]1(C(=O)O)C(=C(O)C[C@H](C)[C@H]1O)C(=O)c1c(-c3ccc(O)c4c3O[C@]3(C(=O)O)C(=C(O)C[C@H](C)[C@H]3O)C4=O)ccc(O)c1-2</smiles><smiles>COc1cc(OC)c2c(c1O)C(=O)C1=C(O)CC[C@H](O)[C@]1(OC(C)=O)O2</smiles>

Purpureone

(89)<smiles>CC(=O)[C@H]1C(=O)C[C@]23COc4cc(O)c(O)cc4C(=O)[C@@H]2[C@H](O3)c2c(oc3cc(O)c(O)cc3c2=O)CC1(C)C</smiles>

(83)<smiles>C[C@H]1CC(=O)c2c(ccc(O)c2O)O[C@@H]1C</smiles>

(75)<smiles>CC1=CC(=O)[C@]2(COc3cc(O)c(O)cc3C2=O)C1</smiles>

Spirofuranochromone

(77)

(80)<smiles>COC(=O)[C@]12Oc3ccc(-c4ccc5c(c4O)C(=O)C4=C(O)C[C@@H](C)[C@H](O)[C@]4(C(C)=O)O5)c(O)c3C(=O)C1=C(O)C[C@@H](C)[C@H]2O</smiles>

Secalonic acid A

\section{Secalonic acid D}

(88)

Fig. 7. Chromones and dimeric compounds.

well-known metabolite, 1,2-SECO-trypacidin (95), showed a noticeable activity [127].

A new antibiotic, nigronaphthaphenyl (97), isolated from a mangrove endophyte [128], showed a wide range of biological activity including anti-inflammatory properties and the ability to inhibit alpha-glucosidase, which made it promising for further study.
The study of some extremophilic micromycetes led to the isolation of known benzophenones. Thus, the screening of marine micromycetes for antibacterial activity revealed the producer strain of six known metabolites [74] including benzophenone sulochrine (90) $[70,125]$. Another described benzophenone, chloroisosulochrin (91) [103], was isolated in the 
study of secondary metabolites of a fungus of the Pseudopestalotiopsis genus [102].

In addition to discovery new benzophenones, the study of secondary metabolites of extremophilic micromycetes has led to the isolation of several structurally similar compounds. A new chromene, 1-hydroxy-12-methoxycitromycin (103), was isolated from a marine fungus of the Penicillium genus [114], along with a known analog, citromycin (102) [129].

Seafloor fungus Penicillium chrysogenum MCCC 3A00292 [46] produces a series of related compounds, i.e., previously undescribed peniciversioles A-C (99101), which were studied as cytotoxic agents. Interestingly, peniciversiole A (99) showed cytotoxic properties in contrast to peniciversioles B and C $(\mathbf{1 0 0}, \mathbf{1 0 1})$.

Five new funicon derivatives named pinophilones A-E (104-108) were isolated from soil mangrove producer Penicillium pinophilum SCAU037 [130] along with eighteen biosynthetically related known compounds. Some of these compounds, i.e., penisimplicissin (109) [131], methoxyvermistatin (110) [132], and 3-O-methylfunicon (96) [133, 134] showed high antibiotic activity.

\subsection{Other Polyketides}

In addition to the compounds described above, extremophilic micromycetes became the source of some other polyketides during the studied period. Several structural families can be distinguished among isolated compounds. Isocoumarins (111-120), dihydroisocoumarins (121-127), and other lactones (128-148) can be distinguished among cyclic esters. Most of the new and known metabolites can be assigned to phenols and their derivatives (149-176) and diphenyl esters (177-189). Many isolated metabolites were aromatic compounds, i.e., phenalenones (190-198), grisane metabolites (199-201), and azaphilones (202-222). Linear polyketides with various side chains (223-245) and other polyketides (246-257) were also obtained.

Isocumarins and their derivatives are common bioactive metabolites of micromycetes [135]. New isocoumarins were also isolated from mangrove micromycete Ascomycota sp. [136], endophyte Pluchea indica. This strain was the source of three new isocoumarins, i.e., dichlorodiaportin (116), desmethyldichlorodiaportin (117), and desmethyldichlorodiaportinol (114), along with several other well-known analogs, i.e., dichlorodiaportinol (115) [137], desmethyldichlorodiaportin (119) [138], and dichlorodiaportin (120) [139, 140]. Other isocoumarins with antibiotic activity turned out to have been be previously described. For example, the chemical study of mangrove endophyte Phyllosticta capitalensis [141] afforded the known isocoumarin derivative with antibiotic activity, 6,8-dihydroxy-5-methoxy-3-methyl$1 H$-isochromen-1-one (118) [142]. When studying the Alternaria sp. SCSIO41014 strain [87], researchers obtained the isocoumarin derivatives, i.e., alternariol (111) [143], 5- $O$-methyl ester of alternariol (112) [143], and altenuisol (113) [144].

Several new compounds isolated from extremophilic micromycetes belong to dihydroisocoumarins. A new isocumarin derivative, pestalotiopisorin B (121) with moderate antibacterial activity, was isolated from the Pestalotiopsis strain of mangrove origin [145]. Another new dihydroisocoumarin, nordihydroaltenuene A (123), and known bioactive analogs, i.e., (-)-3$O$-acetylaltenuene (126) [143, 146], altenusin (155) [147], and dihydroaltenuene A (127) [148], were isolated in the study of the Alternaria sp. SCSIO41014 strain [87]. Several known dihydrocoumarins were obtained from other sources. $(3 R, 4 S)$-6,8-Dihydroxy3,4,7-trimethylisocoumarin (122) [149] was isolated from a deep-sea micromycete [59]; cladosporin (124) and 5'-hydroxyasperentin (125) [150] were isolated from a marine fungus [151].

In addition to isocumarin derivatives, a series of lactones of various natures were isolated when studying extremophilic micromycetes (Fig. 10). Of the new lactones, four new unusual sulfur-containing compounds should be noted, which were isolated from the mangrove Cladosporium micromycete [152]. The lactones were called thiocladospolids A-D (128, 130132). Similar known antibiotic pandangolide 3 was also isolated (129) [152, 153]. Comparison of the NMR data of new macrolides and known pandangolide 3 (129) allowed to revise its structure and clarify the attachment site of the side sulfide chain. Another new 10-membered lactone decarestrictine Q (135), an analog of decarestrictin B (136) [154], was isolated from a marine fungus of the Pseudopestalotiopsis genus [102]. Significant cytotoxicity was shown for another structurally original depsidone, curdepsidone A (139), isolated from the strain of the Curvularia genus of the marine origin [155]. A new depsidone derivative, botryorhodine I (141), and eight known compounds were isolated from the soil mangrove micromycete Lasiodiplodia theobromae M4.2-2 [156]. Several other antibiotics were also found, i.e., antifungal and cytotoxic botryorhodines A, B, and D (142-144) [157] and compounds (140) and (134) [156, 158]. Mangrove endophyte Colletotrichum gloeosporioides [113] became the source of new lactone (148), which showed antimicrobial activity.

The approach to activation of silent biosynthetic clusters of micromycetes deserves a special mention: biosynthesis of a new cytotoxic polyketide, purpurogenic acid (145), was observed in a mutant strain of Penicillium purpurogenum G59 obtained by exposure of diethyl sulfate [159].

In addition to the new compounds, some previously described biologically active lactones were obtained. A well-known aromatic lactone, 8-dimethoxy-10-methoxy-ventiquinone C (137), was iso- 


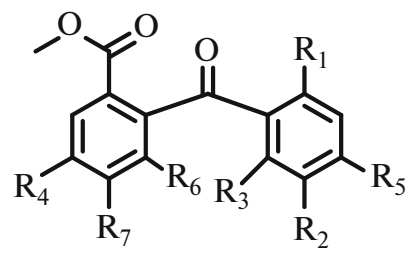

Sulochrin

(90) $\mathrm{R}_{1}=\mathrm{OH}, \mathrm{R}_{2}=\mathrm{H}, \mathrm{R}_{3}=\mathrm{OH}, \mathrm{R}_{4}=\mathrm{OH}, \mathrm{R}_{5}=\mathrm{CH}_{3}, \mathrm{R}_{6}=\mathrm{OCH}_{3}, \mathrm{R}_{7}=\mathrm{H}$

3-O-Methylfunicon

Chloroisosulochrin

(91) $\mathrm{R}_{1}=\mathrm{OH}, \mathrm{R} 2=\mathrm{H}, \mathrm{R}_{3}=\mathrm{OH}, \mathrm{R}_{4}=\mathrm{OCH}_{3}, \mathrm{R}_{5}=\mathrm{CH}_{3}, \mathrm{R}_{6}=\mathrm{OH}, \mathrm{R}_{7}=\mathrm{Cl}$

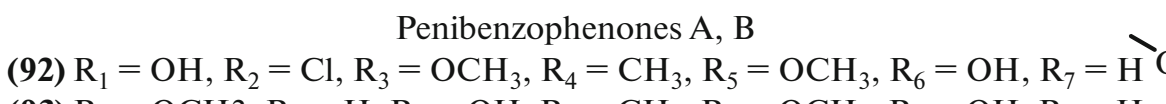

(93) $\mathrm{R}_{1}=\mathrm{OCH} 3, \mathrm{R}_{2}=\mathrm{H}, \mathrm{R}_{3}=\mathrm{OH}, \mathrm{R}_{4}=\mathrm{CH}_{3}, \mathrm{R}_{5}=\mathrm{OCH}_{3}, \mathrm{R}_{6}=\mathrm{OH}, \mathrm{R}_{7}=\mathrm{H}$

(94) $\mathrm{R}_{1}=\mathrm{OCH}_{3}, \mathrm{R}_{2}=\mathrm{H}, \mathrm{R}_{3}=\mathrm{OH}, \mathrm{R}_{4}=\mathrm{OCH}_{3}, \mathrm{R}_{5}=\mathrm{CH}_{3}, \mathrm{R}_{6}=\mathrm{OH}, \mathrm{R}_{7}=\mathrm{H}$

(95) $\mathrm{R}_{1}=\mathrm{OCH}_{3}, \mathrm{R}_{2}=\mathrm{H}, \mathrm{R}_{3}=\mathrm{OH}, \mathrm{R}_{4}=\mathrm{OH}, \mathrm{R}_{5}=\mathrm{CH}_{3}, \mathrm{R}_{6}=\mathrm{OCH} 3, \mathrm{R}_{7}=\mathrm{Cl}$<smiles>COc1cc(OC)c2cc(C(=O)c3ccccc3)ccc2c1O[18O]</smiles><smiles>CC(C)C1CCCC1</smiles>

(97)<smiles>CC(C)=CC[C@]1(O)C2=C(C[C@H](O)[C@@H]1O)C(=O)CC(C)(C)O2</smiles>

Pseudopestalone

(98)<smiles>[R]c1cc(=O)c2c(o1)-c1c(cc(O)c([R])c1[R])OC2</smiles>

Citromycin

(102) $\mathrm{R}_{1}=\mathrm{OH}, \mathrm{R}_{2}=\mathrm{H}, \mathrm{R}_{3}=\mathrm{Me}$

1-Hydroxy-12-methoxycitromycin (103) $\mathrm{R}_{2}=\mathrm{OMe}, \mathrm{R}_{2}=\mathrm{H}, \mathrm{R}_{3}=\mathrm{Me}$<smiles>[R]C1([R2])Cc2occ(C(=O)c3c(OC)cc(OC)cc3C(=O)OC)c(=O)c2O1</smiles>

Pinophilones A, B (104) $\mathrm{R}_{1}=\mathrm{H}, \mathrm{R}_{2}=\mathrm{Me}$ (105) $R_{1}=M e, R_{2}=H$

Peniciversioles A-C (99) $\mathrm{R}_{1}=\mathrm{OH}, \mathrm{R}_{2}=\mathrm{H}, \Delta^{12-13}$

(100) $\mathrm{R}_{1}=\mathrm{OH}, \mathrm{R}_{2}=\mathrm{H}$

(101) $\mathrm{R}_{1}=\mathrm{H}, \mathrm{R}_{2}=\mathrm{OH}$<smiles>[R]c1cc(OC)c2c(c1[R])C(=O)O[C@H]2c1coc([R4])c([R])c1=O</smiles>

Pinophilones C-E

(106) $\mathrm{R}_{1}=\mathrm{OH}, \mathrm{R}_{2}=\mathrm{R}_{3}=\mathrm{H}, \mathrm{R}_{4}=n \mathrm{Pr}$

(107) $\mathrm{R}_{1}=\mathrm{OH}, \mathrm{R}_{2}=\mathrm{H}, \mathrm{R}_{3}=\mathrm{OMe}, \mathrm{R}_{4}=n \operatorname{Pr}$

(108) $\mathrm{R}_{1}=\mathrm{OH}, \mathrm{R}_{2}=\mathrm{OMe}, \mathrm{R}_{3}=\mathrm{H}, \mathrm{R}_{4}=$ propenyl

Penisimplicissin

(109) $R_{1}=$ OMe, $R_{2}=R_{3}=H, R_{4}=M e$

Methoxyvermistatin

(110) $\mathrm{R}_{1}=\mathrm{OMe}, \mathrm{R}_{2}=\mathrm{H}, \mathrm{R}_{3}=\mathrm{OMe}, \mathrm{R}_{4}=$ propenyl

Fig. 8. Benzophenones and other related compounds.

lated from a fungus of marine origin [108] [77, 160]. 8Dimethoxy-10-methoxyventriquinone C (137) was also isolated from seafloor micromycete Aspergillus sydowii C1-S01-A7 [77]. Several discovered lactones have been previously described, including alterlactone (147) [143] isolated in the study of the Alternaria sp. SCSIO41014 strain [87], the antibiotic lactone penicillide (138) [161] isolated from mangrove soil producer Penicillium pinophilum SCAU037 [130], nod- ulisporone A (146) [162] found in the study of deep micromycete [59], and asperdemin (133) [163] isolated from deep-sea fungus Penicillium chrysogenum MCCC 3A00292 [46].

A significant proportion of antibiotic compounds isolated from extremophilic micromycetes are phenols and their derivatives, many of which have been described for the first time (Fig. 11). The study of secondary metabolites of the Zopfiella marina strain [164] 
<smiles>[R]c1cc(O)c2c(=O)oc3c([R])c(O)cc(C)c3c2c1</smiles>

Alternariol

(111) $\mathrm{R}_{1}=\mathrm{OH}, \mathrm{R}_{2}=\mathrm{H}$

(112) $\mathrm{R}_{1}=\mathrm{OMe}, \mathrm{R}_{2}=\mathrm{OH}$<smiles>COc1cc(O)c2c(c1)[C@H]1[C@H](O)[C@@H](O)C[C@]1(C)OC2=O</smiles>

Nordihydroaltenuene A

(123)

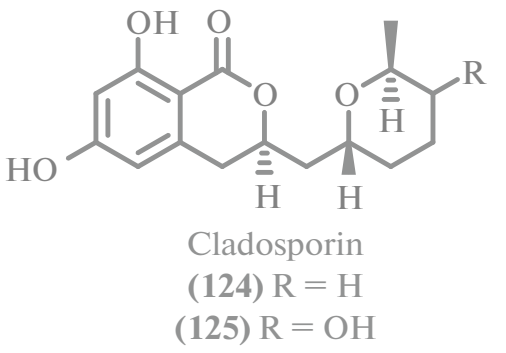<smiles>COc1cc(O)c2c(=O)oc3cc(O)c(O)cc3c2c1</smiles><smiles>[R4]c1cc2c(c(O)c1C)C(=O)OC([R])([R])[C@@]2([R])C</smiles>

Pestalotiopisorin B<smiles>[R]Oc1cc(O)c2c(=O)oc([R3])cc2c1[R2]</smiles><smiles>C#C[C@@H](O)[C@@H](O)C(Cl)Cl</smiles>

(114) $R_{1}=H, R_{2}=H, R_{3}=A$ (115) $\mathrm{R}_{1}=\mathrm{CH}_{3}, \mathrm{R}_{2}=\mathrm{H}, \mathrm{R}_{3}=\mathrm{A}$ (116) $\mathrm{R}_{1}=\mathrm{CH}_{3}, \mathrm{R}_{2}=\mathrm{H}, \mathrm{R}_{3}=\mathrm{B}$ (117) $R_{1}=H, R_{2}=H, R_{3}=B$ (118) $\mathrm{R}_{1}=\mathrm{H}, \mathrm{R}_{2}=\mathrm{OCH}_{3}, \mathrm{R}_{3}=\mathrm{CH}_{3}$

Desmethyldichlorodiaportin

(119) $\mathrm{R}_{1}=\mathrm{H}, \mathrm{R}_{2}=\mathrm{H}, \mathrm{R}_{3}=\mathrm{C}$

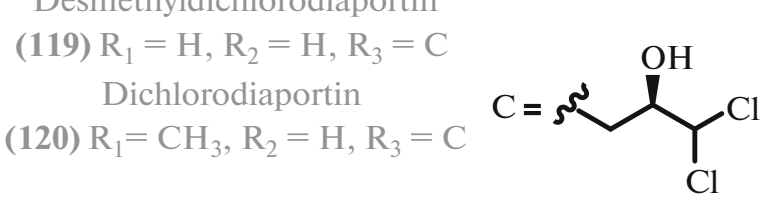<smiles>C=CC[C@@]1(C(Cl)Cl)C[C@@H](O)C(=O)O1</smiles>
(121) $\mathrm{R}_{1}=\mathrm{OH}, \mathrm{R}_{2}=\mathrm{H}, \mathrm{R}_{3}=\mathrm{CH}_{3}, \mathrm{R}_{4}=\mathrm{H}$ (122) $\mathrm{R}_{1}=\mathrm{H}, \mathrm{R}_{2}=\mathrm{CH}_{3}, \mathrm{R}_{3}=\mathrm{H}, \mathrm{R}_{4}=\mathrm{OH}$

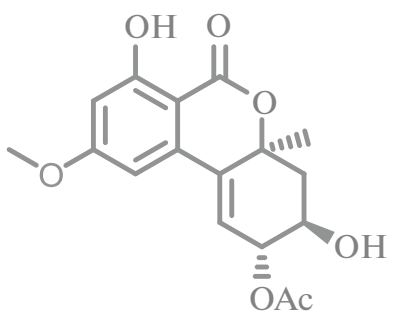

(-)-Altenuene-3-acetoxy ester (126)

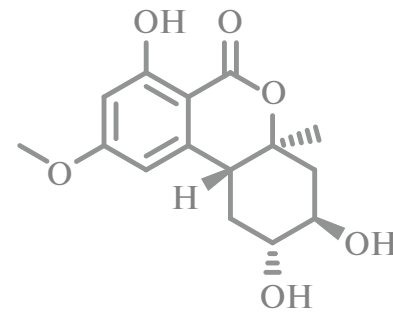

Dihydroaltenuene A (127)

Fig. 9. Isocoumarins and dihydroisocoumarins.

of marine origin allowed to identify several previously undescribed compounds, i.e., two derivatives of salicylic aldehyde $(\mathbf{1 6 1}, \mathbf{1 6 5})$, five dihydroisobenzofurans (171-175), and 5-chloro-3-deoxyizoochracinic acid (176). Of these, only the derivative (161) showed the antibacterial, including antituberculosis, activity.

The spectrum of secondary metabolites of the mangrove fungus of the Dothiorella genus [165] includes some phenols with an antibiotic activity, which primarily belong to the structural family of dothiorelones. New dothiorelones K-M (152-154) and known cytosporon derivatives, i.e., dothiorelones A (149) [166], B (150), and I (151) [167] and cytosporon A (160) were isolated [168].

New polyketides, sarcopodinols A and B (167, 168) were isolated from the Sarcopodium fungus [169], which was found as a result of physical and chemical screening of 60 strains of deep-sea micromycetes. Interestingly, the compound (168) showed a broader spectrum of cytotoxic action as compared to com- pound (167), which indicates the importance of the 5 '-OH group for the activity in some cell lines.

Bioactivity-based separation of a culture liquid extract of a thermophilic micromycete of the Penicillium genus [73], isolated from a hot spring in Saudi Arabia, resulted in a new phenol (162). 5-(2-Hydroxypropyl)-2,6-dimethylresorcinol (159), which did not exhibit biological activity, was isolated from the seafloor micromycete [59].

Mangrove endophytes have also proved to be a valuable source of biologically active compounds of this type. A new polyketide (166) was isolated from the Colletotrichum gloeosporioides strain [113], in addition to the abovementioned benzophenone (75) and lactone (148). The mangrove endophyte Cladosporium sp. [116] became the source of a series of new phenols $(163,164)$. A new prenylated benzaldehyde dioxoauroglaucin (156) was isolated from mangrove fungus Aspergillus sp. AV-2 [170], along with its several known analogs, of which flavoglaucin (157) [171], previously 


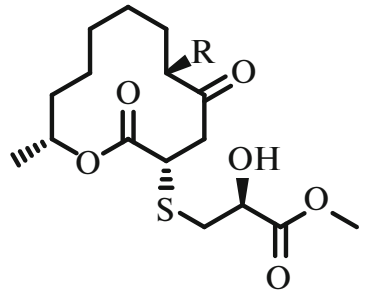

Thiocladospolid A (128) $\mathrm{R}=\mathrm{H}$ (129) $\mathrm{R}=\mathrm{OH}$<smiles>Cc1cc2c(c(=O)o1)C[C@H]1[C@H](O)CC(=O)O[C@](C)(C[C@H]1O)[C@H]2O</smiles>

Asperdemin

(133)<smiles>COC(=O)[C@H](O)CS[C@H](CC(=O)CCCCC[C@@H](C)O)C(=O)O</smiles>

Thiocladospolid B

(130)<smiles>C[C@H]1/C=C\CCCCCc2cc(O)cc(O)c2C(=O)O1</smiles>

(134)<smiles>COC(=O)CS[C@H](CC(=O)O)C(=O)O[C@@H](C)CCCCCCC(=O)O</smiles>

Thiocladospolid C

(131)<smiles>COC(=O)[C@H](O)CS[C@H](C[C@@H](O)[C@H](O)CCCCC[C@H](C)O)C(=O)O</smiles>

Thiocladospolid D

(132)<smiles>C[C@H]1C[C@H]2O[C@H]2C(=O)C[C@H](O)CC(=O)O1</smiles>

Decarestrictine Q

(135)<smiles></smiles>

Decarestrictine B (136)<smiles>[R]c1ccc2c(=O)oc3cc(CO)cc(O)c3c(=O)c2c1OC</smiles>

(137) $\mathrm{R}=\mathrm{H}$<smiles>[R]c1c([R])c([R])c2c(c1[R])OC(=O)c1c([R])c([R])c([R])c([R])c1O2</smiles>

Curdepsidone A

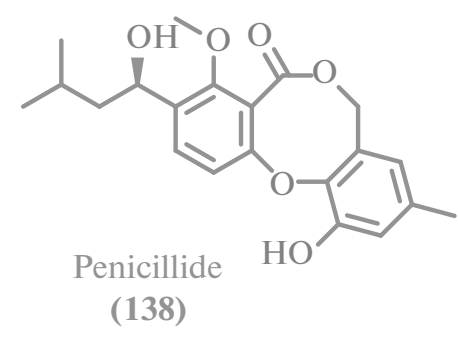

(138)

(139) $\mathrm{R}_{1}=\mathrm{OH}, \mathrm{R}_{2}=\mathrm{CHO}, \mathrm{R}_{3}=\mathrm{OCH}_{3}, \mathrm{R}_{4}=\mathrm{H}, \mathrm{R}_{5}=\mathrm{CH}_{3}, \mathrm{R}_{6}=\mathrm{H}, \mathrm{R}_{7}=\mathrm{OH}, \mathrm{R}_{8}=\mathrm{H}$

(140) $\mathrm{R}_{1}=\mathrm{CH}_{3}, \mathrm{R}_{2}=\mathrm{H}, \mathrm{R}_{3}=\mathrm{OH}, \mathrm{R}_{4}=\mathrm{CH}_{2} \mathrm{OCH}_{3}, \mathrm{R}_{5}=\mathrm{H}, \mathrm{R}_{6}=\mathrm{OH}, \mathrm{R}_{7}=\mathrm{H}, \mathrm{R}_{8}=\mathrm{CH}_{3}$

(141) $\mathrm{R}_{1}=\mathrm{CH}_{3}, \mathrm{R}_{2}=\mathrm{H}, \mathrm{R}_{3}=\mathrm{OH}, \mathrm{R}_{4}=\mathrm{CH}_{2} \mathrm{OH}, \mathrm{R}_{5}=\mathrm{OH}, \mathrm{R}_{6}=\mathrm{OH}, \mathrm{R}_{7}=\mathrm{H}, \mathrm{R}_{8}=\mathrm{CH}_{3}$

Botryorhodines A, B, D

(142) $\mathrm{R}_{1}=\mathrm{CH}_{3}, \mathrm{R}_{2}=\mathrm{H}, \mathrm{R}_{3}=\mathrm{OH}, \mathrm{R}_{4}=\mathrm{CHO}, \mathrm{R}_{5}=\mathrm{H}, \mathrm{R}_{6}=\mathrm{OH}, \mathrm{R}_{7}=\mathrm{H}, \mathrm{R}_{8}=\mathrm{CH}_{3}$

(143) $\mathrm{R}_{1}=\mathrm{CH}_{3}, \mathrm{R}_{2}=\mathrm{H}, \mathrm{R}_{3}=\mathrm{OH}, \mathrm{R}_{4}=\mathrm{CHO}, \mathrm{R}_{5}=\mathrm{CH}, \mathrm{R}_{6}=\mathrm{OH}, \mathrm{R}_{7}=\mathrm{H}, \mathrm{R}_{8}=\mathrm{CH}_{3}$

(144) $\mathrm{R}_{1}=\mathrm{CH}_{3}, \mathrm{R}_{2}=\mathrm{H}, \mathrm{R}_{3}=\mathrm{OH}, \mathrm{R}_{4}=\mathrm{CH}_{2} \mathrm{OH}, \mathrm{R}_{5}=\mathrm{H}, \mathrm{R}_{6}=\mathrm{OH}, \mathrm{R}_{7}=\mathrm{H}, \mathrm{R}_{8}=\mathrm{CH}_{3}$<smiles>O=C(O)/C=C/CCCC[C@H]1OC(=O)C(CO)=C1C(=O)O</smiles>

Purpurogenic acid

(145)

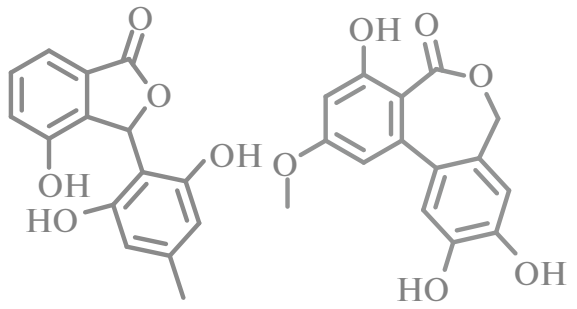

Nodulisporone A

(146)<smiles>C/C=C/c1cc(CC)c(O)c(=O)o1</smiles>

(148)

Fig. 10. Lactones.

known primarily as an antioxidant, showed a pronounced cytotoxic activity.

Previously described phenols were also revealed. When studying mangrove fungi of the Cladosporium genus [121], known polyketides were obtained, i.e., cladosporol E (170) [172], which previously did not show noticeable activity, and its more active analog cladosporol C (169) [173]. Chemical study of the 
<smiles>[R]CCCC(=O)c1c(O)cc(O)cc1CC(=O)OCC</smiles><smiles>CCCCC(O)CCCC</smiles>

Dothiorelone

(149)<smiles>[Z6]C(=O)OC</smiles>

Dothiorelone K (152)

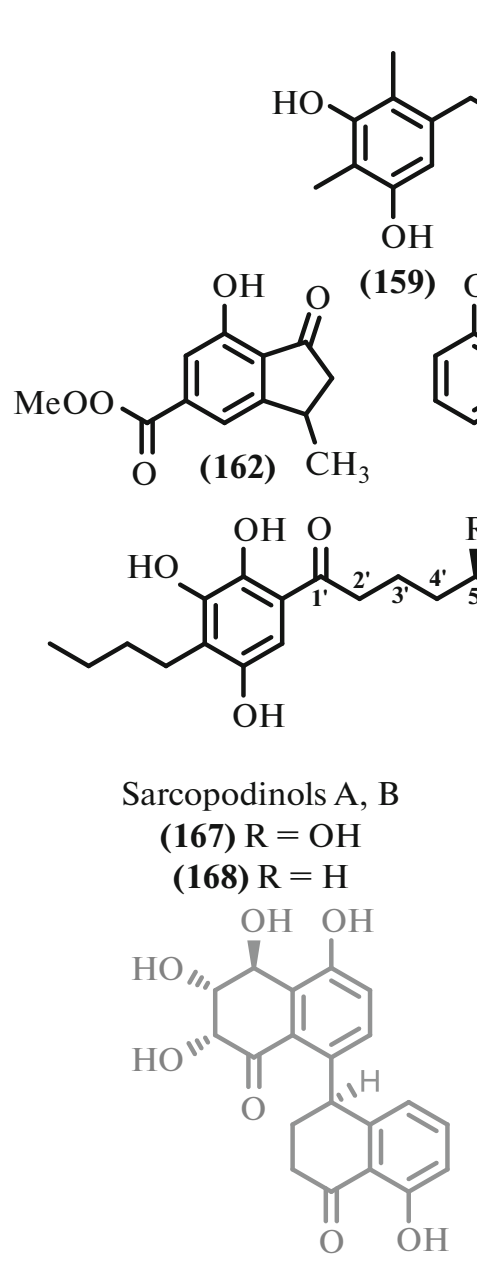

Ladosporol E

(170)

(150)

(163)<smiles>COc1cc(O)c(C(=O)O)c(-c2cc(O)c(O)cc2C)c1</smiles>

Altenusin

(155)<smiles>CC(C)=CCc1cc(O)c(/C=C\C2C=CC(C)OO2)c(C=O)c1O</smiles>

Dioxoauroglaucin

(156)

(151)

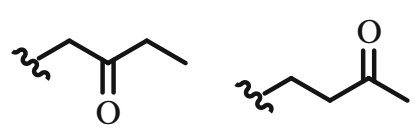

Dothiorelone L Dothiorelone M

(153)

(154)<smiles>CCc1c(O)cc(CC=C(C)C)c(C)c1O</smiles>

Flavoglaucin

(157)

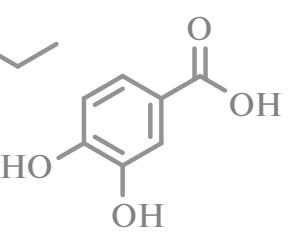

3,4-Dihydroxybenzoic acid

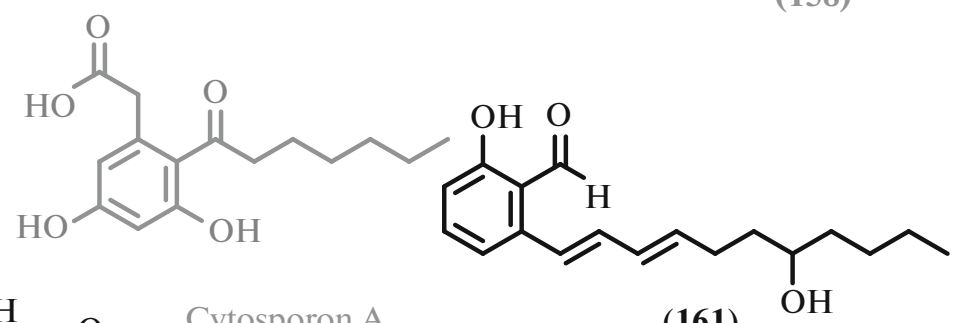

(161)

(160)<smiles>COc1ccc(O)c(C[C@@H](C)O)c1O</smiles><smiles>C[C@@H]1Oc2cccc(O)c2[C@@H](O)[C@H]1O</smiles><smiles>O=Cc1c(O)cccc1CCCC(=O)O</smiles>

(166)

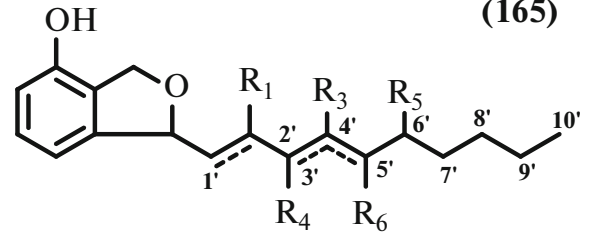<smiles>O=C1CCC(=C2CCC(=O)C3C[C@@H](O)c4c2ccc(O)c43)c2cccc(O)c21</smiles>

Cladosporol C

(169)
(171) $\Delta^{1^{\prime}-2^{\prime}}, \mathrm{R}_{1}=\mathrm{H}, \mathrm{R}_{2}=\mathrm{H}, \Delta^{3^{\prime}-4^{\prime}}, \mathrm{R}_{3}=\mathrm{H}, \mathrm{R}_{4}=\mathrm{H}, \mathrm{R}_{5}=\mathrm{OH}$

(172) $R_{1}=O H, R_{2}=O H, R_{3}=H, \Delta^{4^{\prime}-5^{\prime}}, R_{4}=H, R_{5}=H$

(173) $\mathrm{R}_{1}=\mathrm{OH}, \mathrm{R}_{2}=\mathrm{OH}, \mathrm{R}_{3}=\mathrm{H}, \mathrm{R}_{4}=\mathrm{OH}, \mathrm{R}_{5}=\mathrm{H}$

(174) $R_{1}=O H, R_{2}=O H, R_{3}=O H, R_{4}=O H, R_{5}=H$

Fig. 11. Phenols. (176)

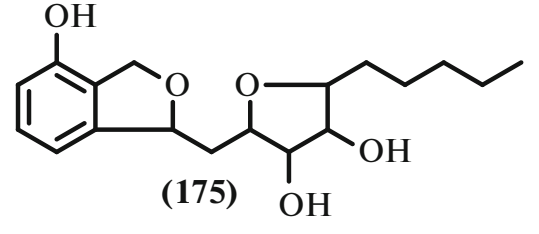$$
\text { . }
$$ 
<smiles>[R]c1c(O)cc(C)cc1Oc1c(C(=O)OC)cc(OC)c([R])c1O</smiles>

Pesteic acid

(177) $\mathrm{R}_{1}=\mathrm{H}, \mathrm{R}_{2}=\mathrm{CO}_{2} \mathrm{H}$ Pestalotether A

(178) $\mathrm{R}_{1}=\mathrm{Cl}, \mathrm{R}_{2}=\mathrm{CO}_{2} \mathrm{Me}$<smiles>[R2]CC(=C)c1c(Oc2c(OC)cc(O)cc2C(C)=O)cc(C)c([R3])c1O</smiles>

Asterric acid

(181) $\mathrm{R}_{1}=\mathrm{CH}_{3}, \mathrm{R}_{2}=\mathrm{H}, \mathrm{R}_{3}=\mathrm{H}$

Methyl asterrate

(182) $\mathrm{R}_{1}=\mathrm{CH}_{3}, \mathrm{R}_{2}=\mathrm{CH}_{3}, \mathrm{R}_{3}=\mathrm{H}$

(185) $\mathrm{R}_{1}=\mathrm{H}, \mathrm{R}_{2}=\mathrm{CH}_{3}, \mathrm{R}_{3}=\mathrm{H}$

Methyl 3-chloroasterric acid

(186) $\mathrm{R}_{1}=\mathrm{CH}_{3}, \mathrm{R}_{2}=\mathrm{H}, \mathrm{R}_{3}=\mathrm{Cl}$<smiles>Cc1cc(O)c(Oc2cc(C)cc(O)c2O)c(O)c1</smiles>

(180)<smiles>[R]c1c(O)cc(O)cc1Oc1cc(CCCCCCC)cc(O)c1[R]</smiles>

(183) $\mathrm{R}_{1}=\mathrm{R}_{2}=\mathrm{H}$

Aspermutarubrol

(184) $\mathrm{R}_{1}=\mathrm{R}_{2}=\mathrm{OH}$<smiles>CC(C)=CCc1c(C)cc(O)cc1Oc1cc(C)cc(O)c1</smiles>

Fig. 12. Diphenyl ethers.

mangrove endophyte Phyllosticta capitalensis [141] yielded the known polyketide, 3,4-dihydroxybenzoic acid (158), with antibiotic activity [174, 175].

In addition to phenols, related diphenyl ethers including several new compounds were isolated (Fig. 12). Cultivation of Penicillium sp., a marine sponge symbiont, allowed to isolate a new chlorinated diphenyl ether (179) [176].

The rest of diphenyl ethers were the known compounds. The study of the secondary metabolites of a fungus of the Pseudopestalotiopsis genus [102] afforded the known polyketides, i.e., pesteic acid (177) and pestalotether A (178) [177]. The seafloor fungus Penicillium chrysogenum MCCC 3A00292 [46] produces a series of antibiotic polyketides of different natures, i.e., 3,3'-dihydroxy-5,5'-dimethyldiphenyl ether (183) [178], aspermutarubrol (184) also known as violaceol I [179, 180], violaceol II (180) [180, 181], and asperde- min (133) [163]. Screening of marine micromycetes for the antibacterial activity gave the producer strain of six known metabolites [74] including polyketide, methyl asterrate (182) [182, 183]. Corresponding asterric acid (181) [184] was isolated from the culture liquid of micromycete of the Penicillium genus of mangrove origin [124]. A group of structurally related polyketides, consisting of a new (185) and three known polyketides, i.e., asterric acid (181) [184], methyl asterrate (182) [182, 183], and methyl-3-chloroasterric acid (186) [185]) was isolated from a mangrove fungus of the Pleosporales genus [186]. Only the known compound (186) among them exhibited significant activity.

New antibacterial diphenyl ether, diorcinol K (189) was isolated from marine micromycete Aspergillus sp. CUGB-F046 [187] along with the known analogs, diorcinols D and I $(\mathbf{1 8 7}, \mathbf{1 8 8})[188,189]$. 
<smiles>Cc1cc(O)c2c3c(c1=O)C(=O)C(=O)C3=C1O[C@H](C)C(C)(C)[C@@]1(O)C2=O</smiles>

Peniciphenalenin A (190)<smiles>[R]O[C@]1(C(=O)OC)OC(=O)C2=C3O[C@H](C)C(C)(C)[C@@]3(O)C(=O)c3c(C)cc(O)c1c32</smiles>

Peniciphenalenin B (191) $\mathrm{R}=\mathrm{H}$ Peniciphenalenin $\mathrm{C}$ (192) $\mathrm{R}=\mathrm{CH}_{3}$

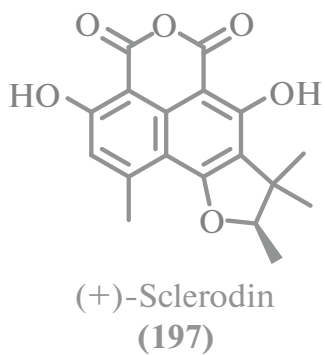<smiles>Cc1cc(O)c(C(=O)O)c2c1C1=C(C(=O)C2=O)C(C)(C)[C@@H](C)O1</smiles>

Peniciphenalenin D (193)

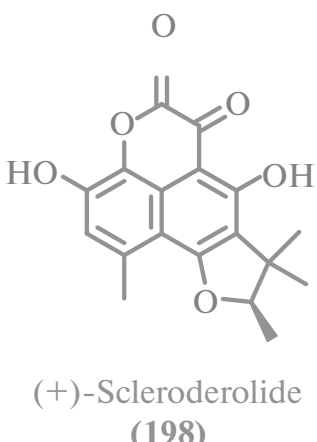<smiles>Cc1cc(O)c2c3c(c(O)c4c(c13)OC(C)C4(C)C)C(=O)O2</smiles>

Peniciphenalenin E (194)<smiles>C=c1oc2c(O)c3c(c4c(C)cc(O)c1c24)O[C@H](C)C3(C)C</smiles>

Peniciphenalenin F (195)

Fig. 13. Phenalenon derivatives.

Phenalenons are a fairly rare class of natural compounds [190]. When studying the marine fungus of the Penicillium genus [94], six undescribed phenalenon derivatives were isolated, i.e., peniciphenalenins $\mathrm{A}-\mathrm{F}$ (190-195) with no antibiotic activity (Fig. 13). At the same time, the known structural analogs, i.e., (+)-sclerodin (197) [191], (+)-scleroderolide (198) [192], and (+)-sclerodione (196) [193] exhibited a moderate cytotoxicity.

Spirocyclic metabolites with the grisan skeleton were found as secondary metabolites of extremophilic micromycetes (Fig. 14). The abovementioned producer of some benzophenones and anthraquinones [74] of marine origin along with the mentioned compounds synthesizes (-)-bis-dechlorogeodin (201) [194]. This previously described metabolite showed the antibacterial activity and moderate cytotoxicity. Cultivation of a marine sponge symbiont of the Penicillium genus [176] led to the isolation of the wellknown spirocyclic antifungal antibiotic griseofulvin (200) [131, 195].

Trypacidin (199), which was well-known for high cytotoxicity [196], was isolated from the strain of the Aspergillus genus [75].

Azaphilons are the common class of aromatic secondary fungal metabolites with a wide spectrum of biological activity [197]. Some representatives of this family including the halogenated analogs were isolated from extremophilic micromycetes (Fig. 14). According to the ASMAC strategy, the cultivation of this fungus was carried out in the presence of $5 \%$
$\mathrm{NaBr}$, which allowed to obtain another two brominated azaphilons, i.e., bromophilons A and B (210, 211). Stereomers (210) and (211) contain a unique for azaphilons structural motif, the aromatic side chain. Interestingly, bromophilon B (211) appeared to be noticeably more toxic than its isomer bromophilon A (210).

At the same time, several structurally close azaphilons were isolated from another fungus also of the marine origin of the Penicillium genus [198]. Newly isolated compounds, penicilazaphilons D and E $(205,206)$, did not exhibit a significant biological activity. Previously described $(+)$-sclerotiorin (207) [199], on the contrary, demonstrated a noticeable antiviral activity.

Another source of halogenated azaphilons was mangrove micromicete Diaporthe sp. SCSIO 41011 [200]. Six new oxidized chloroazaphilon derivatives, isochromophilons A-F (212-215, 202, 203), were isolated from the culture liquid of this strain, and isochromophilon D (215) showed the highest cytotoxicity. In addition to new compounds, several known analogs with antibiotic activity were isolated, i.e., epiisochromophilon II (204) [200, 201], epi-isochromophilon III (216) [201], and isochromophilon III (217) [201]. Known azaphiolons, pinophilin B (209) [202] and Sch 725680 (208) [202, 203], were isolated from mangrove producer Penicillium pinophilum SCAU037 [130].

Along with new compounds, known spirocyclic azaphilon mycotoxin H1 (218) [78, 204] was obtained 

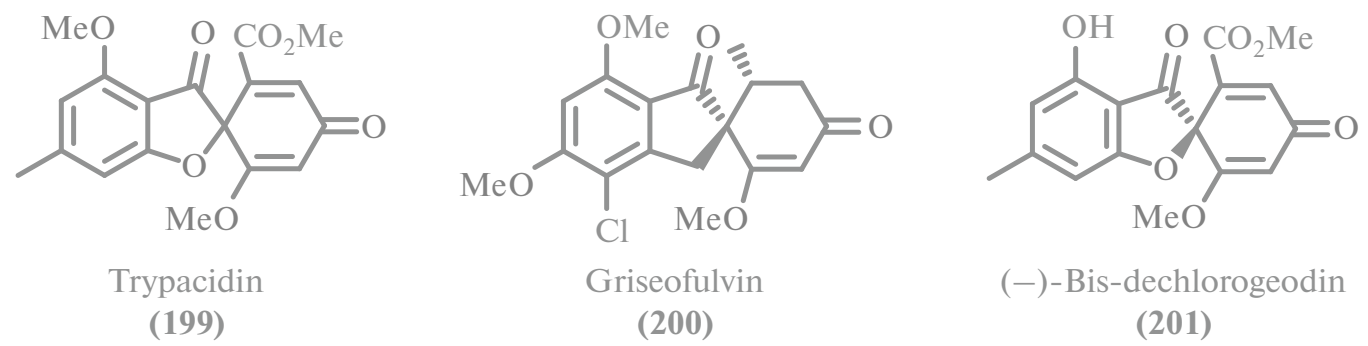

Fig. 14. Grisane-type compounds.

when studying a mangrove fungus of the Cladosporium genus [121]. Similar compounds, new polyketide xerucitrinin A (219), xerucitrinic acid A (220) [205], and penicitrinins A (221) and F (222) [206], were obtained from a fungus of marine origin [108].

Many polyketides that contained linear structure or long weakly branched chains including polyunsaturated ones (Fig. 16) were discovered among secondary metabolites of estremophilic micropicetes.

The systematic screening of marine microorganisms for the antibacterial activity revealed the strain of the Aspergillus genus [75], which produced sphingofungin I (239) and known polyketides including similar in structure sphingofungin D (240) [207]. Two new glycosilated alkylresorcins, resorcinosides A and $\mid B$ $(\mathbf{2 4 4}, \mathbf{2 4 5})$, were isolated from the Penicillium janthinellum fungus [208]. Compounds 244 and 245 are the first representatives of the structural family of resorcinols, which are glycosylated at the alkyl, and not the aromatic, part of the molecule. Substituted resorcinol B (245) showed pronounced cytotoxicity against several cell lines, whereas resorcinol A (244) was almost inactive $\left(\mathrm{GI}_{50}>50 \mu \mathrm{M}\right)$. New polyketidic metabolites, porosulfenols A-D $(\mathbf{2 3 4}, \mathbf{2 3 5}, \mathbf{2 3 7 a}, \mathbf{b})$ were obtained from the culture liquid of the Aspergillus porosus strain of marine origin [209]. Two latter compounds are in a dynamic equilibrium under ambient conditions (linear 237a and cyclic 237b forms). Psychrophilic micromycete isolated from Penicillium crustosum HDN153086 [40] in the Antarctic region appeared to produce a new polyene compound of unusual structure (238), which exhibited no biological activity. Another known linear polyketide, radiclonic acid (236) [210] was isolated from a seafloor fungus Penicillium chrysogenum MCCC 3A00292 [46].

Mangrove micromycete of the Fusarium solani genus H918 [211], along with antibiotic 1233B (241) [212], produces a series of previously undescribed polyketides, fusarisolins A-E (242, 243, 229-231). Despite the absence of a pronounced antibiotic activity, the molecules of these compounds contain the rare structural motifs, such as $\beta$ - and $\gamma$-lactone cycles in fusarisolins A (242) and B (243), respectively. Another mangrove endophyte Cladosporium sp. [116] became a source of linear polyketides $(232,233)$.
The combined cultivation of two micromycetes of extremophilic origin resulted in an unusual range of bioactive secondary metabolites. Four new alkylaromatic polyketides, penixylarines A-D (223-226), and two known [214] biosynthetic analogs, 5-(12hydroxyheptadecyl)resorcinol (227) and 5-(12-sulfoheptadecyl)resorcinol (228), were obtained from the combined culture of a deep-sea Antarctic fungus of Penicillium crustosum PRB-2 and a mangrove fungus Xylaria sp. HDN13-249 [213]. It should be noted that compounds (223) and (224) are biosyinthesized only when two producers are cocultured, while metabolites (225-228) can be produced by the Xylaria sp. Monoculture, although in significantly lower amounts.

The structures of other polyketide secondary metabolites are shown in Fig. 17. Some of these compounds have been described for the first time. In addition to new halogenated azaphilones, the cultivation of a marine sponge symbiont of the Penicillium genus [176] led to the isolation of the known mycotoxin, penicillic acid (249) [215-218]. A series of penicillic acid derivatives have also been isolated from marine micromycetes [42]. Cocultivation of this micromycete with Bacillus subtilis according to the OSMAC strategy resulted in obtaining two new derivatives of penicillic acid, i.e., ochraspergillic acids A and B $(253,254)$, in addition to penicillic acid itself (249).

A new macrolide, curvulaide A (257), with moderate antibacterial activity and cytotoxicity was isolated from a marine micromycete of the Curvularia sp. genus [219]. A new hybrid polyketide, cladodionen (252) was isolated from another fungus Cladosporium sp. OUCMDZ-1635 of marine origin [220]. Compound (252) is the equilibrium mixture of two geometric isomers (252a, 252b). Cladodionen (252) exhibited pronounced cytotoxic activity.

A thermophilic micromycete of the Penicillium genus [73] was isolated from hot springs in Saudi Arabia using bioactivity-based separation of a culture liquid extract, from which a new polyketide was obtained (246).

When studying a mangrove fungus of the Cladosporium genus [121], new active 1,1'-dioxin-2,2'-dipropionic acid (247) was obtained. 
<smiles>CC[C@H](C)/C=C(C)/C=C/C1=CC2=C(Cl)C(=O)[C@@]3(C)OC(=O)C(C(C)(OC)OC)=C3C2=CO1</smiles>

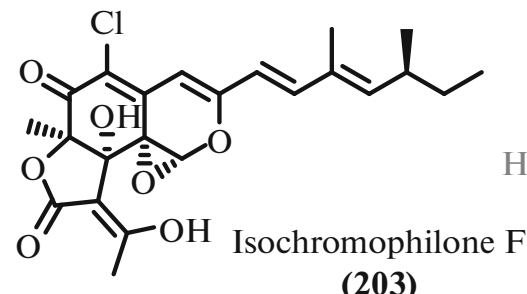

(203)<smiles>CC(=O)/C=C/C1=CC2=C(Cl)C(=O)[C@](C)(O)[C@H](O)[C@H]2CO1</smiles>

Penicilazaphilon D

(205)<smiles>C[C@H]1CCO[C@H]1[C@](C)(O)/C=C/C1=CC2=C(Cl)C(=O)[C@](C)(O)[C@@H](O)[C@]2(C)CO1</smiles>

Penicilazaphilon E

(206)<smiles>CC[C@H](C)/C=C(C)/C=C/C1=CC2=C(Cl)C(=O)[C@](C)(O)[C@H](CC(C)=O)C2=CO1</smiles>

(204)<smiles>[R]C=CC1=CC2=CC(=O)[C@H](O)[C@H](OC(=O)c3c(C)cc(O)cc3O)[C@@]2(C)CO1</smiles>

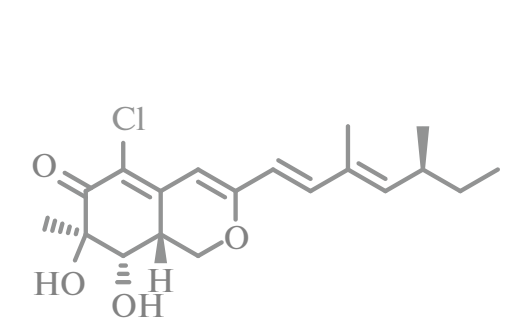

Epi-isochromophilone III (216) $8(S)$ Isochromophilone III (217) $8(R)$<smiles>CC[C@H](C)/C=C(C)/C=C/C1=CC2=C(Cl)C(=O)[C@](C)(OC(C)=O)C(=O)C2=CO1</smiles>

(207)
(210) $7(R)$
(211) $7(S)$<smiles>CC(=O)C1C2O[C@H](C)c3c(C)c(O)cc(c32)OC2CCCC21</smiles>

Citrinin H1 (218)<smiles>[R]OCCO</smiles><smiles>[R]c1c(O)c(C)c2c3c1O[C@](C)(CC)C[C@H](O[C@H](C)[C@@H]3C)[C@H]2C</smiles>

Xerucitrinin A (219) $\mathrm{R}=\mathrm{H}$

Xerucitrinic acid A (220) $\mathrm{R}=\mathrm{COOH}$<smiles></smiles>

Penicitrinons A, F

(221) $\mathrm{R}=\mathrm{H}$

(222) $\mathrm{R}=\mathrm{CH}_{3}$

Fig. 15. Azaphions.

Several well-known antibiotics of various structures were also isolated. In particular, trichodimerol (256) was isolated from the seafloor micromycete [59] [221]. A marine psychrotrophic fungus Aspergillus protuberus [222], which was selected by screening of some cultivated polyextremofilic microorganisms of the Barents Sea for the antibac- terial activity, was the source of the known antibacterial polyketide of the sorbicillonoids group, bisvertinolone (255) [223].

Seafloor fungus Penicillium chrysogenum MCCC 3A00292 [46] produces the known metabolites, decumbenones A and B (250, 251) [224], which were studied as cytotoxic agents. 


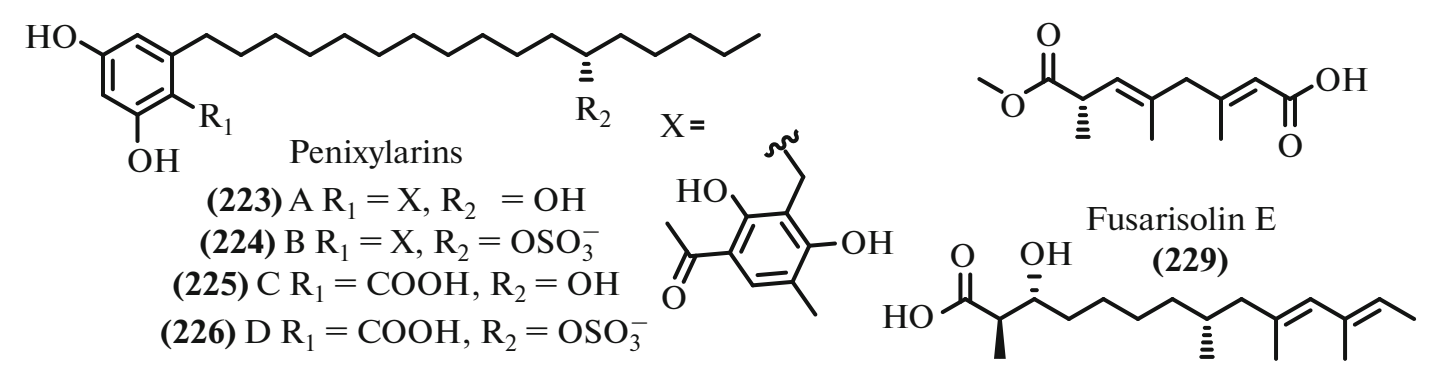

(227) $\mathrm{R}_{1}=\mathrm{H}, \mathrm{R}_{2}=\mathrm{OH}$

(228) $\mathrm{R}_{1}=\mathrm{H}, \mathrm{R} 2=\mathrm{OSO}_{3}^{-}$<smiles>COc1cc(C)cc(CC(C)=O)c1C(C)[C@@H](C)C(=O)[C@H](C)C(=O)/C(C)=C/C(C)=C/[C@H](C)[C@H](C)O</smiles>

(235) $4(R)$<smiles>C/C=C(/C)C=C(C)C[C@H](C)CCCC[C@H](O)[C@H](C)C(=O)O</smiles>

Fusarisolin C

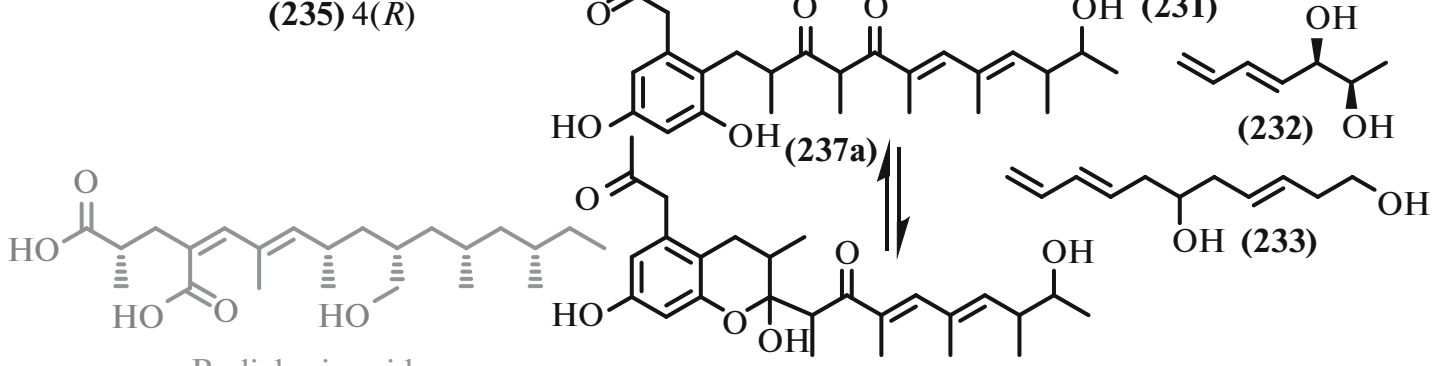

Radiclonic acid

(236)

(237b)<smiles>CCCCCC(O)CCCCCC/C=C/[C@@H](O)[C@H]1OC(=O)[C@H](NC(C)=O)[C@H]1O</smiles>

Sphingofungin I<smiles>CCCCCC(O)CCCCCC/C=C/[C@H](O)[C@@H](O)[C@H](O)[C@H](NC(C)=O)C(=O)O</smiles><smiles>CC(O)/C=C/C=C/C=C/C=C/C(=O)O</smiles><smiles>CC(=CC(=O)O)C=C(C)C=C(C)C[C@@H](C)CCCCC[C@@H](C)CCCCC1OC(=O)C1CO</smiles>

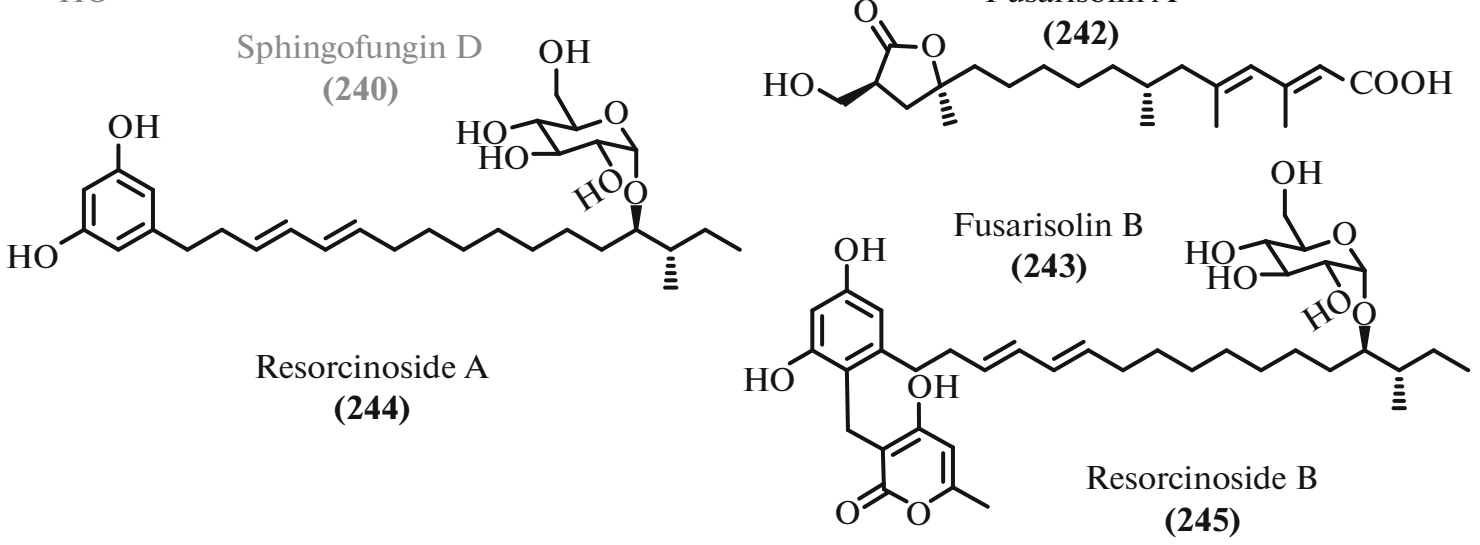

Fig. 16. Linear polyketides. 


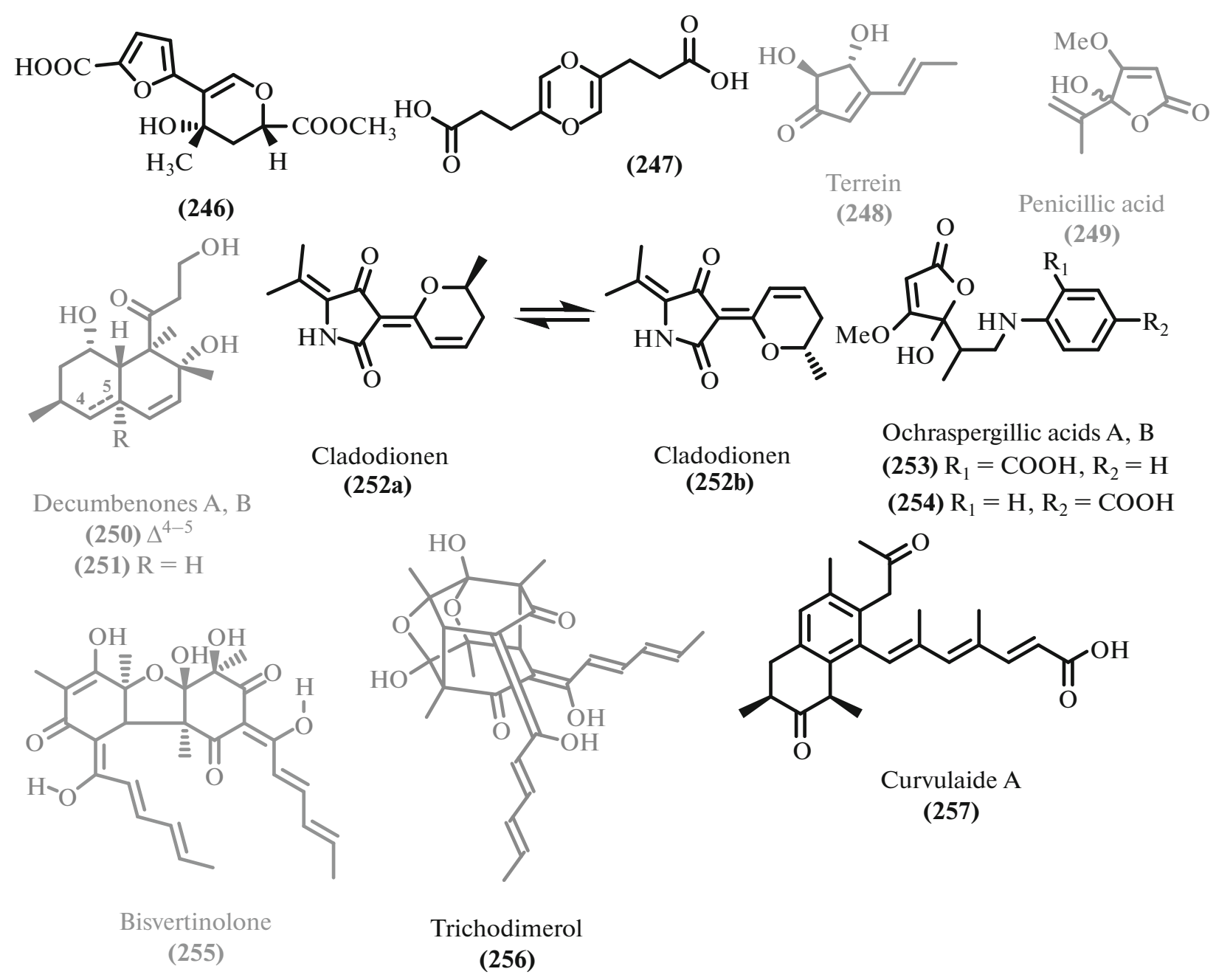

Fig. 17. Other polyketides.

The study of some isolates of marine micromycetes allowed to identify a producer of the well-known antibiotic terrein (248) [131, 225], with high productivity [226]. The selection of strains was based on the screening of the antimicrobial and cytotoxic activities of isolates.

\section{ALKALOIDS}

In the study of extremophilic micromycetes, more than seventy heterocyclic compounds were isolated, which can be assigned to alkaloids.

Penicipyrrodiether A (259), which was a new biosynthetic adduct of two known fungal metabolites, GKK1032B (258) [228] with a derivative of phenol A showed a pronounced antibacterial activity and cytotoxicity [229]. Interestingly, compound (258) was also isolated from another marine micromycete, Penicillium erubescens KUFA0220 [114, 230], and also turned out to be the most active antibiotic secondary metabolite among listed above. Seven new pyrrospirones $\mathrm{C}-\mathrm{I}$
(260-268) with a similar structure were isolated from marine fungus Penicillium sp. ZZ380 [78]. These pyrrospirones significantly differed in their biological activity. For example, only pyrrospirone G (266) showed the pronounced cytotoxicity against four cell lines. Pyrrospirones C (260), F (264), and I (267) exhibited the antibacterial activity including against resistant Gram-positive and Gram-negative bacteria.

Extremophilic micromycetes became the source of some alkaloids containing the indole core and its derivatives (Figs. 19, 20).

Indole-diterpene-type alkaloids (Fig. 19) were isolated from micromycetes of various origins. A marine fungus of the Aspergillus genus [231] became the source of four new indole diterpene-type alkaloids, asperindoles A-D (279-282). Only one of them, compound (279), showed cytotoxic activity. When studying the Tolypocladium sp. XL115 [232] strain isolated from the soil of a coal mine, ten new prenylated diterpene alkaloids, called tolipocladins A-J (269- 

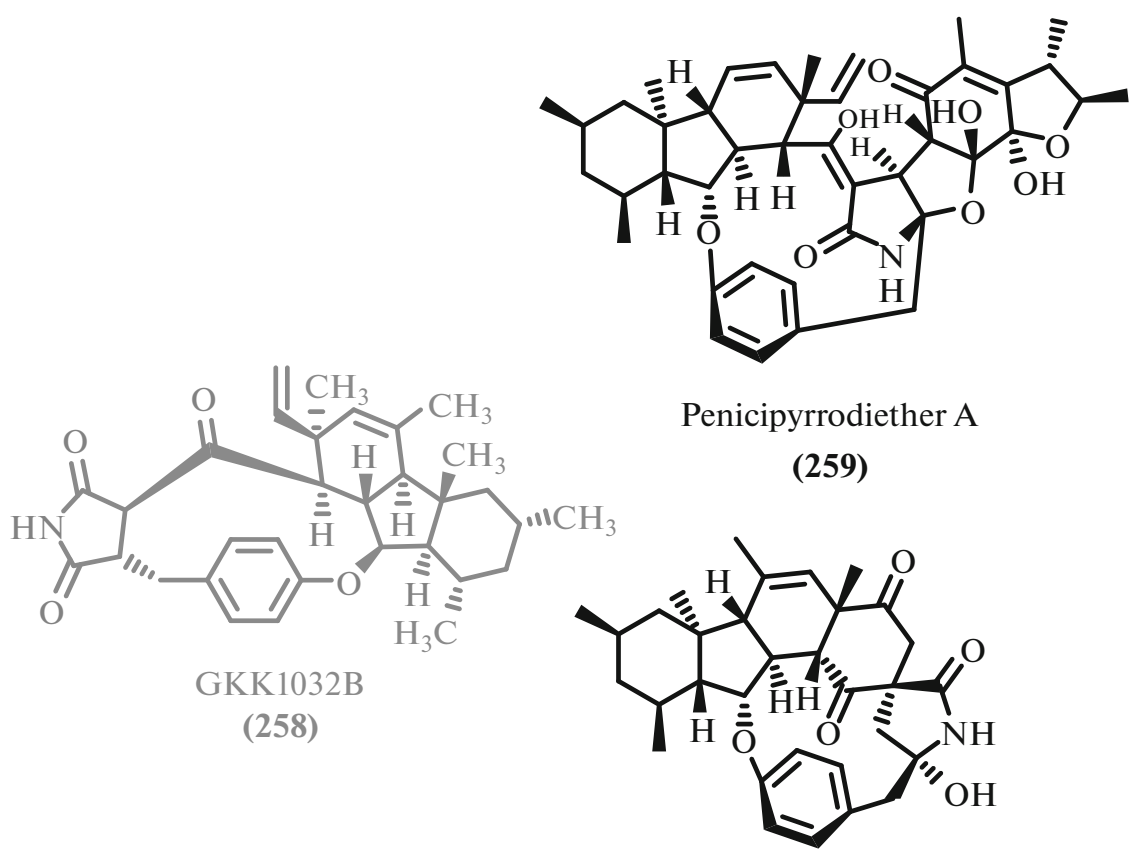

Pyrrospiron $\mathrm{G}$

(266)

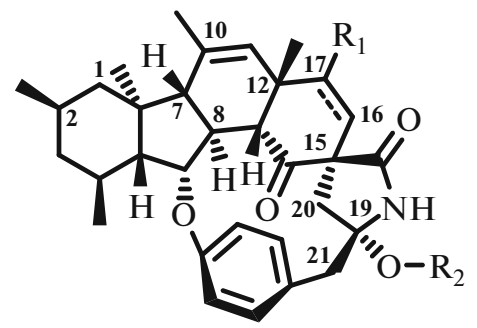

Pyrrospiron C, D (260) $17(R) \mathrm{R}_{1}=\mathrm{OH}, \mathrm{R}_{2}=\mathrm{CH}_{3}$ (261) $17(S) \mathrm{R}_{1}=\mathrm{OH}, \mathrm{R}_{2}=\mathrm{CH}_{3}$

Pyrrospiron E, F

(262) $17(R) \mathrm{R}_{1}=\mathrm{OH}, \mathrm{R}_{2}=\mathrm{H}$

(263) $17(R) \mathrm{R}_{1}=$ OAc, $\mathrm{R}_{2}=\mathrm{H}$

(264) $17(S) \mathrm{R}_{1}=\mathrm{OH}, \mathrm{R}_{2}=\mathrm{H}$

(265) $17(S) \mathrm{R}_{1}=$ OAc, $\mathrm{R}_{2}=\mathrm{H}$

Pyrrospiron $\mathrm{H}, \mathrm{I}$

(267) $\mathrm{R}_{1}=\mathrm{H}, \mathrm{R}_{2}=\mathrm{H}$

(268) $\Delta^{16-17}, \mathrm{R}_{1}=\mathrm{H}, \mathrm{R}_{2}=\mathrm{H}$

Fig. 18. GKK1032B, penicipyrrodiether A, and pyrrospirones.

278), were obtained. Compounds $(269,276)$ showed significant antimicrobial activity.

The structures of other indole alkaloids are summarized in Fig. 20. Two new alkaloids, i.e., 4-episeco-shornephinic acid A (300) and its methyl ester (299), along with the well-known biosynthetically bound analog, shornephine A (303) [233], that contained a rare diketomorpholine motif were isolated from the endophyte of seaweed [234].

Previously undescribed alkaloids, candidusine D (296) and preussin C (329), were isolated from marine fungus Aspergillus candidus KUFA0062 [79] along with related compounds. Only petromurine C (295) [235] and preussin (328) [236] among the latter exhibited significant activity. The other compounds, i.e., asterquinol D (296) and cumbicins A, B, and D (327, 298, 304) [235], showed weak cytotoxicity.

Deep-sea micromycete Aspergillus fumigatus SCSIO 41012 [237] turned out to be the source of many antibiotic secondary metabolites. Two new alkaloids, fumigatosids E (283) and F (284), and previously described their structural analogs, fumiquinazolines C (285) and G (286) [238], and epi-aszonalenin A (288) [239] were isolated.

An interesting example of the successful application of cocultivation of two marine micromycetes (in addition to the above mentioned method [213]) to diversify the spectrum of secondary metabolites of micromycetes is the isolation of five new indole alkaloids, i.e., 17-hydroxynotoamide D (287), 17- $O$-ethy- lonotoamide $\mathrm{M}$ (292), 10- $O$-acetylsclerotoamide (289), 10- $O$-ethylsclerotoamide (290), and 10 - $O$-ethylnotoamide R (293) [240].

The study of the relationship between the structure and cytotoxic activity of new indole alkaloid misszrtine A (291) isolated from marine Aspergillus sp. SCSIO XWS03F03 [241] showed a significant effect of the functionalization of indole nitrogen on biological properties.

Original indole diterpenes, penicillindoles $\mathrm{A}-\mathrm{C}$ (305-307), were isolated from mangrove fungus Eupenicillium sp. HJ002 [242]. Penicillindoles A (305) and $\mathrm{B}(\mathbf{3 0 6})$ that contained no additional cycle showed a noticeable cytotoxic activity. Compound (305) that contained the $\mathrm{OH}$ group at position 19 appeared to be more active than its carbonyl analog (306).

Two new oxyindole epimeric diterpenes, anthcolorins $\mathrm{G}$ (301) and $\mathrm{H} \mathrm{(302)}$, were significantly different in their biological activity. Only epimer (302) showed moderate activity.

Some isolated alkaloids can be considered as derivatives of maleimide and pyrrolidone (Fig. 21). Three new macrocyclic alkaloids, ascomylactams A-C (308-310) were isolated from mangrove endophyte Didymella sp. CYSK-4 [244], along with the known analogs, phomapyrrolidones $\mathrm{A}$ and $\mathrm{C}(\mathbf{3 1 1}, \mathbf{3 1 2})$ [245], structure of which was refined. Two new stereoisomeric alkaloids, cladosporitins A (313) and B (314), were isolated from the culture fluid of the mangrove micromycete Cladosporium sp. HNWSW-1 


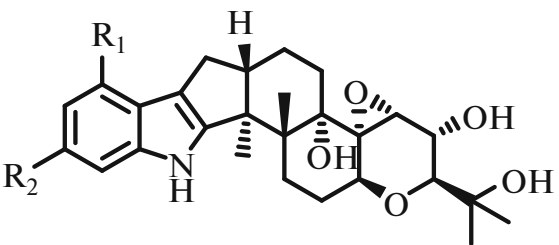

Tolypocladin A (269) $R_{1}=A, R_{2}=H$

Tolypocladin B, C (270) $\mathrm{R}_{1}=\mathrm{H}, \mathrm{R}_{2}=\mathrm{B}$ (271) $\mathrm{R}_{1}=\mathrm{H}, \mathrm{R}_{2}=\mathrm{C}$

Tolypocladin D (272) $R_{1}=H, R_{2}=D$

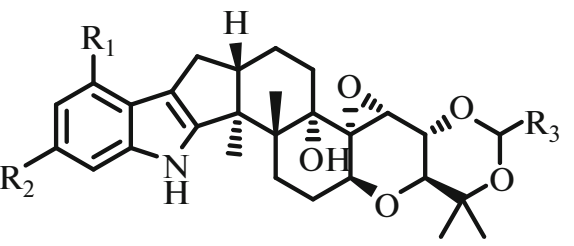

Tolypocladin $\mathrm{H}$ (276) $R_{1}=A, R_{2}=H, R_{3}=F$

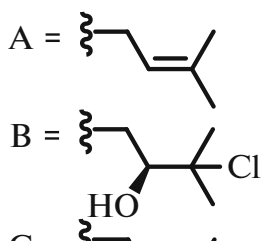<smiles>C=C[C@@H](O)C(C)(C)OC</smiles><smiles>CC(C)(C)C[C@@H]1[C@H](C(F)(F)F)[C@@H](O)C(C)(O)CC1(C)C</smiles><smiles>CC(C)=C[Te][Te](=O)(F)F</smiles>

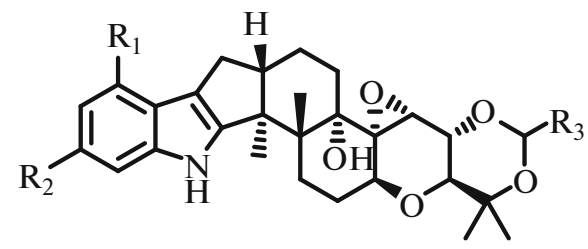

Tolypocladin E, F

(273) $\mathrm{R}_{1}=\mathrm{B}, \mathrm{R}_{2}=\mathrm{H}, \mathrm{R}_{3}=\mathrm{F}$

(274) $R_{1}=E, R_{2}=H, R_{3}=F$

Tolypocladin $\mathrm{G}$

(275) $R_{1}=H, R_{2}=E, R_{3}=F$

Tolypocladin I

(277) $R_{1}=E, R_{2}=H, R_{3}=G$

Tolypocladin $\mathbf{J}$
Asperindoles A, B

(279) $\mathrm{R}=\mathrm{Cl}$

(280) $\mathrm{R}=\mathrm{H}$

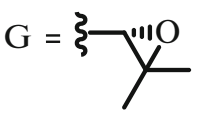

Fig. 19. Tolipocladins and asperindoles.

[246], along with a known analog, talaroconvolutin A (315) [247]. Interestingly, despite the same planar structure, only cladosporitin B (314) showed noticeable cytotoxicity.

Some isolated alkaloids can be assigned to pyridone derivatives (Fig. 22). Seafloor micromycete Arthrinium sp. UJNMF0008 [248] produces a series of eight alkaloids, artpirons D-K (316-323), that contain unusual structural motifs, and a known analog, apiosporamide (324) [249].

Systematic screening of marine micromycetes for a wide range of antibacterial activity revealed a strain of the Aspergillus genus [75], which produced a new alkaloid, chaetominine A (326), along with known chaetominine (325) [250].

Thermophilic micromycete [126] isolated from hydrothermal vents was found to produce several antibiotic compounds, the most active of which were the known alkaloids, leporin A (327) (previously described as an insecticide [251]) and chaetominine (325) [250].
Known alkaloid penicidone C (330) [252] was isolated from soil mangrove producer Penicillium pinophilum SCAU037 [130]. When studying a mangrove fungus of the Cladosporium genus [121], moderately active new alkaloid 2-acetoxymethyl-3,5,6-trimethyl pyrazine (331) was isolated along with polyketides. New alkaloid acremolin C (332) with a moderate antibacterial activity was isolated from Antarctic soil fungus Aspergillus sydowii SP-1 [44]. The authors of [253] suggest that this compound may be identical to the previously described acremolin B, which contains the isopropyl group at position 2', not 1'. Another pyrazine alkaloid flavacol (333) was re-isolated from mangrove Aspergillus sp. SCSIO41211 [254].

\section{TERPENOIDS, STEROIDS, AND RELATED COMPOUNDS}

A significant part of the secondary metabolites of extremophilic micromycetes can be assigned to terpenoids, steroids, or related compounds. Sesquiterpenes and sesquiterpenoids (334-393) (Figs. 24, 25), 


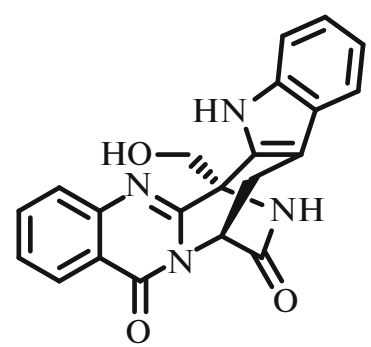

Fumigatosid E (283)<smiles>C=CC(C)(C)[C@]12Nc3c(ccc4c3C=CC(C)(C)O4)[C@]1(O)C[C@H]1C(=O)N3CCC[C@]3(O)C(=O)[C@@H]12</smiles>

17-Hydroxynotoamide D (287)

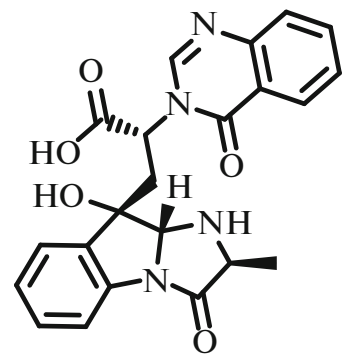

Fumigatosid $\mathrm{F}$

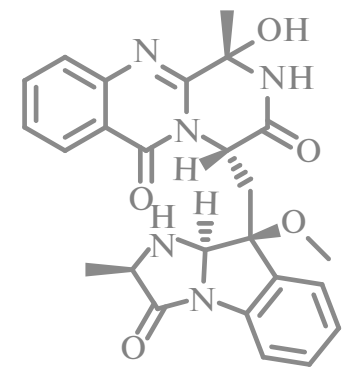

Fumiquinazoline $\mathrm{C}$

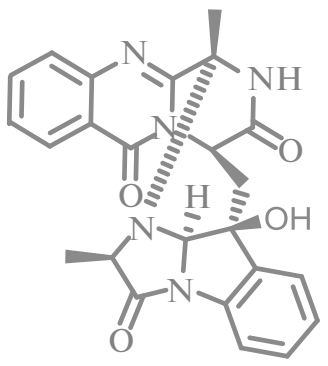

Fumiquinazoline $\mathrm{G}$<smiles>COC(=O)[C@H](Cc1cn(CC=C(C)C)c2ccccc12)NC(=O)[C@H](O)Cc1ccccc1</smiles>

Misszrtine A

(291)

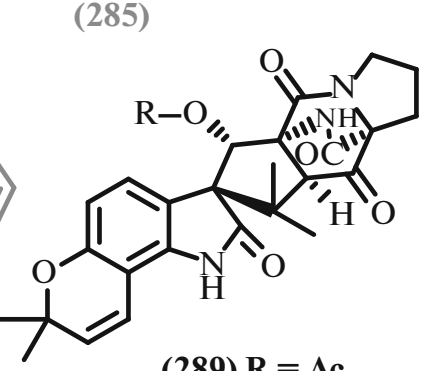

(289) $\mathrm{R}=\mathrm{Ac}$ (288)<smiles>C=CC(C)(C)[C@]12C[C@@H]3C(=O)Nc4ccccc4C(=O)N3C1N(C(C)=O)c1ccccc12</smiles>

Epi-aszonalenin A<smiles>C=CC(C)(C)[C@]1(CC2NC(=O)[C@@]3(OCC)CCCN3C2=O)C(=O)Nc2c1ccc1c2C=CC(C)(C)O1</smiles>

17-O-ethylnotoamide M

(292)<smiles>[R7]OC(=O)/C(=C/C[C@@]1(C(C)(C)C=C)C(=O)Nc2c(O)cccc21)NC(=O)[C@@H](O)Cc1ccccc1</smiles>

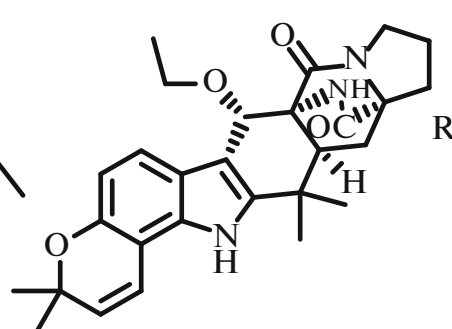

10-O-ethylnotoamide R<smiles>[R]CCCCCCC</smiles>

(293)<smiles>CC1(C)CNc2ccccc21</smiles><smiles>C=C1O[C@H](Cc2ccccc2)C(=O)N2[C@@H]1C[C@@]1(O)c3cccc(CC)c3N[C@@]21C(C)(C)C</smiles>

(294) $R_{1}=R_{2}=H$

(295) $R_{1}=O H, R_{2}=H$

(296) $\mathrm{R}_{1}=\mathrm{R}_{2}=\mathrm{OMe}$

Cumbicin A, B

(297) $\mathrm{R}_{1}=\mathrm{R}_{2}=\mathrm{OH}$

(298) $\mathrm{R}_{1}=\mathrm{OMe}, \mathrm{R}_{2}=\mathrm{H}$<smiles>C1CCCCC1</smiles><smiles>C=C1[C@H](C)CC[C@H]2[C@]1(C)CC[C@@H](O)[C@]2(C)CCC=C(C)C</smiles>

Shornephine A

(303)<smiles>C=C1C(=C)C(CC)=C(c2c[nH]c3ccccc23)C(=O)C1=CC=CC</smiles>

\begin{abstract}
Anthcolorin G (301) $3(R)$

Anthcolorin $\mathrm{H}$ (302) $3(S)$
\end{abstract}

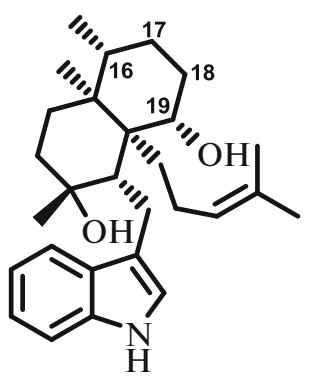

Penicilindole A (305)
Penicilindole B

(306)

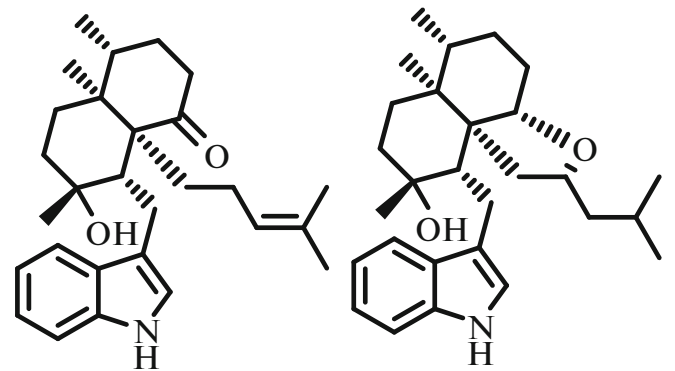

Penicilindole $\mathrm{C}$ (307)

Fig. 20. Indoles and their derivatives. 


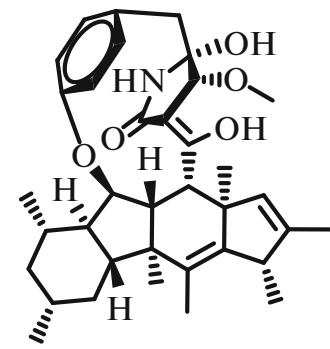

Ascomylactam A (308)

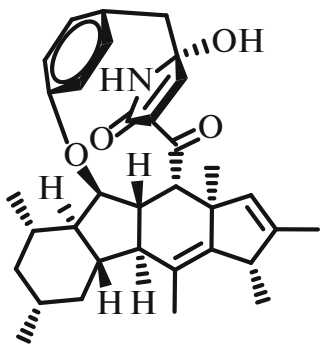

Ascomylactam B (309)

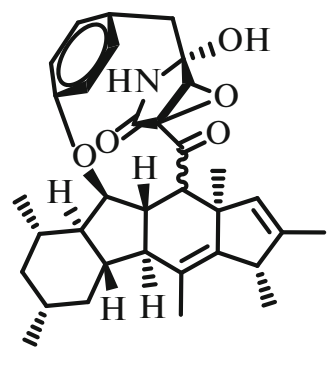

Ascomylactam C (310) $16(R)$

Phomapyrrolidone A (311) $16(S)$

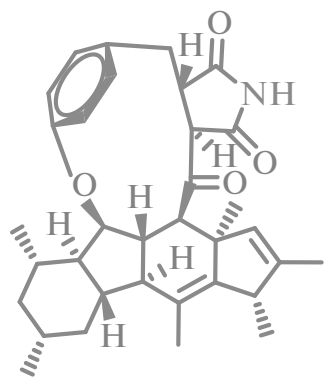

Phomapyrrolidone C (312)

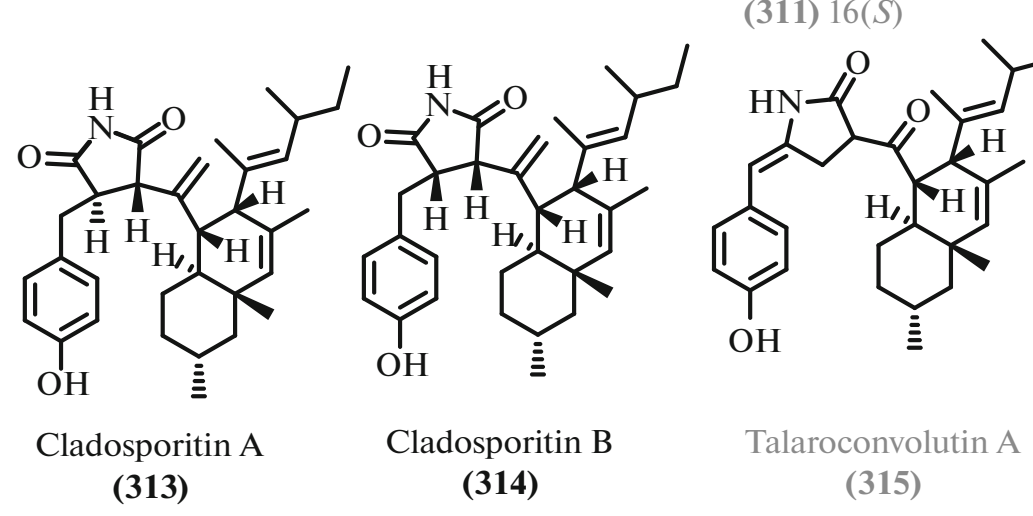

Fig. 21. Pyrrolidone and maleimide derivatives.

diterpenes and diterpenoids (394-420) (Fig. 26), steroids, sterols, and their derivatives $(\mathbf{4 2 1}-\mathbf{4 5 7})$ were isolated (Figs. 27, 28).

Many previously undescribed sesquiterpenoids were isolated, and many of them did not show biological activity. For example, three new inactive sesquiterpenes of pestalotiopsin I-K (334-336) were isolated from Pseudopestalotiopsis sp. PSU-AMF45 [102]. A new sesquiterpene (337) that did not show the antibiotic activity was isolated from a mangrove fungus of the Pleosporales genus [186].

Six new sesquiterpenes (338-342) and four known sesquiterpenes (343-346) [256], which belonged to the ophioboline family, were isolated from marine endophyte Aspergillus flocculosus [255]. All the isolated compounds showed strong cytotoxic activity.

Nitrobenzoic sesquiterpenes are rarely found among natural compounds. Three new nitrobenzoylsesquiterpenes, insulicolides $\mathrm{B}$ and $\mathrm{C}$ and 14- $O$ acetylnsulicolide $\mathrm{A}(\mathbf{3 5 1}, \mathbf{3 5 2}, \mathbf{3 4 9})$, and known active analogs $(\mathbf{3 5 0}, \mathbf{3 5 3}, \mathbf{3 5 4})[258,259]$ were isolated from marine fungus Aspergillus ochraceus Jcma1F17 [257].

Three new sesquiterpenes of the eremophylane type were isolated from marine fungus Cochliobolus lunatus SCSIO41401 [76]. The isolated compounds belong to the structural family of dendryphiellins; they were called dendryphiellins $\mathrm{H}-\mathrm{J}(\mathbf{3 5 5}, \mathbf{3 4 7}, \mathbf{3 4 8})$. Two of these compounds $(347,348)$ exhibited high cytotoxic activity, whereas compound (355) was not active, which indicated the critical importance of the side chain of dendryphiellins for the implementation of their biological properties.

The spectrum of secondary metabolites of mangrove endophyte Cytospora sp. was studied [260]. The authors of [261] isolated bicyclic sesquiterpene, seiricardine D (356), with no biological activity and determined the structure of eight known metabolites. The antibacterial activity was shown for $(22 E, 24 R)-5,8$ epidioxy- $5 \alpha, 8 \alpha$-ergosta-6,9(11),22-trien-3 $\beta$-ol (357).

Two known terpenes with moderate antibacterial activity, (7S)-(+)-hydroxydonic acid (358) [262] and $(7 S, 11 S)-(+)-12$-hydroxydonic acid (359) [263], were isolated from Antarctic soil fungus Aspergillus sydowii SP-1 [44].

Mangrove endophyte Aspergillus sp. xy02 [264] became the source of seven new phenolic bisbolanic sesquiterpenes $(\mathbf{3 6 0}-\mathbf{3 6 4}, \mathbf{3 6 6}, 367)$, which showed weak antibacterial activity. Interestingly, epimers (361) and (362) were found to be completely inactive [264]. Terpene (367) that contained the $E$-double bond instead of the $Z$-double bond as in (366) was also inactive. Some previously described analogs, i.e., 


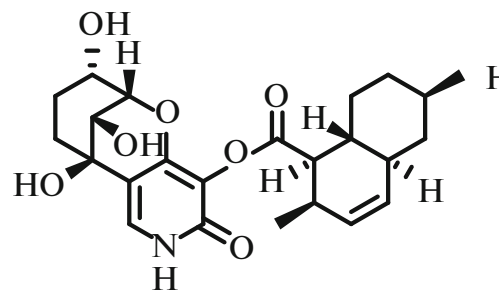

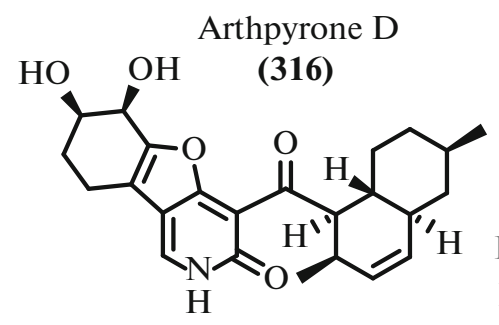

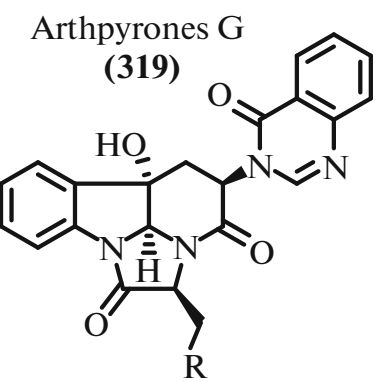

Chaetominine (325) $\mathrm{R}=\mathrm{H}$

Chaetominine A (326) $\mathrm{R}=\mathrm{OH}$

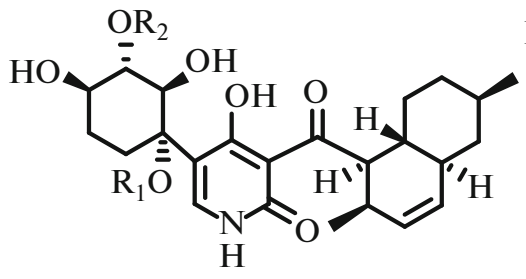<smiles>C[C@H]1CC[C@H]2C=C[C@@H](O)[C@@H]3C(=O)c4c(c[nH]c(=O)c43)O[C@]2(O)[C@H]1O</smiles>

Arthpyrones E, H, I

(317) $\mathrm{R}_{1}=\mathrm{H}, \mathrm{R}_{2}=\mathrm{H}$

(320) $\mathrm{R}_{1}=\mathrm{H}, \mathrm{R}_{2}=\mathrm{Me}$

(321) $\mathrm{R}_{1}=\mathrm{Me}, \mathrm{R}_{2}=\mathrm{H}$

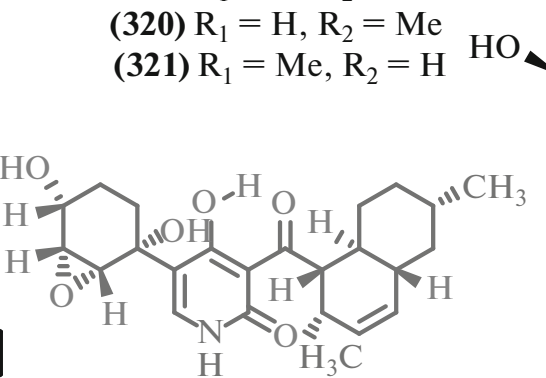

Arthpyrones F

(318)

Arthpyrones J, K (322) $\mathrm{R}_{1}=\mathrm{OH}, \mathrm{R}_{2}=\mathrm{H}$ (323) $\mathrm{R}_{1}=\mathrm{H}, \mathrm{R}_{2}=\mathrm{OH}$

Apiosporamide (324)

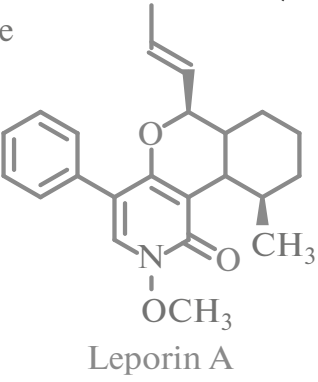

(327)

Fig. 22. Pyridone derivatives.

engiodontiumon I (365) [265], hydroxysydonic acid (359) $[262,263]$, and (-)-(7S)-10-hydroxysydonic acid (368) [263, 264], were active.

Four new sesquiterpenoids, penicieudesmols A-D (369-372), were isolated from a mangrove endophyte of the Penicillium genus; however only one of them showed weak cytotoxicity [266].

Six sesquiterpenoids, piltunins A-F (373-378) were isolated from marine fungus Penicillium piltunense KMM 4668 [151] along with the known structural analog, penigrisacide D (385) [267].

In addition to diterpene alkaloids, nine new sesquiterpenoids (379-384, 386-388) were isolated from the culture liquid of mangrove endophyte Aspergillus versicolor [243]. Two of these compounds, i.e., 7-deoxy-7,14-didehydro-12-acetoxy-sydonic acid (386) and its isomer 7-deoxy-7,8-didehydro-12-acetoxysydonic acid (387), showed a weak cytotoxic activity.

A new abscisic acid analog, cladosacid (393), was isolated from fungus Cladosporium sp. OUCMDZ1635 of marine origin [220].
Among the secondary metabolites of micromycetes, diterpenes and diterpenoids were no less common than sesquiterpenes and their derivatives (Fig. 26). In some cases, diterpenoids were isolated along with sesquiterpene metabolites. A new sesquiterpenoid 9,10-diolhinokic acid (389) and the diterpenoid roussoellol $\mathrm{C}(\mathbf{4 0 4})$ and the well-known dankasterone (405) [268, 269] were found among secondary metabolites of marine fungus Talaromyces purpurogenus [54]. The cultivation of marine fungus Trichoderma erinaceum F1-1 [270] led to the isolation of four new (close to known) terpenoids of different structures, diterpenoid (420) and sesquiterpenoids (390-392), which did not show biological activity [303].

Three new diterpenoids of the dolabellanic type, stachatranones A-C (394-396), and three new atranones Q-S (397-399) were isolated from marine fungus Stachybotrys chartarum [271]. New atranones have a significantly new structure. Atranone Q (397) is the first $\mathrm{C}_{23}$ atranone that contains the propan-2-one motif attached to the dolabellane core through the $\mathrm{C}-$ 


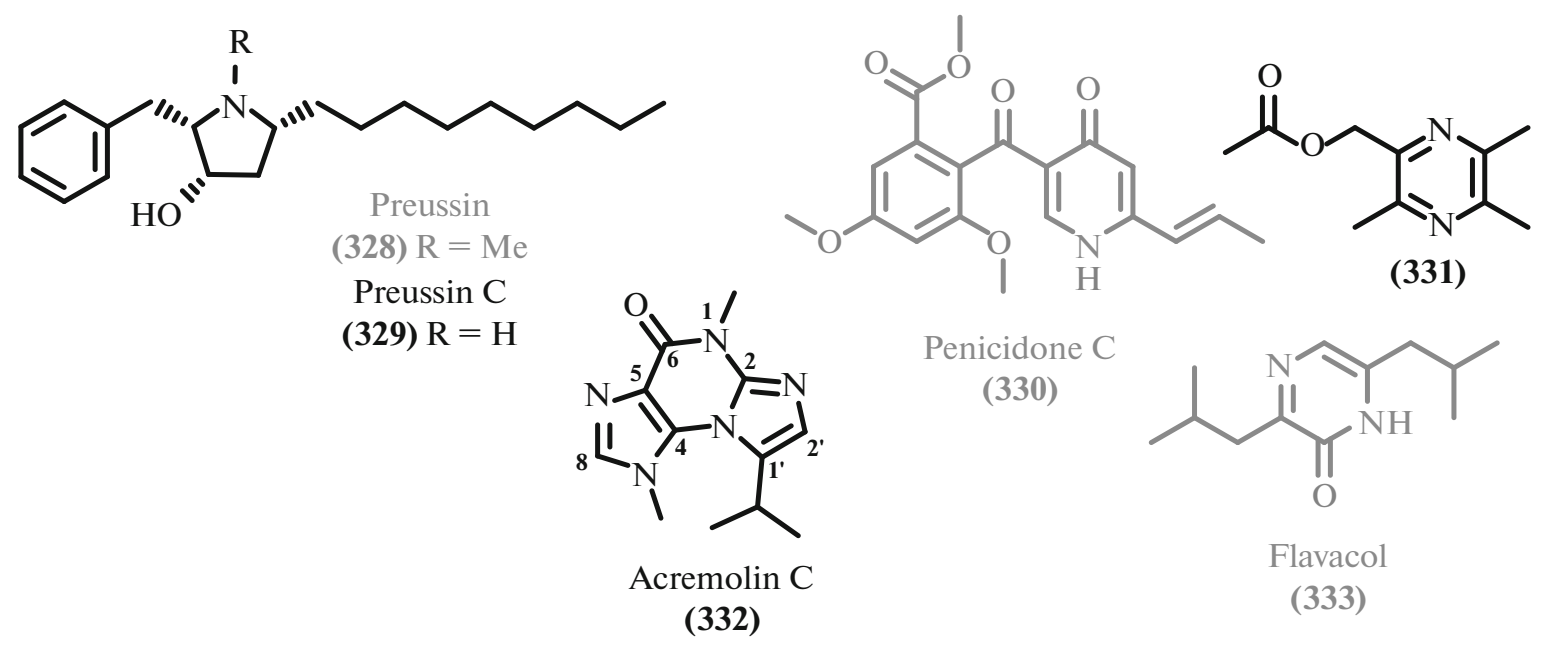

Fig. 23. Other alkaloids.

$\mathrm{C}$ bond. Antranone $\mathrm{R}$ (398) is the first representative of $\mathrm{C}_{24}$ antranones that contains the condensed 2methyltetrahydrofuran-3-carboxylate cycle at the C5-C-6 position (Fig. 26; the noted fragments are highlighted in red).

Separation of toxic fractions of the culture liquid extract of the marine Aspergillus porosus strain [209] led to the release of the known phytotoxin, sphaeropsidin A (401) [272, 273], which recently attracted interest as a cytotoxic agent [274]. Interestingly, the second isolated analog, which is very similar in structure to the known aspergiloid E (400) [273], has a significantly lower activity.

Fungus Penicillium sp. TJ403-1 isolated from the marine coral [51] turned out to be a producer of several cytotoxic spiroditerpenoids of the brevione family. New brevione $\mathrm{O}$ (403) did not show valuable pharmacological properties, while the well-known brevione I (402) [275] was active against several tumor cell lines.

Seven new diterpenoids of the pimarane type, libertellenones $\mathrm{O}-\mathrm{S}(\mathbf{4 1 0 - 4 1 4 )}$ and eutypeellenones $\mathrm{A}$ and $B(\mathbf{4 0 6}, \mathbf{4 0 7})$, were isolated from the culture liquid of soil Arctic fungus Eutypella sp. D-1 [276] along with known libertellenones $\mathrm{H}$ and $\mathbf{J}(\mathbf{4 0 8}, \mathbf{4 0 9})$ [277].

All isolated compounds showed cytotoxic activity. The high diversity of structures allowed to establish some structure-activity relationships. For example, methoxylation at the $\mathrm{C}-10$ position is favorable for the cytotoxic action, while the reversal of the $\mathrm{C}-13$ configuration and hydroxylation at the $\mathrm{C}-14$ position reduce the activity.

Five new 20-nor-isopimarane diterpenoids including the set of four diastereomers, aspewentines $\mathrm{I}-\mathrm{L}$ (415-418), and the methylated derivative, aspewentine M (419), were isolated from marine fungus Aspergillus wentii SD-310 [278].

Another structural family of isolated secondary metabolites is steroids and related compounds. New sterols are rare among secondary metabolites of microorganisms, which makes it interesting to detect the new sterol, 3,7-diketo-cephalosporin P1 (421), in the culture liquid of the deep-sea Aspergillus fumigatus SCSIO 41012 [237]. In addition to the new sterol, two known compounds of this class were isolated, i.e., helvolic acid (424) [279, 280] and 22-acetylisocyclocitrinol A (435) [281]. Interestingly, new sterol (421) showed a noticeable specific activity against Acinetobacter baumannii. Helvolic acid (424) and its seven new derivatives $(\mathbf{4 2 2}, \mathbf{4 2 3}, \mathbf{4 2 5}-\mathbf{4 3 0})$ were isolated from marine fungus Aspergillus fumigatus HNMF0047 [282]. Compounds (422-424) exhibited maximal antibiotic activity.

Seaweed endophyte Aspergillus alabamensis EN547 [234] was the source of a new steroid of ergostane type, 28-acetoxy-12 $\beta, 15 \alpha, 25$-trihydroxyergosta4,6,8(14),22-tetraen-3-one (431), which demonstrated a weak aqntimicrobial activity.

20-Acetoxy-16 $\alpha$-methylpreg-17 $\alpha, 19$-dihydroxy-(9,11)epoxy-4-en-3,18-dione (433) and previously described toxic terpenoid, terretonin (439) [283], were isolated from the marine Penicillium citrinum SCSIO 41017 strain [108].

Two new terpenoids, asperterpens $\mathrm{N}$ (436) and $\mathrm{O}$ (437) were isolated from seaweed endophyte Aspergillus terreus EN-539 [284] along with related known compounds, terretonins A [285] and G (440) [286], the stereoconfiguration of which was clarified. As before [286], terretonin (444), demonstrated weak antibacterial activity [284]. Later, new terpenoid, terretonin $\mathrm{O}$ (442), was isolated from thermophilic Aspergillus terreus TM8 and marine Aspergillus terreus LGO13 [287]. Presumably, compound (442) is a biosynthetic precursor of the recently isolated terretonins M (441) [288] and N (443) [289]. These compounds are oxidized at the $\mathrm{C}-11$ position, which is not typical 


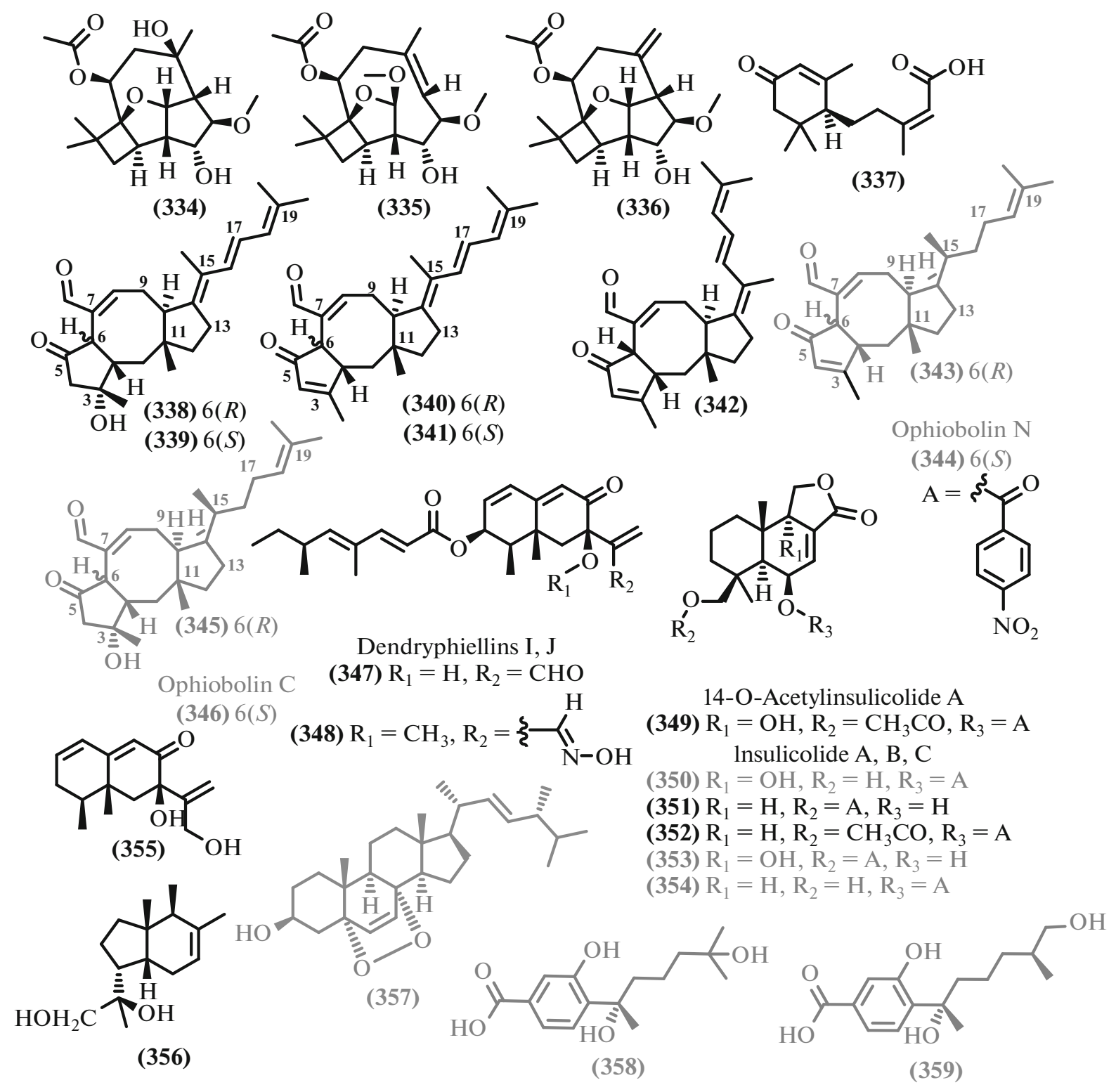

Fig. 24. Sesquiterpenes and sesquiterpenoids.

for this class of compounds but their antibacterial activity is very low.

A new ergostan, sarocladione (444), is the first steroid that contains the unique 5,10:8,9-diketo motif. This compound was isolated from deep-sea fungus Sarocladium kiliense [290]. In addition to sarocladione (444), twenty known compounds were isolated, and some of them showed the antibiotic properties. A high cytotoxic activity was detected for $\beta$-sitostenone (438) [291] and 4,6-dihydroxyeudesmane (456) [292]. 22E$7 \alpha$-Methoxy- $5 \alpha, 6 \alpha$-epoxyergosta- $8(14), 22$-dien-3 $\beta$ ol (455) [293], diosgenin (457) [294], and gramisterol (432) [291] demonstrated a moderate activity. Among the known sterols, we should also note $\beta$-sitosterol (454) [295] isolated from seafloor Aspergillus sydowii
C1-S01-A7 [77]. Mangrove Aspergillus sp. SCSIO41211 [254] was the source of new steroid (434) that did not show activity.

Active secondary metabolites of deep-sea micromycete Penicillium granulatum MC 3 A00 475 [47] include several compounds of the steroid nature, i.e., isonuatigenin I (453) [296], penicysteroid A (446) [297], and anicequol (445) [298].

Five new ergostans, penicysteroids D-H (448452) were obtained from the deep-sea strain of Penicillium granulatum MCCC 3A00475 [299] along with fourteen known steroids, some of which exhibited the cytotoxic activity (primarily penicysteroids A and C $(446,447)[297,300]$. 
<smiles>C=C(CCCC(C)CO)c1ccc(C(=O)O)cc1O</smiles>

(360) $7(R)$

(361) $7(S)$
(362) $7(R)$

(363) $7(S)$<smiles>C/C(=C\CCC(C)CO)c1ccc(C(=O)O)cc1O</smiles>

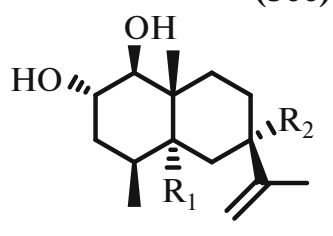

Penicieudesmol A-D

(369) $R_{1}=H, R_{2}=H$

(370) $\mathrm{R}_{1}=\mathrm{H}, \mathrm{R}_{2}=\mathrm{OH}$

(371) $\mathrm{R}_{1}=\mathrm{OH}, \mathrm{R}_{2}=\mathrm{H}$

(372) $\mathrm{R}_{1}=\mathrm{OH}, \mathrm{R}_{2}=\mathrm{OH}$
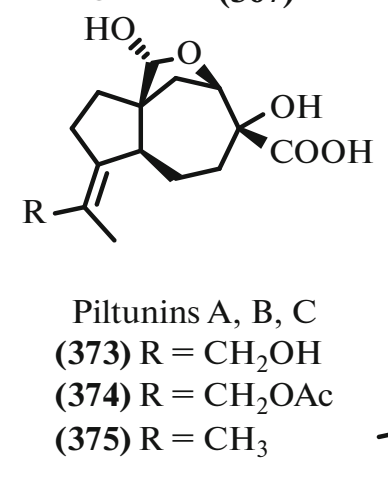

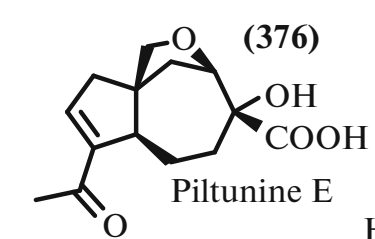

(364)<smiles>CC(C)C(O)CCC(O)(O)c1ccc(C(=O)O)cc1O</smiles>

(365)

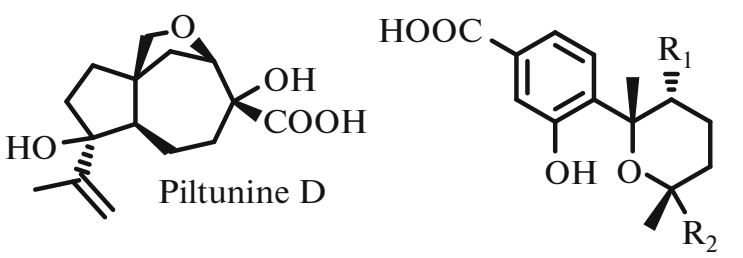

(379) $\mathrm{R}_{1}=\mathrm{OH}, \mathrm{R}_{2}=\mathrm{CH}_{3}$

(380) $\mathrm{R}_{1}=\mathrm{H}, \mathrm{R}_{2}=\mathrm{CH}_{3}$

(381) $\mathrm{R}_{1}=\mathrm{H}, \mathrm{R}_{2}=\mathrm{CH}_{2} \mathrm{OH}$

(382) $\mathrm{R}_{1}=\mathrm{H}, \mathrm{R}_{2}=\mathrm{CH}_{2} \mathrm{OAc}$

(377)<smiles>C=C(CCCC(C)(C)O)c1ccc(C(=O)O)cc1O</smiles>

(383)<smiles>C=C(CCC(C)(C)C)CC(C)O</smiles>

(378)<smiles>CC1(C)[C@@H](O)[C@@H](O)C[C@]2(C)CC(C(=O)O)=CC[C@@]12C</smiles>

(387)
Cyclonerodiole A

Cyclonerod
$(390)$

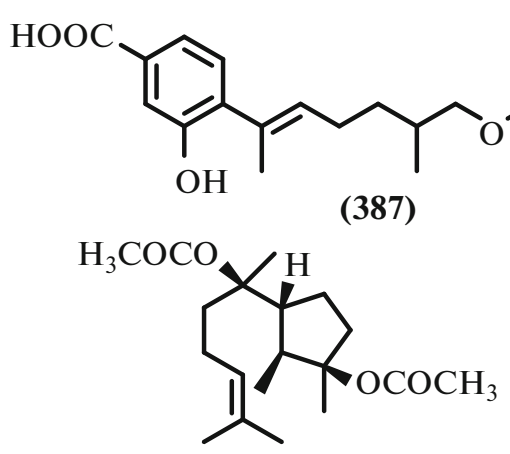<smiles>CC(C)=O</smiles><smiles>CC=C(C)C(=O)O</smiles><smiles>Cc1cccc(O)c1</smiles><smiles>CO[C@@]1(C)CC[C@H](C(C)C)[C@H]2[C@@H]1CC[C@@]2(C)OC</smiles>

Cyclonerodiole B (391)<smiles>C=CC(=C)[C@@H](O)CCCC(C)(C)O</smiles>

(388)<smiles>CC1=CCC(C(C)CCC(O)C(C)(C)O)C=C1</smiles>

Trichodermaerin A

(392)
9,10-Diolhinokiic acid

(389)

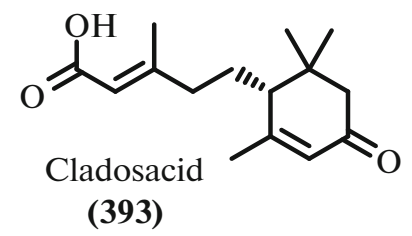

Fig. 25. Sesquiterpenes and sesquiterpenoids. 


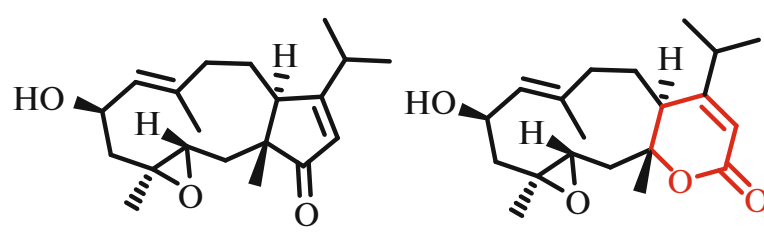

Stachatranone A (394)<smiles>CC(=O)CC(/C=C(\C)[C@H](O)C/C=C1/C(C(C)C)=CC(=O)O[C@]1(C)CC[C@@H](C)C(=O)C(C)C)C(C)C</smiles>

Atranone Q

(397)

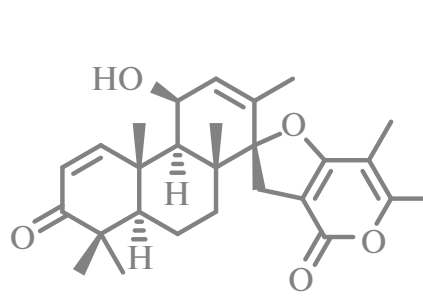

Brevione I (402)
Stachatranone B (395)

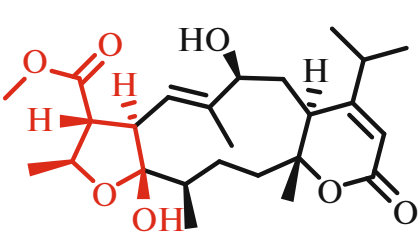

Atranone R

(398)

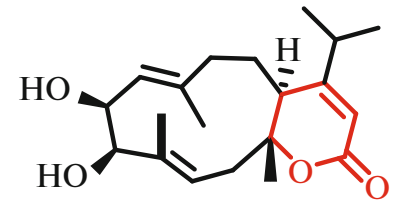

Stachatranone C (396)

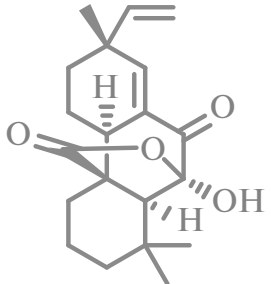

Aspergiloid E (400)

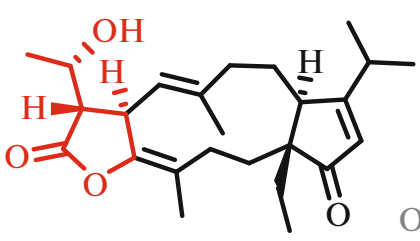

Atranone $\mathrm{S}$

(399)

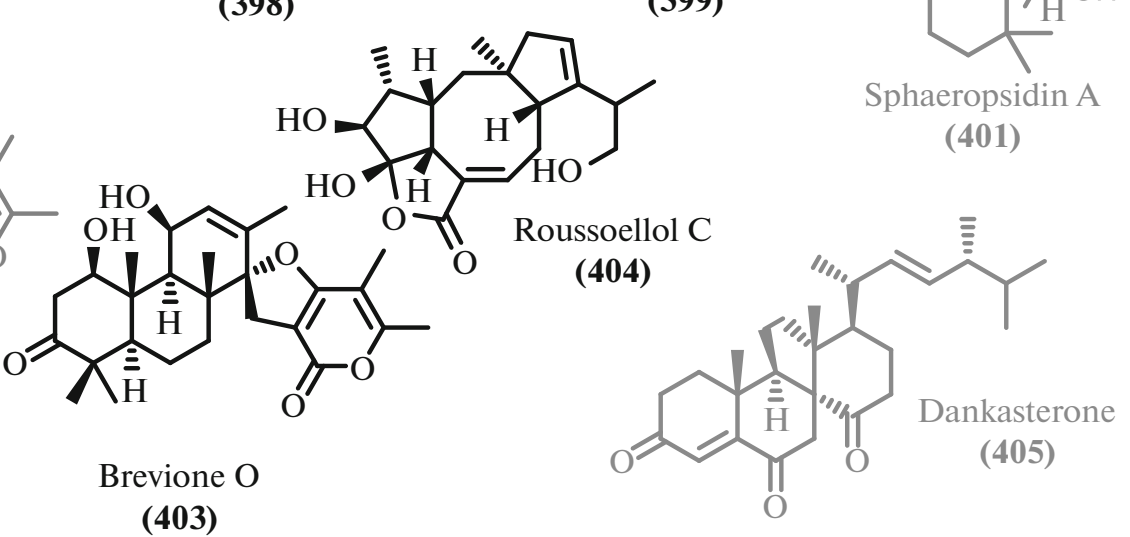

(403)

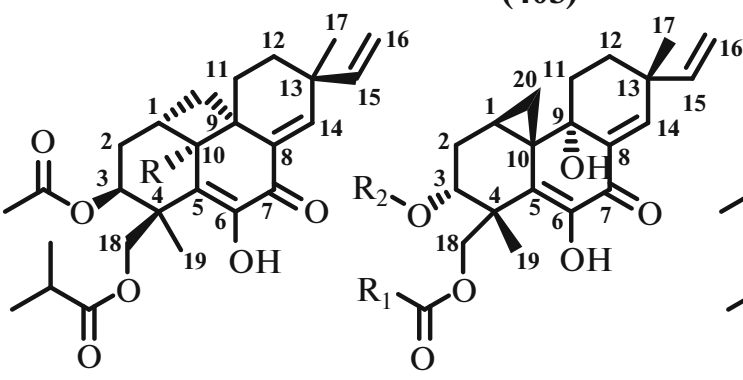

Eutypellenone A (406) $\mathrm{R}=\mathrm{OH}$

Eutypellenone $\mathrm{B}$ (407) $\mathrm{R}=\mathrm{OCH}_{3}$

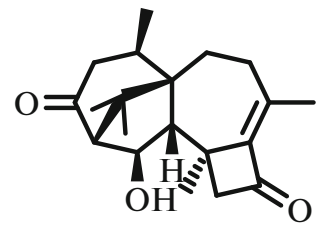

Harziandione A

(420)

Libertellenone $\mathrm{P}$

Libertellenone $\mathrm{Q}$

Libertellenone $\mathrm{R}$

(413) $\mathrm{R}=\mathrm{i}-\mathrm{C}_{3} \mathrm{H}_{7}, \mathrm{R}_{2}=\mathrm{H}$

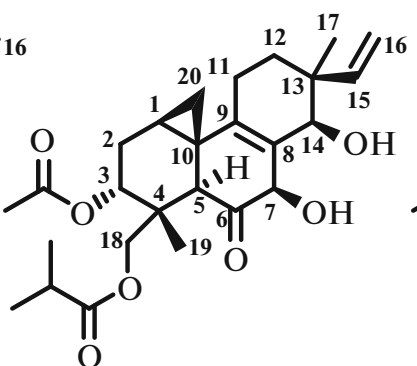

Libertellenon S

(414)

(408) $\mathrm{R}=\mathrm{i}-\mathrm{C}_{3} \mathrm{H}_{7}, \mathrm{R}_{2}=\mathrm{COCH}_{3}$

(411) $\mathrm{R}=\mathrm{CH}_{3}, \mathrm{R}_{2}=\mathrm{COCH}_{3}$

(412) $\mathrm{R}=\mathrm{C}_{2} \mathrm{H}_{5}, \mathrm{R}_{2}=\mathrm{COCH}_{3}$

Libertellenon J

(409)
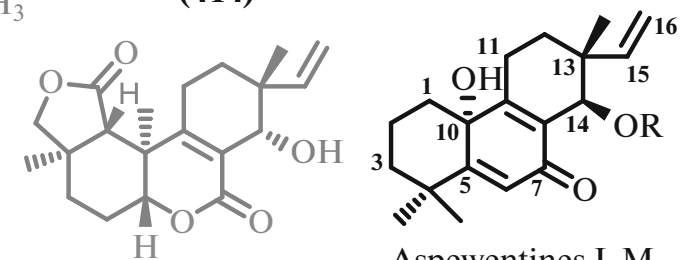

Aspewentines I-M

(415) $10(R), 14(R) \mathrm{R}=\mathrm{H}$

(416) $10(R), 14(S) \mathrm{R}=\mathrm{H}$

(417) $10(S), 14(S) \mathrm{R}=\mathrm{H}$

(418) $10(S), 14(R) \mathrm{R}=\mathrm{H}$

(419) $10(S), 14(S) \mathrm{R}=\mathrm{CH}_{3}$

Fig. 26. Diterpenes and diterpenoids. 


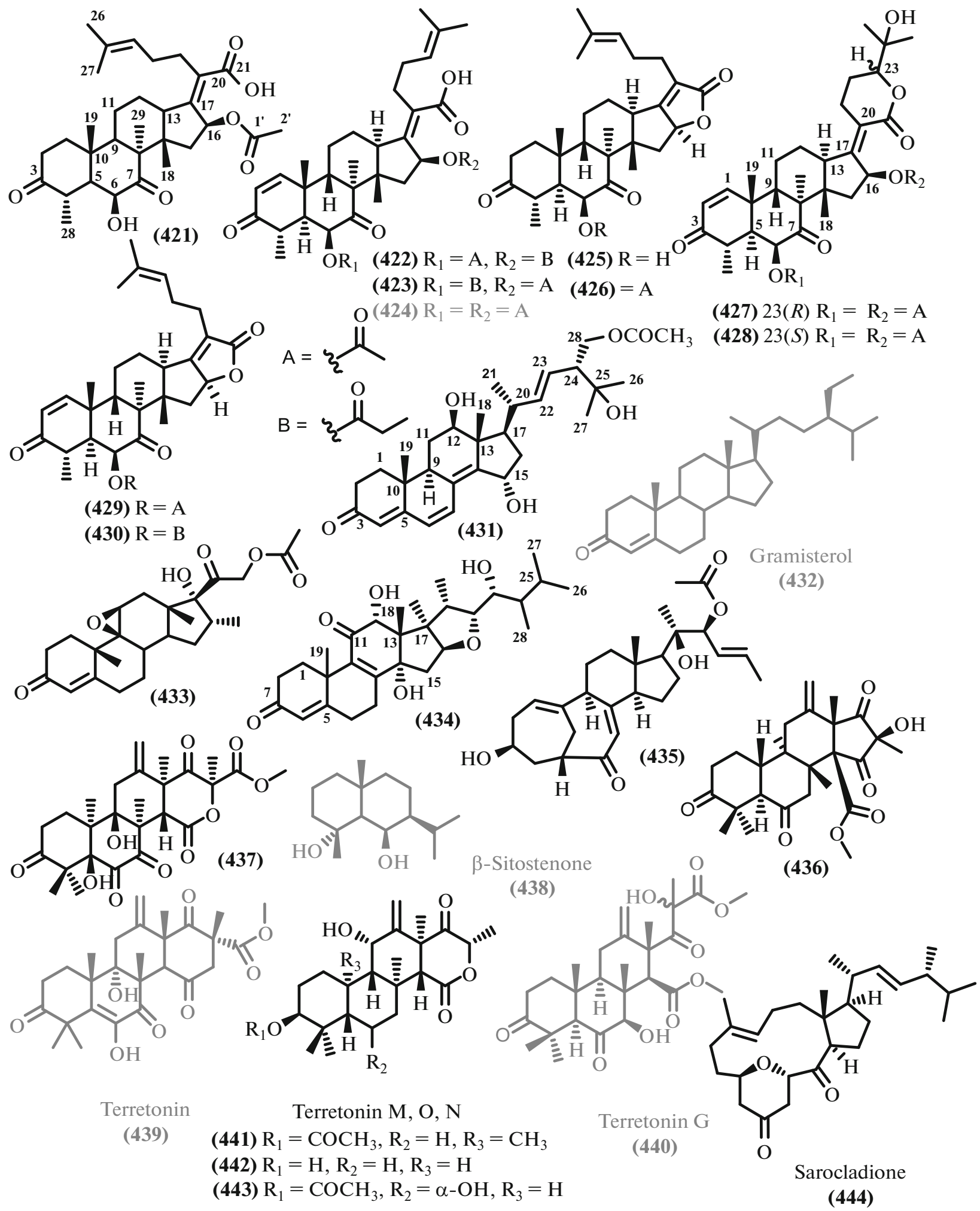

Fig. 27. Steroids and their derivatives.

\section{COMPOUNDS OF MIXED AND UNIDENTIFIED ORIGIN}

In addition to the compounds summarized in the previous chapters, antibiotic secondary metabolites have been identified, which are difficult to classify unambiguously into one of the abovementioned classes of compounds. In some cases, it is obvious that 


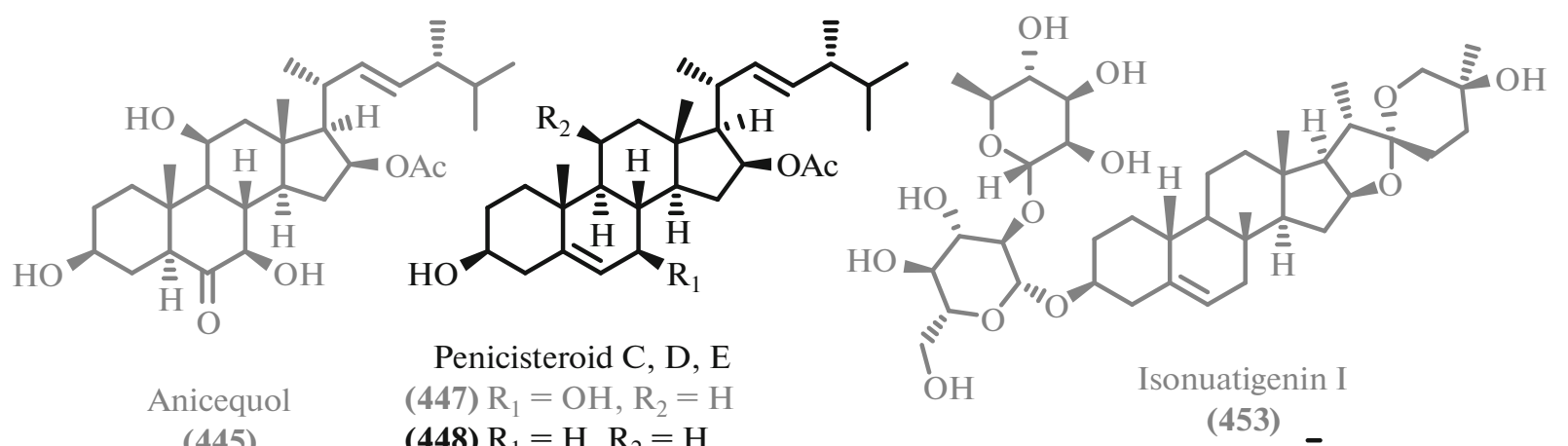
(445)

(448) $\mathrm{R}_{1}=\mathrm{H}, \mathrm{R}_{2}=\mathrm{H}$ (453)

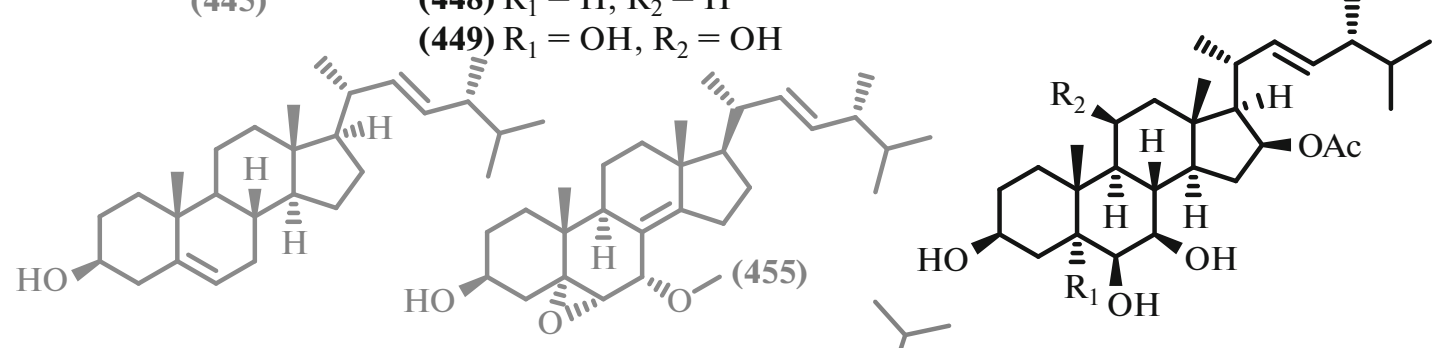<smiles>C=C(CCC(C)C1CCC2C3=CCC4C(C)[C@@H](O)CC[C@]4(C)C3CC[C@@]21C)C(C)C</smiles>

Penicisteroid A, F, G, H (446) $\mathrm{R}_{1}=\mathrm{H}, \mathrm{R}_{2}=\mathrm{OH}$ (450) $\mathrm{R}_{1}=\mathrm{OH}, \mathrm{R}_{2}=\mathrm{OH}$ (451) $\mathrm{R}_{1}=\mathrm{OH}, \mathrm{R}_{2}=\mathrm{H}$ (452) $\mathrm{R}_{1}=\mathrm{H}, \mathrm{R}_{2}=\mathrm{H}$

Fig. 28. Sterols and their derivatives.

the biosynthesis of these compounds requires the joint work of enzyme machinery of different classes (polyketide synthases, nonribosomal peptide synthases, fatty acid synthases, sterol biosynthesis enzymes, the shikimate pathway, etc.). Sometimes (especially for structurally simple compounds), there are not enough data for the biogenetic attribution.

When studying the antimicrobial activity of the extract culture of marine micromycete Penicillium ZZ1283 [301], eighteen metabolites were isolated and their structures were determined. One of them turned out to be previously undescribed compound, purpuride $\mathrm{D}(\mathbf{4 5 8})$, which was a conjugate of drimane-type sesquiterpene and amino acid $N$-acetyl-L-valine.

Two known micotoxins, ochratoxins A (464) and B (465) $[302,303]$, that contained the peptide and polyketide fragments were isolated from marine fungus Aspergillus ochraceus [42]. Ochratoxins A ethyl ester (461) and its antibiotic activity were studied when isolated from mangrove fungus Aspergillus sp. SCSIO41211 [254].
Producer Trichoderma erinaceum F1-1 [270] of marine origin mentioned in Section 4 significantly changed the spectrum of synthesized secondary metabolites when cultured on a medium in the presence of L-tryptophan. Instead of terpenoids, eighteen aromatic compounds were isolated in this case, including six new compounds, trichoderolides $\mathrm{A}-\mathrm{F}$ (462-467), of which only compound (463) showed weak cytotoxicity. The known cytotoxic aromatic compounds, i.e., 3,3'-dimethoxy-5,5'-dimethyldiphenyl ether (468) [304], 3-O-methyldiorcinol (469) [305], and isolated from a natural source for the first time 4'-[(2-hydroxy-1,3-propanyl)bis[oxy-4,1-phenylene(1-methylethylidene)]]bisphenol (470) [306] were also obtained.

Chemical study of the extract of marine fungus Truncatella angustata [307] led to the isolation of previously undescribed prenylated cyclohexanols, two of which $(\mathbf{4 7 1}, \mathbf{4 7 2})$ showed antiviral activity.

Penicillilactone A (473) was isolated from marine fungus Penicillium sp. LS54 [308], which was the first natural compound that contained the 7-membered lactone cycle condensed with the furan ring. 
Table 4. Data on the origin and biological activity of polypeptides of various structural types

\begin{tabular}{|c|c|c|c|c|c|}
\hline Cpd. & Producer/species & $\begin{array}{l}\text { Extremophile } \\
\text { type/origin }\end{array}$ & $\begin{array}{c}\text { Activity/ } \\
\text { toxicity }\end{array}$ & $\mathrm{EC}_{50}, \mathrm{IC}_{50}, \mathrm{MIC}$ & Ref. \\
\hline 257 & Curvularia sp. & Marine & $\begin{array}{l}\mathrm{CT} \\
\mathrm{AB}\end{array}$ & $\begin{array}{l}\text { IC }_{50} 12.46 \mu \mathrm{M}(\mathrm{BEL} 7402 / 5-\mathrm{Fu}) \\
\text { MIC 62.5 } \boldsymbol{\mu M} \text { (P. gingivalis })\end{array}$ & [219] \\
\hline 161 & Zopfiella marina & Marine & $\begin{array}{l}\mathrm{AT} \\
\mathrm{AB} \\
\mathrm{CT}\end{array}$ & $\begin{array}{l}\text { MIC } 25 \mu \mathrm{g} / \mathrm{mL} \text { (M. tuberculosis H37Ra) } \\
\text { MIC } 12.5 \mu \mathrm{g} / \mathrm{mL} \text { (B. cereus) } \\
\text { IC }_{50} 13.6 \mu \mathrm{g} / \mathrm{mL} \text { (Vero) }\end{array}$ & [164] \\
\hline 199 & $\begin{array}{l}\text { Aspergillus fumigatus } \\
\text { MF029 }\end{array}$ & Marine & $\begin{array}{l}\mathrm{AB} \\
\mathrm{AT} \\
\mathrm{AF}\end{array}$ & $\begin{array}{l}\text { MIC 50, 50, 12.5, } 1.25 \mu \mathrm{g} / \mathrm{mL} \text { (MRSA, S. aureus, } \\
\text { B. subtilis) } \\
\text { MIC } 1.25 \mu \mathrm{g} / \mathbf{m L}(\mathrm{BCG}) \\
\text { MIC 8, 32, 8, } 16 \boldsymbol{\mu g} / \mathbf{m L} \text { (Cryptococcus neoformans MY } \\
\text { 1051, C.neoformans MY 1146, C. neoformans MY 2061, } \\
\text { C. neoformans MY 2062) [207] } \\
\text { MIC 128 } \boldsymbol{\mu g} / \mathrm{mL} \text { (C. albicans MY 1028, MY 1055, } \\
\text { MY 1750) [207] } \\
\text { MIC 128, 16, 128, } 128 \mu \mathrm{g} / \mathbf{m L} \text { (C. parapsilosis MY 1010, } \\
\text { C. pseudotropicalis MY 2099, C. tropicalis MY 1012, } \\
\text { S. cerevisiae MY 1976) [207] } \\
\text { MIC } 64 \boldsymbol{\mu g} / \mathrm{mL} \text { (A. flavus MF 383) }\end{array}$ & [75] \\
\hline 201 & Aspergillus sp. & Marine & $\begin{array}{l}\mathrm{AB} \\
\mathrm{CT}\end{array}$ & $\begin{array}{l}\text { MIC } 15.15,30.30,7.53 \mu \mathrm{M}(\text { V. anguillarum, } \\
\text { A. salmonicida, P. aeruginosa) } \\
\text { IC }_{50} 3.56-\mathbf{1 0 . 6 9} \boldsymbol{\mu M} \text { (Jurkat, A549, HeLa) }\end{array}$ & [74] \\
\hline 248 & $\begin{array}{l}\text { Aspergillus terreus } \\
\text { S020 }\end{array}$ & Marine & $\mathrm{CT}$ & 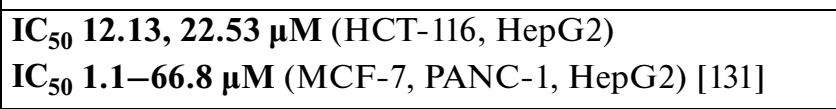 & [226] \\
\hline \begin{tabular}{l|}
111 \\
112 \\
155
\end{tabular} & $\begin{array}{l}\text { Alternaria sp. } \\
\text { SCSIO41014 }\end{array}$ & Marine & $\begin{array}{l}\mathrm{CT} \\
\mathrm{AB}\end{array}$ & 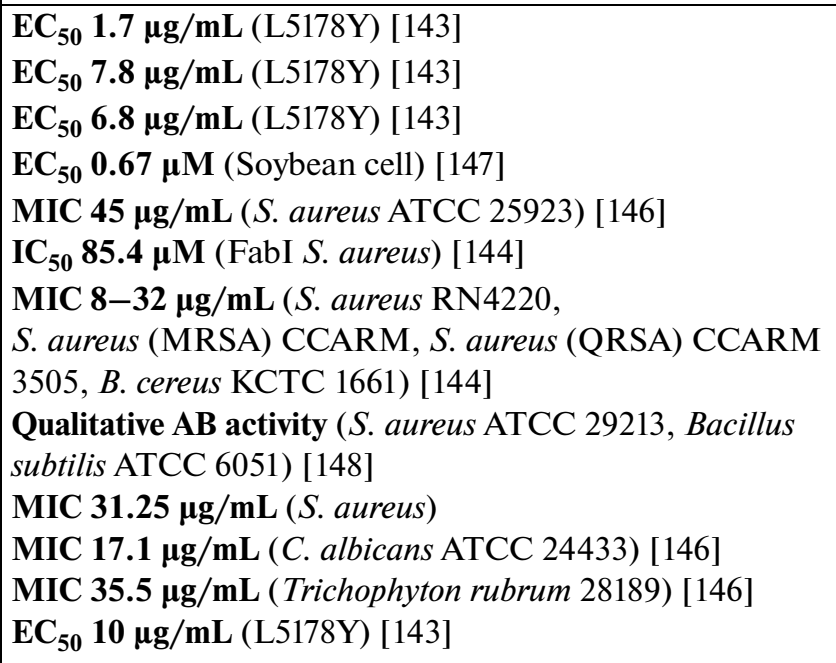 & [87] \\
\hline 196 & $\begin{array}{l}\text { Penicillium sp. } \\
\text { ZZ901 }\end{array}$ & Marine & $\begin{array}{l}\mathrm{CT} \\
\mathrm{AB}\end{array}$ & $\begin{array}{l}\text { IC }_{50} 60.93 \mu \mathrm{M} \text { (U87MG glioma cells) } \\
\mathrm{IC}_{50} 60.81 \mu \mathrm{M} \text { (C6 glioma cells) } \\
\mathrm{MIC}_{23,35 \mu \mathrm{g} / \mathrm{mL} \text { (MRSA, } \text { E. coli) }} \\
\mathrm{IC}_{50} 55.99 \mu \mathrm{M} \text { (U87MG glioma cells) } \\
\mathrm{IC}_{50} 44.65 \mu \mathrm{M} \text { (C6 glioma cells) } \\
\text { IC }_{50} 37.26 \mu \mathrm{M} \text { (U87MG glioma cells) } \\
\text { IC }_{50} 23.24 \mu \mathrm{M} \text { (C6 glioma cells) } \\
\mathrm{MIC} 7,9 \mu \mathrm{g} / \mathrm{mL} \text { (MRSA, E. coli) }\end{array}$ & [94] \\
\hline 207 & $\begin{array}{l}\text { Penicillium sclerotio- } \\
\text { rum GDST-2013- } \\
0415 \\
\end{array}$ & Marine & AV & $\mathrm{IC}_{50}$ 19.5-132 $\boldsymbol{\mu M}(\mathrm{HSV}, \mathrm{EV} 71, \mathrm{RSV} 1)$ & [198] \\
\hline
\end{tabular}


Table 4. (Contd.)

\begin{tabular}{|c|c|c|c|c|c|}
\hline Cpd. & Producer/species & $\begin{array}{l}\text { Extremophile } \\
\text { type/origin }\end{array}$ & $\begin{array}{l}\text { Activity/ } \\
\text { toxicity }\end{array}$ & $\mathrm{EC}_{50}, \mathrm{IC}_{50}, \mathrm{MIC}$ & Ref. \\
\hline 210 & \multirow[t]{4}{*}{ Penicillium canescens } & \multirow[t]{4}{*}{ Marine } & \multirow[t]{4}{*}{$\begin{array}{l}\mathrm{CT} \\
\mathrm{AF} \\
\mathrm{AB}\end{array}$} & $\mathrm{IC}_{50} 13.9 \boldsymbol{\mu M}(\mathrm{L} 5178 \mathrm{Y})$ & \\
\hline 211 & & & & $\mathrm{IC}_{\mathbf{5 0}} \mathbf{2 . 7}, \mathbf{8 . 9} \boldsymbol{\mu M}(\mathrm{A} 2780, \mathrm{~L} 5178 \mathrm{Y})$ & \\
\hline 200 & & & & $\begin{array}{l}\text { IC }_{50} \mathbf{2 0 , 3 5 \mu M}(\text { HeLa, SCC-114) }[131] \\
\text { IC }_{50} 11.6 \mu \mathrm{g} / \mathbf{m L}(\text { C. gloeosporioides })[195] \\
\text { IC }_{50} 2.68 \mu \mathrm{g} / \mathbf{m L}(\text { A. solani })[195]\end{array}$ & \\
\hline 249 & & & & $\begin{array}{l}\text { IC }_{\mathbf{5 0}} \mathbf{8 . 9} \boldsymbol{\mu M} \text { (L5178Y) } \\
\text { IC }_{\mathbf{5 0}} \mathbf{7 . 8 - 2 9 . 4} \boldsymbol{\mu M}(\mathrm{POS} 1, \text { AT6-1, L929) [217] } \\
\text { MIC } 111.1 \mu \mathrm{g} / \mathbf{m L} \text { (Agrobacterium tumefaciens, } \\
\text { Clavibacter michiganensis subsp. michiganensis, } \\
\text { Pectobacterium carotovorum subsp. carotovorum, } \\
\text { Pseudomonas syringae pv. Actinidae, Xanthomonas } \\
\text { arboricola pv. Pruni, X. axonopodis pv. citri) [218] } \\
\text { MIC } 37 \boldsymbol{\mu g} / \mathbf{m L} \text { (Acidovorax avenae subsp. cattlyae, } \\
\text { Burkholderia glumae, X. euversicatoria, Streptomyces } \\
\text { scabies) }[218] \\
\text { MIC } 12.3 \mu \mathrm{g} / \mathrm{mL}(\text { X. oryzae pv. oryzae) [218] }\end{array}$ & {$[176]$} \\
\hline 212 & \multirow{8}{*}{$\begin{array}{l}\text { Diaporthe sp. SCSIO } \\
41011\end{array}$} & \multirow[t]{8}{*}{ Mangrove } & \multirow{8}{*}{$\begin{array}{l}\text { CT } \\
\text { AP }\end{array}$} & $\mathrm{IC}_{50} \mathbf{2 7 - 4 5} \boldsymbol{\mu M}(\mathrm{ACHN}, 786-\mathrm{O},-\mathrm{RC}-2)$ & \multirow{8}{*}[200]{} \\
\hline 214 & & & & $\mathrm{IC}_{\mathbf{5 0}} \mathbf{3 8}, \mathbf{4 4} \boldsymbol{\mu M}(786-\mathrm{O}, \mathrm{OS}-\mathrm{RC}-2)$ & \\
\hline 215 & & & & $\mathrm{IC}_{\mathbf{5 0}}$ 8.9-14 $\boldsymbol{\mu M}(\mathrm{ACHN}, 786-\mathrm{O}, \mathrm{OS}-\mathrm{RC}-2)$ & \\
\hline 204 & & & & IC $_{50} \mathbf{1 0 - 3 8} \boldsymbol{\mu M}(\mathrm{ACHN}, 786-\mathrm{O}, \mathrm{OS}-\mathrm{RC}-2)$ & \\
\hline 216 & & & & $\mathrm{IC}_{50} 48,4.9 \mu \mathrm{M}(786-\mathrm{O}, \mathrm{OS}-\mathrm{RC}-2)$ & \\
\hline & & & & $\begin{array}{l}\text { IC }_{50} \text { 6.2-10.6 } \boldsymbol{~} \mathbf{g} / \mathbf{m L}(\mathrm{KB}, \mathrm{NCI}-\mathrm{H} 187, \mathrm{MCF}-7)[201] \\
\mathrm{IC}_{\mathbf{5 0}} 7.8 \boldsymbol{\mu g} / \mathbf{m L}(\text { Plasmodium falciparum })[201]\end{array}$ & \\
\hline 217 & & & & 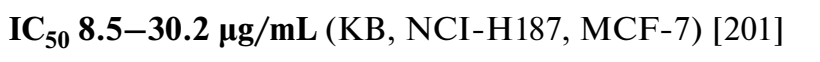 & \\
\hline 205 & & & & $\mathrm{IC}_{\mathbf{5 0}}$ 3.0-4.4 $\boldsymbol{\mu M}(\mathrm{ACHN}, 786-\mathrm{O}, \mathrm{OS}-\mathrm{RC}-2)$ & \\
\hline 145 & $\begin{array}{l}\text { Penicillium purpuro- } \\
\text { genum G59 }\end{array}$ & Marine & CT & $\mathrm{IC}_{\mathbf{5 0}} \mathbf{1 2 4 . 3 , 3 2 9 . 9 \mu \mathrm { M } ( \mathrm { HL } - 6 0 , \mathrm { K } 5 6 2 )}$ & {$[159]$} \\
\hline 177 & \multirow{3}{*}{$\begin{array}{l}\text { Pseudopestalotiopsis } \\
\text { sp. PSU-AMF45 }\end{array}$} & \multirow[t]{3}{*}{ Marine } & \multirow[t]{3}{*}{$\mathrm{AF}$} & MIC $200 \mu \mathrm{g} / \mathrm{mL}$ (Cryptococcus neoformans ATCC90112) & \multirow{3}{*}[102]{} \\
\hline 178 & & & & MIC $200 \mu \mathrm{g} / \mathrm{mL}$ (Cryptococcus neoformans ATCC90112) & \\
\hline 136 & & & & $\mathbf{I C}_{\mathbf{5 0}} \mathbf{0 . 0 1} \boldsymbol{\mu M}(\mathrm{HEP}-\mathrm{G} 2)[154]$ & \\
\hline 245 & $\begin{array}{l}\text { Penicillium janthinel- } \\
\text { lum }\end{array}$ & Marine & $\mathrm{CT}$ & $\begin{array}{l}\text { GI }_{\mathbf{5 0}} \text { 9.3-31 } \boldsymbol{\mu M}(\mathrm{NUGC}-3, \mathrm{HCT}-15, \mathrm{NCI}-\mathrm{H} 23, \\
\mathrm{ACHN}, \mathrm{PC}-3, \mathrm{MDA}-\mathrm{MB}-231)\end{array}$ & {$[208]$} \\
\hline 139 & $\begin{array}{l}\text { Curvularia sp. IFB- } \\
\text { Z10 }\end{array}$ & Marine & CT & 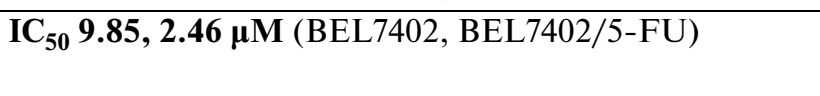 & {$[155]$} \\
\hline 124 & Penicillium piltun- & Marine & CT & $\mathrm{IC}_{50} \mathbf{7 1 . 7 4} \boldsymbol{\mu M}(22 \mathrm{Rv} 1)$ & {$[151]$} \\
\hline 125 & ense КMМ 4668 & & AF & $\mathbf{I C}_{50} \mathbf{3 0} \boldsymbol{\mu M}(P$. viticola $)$ & \\
\hline 252 & $\begin{array}{l}\text { Cladosporium sp. } \\
\text { OUCMDZ-1635 }\end{array}$ & Marine & $\mathrm{CT}$ & IC $_{50}$ 9.1-19.1 $\boldsymbol{\mu M}$ (MCF-7, HeLa, HCT-116, HL-60) & [220] \\
\hline 255 & $\begin{array}{l}\text { Aspergillus protu- } \\
\text { berus MUT } 3638\end{array}$ & Marine & $\mathrm{AB}$ & MIC $30 \mu \mathrm{g} / \mathrm{mL}$ (S. aureus) & {$[222]$} \\
\hline 148 & $\begin{array}{l}\text { Colletotrichum } \\
\text { gloeosporioides }\end{array}$ & Mangrove & $\mathrm{AB}$ & $\begin{array}{l}\text { MIC } 25,12.5,12.5,12.5 \mu \mathrm{g} / \mathrm{mL} \text { (B. cereus, B. subtilis, } \\
\text { S. aureus, } S . \text { albus) } \\
\text { MIC } 25 \mu \mathrm{g} / \mathrm{mL} \text { (B. cereus, B. subtilis, S. aureus) }\end{array}$ & {$[113]$} \\
\hline
\end{tabular}


Table 4. (Contd.)

\begin{tabular}{|c|c|c|c|c|c|}
\hline Cpd. & Producer/species & $\begin{array}{l}\text { Extremophile } \\
\text { type/origin }\end{array}$ & $\begin{array}{l}\text { Activity/ } \\
\text { toxicity }\end{array}$ & $\mathrm{EC}_{50}, \mathrm{IC}_{50}, \mathrm{MIC}$ & Ref. \\
\hline $\begin{array}{c}154 \\
151 \\
149 \\
153 \\
160\end{array}$ & $\begin{array}{l}\text { Dothiorella sp. } \\
\text { ML002 }\end{array}$ & Mangrove & $\begin{array}{l}\text { AB } \\
\text { CT } \\
\text { AV }\end{array}$ & 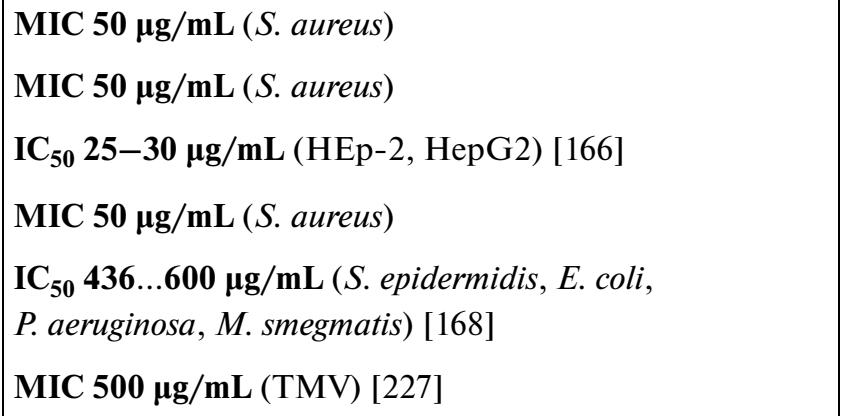 & [165] \\
\hline 121 & Pestalotiopsis sp. & Mangrove & $\mathrm{AB}$ & MIC 12.5, $50 \mu \mathrm{g} / \mathrm{mL}($ E. coli,$P$. aeruginosa $)$ & [145] \\
\hline $\begin{array}{l}119 \\
120\end{array}$ & $\begin{array}{l}\text { Ascomycota sp. } \\
\text { CYSK-4 }\end{array}$ & Mangrove & $\begin{array}{l}\mathrm{AB} \\
\mathrm{AF} \\
\mathrm{CT}\end{array}$ & 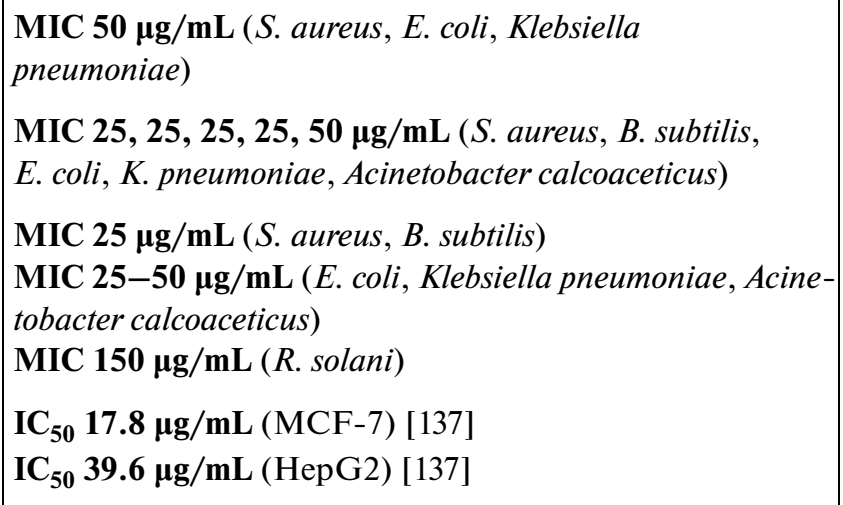 & [136] \\
\hline $\begin{array}{r}140 \\
142\end{array}$ & $\begin{array}{l}\text { Lasiodiplodia theo- } \\
\text { bromae M4.2-2 }\end{array}$ & Mangrove & $\begin{array}{l}\mathrm{CT} \\
\mathrm{AB}\end{array}$ & 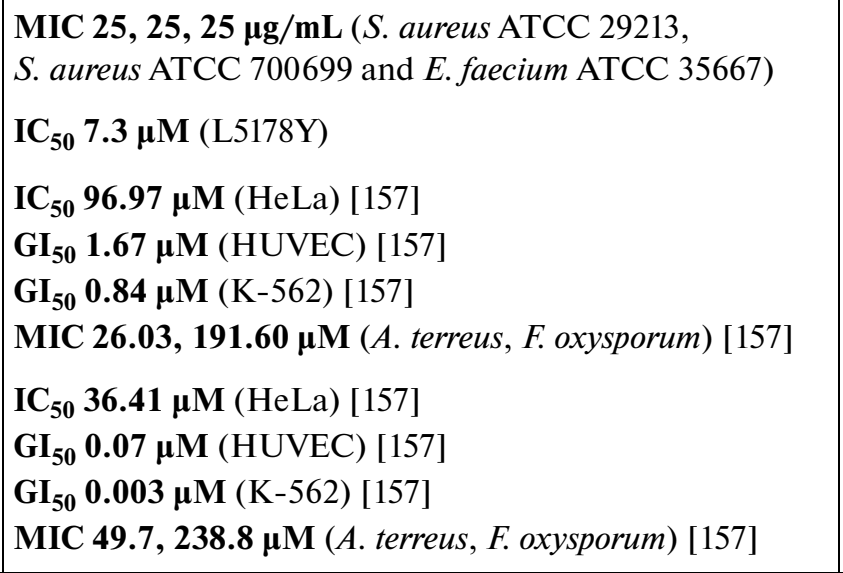 & [156] \\
\hline $\begin{array}{l}118 \\
158\end{array}$ & $\begin{array}{l}\text { Phyllosticta capitalen- } \\
\text { sis }\end{array}$ & Mangrove & $\mathrm{AB}$ & $\begin{array}{l}\text { MIC } 25 \mu \mathrm{g} / \mathrm{mL} \text { ( } \text {. a aeruginosa) } \\
\text { MIC } 25 \mu \mathrm{g} / \mathrm{mL} \text { ( } \text { P. aeruginosa) }\end{array}$ & [141] \\
\hline $\begin{array}{l}247 \\
170 \\
169\end{array}$ & $\begin{array}{l}\text { Cladosporium } \mathrm{sp} . \\
\mathrm{JS} 1-2\end{array}$ & Mangrove & $\begin{array}{l}\mathrm{AB} \\
\mathrm{CT}\end{array}$ & 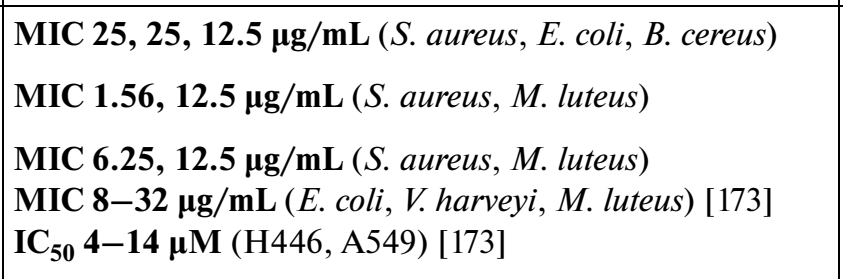 & {$[121]$} \\
\hline 218 & $\begin{array}{l}\text { Penicillium sp. } \\
\text { ZZ380 } \\
\text { Cladosporium sp. } \\
\text { JS1-2 }\end{array}$ & $\begin{array}{l}\text { Marine } \\
\text { Mangrove }\end{array}$ & $\mathrm{AB}$ & $\begin{array}{l}\text { MIC 6.25, 12.5, } 12.5 \mu \mathrm{g} / \mathrm{mL}(S . \text { aureus, E. coli, } \\
\text { B. cereus) }\end{array}$ & $\begin{array}{r}{[78]} \\
{[121]}\end{array}$ \\
\hline
\end{tabular}


Table 4. (Contd.)

\begin{tabular}{|c|c|c|c|c|c|}
\hline Cpd. & Producer/species & $\begin{array}{l}\text { Extremophile } \\
\text { type/origin }\end{array}$ & $\begin{array}{l}\text { Activity/ } \\
\text { toxicity }\end{array}$ & $\mathrm{EC}_{50}, \mathrm{IC}_{50}, \mathrm{MIC}$ & Ref. \\
\hline 138 & $\begin{array}{l}\text { Penicillium pinophi- } \\
\text { lum } \\
\text { SCAU037 }\end{array}$ & Mangrove & $\begin{array}{l}\mathrm{AB} \\
\mathrm{AF} \\
\mathrm{CT}\end{array}$ & 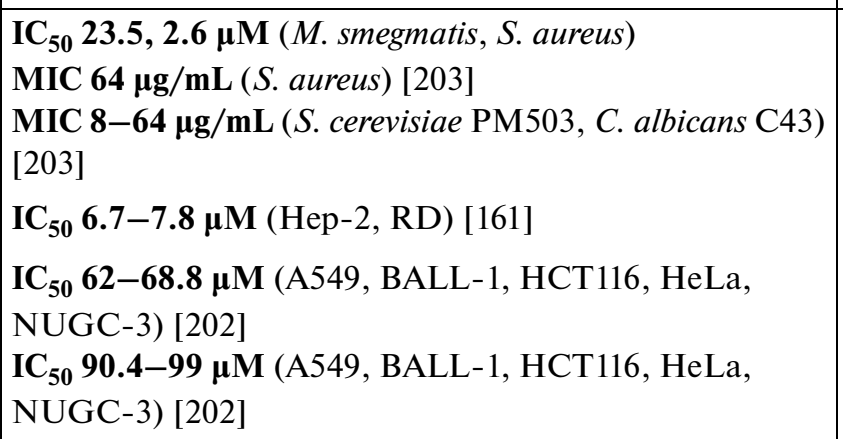 & [130] \\
\hline 157 & $\begin{array}{l}\text { Aspergillus sp. } \\
\mathrm{AV}-2\end{array}$ & Mangrove & CT & $\mathrm{IC}_{50} 2.87 \mu \mathrm{M}(\mathrm{Caco}-2)$ & {$[170]$} \\
\hline 241 & Fusarium solani $\mathrm{H} 918$ & Mangrove & $\mathrm{AF}$ & $\mathbf{E D}_{50} 55 \mu \mathrm{M}(P$. theae $)$ & [211] \\
\hline $\begin{array}{l}129 \\
130\end{array}$ & $\begin{array}{l}\text { Cladosporium } \\
\text { cladosporioides MA- } \\
299\end{array}$ & Mangrove & $\begin{array}{l}\mathrm{AB} \\
\mathrm{AF}\end{array}$ & $\begin{array}{l}\text { MIC } 2 \mu \mathrm{g} / \mathrm{mL} \text { (C. glecosporioides) } \\
\text { MIC } 1,8 \mu \mathrm{g} / \mathrm{mL} \text { (Edwardsiella tarda, E. ictarda) } \\
\text { MIC } 2,8 \mu \mathrm{g} / \mathrm{mL} \text { (C. glecosporioides, Bipolaris sorokiniana) } \\
\text { MIC } 2 \mu \mathrm{g} / \mathrm{mL} \text { (C. glecosporioides) } \\
\text { MIC } 32 \mu \mathrm{g} / \mathrm{mL} \text { (P. piricola Nose) } \\
\text { MIC } 1 \mu \mathrm{g} / \mathrm{mL} \text { (Fusarium oxysporum f. sp. cucumerinum) } \\
\text { MIC } 1 \mu \mathrm{g} / \mathrm{mL} \text { (C. glecosporioides) } \\
\text { MIC } 32 \mu \mathrm{g} / \mathrm{mL} \text { (P. piricola Nose) } \\
\text { MIC } 32 \mu \mathrm{g} / \mathrm{mL} \text { (Fusarium oxysporum f. sp. cucumerinum) } \\
\text { MIC } 1 \mu \mathrm{g} / \mathrm{mL} \text { (E. ictarda) } \\
\text { MIC } 1 \mu \mathrm{g} / \mathrm{mL} \text { (C. glecosporioides) } \\
\text { MIC } 32 \mu \mathrm{g} / \mathbf{m L} \text { (P. piricola Nose) } \\
\text { MIC } 32 \mu \mathrm{g} / \mathbf{m L} \text { (Fusarium oxysporum f. sp. cucumerinum) }\end{array}$ & [152] \\
\hline $\begin{array}{l}224 \\
225 \\
227 \\
228\end{array}$ & $\begin{array}{l}\text { Cocultivation } \\
\text { Penicillium crustosum } \\
\text { PRB-2 and Xylaria } \\
\text { sp. HDN13-249 }\end{array}$ & $\begin{array}{l}\text { Antarctica } \\
\text { Mangrove }\end{array}$ & $\mathrm{AB}$ & $\begin{array}{l}\text { MIC } 100 \mu \mathrm{M} \text { (B. subtilis) } \\
\text { MIC 6.25, 12.5 } \mu \mathrm{M}(\text { M. phlei, V. parahemolyticus }) \\
\text { MIC } 25 \mu \mathrm{M}(\text { M. phlei) } \\
\text { MIC 12.5, } 25 \mu \mathrm{M} \text { (M. phlei, V. parahemolyticus })\end{array}$ & [213] \\
\hline 162 & $\begin{array}{l}\text { Penicillium sp. } \\
\text { RO-11 }\end{array}$ & $\begin{array}{l}\text { Hot spring } \\
\text { deposits } \\
\left(45-65^{\circ} \mathrm{C}\right), \\
\text { Saudi Arabia/ } \\
\text { Thermophile }\end{array}$ & $\begin{array}{l}\mathrm{AB} \\
\mathrm{CT}\end{array}$ & $\begin{array}{l}\text { MIC 9.3, } 7.4 \mu \mathrm{g} / \mathrm{mL} \text { (Enterobacter xiangfangensis, } \\
\text { P. aeruginosa) } \\
\text { MIC } 6.3,5.7 \mu \mathrm{g} / \mathrm{mL} \text { (Escherichia fergusonii, } \\
\begin{array}{l}\text { P. aeruginosa) } \\
\text { IC }_{50} 22 \boldsymbol{M} \text { М (HTB-176) }\end{array}\end{array}$ & [73] \\
\hline $\begin{array}{l}167 \\
168\end{array}$ & $\begin{array}{l}\text { Sarcopodium sp. } \\
\text { FKJ-0025 }\end{array}$ & $\begin{array}{l}\text { Deep-sea } \\
200 \mathrm{~m}\end{array}$ & CT & $\begin{array}{l}\text { IC }_{50} 47 \mu \mathrm{g} / \mathrm{mL} \text { (Jurkat) } \\
\left.\text { IC }_{50} 37-66 \mu \mathrm{g} / \mathrm{mL} \text { (Jurkat, HL-60, Panc } 1\right)\end{array}$ & {$[169]$} \\
\hline 233 & $\begin{array}{l}\text { Cladosporium sp. } \\
\text { OUCMDZ-302 }\end{array}$ & Mangrove & CT & $\mathrm{IC}_{\mathbf{5 0}} \mathbf{1 0} \boldsymbol{\mu M}(\mathrm{H} 1975)$ & [116] \\
\hline 236 & $\begin{array}{l}\text { Penicillium chrysoge- } \\
\text { num MCCC } \\
\text { 3A00292 }\end{array}$ & $\begin{array}{l}\text { Deep-sea } \\
2076 \mathrm{~m}\end{array}$ & CT & $\mathrm{IC}_{\mathbf{5 0}} \mathbf{7 . 7 0 - 1 3 . 7 5} \boldsymbol{\mu M}(\mathrm{BIU}-87$, ECA109, BEL-7402) & {$[210]$} \\
\hline
\end{tabular}


Table 4. (Contd.)

\begin{tabular}{|c|c|c|c|c|c|}
\hline Cpd. & Producer/species & $\begin{array}{l}\text { Extremophile } \\
\text { type/origin }\end{array}$ & $\begin{array}{l}\text { Activity/ } \\
\text { toxicity }\end{array}$ & $\mathrm{EC}_{50}, \mathrm{IC}_{50}, \mathrm{MIC}$ & Ref. \\
\hline $\begin{array}{l}183 \\
184\end{array}$ & $\begin{array}{l}\text { Penicillium chrysoge- } \\
\text { num } \\
\text { MCCC 3A00292 }\end{array}$ & $\begin{array}{l}\text { Deep-sea } \\
2076 \mathrm{~m}\end{array}$ & $\begin{array}{l}\text { CT } \\
\text { AB } \\
\text { AF }\end{array}$ & 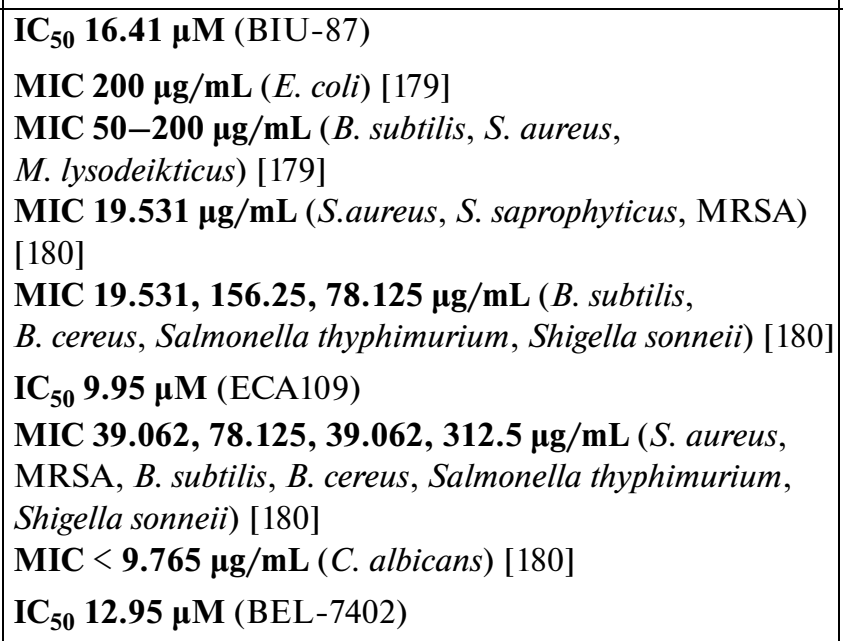 & [46] \\
\hline 182 & $\begin{array}{l}\text { Aspergillus sp. } \\
\text { Pleosporales sp. SK7 }\end{array}$ & $\begin{array}{l}\text { Marine } \\
\text { Mangrove }\end{array}$ & CT & IC $_{50}$ 30.2-45 $\boldsymbol{\mu g} / \mathbf{m L}($ HepG2, HL-60, MOLT-3) [182] & $\begin{array}{r}{[74]} \\
{[186]}\end{array}$ \\
\hline $\begin{array}{l}250 \\
251\end{array}$ & $\begin{array}{l}\text { Penicillium chrysoge- } \\
\text { num } \\
\text { MCCC 3A00292 }\end{array}$ & $\begin{array}{l}\text { Deep-sea } \\
2076 \mathrm{~m}\end{array}$ & CT & $\begin{array}{l}\text { IC }_{50} \mathbf{1 2 . 4 9} \boldsymbol{\mu M}(\mathrm{ECA} 109) \\
\mathrm{IC}_{\mathbf{5 0}} \mathbf{1 5 . 6} \boldsymbol{\mu M}(\mathrm{ECA109)}\end{array}$ & {$[46]$} \\
\hline $\begin{array}{l}122 \\
256\end{array}$ & $\begin{array}{l}\text { Penicillium sp. } \\
\text { SCSIO } 06720\end{array}$ & $\begin{array}{l}\text { Deep-sea } \\
4762 \mathrm{~m}\end{array}$ & $\begin{array}{l}\text { CT } \\
\mathrm{AF}\end{array}$ & 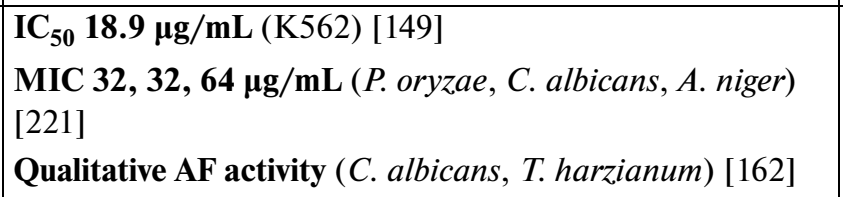 & [59] \\
\hline 181 & $\begin{array}{l}\text { Penicillium citrinum } \\
\text { HL-5126 }\end{array}$ & Mangrove & $\begin{array}{l}\mathrm{AB} \\
\mathrm{CT}\end{array}$ & $\mathrm{IC}_{50} 21.6 \mu \mathrm{g} / \mathrm{mL}(\mathrm{HeLa})$ & [124] \\
\hline $\begin{array}{l}221 \\
222\end{array}$ & $\begin{array}{l}\text { Penicillium citrinum } \\
\text { SCSIO } 41017\end{array}$ & Marine & $\begin{array}{l}\text { CT } \\
\text { AB } \\
\text { AV }\end{array}$ & $\begin{array}{l}\text { IC }_{\mathbf{5 0}} \mathbf{1 3 . 0 - 4 9 . 3} \boldsymbol{\mu M}(\mathrm{SF}-268, \mathrm{MCF}-7, \mathrm{HepG}-2, \mathrm{~A} 549) \\
\text { IC }_{\mathbf{5 0}} \mathbf{1 6 . 1}-\mathbf{5 5 . 4} \boldsymbol{\mu M}(\mathrm{SF}-268, \mathrm{MCF}-7, \mathrm{HepG}-2, \mathrm{~A} 549) \\
\text { MIC } 12.5 \boldsymbol{\mu g} / \mathbf{m L}(\text { B. cereus })[205] \\
\text { IC }_{\mathbf{5 0}} \mathbf{1 . 3}-\mathbf{4 7 . 9} \boldsymbol{\mu M}(\mathrm{SF}-268, \mathrm{MCF}-7, \mathrm{HepG}-2, \mathrm{~A} 549) \\
\text { IC }_{\mathbf{5 0}} \mathbf{1 6 . 4 1 - 1 1 5 . 3} \boldsymbol{\mu M}(\mathrm{SF}-268, \mathrm{MCF}-7, \mathrm{HepG}-2, \mathrm{~A} 549) \\
\text { IC }_{\mathbf{5 0}} \mathbf{1 4 . 5} \boldsymbol{\mu} \mathrm{M}(\mathrm{EV} 71)[206]\end{array}$ & [108] \\
\hline 137 & $\begin{array}{l}\text { Penicillium citrinum } \\
\text { SCSIO } 41017 \\
\text { Aspergillus sydowii } \\
\text { C1-S01-A7 }\end{array}$ & $\begin{array}{l}\text { Marine } \\
\text { Deep-sea } \\
4950 \mathrm{~m}\end{array}$ & $\mathrm{AB}$ & $\begin{array}{l}\text { MIC } 32.4 \mu \mathrm{g} / \mathrm{mL}(\mathrm{MR} S . \text { aureus ATCC } 43300, \mathrm{MR} \\
\text { S. aureus CGMCC) [77] }\end{array}$ & $\begin{array}{r}{[108]} \\
{[77]}\end{array}$ \\
\hline 186 & Pleosporales sp. SK7 & Mangrove & $\mathrm{CT}$ & $\mathrm{IC}_{50} 25.96 \mu \mathrm{M}(\mathrm{MDA}-\mathrm{MB}-435)$ & [186] \\
\hline 189 & $\begin{array}{l}\text { Aspergillus sp. } \\
\text { CUGB-F046 }\end{array}$ & Marine & $\begin{array}{l}\mathrm{AB} \\
\mathrm{CT} \\
\mathrm{AF}\end{array}$ & MIC $3.125 \mu \mathrm{g} / \mathrm{mL}$ (S. aureus, MRSA) & \\
\hline 187 & & & & 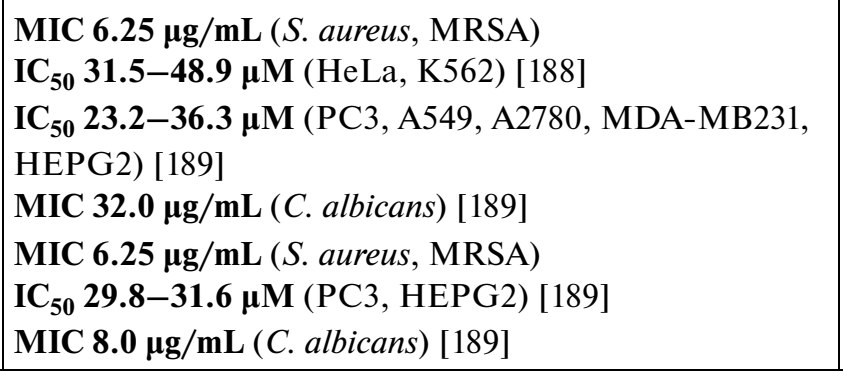 & [187] \\
\hline
\end{tabular}


<smiles>CC(=O)N[C@H](C(=O)O[C@H]1CCC(C)(C)[C@H]2C=CC3=C(COC3=O)[C@]12C)C(C)C</smiles>

Purpuride D

(458)

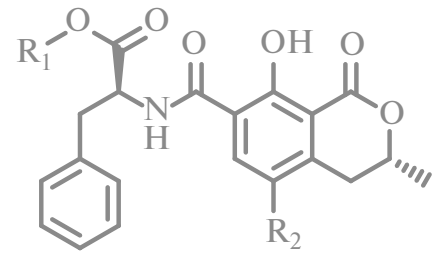

Ochratoxines A, B (459) $\mathrm{R}_{1}=\mathrm{H}, \mathrm{R}_{2}=\mathrm{CI}$ (460) $\mathrm{R}_{1}=\mathrm{H}, \mathrm{R}_{2}=\mathrm{H}$ Ochratoxin A ethyl ester (461) $\mathrm{R}_{1}=\mathrm{H}, \mathrm{R}_{2}=\mathrm{CI}$<smiles>[R]Oc1cc(C)cc(Oc2cc([R2])cc(C)c2)c1</smiles>

Trichoderolides A, B (462) $\mathrm{R}_{1}=\mathrm{R}_{2}=\mathrm{COCH}_{3}$ (463) $\mathrm{R}_{1}=\mathrm{COCH}_{2} \mathrm{CH}_{3}, \mathrm{R}_{2}=\mathrm{CH}_{3}$ (468) $\mathrm{R}_{1}=\mathrm{R}_{2}=\mathrm{CH}_{3}$

3-O-Methyldiorcinol (469) $\mathrm{R}_{1}=\mathrm{H}, \mathrm{R}_{2}=\mathrm{CH}_{3}$<smiles>CC(=O)O[C@H]1C[C@H](Cc2ccccc2)OC1=O</smiles>

Trichoderolide C (464)

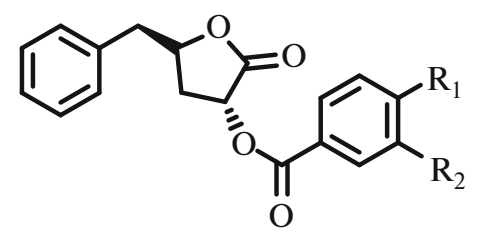

Trichoderolides D, E, F

(465) $\mathrm{R}_{1}=\mathrm{H}, \mathrm{R}_{2}=\mathrm{H}$

(466) $\mathrm{R}_{1}=\mathrm{OCH}_{3}, \mathrm{R}_{2}=\mathrm{H}$

(467) $\mathrm{R}_{1}=\mathrm{OCH}_{3}, \mathrm{R}_{2}=\mathrm{OCH}_{3}$

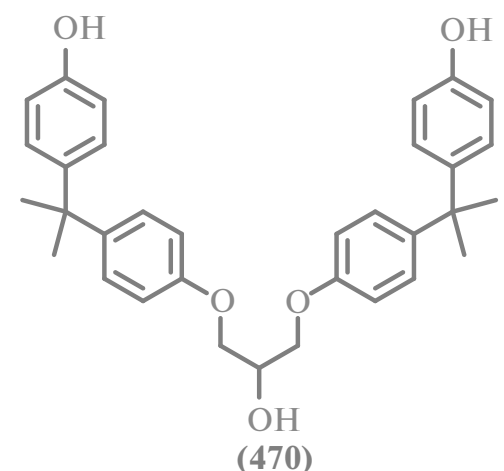

(470)

Fig. 29. Purpuride, ochratoxins, and trichoderolides.

Chemical study of mangrove endophyte Phyllosticta capitalensis [141] yielded two known compounds of mixed biosynthetic origin, i.e., meroterpenoids guignardones $A(\mathbf{4 7 4})$ and $\mathbf{J}(\mathbf{4 7 5})$, which showed antibiotic activity [309].

Two new furandion derivatives with moderate antifungal activity, asperfurandiones A and B $(\mathbf{4 7 6}, 477)$ were isolated from the marine strain of Aspergillus versicolor [310].

A new derivative of pentenoic acid, 1,1'-dioxin2,2'-propanoic acid (478) was obtained from the mangrove endophyte Cladosporium sp. JS 1-2 [121] and showed moderate antibacterial activity.

Talaromyces assiutensis JTY2 [311] of mangrove origin became the source of three new metabolites, i.e., the cyclopentenone derivative of talarocyclopenta A (479), the pyrogallol ester of talaracyclopent B $\mathbf{( 4 8 0 )}$, and the new itaconic acid derivative of talarocyclopenta $C(\mathbf{4 8 1})$, two of which $(\mathbf{4 7 9}, \mathbf{4 8 0})$ showed the antibacterial activity.

Three new lactones, penicilactones A-C (482484), were obtained from mangrove Penicillium sp. TGM112 [312]. Interestingly, only compound (482) of all the isomers showed antibacterial properties.

Two new pairs of epimeric acetaminophen derivatives of penicilqueis A-D (485-488), which showed promising activity against phytopathogenic fungi, were isolated from the Penicillium herquei JX4 culture [313].

Another new polycyclic cytotoxic metabolite, alternatone A (489) was isolated from the marine strain of the Alternaria genus [88].

Mangrove endophyte Cladosporium sp. JJM22 [314] was the source of two new metabolites $(495, \mathbf{4 9 6})$ and several known ones, only one of which (497) [315] showed the antibacterial activity.

The marine strain of Aspergillus terreus LGO13 [287] produces the terpenoids described above and several known metabolites with the antibiotic activity, i.e., butyrolactones I-III (490-492) [316] and aspulvinone $\mathrm{O}$ (498) [317]. A deep-sea micromycete of the same species, Aspergillus terreus SCSIO FZQ028 [318], became the source of several structurally similar butenolides including a new compound, $( \pm)$-asperteretal F (494), and its recently described close analog asperteretal E (493) [319] that contained 2-benzyl-3phenyl-substituted lactone. Another five known metabolites including the abovementioned butyrolactone III (492) were isolated. Antibacterial activity was shown in asperteretal E (493) and aspernolide A (499) [320].

Cultivation of marine fungus Aspergillus versicolor MCC 3A00080 [321] in the presence of the histone deacetylase inhibitor, vorinostat ( $N$-hydroxy- $N^{\prime}$ - 
Table 5. Data on the origin and biological activity of alkaloids

\begin{tabular}{|c|c|c|c|c|c|}
\hline Cpd. & Producer/species & $\begin{array}{l}\text { Extremophile } \\
\text { type/origin }\end{array}$ & $\begin{array}{l}\text { Activity/ } \\
\text { toxicity }\end{array}$ & $\mathrm{EC}_{50}, \mathrm{IC}_{50}, \mathrm{MIC}$ & Ref. \\
\hline 259 & Penicillium sp. ZZ380 & Marine & $\begin{array}{l}\mathrm{AB} \\
\mathrm{CT}\end{array}$ & $\begin{array}{l}\text { MIC } 5 \mu \mathrm{g} / \mathrm{mL}(\mathrm{MRSA}) \\
\text { IC }_{50} 11.32-29.1 \mu \mathrm{M}(\mathrm{U} 87-\mathrm{MG}, \mathrm{U} 251, \mathrm{SHG}-44, \mathrm{C} 6)\end{array}$ & [229] \\
\hline 258 & $\begin{array}{l}\text { Penicillium sp. ZZ380 } \\
\text { Penicillium erubescens } \\
\text { KUFA0220 }\end{array}$ & $\begin{array}{l}\text { Marine } \\
\text { Marine }\end{array}$ & $\begin{array}{l}\mathrm{AB} \\
\mathrm{CT}\end{array}$ & $\begin{array}{l}\text { MIC 8, 16, 32, 8, } 32 \boldsymbol{\mu g} / \mathbf{m L} \text { ( } \text { E. faecalis ATCC 29212, } \\
\text { E. faecium ATCC 19434, S. aureus ATCC 29213, } \\
\text { E. faecalis B3/101 (VRE), E. faecium } 1 / 6 / 63 \text { (VRE) [114] } \\
\text { MIC 48.35 } \boldsymbol{\mu M}\left(\text { M. tuberculosis } \mathrm{H}_{37} \mathrm{Ra}\right)[230] \\
\text { IC }_{\mathbf{5 0}} \mathbf{1 4 . 7 1 - 2 9 . 5 5} \boldsymbol{\mu M}(\mathrm{KB}, \mathrm{MCF}-7 \text {, Vero) [230] }\end{array}$ & $\begin{array}{l}{[229]} \\
{[114]}\end{array}$ \\
\hline 260 & Penicillium sp. ZZ380 & Marine & $\begin{array}{l}\mathrm{CT} \\
\mathrm{AB}\end{array}$ & 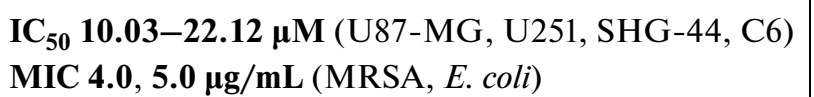 & \\
\hline 261 & & & & $\begin{array}{l}\mathrm{IC}_{50} \text { 9.95-22.39 } \boldsymbol{\mu M}(\mathrm{U} 87-\mathrm{MG}, \mathrm{U} 251, \mathrm{SHG}-44, \mathrm{C} 6) \\
\mathrm{MIC} 12.0,3.0 \mu \mathrm{g} / \mathrm{mL}(\mathrm{MRSA}, \text { E. coli) }\end{array}$ & \\
\hline 262 & & & & 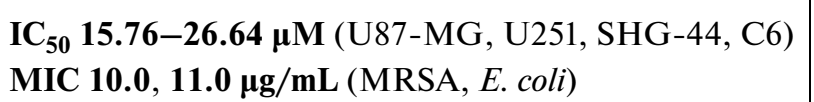 & \\
\hline 264 & & & & $\begin{array}{l}\mathrm{IC}_{50} 8.93-22.82 \mu \mathrm{M}(\mathrm{U} 87-\mathrm{MG}, \mathrm{U} 251, \mathrm{SHG}-44, \mathrm{C} 6) \\
\mathrm{MIC} 2.0,3.0 \mu \mathrm{g} / \mathrm{mL}(\mathrm{MRSA}, \text { E. coli) }\end{array}$ & [78] \\
\hline 266 & & & & $\mathrm{IC}_{\mathbf{5 0}} \mathbf{1 . 0 6}-\mathbf{8 . 5 2} \boldsymbol{\mu M}(\mathrm{U} 87-\mathrm{MG}, \mathrm{U} 251, \mathrm{SHG}-44, \mathrm{C} 6)$ & \\
\hline 267 & & & & $\begin{array}{l}\mathrm{IC}_{\mathbf{5 0}} \text { 12.89-23.92 } \boldsymbol{\mu M}(\mathrm{U} 87-\mathrm{MG}, \mathrm{U} 251, \mathrm{SHG}-44, \mathrm{C} 6) \\
\mathrm{MIC} 19.0, \mathbf{4 . 0} \boldsymbol{\mu g} / \mathrm{mL}(\mathrm{MRSA}, \text { E. coli })\end{array}$ & \\
\hline 268 & & & & $\begin{array}{l}\mathrm{IC}_{\mathbf{5 0}} \text { 7.44-19.18 } \boldsymbol{\mu \mathrm { M }}(\mathrm{U} 87-\mathrm{MG}, \mathrm{U} 251, \mathrm{SHG}-44, \mathrm{C} 6) \\
\mathrm{MIC} 4.0,2.0 \mu \mathrm{g} / \mathrm{mL}(\mathrm{MRSA}, \text { E. coli) }\end{array}$ & \\
\hline 292 & $\begin{array}{l}\text { Cocultivation } \\
\text { Aspergillus sulphureus } \\
\text { and Isaria felina }\end{array}$ & Marine & CT & $\mathrm{IC}_{\mathbf{5 0}} \mathbf{1 0 . 0} \boldsymbol{\mu} \mathrm{M}(22 \mathrm{Rv} 1)$ & [240] \\
\hline 299 & $\begin{array}{l}\text { Aspergillus alabamensis } \\
\text { EN-547 }\end{array}$ & Marine & $\mathrm{AB}$ & $\begin{array}{l}\text { MIC 64.0, 32.0, 32.0, } 64.0 \mu \mathrm{g} / \mathrm{mL} \text { (E. coli, Ed. ictaluri, } \\
\text { M. luteus, V. alginolyticus })\end{array}$ & \\
\hline 300 & & & & $\begin{array}{l}\text { MIC 16.0, 64.0, } 64.0 \mu \mathrm{g} / \mathrm{mL} \text { (E. coli, Ed. ictaluri, } \\
\text { M. luteus) }\end{array}$ & [234] \\
\hline 303 & & & & $\begin{array}{l}\text { MIC } 32,64,32,32 \mu \mathrm{g} / \mathrm{mL} \text { (E. coli, Ed. ictaluri, } \\
\text { M. luteus, V. alginolyticus) }\end{array}$ & \\
\hline
\end{tabular}


Table 5. (Contd.)

\begin{tabular}{|c|c|c|c|c|c|}
\hline Cpd. & Producer/species & $\begin{array}{l}\text { Extremophile } \\
\text { type/origin }\end{array}$ & $\begin{array}{l}\text { Activity/ } \\
\text { toxicity }\end{array}$ & $\mathrm{EC}_{50}, \mathrm{IC}_{50}, \mathrm{MIC}$ & Ref. \\
\hline 328 & $\begin{array}{l}\text { Aspergillus candidus } \\
\text { KUFA } 0062\end{array}$ & Marine & $\begin{array}{l}\text { CT } \\
\mathrm{AB} \\
\mathrm{AF}\end{array}$ & 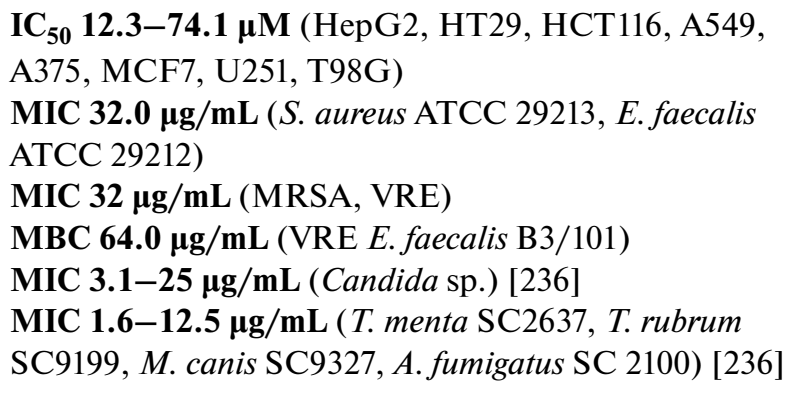 & [79] \\
\hline 329 & & & & $\begin{array}{l}\text { IC }_{\mathbf{5 0}} \mathbf{5 7 . 2 - 2 1 5 . 7} \boldsymbol{\mu M}(\text { HepG } 2, \text { HT29, HCT116, A549, } \\
\text { A375, MCF7, U251) }\end{array}$ & \\
\hline 296 & & & & $\begin{array}{l}\text { IC }_{\mathbf{5 0}} \mathbf{7 3 . 2 - 2 1 2 . 5} \boldsymbol{\mu M}(\mathrm{HepG} 2, \mathrm{HT} 29, \text { HCT116, A549, } \\
\text { A375, MCF7, U251) }\end{array}$ & \\
\hline 295 & & & & $\begin{array}{l}\text { IC }_{\mathbf{5 0}} \text { 34.8-94.8 } \boldsymbol{\mu M}(\mathrm{HepG} 2, \mathrm{HT} 29, \text { HCT } 116, \text { A549, } \\
\text { A375, MCF7) } \\
\text { IC }_{\mathbf{5 0}} 33.2 \boldsymbol{\mu g} / \mathbf{m L}(\mathrm{NS}-1 \text { ATCC TIB-18) }\end{array}$ & \\
\hline 294 & & & & IC $_{50} 28 \mu \mathrm{g} / \mathbf{m L}(\mathrm{NS}-1$ ATCC TIB-18) [235] & \\
\hline 297 & & & & IC $_{50} 43 \mu \mathrm{g} / \mathbf{m L}(\mathrm{NS}-1$ ATCC TIB-18) [235] & \\
\hline 298 & & & & $\begin{array}{l}\text { IC }_{\mathbf{5 0}} \mathbf{7 2 . 9 - 1 4 6 . 4} \boldsymbol{\mu M}(\text { HepG } 2, \text { HT29, HCT116, A549, } \\
\text { A375) } \\
\text { IC }_{\mathbf{5 0}} \mathbf{5 9} \boldsymbol{\mu g} / \mathbf{m L}(\mathrm{NS}-1 \text { ATCC TIB-18) [235] }\end{array}$ & \\
\hline 304 & & & & IC $_{50} 26 \mu \mathrm{g} / \mathrm{mL}(\mathrm{NS}-1$ ATCC TIB-18) [235] & \\
\hline 279 & Aspergillus sp. & Marine & CT & $\mathrm{IC}_{\mathbf{5 0}} \mathbf{4 . 8 6 - 6 9 . 4} \boldsymbol{\mu M}(\mathrm{PC}-3,22 \mathrm{Rv} 1, \mathrm{LNCaP})$ & {$[231]$} \\
\hline 291 & $\begin{array}{l}\text { Aspergillus sp. SCSIO } \\
\mathrm{XWS} 03 \mathrm{~F} 03\end{array}$ & Marine & $\mathrm{CT}$ & $\begin{array}{l}\mathrm{IC}_{50} 3.1 \mu \mathrm{M}(\mathrm{HL} 60) \\
\mathrm{IC}_{50} 4.9 \mu \mathrm{M}(\mathrm{LNCaP})\end{array}$ & {$[241]$} \\
\hline 331 & Cladosporium sp. JS1-2 & Mangrove & $\mathrm{AB}$ & MIC $12.5 \mu \mathrm{g} / \mathrm{mL}(S$. aureus $)$ & [121] \\
\hline 308 & Didymella sp. CYSK-4 & Mangrove & $\begin{array}{l}\text { CT } \\
\text { AT }\end{array}$ & $\begin{array}{l}\text { IC }_{\mathbf{5 0}} \text { 4.4-6.8 } \boldsymbol{\mu M} \text { (MDA-MB-435, MDA-MB-231, } \\
\text { SNB19, HCT116, NCI-H460, PC-3) }\end{array}$ & \\
\hline 309 & & & & $\begin{array}{l}\text { IC }_{\mathbf{5 0}} \mathbf{4 . 5}-\mathbf{2 0 . 0} \boldsymbol{\mu} \text { M (MDA-MB-435, MDA-MB-231, } \\
\text { SNB19, HCT116, NCI-H460, PC-3) }\end{array}$ & \\
\hline 310 & & & & $\begin{array}{l}\text { IC }_{\mathbf{5 0}} \mathbf{4 . 2}-\mathbf{7 . 8} \boldsymbol{\mu M}(\mathrm{MDA}-\mathrm{MB}-435, \mathrm{MDA}-\mathrm{MB}-231, \\
\mathrm{SNB} 19, \mathrm{HCT} 116, \mathrm{NCI}-\mathrm{H} 460, \mathrm{PC}-3) \\
\text { IC }_{\mathbf{5 0}} \mathbf{4 . 5}-\mathbf{6 . 6} \boldsymbol{\mu} \mathrm{M}(\mathrm{MDA}-\mathrm{MB}-231, \mathrm{HCT} 116)\end{array}$ & {$[244]$} \\
\hline 311 & & & & $\begin{array}{l}\text { IC }_{\mathbf{5 0}} \mathbf{1 2 - 2 9} \boldsymbol{\mu M}(\mathrm{MDA}-\mathrm{MB}-435, \mathrm{MDA}-\mathrm{MB}-231, \\
\mathrm{SNB} 19, \mathrm{HCT} 116, \mathrm{NCI}-\mathrm{H} 460, \mathrm{PC}-3) \\
\text { IC }_{\mathbf{5 0}} \mathbf{1 7 . 7} \boldsymbol{\mu} \mathrm{M}(\mathrm{Vero})[245] \\
\text { MIC } \mathbf{2 0 . 1 - 4 0 . 1} \boldsymbol{\mu g} / \mathbf{m L}(\mathrm{MABA}, \text { LORA) [245] }\end{array}$ & \\
\hline 312 & & & & $\begin{array}{l}\text { IC }_{50} 19.4 \boldsymbol{\mu M}(\text { Vero) }[245] \\
\text { MIC 5.2-13.4 } \boldsymbol{\mu g} / \mathbf{m L}(\text { MABA, LORA) [245] }\end{array}$ & \\
\hline $\begin{array}{l}314 \\
315\end{array}$ & $\begin{array}{l}\text { Cladosporium sp. } \\
\text { HNWSW-1 }\end{array}$ & Mangrove & CT & $\begin{array}{l}\text { IC }_{\mathbf{5 0}} \text { 25.6-41.7 } \boldsymbol{\mu M}(\mathrm{BEL}-7042, \mathrm{~K} 562, \mathrm{SGC}-7901) \\
\text { IC }_{\mathbf{5 0}} \mathbf{1 4 . 9 - 2 6 . 7} \boldsymbol{\mu M}(\text { Hela, BEL-7042) }\end{array}$ & {$[246]$} \\
\hline
\end{tabular}


Table 5. (Contd.)

\begin{tabular}{|c|c|c|c|c|c|}
\hline Cpd. & Producer/species & $\begin{array}{l}\text { Extremophile } \\
\text { type/origin }\end{array}$ & $\begin{array}{l}\text { Activity/ } \\
\text { toxicity }\end{array}$ & $\mathrm{EC}_{50}, \mathrm{IC}_{50}, \mathrm{MIC}$ & Ref. \\
\hline 269 & Tolypocladium sp. & $\begin{array}{l}\text { Mine soil, } \\
\text { Lianyuan, } \\
\text { China }\end{array}$ & $\begin{array}{l}\mathrm{AB} \\
\mathrm{CT}\end{array}$ & 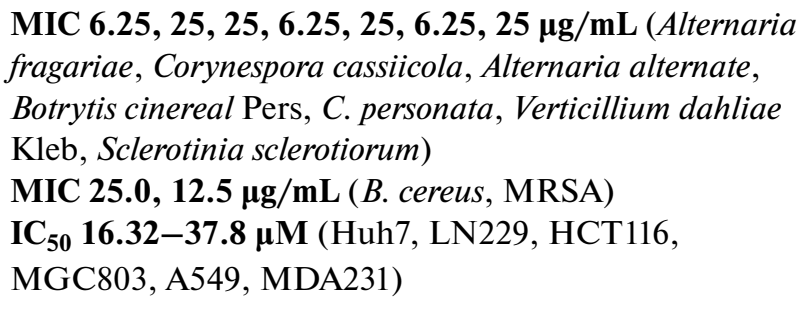 & \\
\hline 270 & & & & $\begin{array}{l}\text { MIC } 25, \mathbf{2 5}, \mathbf{5 0} \boldsymbol{\mu g} / \mathbf{m L}(\text { A. fragariae, B. cinereal Pers, } \\
\text { C. personata) } \\
\text { MIC } 50 \mu \mathrm{g} / \mathbf{m L}(\mathrm{MRSA}) \\
\mathrm{IC}_{\mathbf{5 0}} \mathbf{5 6 . 3 5}-\mathbf{1 2 8 . 5} \boldsymbol{\mu M}(\mathrm{Huh} 7, \mathrm{LN} 229, \mathrm{HCT} 116, \\
\text { MGC } 803, \text { A549, MDA231) }\end{array}$ & \\
\hline 271 & & & & 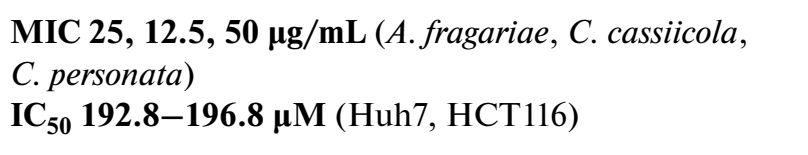 & \\
\hline 272 & & & & 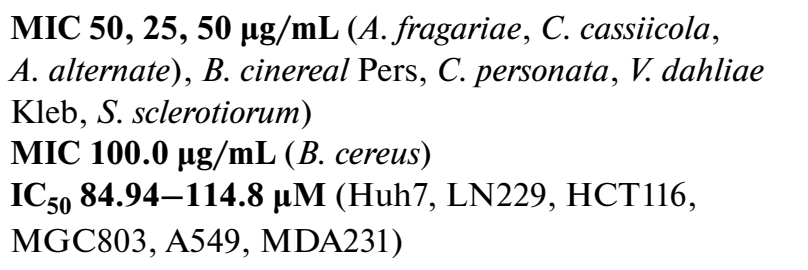 & \\
\hline 273 & & & & $\begin{array}{l}\text { MIC 6.25, 12.5, } 12.5 \mu \mathrm{g} / \mathrm{mL} \text { (A. fragariae, C. cassiicola, } \\
\text { C. personata) } \\
\mathrm{IC}_{\mathbf{5 0}} 198.6 \mu \mathrm{M}(\mathrm{A} 549)\end{array}$ & {$[232]$} \\
\hline 274 & & & & $\begin{array}{l}\text { MIC 25.0, } 12.5 \boldsymbol{\mu g} / \mathbf{m L}(\text { A. fragariae, } C \text {. cassiicola }) \\
\text { IC }_{\mathbf{5 0}} \mathbf{5 4 . 2 9 - 1 5 7 . 2} \boldsymbol{\mu M}(\text { Huh7, LN229, HCT116, } \\
\text { MGC803, A549, MDA231) }\end{array}$ & \\
\hline 275 & & & & $\begin{array}{l}\text { MIC 6.25, 50, 25, } 100 \mu \mathrm{g} / \mathrm{mL}(\text { A. fragariae, } \text { A. alternate, } \\
\text { B. cinereal Pers, C. personata) } \\
\text { IC }_{\mathbf{5 0}} \mathbf{3 9 . 9 4 - 1 5 4 . 1} \boldsymbol{\mu M}(\mathrm{Huh} 7, \mathrm{LN} 229, \mathrm{HCT} 116, \\
\text { MGC803, A549, MDA231) }\end{array}$ & \\
\hline 276 & & & & $\begin{array}{l}\text { MIC } 25.0 \mu \mathrm{g} / \mathrm{mL} \text { (C. personata), } \\
\text { MIC } 12,5 \mu \mathrm{g} / \mathrm{mL}(\text { B. cereus }) \\
\text { MIC } 25.0 \mu \mathrm{g} / \mathrm{mL}(\mathrm{MRSA}, \text { M. lysodeikticus, Bact. para- } \\
\text { typhosum, B. subtilis, E. aerogenes, S. typhi, P. vulgaris) } \\
\text { IC }_{\mathbf{5 0}} \mathbf{3 1 . 4 9 - 6 4 . 4 9} \boldsymbol{\mu} \text { M (Huh7, LN229, HCT116, } \\
\text { MGC803, A549, MDA231) }\end{array}$ & \\
\hline 277 & & & & $\begin{array}{l}\text { MIC } 50 \mu \mathrm{g} / \mathrm{mL} \text { ( } \text { A. fragariae }) \\
\text { MIC } 100 \boldsymbol{\mu g} / \mathbf{m L}(\text { B. cereus }) \\
\text { IC }_{\mathbf{5 0}} \mathbf{5 0 . 9 5 - 1 2 9 . 1} \boldsymbol{\mu M}(\text { Huh7, LN229, HCT116, } \\
\text { MGC } 803, \text { A549, MDA231) }\end{array}$ & \\
\hline 278 & & & & $\begin{array}{l}\text { MIC } 25 \mu \mathrm{g} / \mathrm{mL} \text { (A. fragariae) } \\
\text { IC }_{50} 174.2 \mu \mathrm{M}(\mathrm{A} 549)\end{array}$ & \\
\hline 332 & Aspergillus sydowii SP-1 & Antarctica & $\mathrm{AB}$ & $\begin{array}{l}\text { MIC 4.0, 8.0, 32.0, } 16.0 \mu \mathrm{g} / \mathrm{mL}(S . \text { epidermidis, } \\
\text { S. aureus, MRSA, MRSE) }\end{array}$ & {$[44]$} \\
\hline
\end{tabular}


Table 5. (Contd.)

\begin{tabular}{|c|c|c|c|c|c|}
\hline Cpd. & Producer/species & $\begin{array}{l}\text { Extremophile } \\
\text { type/origin }\end{array}$ & $\begin{array}{l}\text { Activity/ } \\
\text { toxicity }\end{array}$ & $\mathrm{EC}_{50}, \mathrm{IC}_{50}, \mathrm{MIC}$ & Ref. \\
\hline 325 & $\begin{array}{l}\text { Aspergillus sp. } \\
\text { YQ-13 } \\
\text { Aspergillus fumigatus }\end{array}$ & $\begin{array}{l}\text { Hydrothermal } \\
\text { vent sediment, } \\
\text { Kueishantao, } \\
\text { Taiwan/ } \\
\text { Thermophile } \\
\text { Marine }\end{array}$ & $\mathrm{AB}$ & $\begin{array}{l}\text { MIC 8.75, 5.42, 19.67, 1.33, 11.91 } \boldsymbol{\mu g} / \mathbf{m L}(P . \text { aerugi- } \\
\text { nosa, E. coli, } S . \text { aureus, B. subtilis, MRSA) } \\
\text { MIC 21.13, 2.51, 3.72, 0.89, 6.25 } \boldsymbol{\mu g} / \mathbf{m L}(P . \text { aerugi- } \\
\text { nosa, E. coli, } S . \text { aureus, B. subtilis, MRSA) } \\
\text { IC }_{\mathbf{5 0}} \text { 21.0-28.0 } \boldsymbol{\mu M}(\mathrm{SW} 1116, \mathrm{~K} 562)[250]\end{array}$ & $\begin{array}{r}{[126]} \\
{[75]}\end{array}$ \\
\hline $\begin{array}{l}284 \\
286\end{array}$ & $\begin{array}{l}\text { Aspergillus fumigatus } \\
\text { SCSIO } 41012\end{array}$ & $\begin{array}{l}\text { Deep-sea } \\
3614 \mathrm{~m}\end{array}$ & $\begin{array}{l}\mathrm{AB} \\
\mathrm{AF}\end{array}$ & 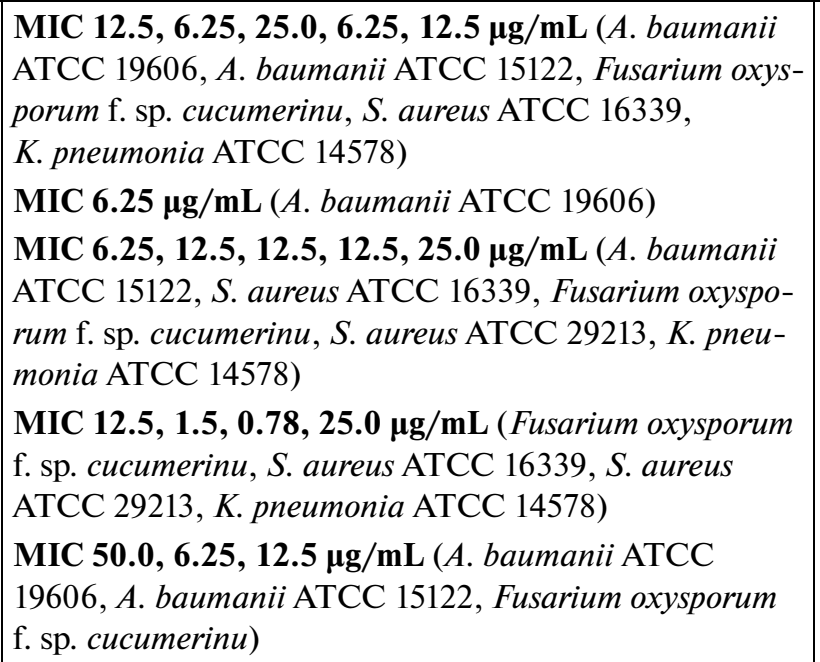 & [237] \\
\hline $\begin{array}{l}321 \\
324\end{array}$ & $\begin{array}{l}\text { Arthrinium sp. } \\
\text { UJNMF0008 }\end{array}$ & $\begin{array}{l}\text { Deep-sea } \\
3858 \mathrm{~m}\end{array}$ & $\begin{array}{l}\mathrm{AB} \\
\mathrm{CT}\end{array}$ & 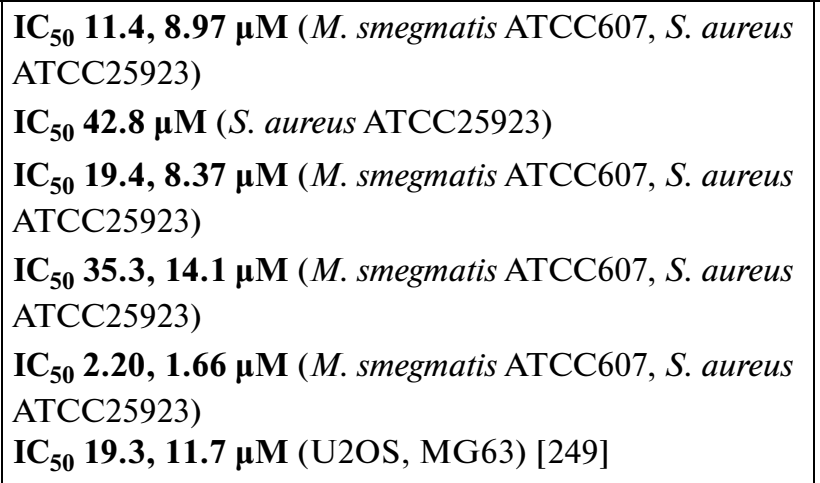 & [248] \\
\hline 330 & $\begin{array}{l}\text { Penicillium pinophilum } \\
\text { SCAU037 }\end{array}$ & Mangrove & $\mathrm{CT}$ & 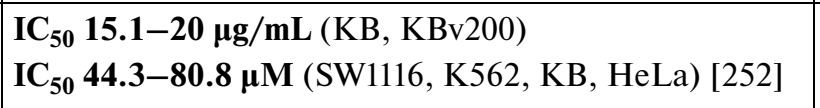 & {$[130]$} \\
\hline 333 & $\begin{array}{l}\text { Aspergillus sp. } \\
\text { SCSIO41211 }\end{array}$ & Mangrove & $\mathrm{CT}$ & $\begin{array}{l}\text { IC }_{\mathbf{5 0}} \mathbf{0 . 1 5}-\mathbf{2 2 . 9} \boldsymbol{\mu M}(\mathrm{H} 1975, \mathrm{U} 937, \mathrm{~K} 562, \mathrm{BGC} 823, \\
\text { MOLT-4, MCF-7, A549, HL60, Huh-7) }\end{array}$ & [254] \\
\hline $\begin{array}{l}305 \\
306\end{array}$ & $\begin{array}{l}\text { Eupenicillium sp. } \\
\text { HJ002 }\end{array}$ & Mangrove & $\mathrm{CT}$ & $\begin{array}{l}\text { IC }_{\mathbf{5 0}} \mathbf{1 . 5}-\mathbf{2 3 . 3} \boldsymbol{\mu} \mathbf{M}(\mathrm{A} 549, \mathrm{HeLa}, \mathrm{HepG}-2) \\
\text { IC }_{\mathbf{5 0}} \text { 18.6-47.2 } \boldsymbol{\mu M}(\mathrm{A} 549, \mathrm{HeLa}, \mathrm{HepG}-2)\end{array}$ & [242] \\
\hline 302 & Aspergillus versicolor & Mangrove & $\mathrm{CT}$ & $\mathrm{IC}_{50} 43.7 \boldsymbol{\mu M}(\mathrm{HeLa})$ & [243] \\
\hline
\end{tabular}

phenyloctanediamide), induced the biosynthesis of a new secondary metabolite of versiperol A (513), which showed weak antibacterial activity.

Three new derivatives of itaconic acid isolated from marine Aspergillus niger were named asperitaconic acids A-C (500-502) [322]. The described com- pounds did not show noticeable cytotoxicity but demonstrated a moderate antibacterial activity.

A new secondary metabolite, 1-(2',6'-dimethylphenyl)-2- $n$-propyl-1,2-dihydropyridazine-3,6-dione (512), which is the derivative of maleic hydrazide, was isolated from mangrove fungus Aspergillus sp. AV-2 
Table 6. Data on the origin and biological activity of terpenoids and related compounds

\begin{tabular}{|c|c|c|c|c|c|}
\hline Cpd. & Producer/species & $\begin{array}{l}\text { Extremophile } \\
\text { type/origin }\end{array}$ & $\begin{array}{l}\text { Activity/ } \\
\text { toxicity }\end{array}$ & $\mathrm{EC}_{50}, \mathrm{IC}_{50}, \mathrm{MIC}$ & Ref. \\
\hline 454 & $\begin{array}{l}\text { Aspergillus sydowii } \\
\text { C1-S01-A7 }\end{array}$ & Deep-sea $4950 \mathrm{~m}$ & $\mathrm{CT}$ & IC $_{\mathbf{5 0}} \mathbf{5 0} \boldsymbol{\mu g} / \mathbf{m L}($ HepG 2$)$ & [77] \\
\hline $\begin{array}{l}421 \\
435\end{array}$ & $\begin{array}{l}\text { Aspergillus fumigatus } \\
\text { SCSIO } 41012\end{array}$ & Deep-sea $3614 \mathrm{~m}$ & $\begin{array}{l}\mathrm{AB} \\
\mathrm{AF}\end{array}$ & $\begin{array}{l}\text { MIC } 50 \mu \mathrm{g} / \mathrm{mL} \text { (A. baumanii ATCC 19606) } \\
\text { MIC } 12.5,3.125 \mu \mathrm{g} / \mathrm{mL} \text { (A. baumanii ATCC } \\
15122, \text { K. pneumonia ATCC 14578) } \\
\text { MIC } 1.5 \mu \mathrm{g} / \mathrm{mL} \text { (F. oxysporum f. sp. Momordicae) } \\
\text { MIC } 100 \mu \mathrm{g} / \mathrm{mL} \text { (S. epidermidis) [281] } \\
\text { MIC } 100 \mu \mathrm{g} / \mathrm{mL} \text { (Enterococcus durans) [281] }\end{array}$ & [237] \\
\hline 424 & $\begin{array}{l}\text { Aspergillus fumigatus } \\
\text { SCSIO } 41012 \\
\text { Aspergillus fumigatus } \\
\text { HNMF0047 }\end{array}$ & $\begin{array}{l}\text { Deep-sea } 3614 \mathrm{~m} \\
\text { Marine }\end{array}$ & $\mathrm{AB}$ & 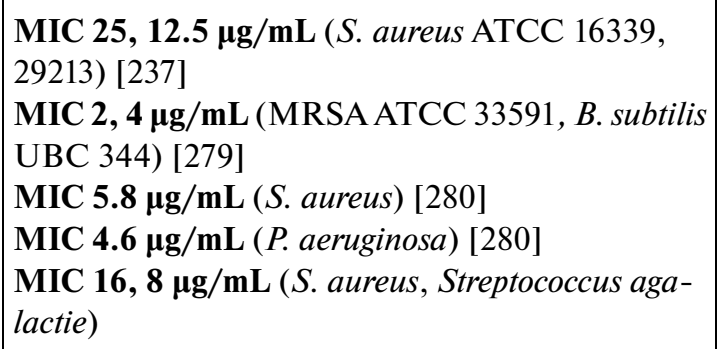 & $\begin{array}{l}{[237]} \\
{[282]}\end{array}$ \\
\hline $\begin{array}{l}422 \\
423\end{array}$ & $\begin{array}{l}\text { Aspergillus fumigatus } \\
\text { HNMF0047 }\end{array}$ & Marine & $\mathrm{AB}$ & $\begin{array}{l}\text { MIC } 16 \mu \mathrm{g} / \mathrm{mL}(S . \text { aureus, } S \text {. agalactie }) \\
\text { MIC } 8,2 \mu \mathrm{g} / \mathrm{mL} \text { (S. aureus, } S \text {. agalactie })\end{array}$ & [282] \\
\hline $\begin{array}{l}395 \\
397\end{array}$ & Stachybotrys chartarum & Marine & $\begin{array}{l}\mathrm{AB} \\
\mathrm{AF}\end{array}$ & $\begin{array}{l}\text { MIC } 16 \mu \mathrm{g} / \mathrm{mL} \text { (A. baumanii) } \\
\text { MIC } 16 \mu \mathrm{g} / \mathrm{mL} \text { (E. faecalis) } \\
\text { MIC } 32 \mu \mathrm{g} / \mathrm{mL} \text { (MRSA) } \\
\text { MIC } 8 \mu \mathrm{g} / \mathrm{mL} \text { (C. albicans) }\end{array}$ & {$[271]$} \\
\hline 400 & Aspergillus porosus & Marine & $\begin{array}{l}\mathrm{AB} \\
\mathrm{CT} \\
\mathrm{AF}\end{array}$ & 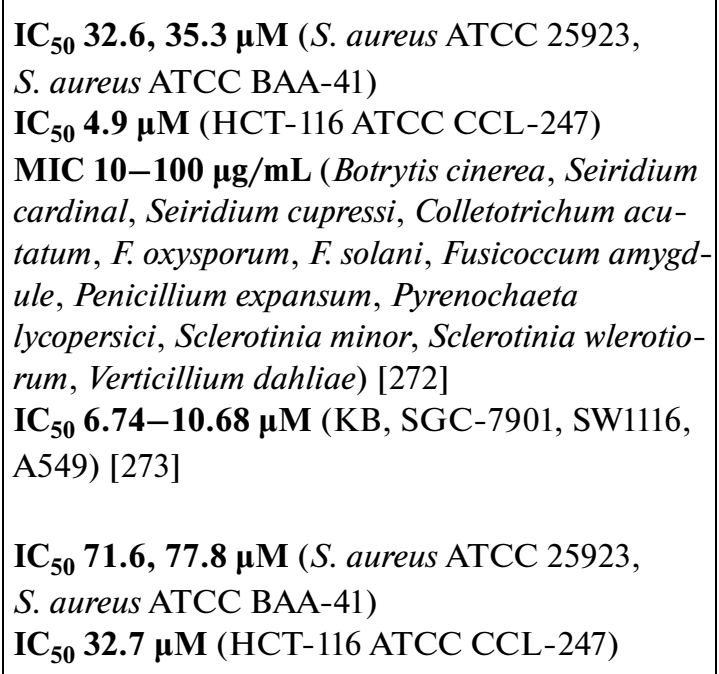 & [209] \\
\hline 431 & $\begin{array}{l}\text { Aspergillus alabamensis } \\
\text { EN-547 }\end{array}$ & Marine & $\mathrm{AB}$ & MIC 32, $64 \mu \mathrm{g} / \mathrm{mL}$ (Ed. ictaluri, V. alginolyticus) & {$[234]$} \\
\hline
\end{tabular}


Table 6. (Contd.)

\begin{tabular}{|c|c|c|c|c|c|}
\hline Cpd. & Producer/species & $\begin{array}{l}\text { Extremophile } \\
\text { type/origin }\end{array}$ & $\begin{array}{l}\text { Activity/ } \\
\text { toxicity }\end{array}$ & $\mathrm{EC}_{50}, \mathrm{IC}_{50}, \mathrm{MIC}$ & Ref. \\
\hline 338 & \multirow[t]{9}{*}{ Aspergillus flocculosus } & \multirow[t]{9}{*}{ Marine } & \multirow[t]{9}{*}{$\mathrm{CT}$} & $\begin{array}{l}\text { IC }_{\mathbf{5 0}} \mathbf{0 . 1 4 - 0 . 2 4} \boldsymbol{\mu M}(\mathrm{HCT}-15, \mathrm{NUGC}-3, \mathrm{NCI}- \\
\mathrm{H} 23, \mathrm{ACHN}, \mathrm{PC}-3, \text { MDA-MB-231) }\end{array}$ & \multirow{9}{*}[255]{} \\
\hline 339 & & & & $\begin{array}{l}\text { IC }_{\mathbf{5 0}} \mathbf{0 . 4 4 - 0 . 6 3} \boldsymbol{\mu} \text { M (HCT-15, NUGC-3, NCI- } \\
\text { H23, ACHN, PC-3, MDA-MB-231) }\end{array}$ & \\
\hline 340 & & & & $\begin{array}{l}\text { IC }_{\mathbf{5 0}} \mathbf{0 . 8 8}-\mathbf{1 . 4 0} \boldsymbol{\mu} \mathbf{M}(\mathrm{HCT}-15, \mathrm{NUGC}-3, \mathrm{NCI}- \\
\mathrm{H} 23, \mathrm{ACHN}, \mathrm{PC}-3, \text { MDA-MB-231) }\end{array}$ & \\
\hline 341 & & & & $\begin{array}{l}\mathbf{I C}_{\mathbf{5 0}} \mathbf{1 . 0 7 - 1 . 5 0} \boldsymbol{\mu} \mathbf{M}(\mathrm{HCT}-15, \text { NUGC-3, NCI- } \\
\mathrm{H} 23, \text { ACHN, PC-3, MDA-MB-231) }\end{array}$ & \\
\hline 342 & & & & $\begin{array}{l}\text { IC }_{\mathbf{5 0}} \text { 1.53-2.01 } \boldsymbol{\mu} \text { M (HCT-15, NUGC-3, NCI- } \\
\mathrm{H} 23, \text { ACHN, PC-3, MDA-MB-231) }\end{array}$ & \\
\hline 345 & & & & $\begin{array}{l}\text { IC }_{\mathbf{5 0}} \mathbf{0 . 1 9 - 0 . 4 3} \boldsymbol{\mu} \mathbf{M}(\mathrm{HCT}-15, \text { NUGC-3, NCI- } \\
\mathrm{H} 23, \text { ACHN, PC-3, MDA-MB-231) }\end{array}$ & \\
\hline 346 & & & & $\begin{array}{l}\text { IC }_{\mathbf{5 0}} \mathbf{0 . 1 6}-\mathbf{0 . 3 6} \boldsymbol{\mu} \mathbf{M}(\mathrm{HCT}-15, \text { NUGC-3, NCI- } \\
\mathrm{H} 23, \mathrm{ACHN}, \mathrm{PC}-3, \mathrm{MDA}-\mathrm{MB}-231) \\
\text { IC }_{\mathbf{5 0}} \mathbf{8} \boldsymbol{\mu} \mathbf{M}(\mathrm{CLL})[256]\end{array}$ & \\
\hline 343 & & & & $\begin{array}{l}\text { IC }_{\mathbf{5 0}} \mathbf{0 . 2 0 - 0 . 3 0} \boldsymbol{\mu} \mathbf{M}(\mathrm{HCT}-15, \text { NUGC-3, NCI- } \\
\mathrm{H} 23, \mathrm{ACHN}, \mathrm{PC}-3, \text { MDA-MB-231) }\end{array}$ & \\
\hline 344 & & & & $\begin{array}{l}\text { IC }_{\mathbf{5 0}} \mathbf{0 . 1 9 - 0 . 4 2} \boldsymbol{\mu} \mathbf{M}(\mathrm{HCT}-15, \text { NUGC-3, NCI- } \\
\text { H23, ACHN, PC-3, MDA-MB-231) }\end{array}$ & \\
\hline 389 & \multirow[t]{3}{*}{ Talaromyces purpurogenus } & \multirow[t]{3}{*}{ Marine } & \multirow[t]{3}{*}{ CT } & 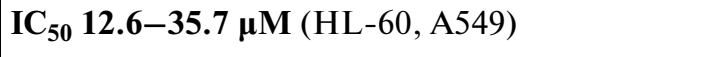 & \multirow[b]{3}{*}[54]{} \\
\hline 404 & & & & $\begin{array}{l}\mathrm{IC}_{\mathbf{5 0}} \text { 6.5-25.8 } \boldsymbol{\mu} \mathrm{M}(\mathrm{SW} 480, \mathrm{HL}-60, \mathrm{~A} 549, \\
\mathrm{MCF}-7)\end{array}$ & \\
\hline 405 & & & & $\begin{array}{l}\text { IC }_{\mathbf{5 0}} \mathbf{7 . 9 - 2 3 . 8} \boldsymbol{\mu M}(\mathrm{SW} 480, \mathrm{HL}-60, \mathrm{~A} 549, \\
\mathrm{MCF}-7) \\
\mathbf{E D}_{\mathbf{5 0}} \mathbf{2 . 2} \boldsymbol{\mu \mathrm { g }} / \mathbf{m L}(\mathrm{P}-388) 10.1039 / \mathrm{a} 903840 \mathrm{j} \\
\mathbf{G I}_{\mathbf{5 0}} \mathbf{1 . 8}-\mathbf{1 5 . 8} \boldsymbol{\mu M}(38 \text { Human cancer cell lines }) \\
{[269]}\end{array}$ & \\
\hline 402 & Penicillium sp. TJ403-1 & Marine & $\mathrm{CT}$ & $\begin{array}{l}\text { IC }_{\mathbf{5 0}} \text { 4.92-8.6 } \boldsymbol{\mu M}(\mathrm{HL}-60, \mathrm{~A} 549, \mathrm{HEP} 3 \mathrm{~B}) \\
\text { IC }_{\mathbf{5 0}} \mathbf{7 . 4 4 - 3 2 . 5} \boldsymbol{\mu M}(\mathrm{MCF}-7, \mathrm{~A} 549)[275]\end{array}$ & {$[51]$} \\
\hline 351 & \multirow{6}{*}{$\begin{array}{l}\text { Aspergillus ochraceus } \\
\text { Jcma1F17 }\end{array}$} & \multirow[t]{6}{*}{ Marine } & \multirow{6}{*}{$\begin{array}{l}\mathrm{CT} \\
\mathrm{AV}\end{array}$} & IC $_{50}$ 23-30 $\boldsymbol{\mu M}(\mathrm{ACHN}, \mathrm{OS}-\mathrm{RS}-2,786-\mathrm{O})$ & \multirow{6}{*}{ [257] } \\
\hline 349 & & & & & \\
\hline 352 & & & & $\mathrm{IC}_{\mathbf{5 0}}$ 11-14 $\boldsymbol{\mu M}(\mathrm{ACHN}, \mathrm{OS}-\mathrm{RS}-2,786-\mathrm{O})$ & \\
\hline 350 & & & & $\begin{array}{l}\text { IC }_{\mathbf{5 0}} \mathbf{0 . 8 9}-\mathbf{1 . 5} \boldsymbol{\mu M}(\mathrm{ACHN}, \mathrm{OS}-\mathrm{RS}-2, \mathbf{7 8 6 - O}) \\
\text { IC }_{\mathbf{5 0}} \mathbf{2 . 1 1 - 6 . 3 5} \boldsymbol{\mu M}(\mathrm{H} 1975, \mathrm{U} 937, \mathrm{~K} 562, \\
\mathrm{BGC} 823, \text { Molt-4, MCF-7, A549, HeLa, HL60, } \\
\text { Huh-7) [258] }\end{array}$ & \\
\hline 353 & & & & $\begin{array}{l}\text { IC }_{\mathbf{5 0}} \mathbf{4 . 3 - 1 1} \boldsymbol{\mu M}(\mathrm{ACHN}, \mathrm{OS}-\mathrm{RS}-2,786-\mathrm{O}) \\
\text { IC }_{\mathbf{5 0}} \mathbf{1 . 9 5 - 1 7} \boldsymbol{\mu M}(\mathrm{H} 1975, \mathrm{U} 937, \mathrm{~K} 562, \\
\text { BGC } 823, \text { Molt-4, MCF-7, A549, HeLa, HL60, }_{\text {Huh-7) }} \\
\text { IC }_{\mathbf{5 0}} \mathbf{1 7}, \mathbf{9 . 4} \boldsymbol{\mu M}(\mathrm{H} 3 \mathrm{~N} 2, \text { EV71) [258] }\end{array}$ & \\
\hline 354 & & & & IC $_{\mathbf{5 0}} \mathbf{2 0 - 3 0} \boldsymbol{\mu M}(\mathrm{ACHN}, \mathrm{OS}-\mathrm{RS}-2,786-\mathrm{O})$ & \\
\hline
\end{tabular}


Table 6. (Contd.)

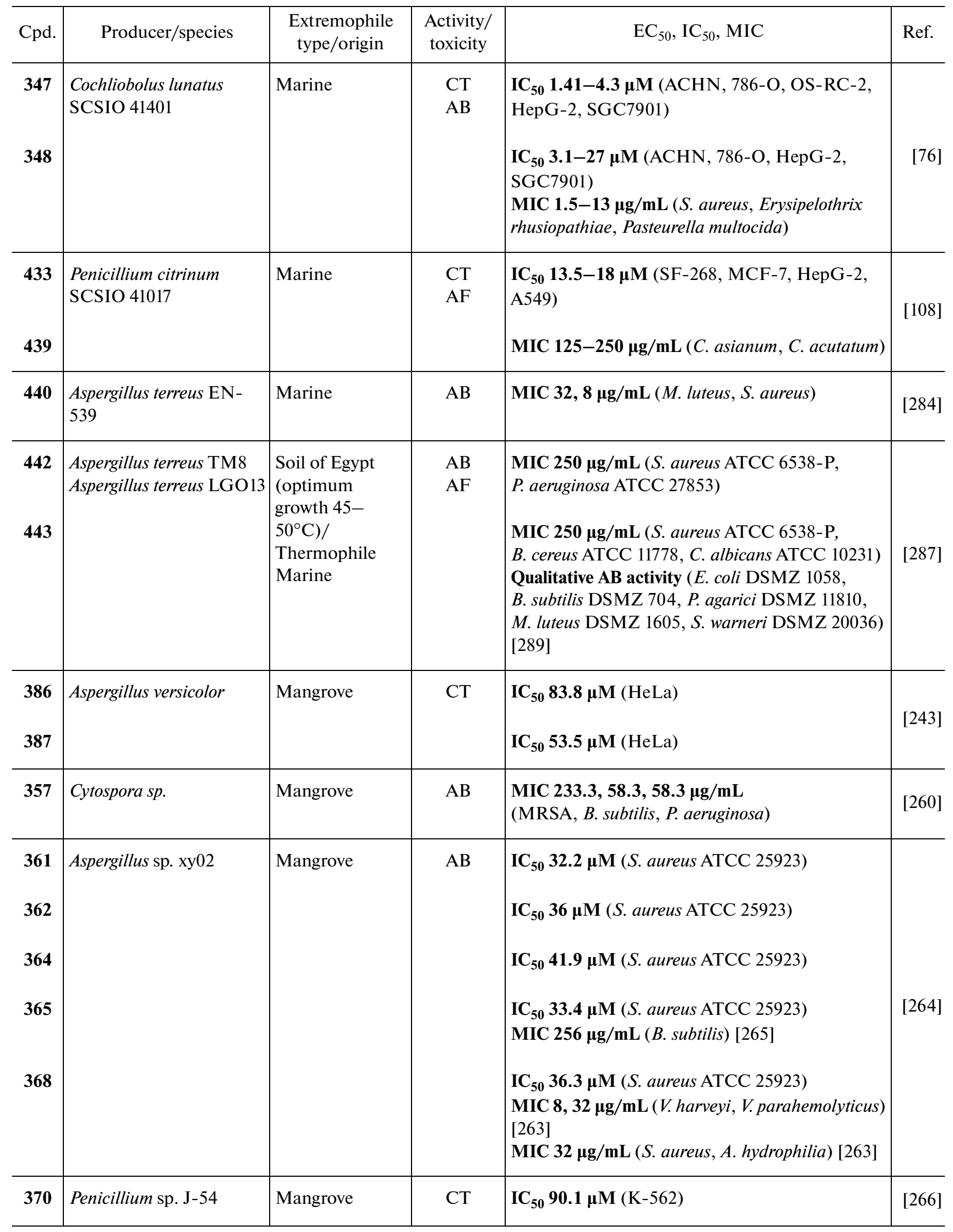


Table 6. (Contd.)

\begin{tabular}{|c|c|c|c|c|c|}
\hline Cpd. & Producer/species & $\begin{array}{l}\text { Extremophile } \\
\text { type/origin }\end{array}$ & $\begin{array}{l}\text { Activity/ } \\
\text { toxicity }\end{array}$ & $\mathrm{EC}_{50}, \mathrm{IC}_{50}, \mathrm{MIC}$ & Ref. \\
\hline 410 & \multirow[t]{9}{*}{ Eutypella sp. D-1 } & \multirow[t]{9}{*}{ Arctic } & \multirow[t]{9}{*}{ CT } & $\begin{array}{l}\mathbf{I C}_{50} \text { 2.2-6 } \boldsymbol{\mu M}(\mathrm{HeLa}, \mathrm{MCF}-7, \mathrm{HCT}-116, \\
\text { PANC-1, SW1990) }\end{array}$ & \multirow{9}{*}[276]{} \\
\hline 411 & & & & $\begin{array}{l}\text { IC }_{\mathbf{5 0}} \text { 1-3.6 } \boldsymbol{\mu M}(\mathrm{HeLa}, \mathrm{MCF}-7, \mathrm{HCT}-116 \text {, } \\
\text { PANC-1, SW1990) }\end{array}$ & \\
\hline 412 & & & & $\begin{array}{l}\text { IC }_{\mathbf{5 0}} \mathbf{0 . 8}-\mathbf{2 . 1} \boldsymbol{\mu M}(\mathrm{HeLa}, \mathrm{MCF}-7, \mathrm{HCT}-116, \\
\text { PANC-1, SW1990) }\end{array}$ & \\
\hline 413 & & & & $\mathrm{IC}_{50} 26.6 \mu \mathrm{M}(\mathrm{PANC}-1)$ & \\
\hline 414 & & & & IC $_{50} 29.4(\mathrm{HCT}-116)$ & \\
\hline 406 & & & & $\begin{array}{l}\text { IC }_{\mathbf{5 0}} \mathbf{1 0 . 3 - 1 3 . 1} \boldsymbol{\mu M}(\mathrm{HeLa}, \mathrm{MCF}-7, \mathrm{HCT}-116, \\
\text { PANC-1, SW1990) }\end{array}$ & \\
\hline 407 & & & & $\begin{array}{l}\text { IC }_{\mathbf{5 0}} \text { 6-8.4 } \boldsymbol{\mu M}(\mathrm{HeLa}, \mathrm{MCF}-7, \mathrm{HCT}-116 \\
\text { PANC-1, SW1990) }\end{array}$ & \\
\hline 408 & & & & $\begin{array}{l}\text { IC }_{\mathbf{5 0}} \mathbf{0 . 3}-\mathbf{1 . 8} \boldsymbol{\mu} \mathbf{M}(\mathrm{HeLa}, \mathrm{MCF}-7, \mathrm{HCT}-116 \\
\text { PANC-1, SW1990) }\end{array}$ & \\
\hline 409 & & & & $\begin{array}{l}\text { IC }_{\mathbf{5 0}} \text { 3.31-44.1 } \boldsymbol{\mu M}(\mathrm{U} 251, \mathrm{SW}-1990, \mathrm{SG} 7901 \text {, } \\
\mathrm{MCF}-7, \mathrm{Huh}-7, \mathrm{HeLa}, \mathrm{H} 460)\end{array}$ & \\
\hline 358 & $\begin{array}{l}\text { Aspergillus sydowii } \mathrm{SP}-1 \\
\text { Aspergillus sp. } \mathrm{xy} 02\end{array}$ & $\begin{array}{l}\text { Antarctica } \\
\text { Mangrove }\end{array}$ & $\begin{array}{l}\mathrm{AB} \\
\mathrm{AF}\end{array}$ & $\begin{array}{l}\text { MIC } 0.5,1,0.25,0.5 \mu \mathrm{g} / \mathrm{mL}(S . \text { aureus, MRSA, } \\
\text { S. epidermidis, MRSE) }\end{array}$ & \\
\hline 359 & & & & $\begin{array}{l}\text { MIC } 0.5, \mathbf{1}, \mathbf{0 . 2 5}, \mathbf{0 . 5} \boldsymbol{\mu g} / \mathbf{m L}(S . \text { aureus, MRSA, } \\
\text { S. epidermidis, MRSE) [44] } \\
\text { IC }_{\mathbf{5 0}} \mathbf{3 4} \boldsymbol{\mu M}(\text { S. aureus ATCC 25923) [264] } \\
\text { MIC } 0.5 \mu \mathrm{g} / \mathbf{m L} \text { (S. aureus, V. parahemolyticus) } \\
\text { [263] } \\
\text { MIC } 32 \boldsymbol{\mu g} / \mathbf{m L} \text { (Gaeumannomyces graminis) } \\
\text { [263] }\end{array}$ & $\begin{array}{r}{[44]} \\
{[264]}\end{array}$ \\
\hline 446 & $\begin{array}{l}\text { Penicillium granulatum } \\
\text { MCCC } 3 \mathrm{~A} 00475\end{array}$ & Deep-sea $2284 \mathrm{~m}$ & $\begin{array}{l}\text { CT } \\
\text { AF }\end{array}$ & 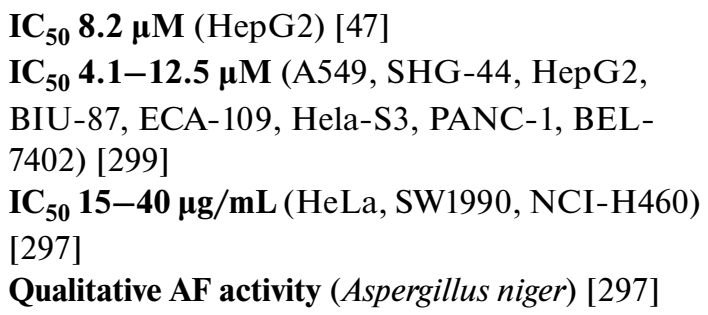 & $\begin{array}{r}{[47]} \\
{[299]}\end{array}$ \\
\hline $\begin{array}{r}453 \\
445\end{array}$ & $\begin{array}{l}\text { Penicillium granulatum } \\
\text { MCCC 3A00475 }\end{array}$ & Deep-sea $2284 \mathrm{~m}$ & CT & $\begin{array}{l}\mathbf{I C}_{\mathbf{5 0}} \mathbf{8 . 6} \boldsymbol{\mu M}(\mathrm{HepG}) \\
\mathbf{I C}_{\mathbf{5 0}} \mathbf{1 . 2} \boldsymbol{\mu M}(\mathrm{DLD}-1 \text { cells on polyHEMA- } \\
\text { coated) [298] } \\
\mathbf{I C}_{\mathbf{5 0}} \mathbf{4 0} \boldsymbol{\mu} \mathbf{M}(\mathrm{DLD}-1 \text { cells on uncoated plates) } \\
{[298]}\end{array}$ & [47] \\
\hline
\end{tabular}


Table 6. (Contd.)

\begin{tabular}{|c|c|c|c|c|c|}
\hline Cpd. & Producer/species & $\begin{array}{l}\text { Extremophile } \\
\text { type/origin }\end{array}$ & $\begin{array}{l}\text { Activity/ } \\
\text { toxicity }\end{array}$ & $\mathrm{EC}_{50}, \mathrm{IC}_{50}, \mathrm{MIC}$ & Ref. \\
\hline $\begin{array}{r}452 \\
447\end{array}$ & $\begin{array}{l}\text { Penicillium granulatum } \\
\text { MCCC 3A00475 }\end{array}$ & Deep-sea $2284 \mathrm{~m}$ & $\mathrm{CT}$ & $\begin{array}{l}\text { IC }_{\mathbf{5 0}} \text { 5.5-8.3 } \boldsymbol{\mu M}(\mathrm{A} 549, \mathrm{SHG}-44) \\
\text { IC }_{\mathbf{5 0}} \text { 4.8-14.4 } \boldsymbol{\mu M} \text { (A549, SHG-44, HepG2, } \\
\text { BIU-87, ECA-109, Hela-S3, PANC-1, BEL- } \\
\text { 7402) } \\
\text { IC }_{\mathbf{5 0}} \text { 4.4-9.2 } \boldsymbol{\mu M} \text { (A549, HepG2, BIU-87, ECA- } \\
\text { 109, Hela-S3) } \\
\text { IC }_{\mathbf{5 0}} \text { 4.4-9.9 } \boldsymbol{\mu M} \text { (A549, SHG-44, ECA-109, } \\
\text { Hela-S3, PANC-1) } \\
\text { Qualitative AF activity (C. albicans ATCC 10231, } \\
\text { S. cerevisiae ATCC 9080) [300] } \\
\text { Qualitative AB activity (S. aureus ATCC 6538-P, } \\
\text { B. cereus ATCC 11778, B. subtilis ATCC 6633) } \\
\text { [300] }\end{array}$ & [299] \\
\hline 385 & Penicillium griseofulvum & Deep-sea $1420 \mathrm{~m}$ & $\mathrm{CT}$ & $\mathrm{IC}_{50} 28.7 \mu \mathrm{M}($ ECA-109) & [267] \\
\hline $\begin{array}{l}415 \\
416 \\
417 \\
418 \\
419\end{array}$ & Aspergillus wentii SD-310 & Deep-sea $2038 \mathrm{~m}$ & $\begin{array}{l}\mathrm{AB} \\
\mathrm{AF}\end{array}$ & $\begin{array}{l}\text { MIC 8, 32, 8, } 8 \mu \mathrm{g} / \mathrm{mL} \text { (E. tarda, P. aeruginosa, } \\
\text { V. harveyi, V. parahaemolyticus) } \\
\text { MIC 32, 8, 8, } 8 \mu \mathrm{g} / \mathrm{mL}(\text { E. coli, E. tarda, } \\
\text { V. harveyi, V. parahaemolyticus) } \\
\text { MIC } 32 \mu \mathrm{g} / \mathrm{mL} \text { (Aeromonas hydrophilia, } \\
\text { V. anguillarum) } \\
\text { MIC } 32,32 \mu \mathrm{g} / \mathrm{mL} \text { (E. tarda, V. harveyi) } \\
\text { MIC } 32,32 \mu \mathrm{g} / \mathrm{mL} \text { (V. anguillarum, V. harveyi) } \\
\text { MIC } 4 \mu \mathrm{g} / \mathrm{mL}(\text { F. graminearum })\end{array}$ & [278] \\
\hline $\begin{array}{r}432 \\
438 \\
457 \\
456 \\
455\end{array}$ & Sarocladium kiliense & Deep-sea $5070 \mathrm{~m}$ & CT & $\begin{array}{l}\mathbf{I C}_{50} 25.8 \boldsymbol{\mu M}(\mathrm{HeLa}-\mathrm{S} 3) \\
\mathbf{I C}_{50} 9.2 \mu \mathrm{M}(\mathrm{HeLa}-\mathrm{S} 3) \\
\mathbf{I C}_{50} \mathbf{4 4 . 2} \boldsymbol{M M}(\mathrm{HeLa}-\mathrm{S} 3) \\
\mathbf{I C}_{50} 9.3 \mu \mathrm{M}(\mathrm{HeLa}-\mathrm{S} 3) \\
\mathbf{I C}_{50} 30.1 \boldsymbol{M M}(\mathrm{HeLa}-\mathrm{S} 3) \\
\mathbf{I C}_{50} 70 \boldsymbol{M}(\mathrm{A} 549)[293]\end{array}$ & [290] \\
\hline
\end{tabular}

[170]. Compounds that contain the $\mathrm{N}-\mathrm{N}$ bond are quite rare among natural compounds [323] but they are widely represented among synthetic biologically active compounds, especially pyridazine derivatives [324].

New cytotoxic meroterpenoids, eutypellacytosporins A-D (507-510), were isolated from Arctic fungus Eutypella sp. D11 [325]. These compounds are conjugates of polyketide (cytosporin D) and sesquiterpenoids (decipienolides $\mathrm{A}$ and $\mathrm{B}$ ). A small difference in the activity of the C-21 epimers (507) and (509) indicates a slight influence of the configuration of this optical center.

The meroterpenoid austinol (511) [326], which was isolated among secondary metabolites from a thermophilic fungus of the Penicillium genus [73], showed a noticeable antibacterial activity.
The chemical study of the extract of deep-sea fungus Penicillium sp. YPGA11 [327] led to the isolation of four new antibacterial meroterpenoids farnesylcyclohexenones, peniginsengins $\mathrm{B}-\mathrm{E}(\mathbf{5 0 3}-\mathbf{5 0 6})$, that contained rare motifs for natural compounds, i.e., 1-methylcyclohexene cycle and $(4 E, 8 E)$-4,8-dimethyldeca-4,8-dienoic acid.

\section{CONCLUSIONS}

The search for new antibiotics is an increasingly urgent task in the context of the spread of drug resistance. Different strategies were used in different periods to search for natural biologically active compounds. The most productive was the Waksman platform, which consists in the cultivation of soil microorganisms with a wide range of antibiotic activ- 
Table 7. Data on the origin and biological activity of compounds of mixed and unidentified origin

\begin{tabular}{|c|c|c|c|c|c|}
\hline Cpd. & Producer/species & $\begin{array}{l}\text { Extremophile } \\
\text { type/origin }\end{array}$ & $\begin{array}{l}\text { Activity/ } \\
\text { toxicity }\end{array}$ & $\mathrm{EC}_{50}, \mathrm{IC}_{50}, \mathrm{MIC}$ & Ref. \\
\hline 458 & $\begin{array}{l}\text { Penicillium sp. } \\
\text { ZZ1283 }\end{array}$ & Marine & $\begin{array}{l}\mathrm{AB} \\
\mathrm{AF}\end{array}$ & $\begin{array}{l}\text { MIC 4, } 3 \mu \mathrm{g} / \mathrm{mL}(S . \text { aureus, E. coli }) \\
\text { MIC } 8 \mu \mathrm{g} / \mathrm{mL}(C . \text { albicans })\end{array}$ & [301] \\
\hline 460 & $\begin{array}{l}\text { Aspergillus ochra- } \\
\text { ceus }\end{array}$ & Marine & CT & $\begin{array}{l}\text { IC }_{\mathbf{5 0}} \mathbf{0 . 8}-\mathbf{1 . 0} \boldsymbol{\mu g} / \mathbf{m L}(\mathrm{BME}-\mathrm{UV} 1, \mathrm{MDCK}) \\
{[328]} \\
\mathbf{I C}_{\mathbf{5 0}} \mathbf{1 7 - 8 7} \boldsymbol{\mu M}(\mathrm{IHKE})[329] \\
\text { IC }_{\mathbf{5 0}} \mathbf{0 . 3} \boldsymbol{\mu M}(\mathrm{Hep} \mathrm{G} 2)[330] \\
\text { IC }_{\mathbf{5 0}} \mathbf{3} \boldsymbol{\mu M}(\mathrm{A} 2780)\end{array}$ & [42] \\
\hline 461 & $\begin{array}{l}\text { Aspergillus sp. } \\
\text { SCSIO41211 }\end{array}$ & Mangrove & CT & $\begin{array}{l}\text { IC }_{\mathbf{5 0}} \mathbf{0 . 0 3 1 - 2 3 . 2} \boldsymbol{\mu M} \text { (H1975, U937, K562, BGC823, } \\
\text { MOLT-4, MCF-7, A549, Hela, HL60, Huh-7) }\end{array}$ & [254] \\
\hline $\begin{array}{r}463 \\
468 \\
469 \\
470\end{array}$ & $\begin{array}{l}\text { Trichoderma eri- } \\
\text { naceum } \\
\text { F1-1 }\end{array}$ & Marine & CT & $\begin{array}{l}\mathrm{IC}_{50} 31.9 \mu \mathrm{M}(\mathrm{MDA}-\mathrm{MB}-435) \\
\mathrm{IC}_{\mathbf{5 0}} 12.5 \mu \mathrm{M}(\mathrm{MDA}-\mathrm{MB}-435) \\
\mathrm{IC}_{50} 41.4 \mu \mathrm{M}(\mathrm{MDA}-\mathrm{MB}-435) \\
\mathrm{IC}_{\mathbf{5 0}} 22.3, \mathbf{1 8 . 4} \boldsymbol{\mu M}(\mathrm{MDA}-\mathrm{MB}-435, \mathrm{~A} 549)\end{array}$ & [270] \\
\hline $\begin{array}{l}471 \\
472\end{array}$ & $\begin{array}{l}\text { Truncatella angus- } \\
\text { tata }\end{array}$ & Marine & AV & $\begin{array}{l}\mathrm{IC}_{50} 39 \boldsymbol{\mu M}(\mathrm{HIV}-1) \\
\mathrm{IC}_{50} 16.1 \mu \mathrm{M}(\mathrm{HIV}-1)\end{array}$ & [307] \\
\hline 473 & $\begin{array}{l}\text { Penicillium sp. } \\
\text { LS54. }\end{array}$ & Marine & $\mathrm{AB}$ & MIC $8 \mu \mathrm{g} / \mathrm{mL}$ ( $V$. harveyi) & [308] \\
\hline $\begin{array}{l}476 \\
477\end{array}$ & $\begin{array}{l}\text { Aspergillus versi- } \\
\text { color } \\
\text { DJ013 }\end{array}$ & Marine & $\mathrm{AF}$ & $\begin{array}{l}\text { MIC } 64 \mu \mathrm{g} / \mathrm{mL} \text { ( G. graminis, C. neoformans, C. albicans) } \\
\text { MIC } 64 \mu \mathrm{g} / \mathrm{mL} \text { (G. graminis, C. neoformans, C. albicans) }\end{array}$ & [310] \\
\hline 478 & $\begin{array}{l}\text { Cladosporium sp. } \\
\text { JS } 1-2\end{array}$ & Mangrove & $\mathrm{AB}$ & MIC $25,25,12.5 \mu \mathrm{g} / \mathrm{mL}$ (S. aureus, E. coli, B. cereus $)$ & [121] \\
\hline $\begin{array}{l}479 \\
480\end{array}$ & $\begin{array}{l}\text { Talaromyces } \\
\text { assiutensis } \\
\text { JTY2 }\end{array}$ & Mangrove & $\mathrm{AB}$ & $\begin{array}{l}\text { MIC 20, } 40 \mu \mathrm{g} / \mathrm{mL}(S . \text { aureus, E. coli }) \\
\text { MIC 5, 2.5, 5, 2.5, 2.5, } 1.25 \mu \mathrm{g} / \mathrm{mL}(M . \text { tetragenus, } S . \\
\text { aureus, } S . \text { albus, B. cereus, B.subtilis, E. coli) }\end{array}$ & [311] \\
\hline 482 & $\begin{array}{l}\text { Penicillium sp. } \\
\text { TGM112 }\end{array}$ & Mangrove & $\mathrm{AB}$ & MIC $6.25 \mu \mathrm{g} / \mathrm{mL}(S$. aureus $)$ & [312] \\
\hline 488 & $\begin{array}{l}\text { Penicillium her- } \\
\text { quei JX4 }\end{array}$ & Mangrove & $\mathrm{AF}$ & 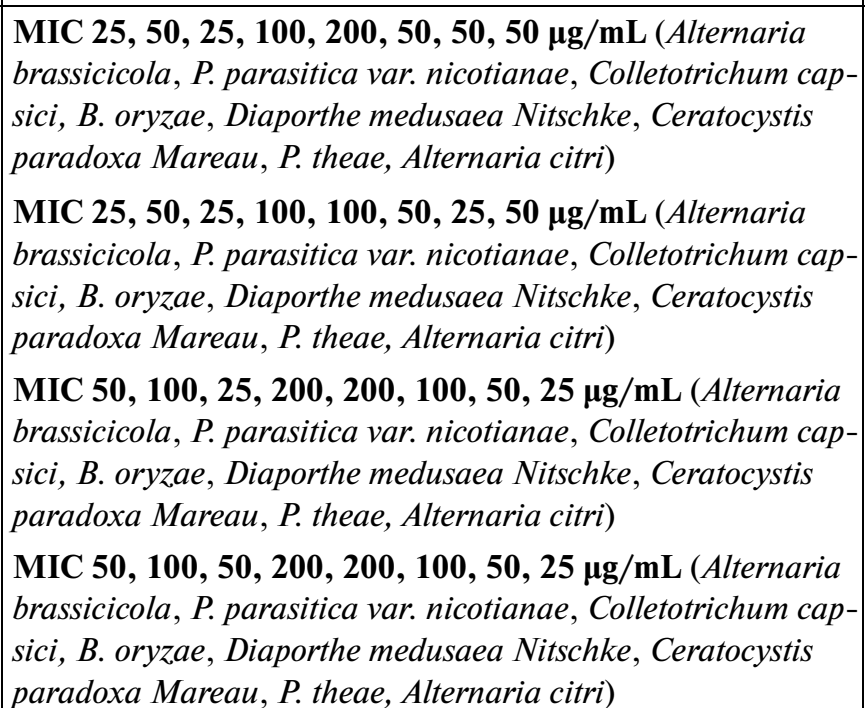 & [313] \\
\hline
\end{tabular}


Table 7. (Contd.)

\begin{tabular}{|c|c|c|c|c|c|}
\hline Cpd. & Producer/species & $\begin{array}{l}\text { Extremophile } \\
\text { type/origin }\end{array}$ & $\begin{array}{l}\text { Activity/ } \\
\text { toxicity }\end{array}$ & $\mathrm{EC}_{50}, \mathrm{IC}_{50}, \mathrm{MIC}$ & Ref. \\
\hline 497 & $\begin{array}{l}\text { Cladosporium sp. } \\
\text { JJM22 }\end{array}$ & Mangrove & $\mathrm{AB}$ & $\begin{array}{l}\text { IC }_{50} 20 \mu \mathrm{M} \text { (S. aureus, B. cereus, MRSA, E. coli, V. algino- } \\
\text { lyticus, V. parahemolyticus) } \\
\text { MIC } 200 \mu \mathrm{g} / \mathbf{m L}(S . \text { aureus, MRSA, M. gypseum SH-MU- } \\
\text { 4) [315] }\end{array}$ & [314] \\
\hline 490 & $\begin{array}{l}\text { Aspergillus terreus } \\
\text { LGO13 }\end{array}$ & Marine & $\begin{array}{l}\mathrm{AB} \\
\mathrm{AF} \\
\mathrm{CT}\end{array}$ & 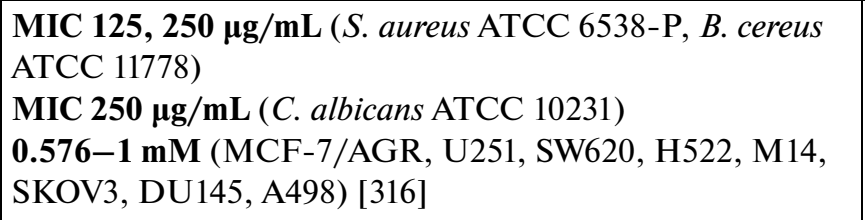 & \\
\hline 491 & & & & $\begin{array}{l}\text { Qualitative AF activity (C. albicans ATCC 10231) } \\
\text { 0.626-1 mM (MCF-7/AGR, U251, SW620, H522, M14, } \\
\text { SKOV3, DU145, A498) [316] }\end{array}$ & [287] \\
\hline 492 & & & & $\begin{array}{l}\text { MIC } 250.0 \mu \mathrm{g} / \mathbf{m L} \text { (B. cereus ATCC 11778) } \\
\mathbf{0 . 1 6 - 1 ~ m M ~ ( M C F - 7 / A G R , ~ U 2 5 1 , ~ S W 6 2 0 , ~ H 5 2 2 , ~ M 1 4 , ~} \\
\text { SKOV3, DU145, A498) [316] }\end{array}$ & \\
\hline 498 & & & & $\begin{array}{l}\text { MIC 62.5, 250, } 250 \mu \mathrm{g} / \mathbf{m L}(S . \text { aureus ATCC 6538-P, } S \text {. } \\
\text { cerevisiae ATCC } 9080, \text { B. cereus ATCC 11778) } \\
\text { MIC } 250 \mu \mathrm{g} / \mathbf{m L}(\text { C. albicans ATCC 10231) }\end{array}$ & \\
\hline 493 & $\begin{array}{l}\text { Aspergillus terreus } \\
\text { SCSIO FZQ028 }\end{array}$ & $\begin{array}{l}\text { Deep-sea } \\
1718 \mathrm{~m}\end{array}$ & $\mathrm{AB}$ & $\begin{array}{l}\text { Qualitative AB activity (S. aureus, B. thuringiensis, B. subti- } \\
\text { lis, E. coli) } \\
\text { Qualitative AB activity (S. aureus, B. thuringiensis, B. subti- } \\
\text { lis, E. coli) }\end{array}$ & [318] \\
\hline $\begin{array}{l}500 \\
501 \\
502\end{array}$ & Aspergillus niger & Marine & $\mathrm{AB}$ & $\begin{array}{l}\text { MIC } 16 \mu \mathrm{g} / \mathrm{mL}(S . \text { aureus }) \\
\text { MIC } 32 \mu \mathrm{g} / \mathrm{mL}(S . \text { aureus }) \\
\text { MIC } 32 \mu \mathrm{g} / \mathrm{mL}(S \text {. aureus })\end{array}$ & {$[322]$} \\
\hline 513 & $\begin{array}{l}\text { Aspergillus versi- } \\
\text { color } \mathrm{MCCC} \\
\text { 3A00080 }\end{array}$ & $\begin{array}{l}\text { Deep-sea } \\
2721 \mathrm{~m}\end{array}$ & $\mathrm{AB}$ & MIC $8 \mu \mathrm{g} / \mathrm{mL}$ (S. aureus) & {$[321]$} \\
\hline $\begin{array}{l}474 \\
475\end{array}$ & $\begin{array}{l}\text { Phyllosticta capi- } \\
\text { talensis }\end{array}$ & Mangrove & $\mathrm{AB}$ & 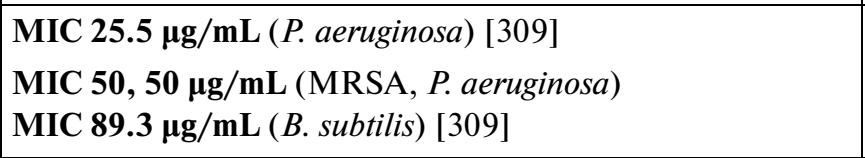 & [141] \\
\hline $\begin{array}{l}507 \\
508 \\
509 \\
510\end{array}$ & Eutypella sp. D-1 & Arctic & $\mathrm{CT}$ & $\begin{array}{l}\text { IC }_{\mathbf{5 0}} \text { 7.3-17.1 } \boldsymbol{\mu M} \text { (DU145, SW1990, Huh7, PANC-1) } \\
\text { IC }_{\mathbf{5 0}} \text { 4.9-11.0 } \boldsymbol{\mu M} \text { (DU145, SW1990, Huh7, PANC-1) } \\
\text { IC }_{\mathbf{5 0}} \text { 9.6-13.5 } \boldsymbol{\mu M} \text { (DU145, SW1990, Huh7, PANC-1) } \\
\text { IC }_{\mathbf{5 0}} \text { 7.5-13.4 } \boldsymbol{\mu M} \text { (DU145, SW1990, Huh7, PANC-1) }\end{array}$ & {$[325]$} \\
\hline 511 & $\begin{array}{l}\text { Penicillium sp. } \\
\text { RO-11 }\end{array}$ & $\begin{array}{l}\text { Hot spring } \\
\text { deposits } \\
\left(45-65^{\circ} \mathrm{C}\right) \\
\text { Saudi Arabia/ } \\
\text { Thermophile }\end{array}$ & $\begin{array}{l}\mathrm{AB} \\
\mathrm{CT}\end{array}$ & $\begin{array}{l}\text { MIC 1.4, 2.5, 0.13 } \mu \mathrm{g} / \mathrm{mL}(S . \text { aureus, Escherichia fergu- } \\
\text { sonii, } P . \text { aeruginosa) } \\
\text { IC }_{\mathbf{5 0}} 10 \mu \mathrm{M}(\mathrm{HTB}-176)\end{array}$ & [73] \\
\hline $\begin{array}{l}504 \\
505 \\
506\end{array}$ & $\begin{array}{l}\text { Penicillium sp. } \\
\text { YPGA11 }\end{array}$ & $\begin{array}{l}\text { Deep-sea } \\
4500 \mathrm{~m}\end{array}$ & $\mathrm{AB}$ & $\begin{array}{l}\text { MIC } 32,8 \boldsymbol{\mu g} / \mathbf{m L}(S . \text { aureus ATCC } 43300, S . \text { aureus ATCC } \\
25913) \\
\text { MIC } 16 \boldsymbol{\mu g} / \mathbf{m L}(S . \text { aureus ATCC } 25913) \\
\text { MIC } 64,32 \boldsymbol{\mu g} / \mathbf{m L}(S . \text { aureus ATCC } 43300, S \text {. aureus } \\
\text { ATCC } 25913)\end{array}$ & [327] \\
\hline
\end{tabular}




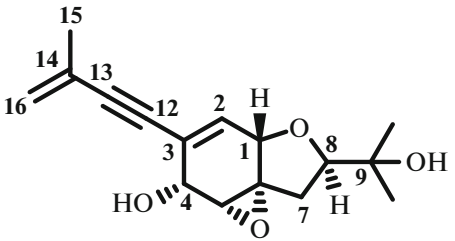

Truncateols $\mathrm{O}, \mathrm{P}$

(471) $4(R)$

(472) $4(S)$

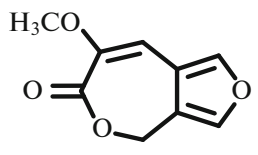

Penicillilactone A

(473)

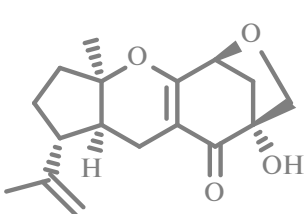

Guignardone A

(474)

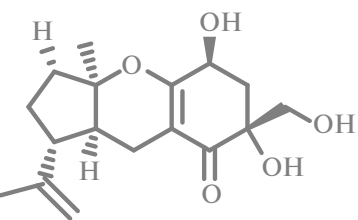

Guignardone J

(475)

Fig. 30. Trunkateols, penicillacton A, and guignardones.<smiles>[R]CC([R])CCCCC1=C(C)C(=O)OC1=O</smiles><smiles>CCCC(=O)[C@@H]1C=C(C)C(=O)[C@@H]1C(=O)O[C@H](C)CC(=O)O</smiles>

Asperfurandiones A, B

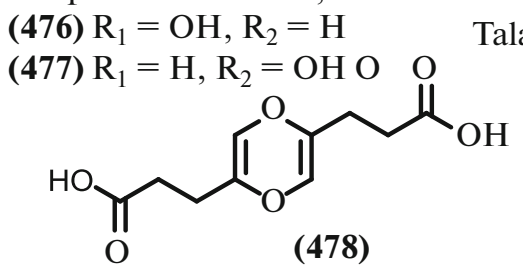<smiles>[R20]CCCCCCC[C@H](C(=C)C(=O)O)C(=O)OC</smiles>

Talarocyclopenta A

(479)

Talarocyclopenta B

(480)
Talarocyclopenta C

(481)

Fig. 31. Asperfurandiones and talarocyclopentas.<smiles>CCCC1=CC(=C[C@H](O)[C@@H](C)O)OC1=O</smiles>

Penicilactone A (482) 6(S), 7(S)

Penicilactone B (483) 6(S), 7(R)

Penicilactone $\mathrm{C}$ (484) $6(R), 7(S)$<smiles>C/C=C\C(=C/C=C/C(c1ccc(O)c(OC)c1)(C(C)O)C(C)O)CO</smiles>

Penicilquei A (485) 3(R)

Penicilquei B

(486) 3(S)<smiles>C/C=C\C(=C/[C@H](/C=C(/C)CO)c1cc(NC(C)=O)c(OC)cc1O)CO</smiles>

Penicilquei $\mathrm{C}$ (487) 5(R)

Penicilquei D (488) $5(S)$

Fig. 32. Penicilactons, penicilqueis, and alternaton A.

ity [331]. This approach allowed to identify of the majority of currently known antibiotics including those used in medicine and veterinary medicine. However, the chances of revealing new compounds by this method after the 1970s became negligible because of the problem of rediscovering known antibiotics (rediscovery problem). At the same time, the need for new antibiotic agents is only growing because of the spread of resistance, which has led to the emergence of alternative approaches, such as omics methods (metabolomics, genomics, and reverse genomics) and target-oriented high-performance screening [1]. 


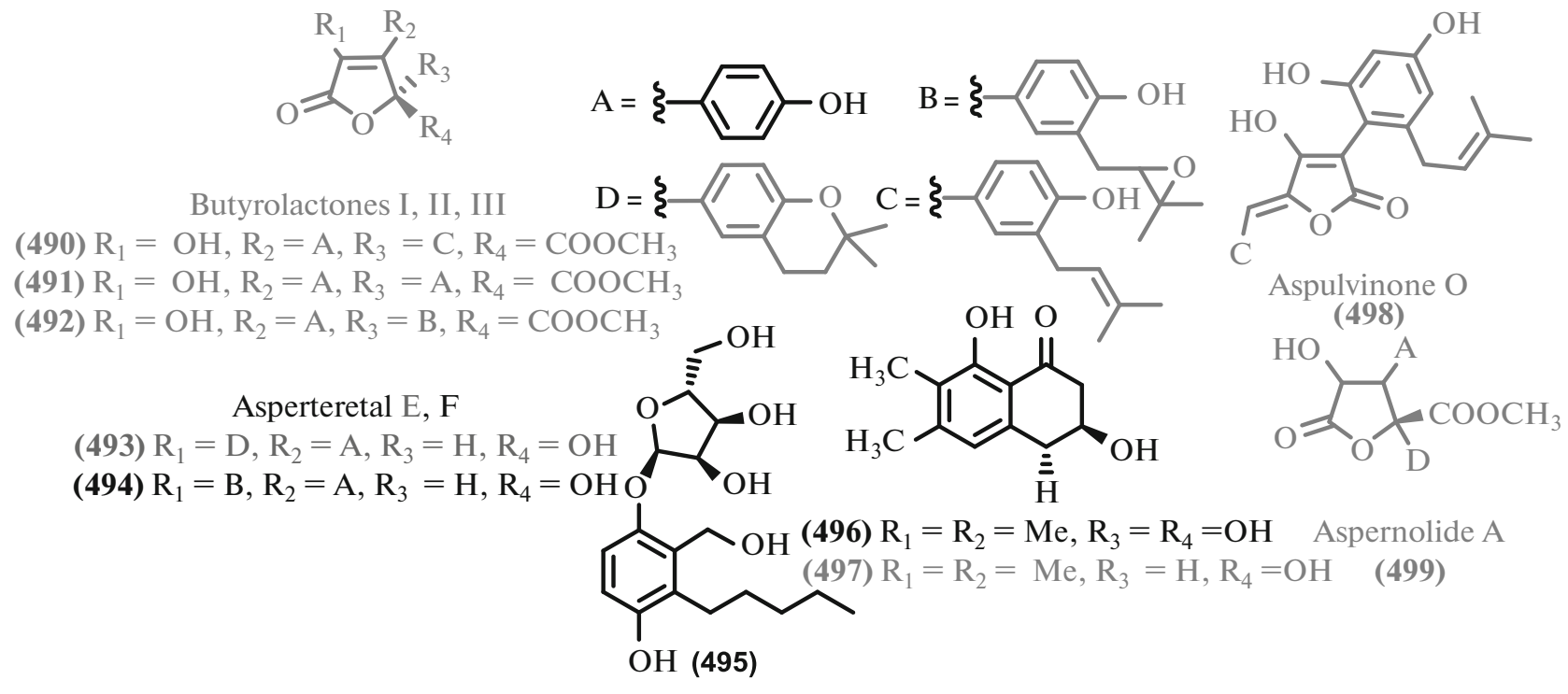

Fig. 33. Butyrolactones and phenols.<smiles>[R]CCCC[C@H](C(=C)C(=O)O)C(=O)O[R9]</smiles>

Asperitaconic acids $\mathrm{A}-\mathrm{C}$ (500) $\mathrm{R}_{1}=\mathrm{CH}_{3}, \mathrm{R}_{2}=\mathrm{CH}_{2} \mathrm{CH}_{2} \mathrm{OH}$ (501) $\mathrm{R}_{1}=\mathrm{H}, \mathrm{R}_{2}=\mathrm{CH}_{2} \mathrm{CH}_{2} \mathrm{OAc}$ (502) $\mathrm{R}_{1}=\mathrm{H}, \mathrm{R}_{2}=\mathrm{COCH}_{3}$<smiles>[R][C@]1([2H])CC(=O)C(C)=CC1=O</smiles><smiles>[R][C@]1([2H])C(=O)C=C(C)[C@@H](O)[C@H]1O</smiles>

Peniginsengine B (503)<smiles>[R]#CC/C=C(/C)CC/C=C(\C)CCC(=O)O</smiles><smiles>[R]C1(O)C[C@H](O)C(C)=C[C@@H]1O</smiles>

Peniginsengine D (505)

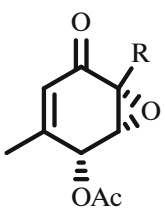

Peniginsengine E (506)<smiles>COc1ccc(O)cc1-c1cc(O)ccc1OC</smiles>

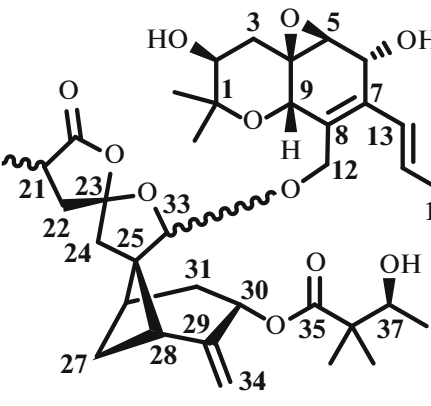

15

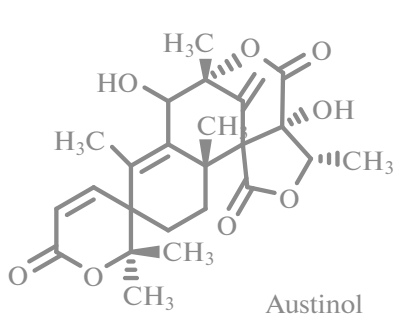

(511)
(512)

(508) $33(S), 21(R)$

(509) $33(R), 21(R)$

(510) $33(R), 21(S)$

Fig. 34. Meroterpenoids and other metabolites of mixed origin.

However, none of the developed strategies has led to a breakthrough in the search for antibiotics.

One of the ways to overcome the crisis in the search for natural compounds is to study difficult-to-cultivate and rare microorganisms including the inhabitants of extreme ecosystems [332, 333]. To assess the prospects for this approach, we have analyzed all secondary metabolites of extreme micromycetes, which are new or known compounds with antibiotic activity isolated over the period 2018-2019.

From a taxonomic point of view, most micromycetes that have become sources of new and/or known active compounds belong to the common Aspergillus and Penicillium genera. Representatives of other taxa have much smaller proportions. 


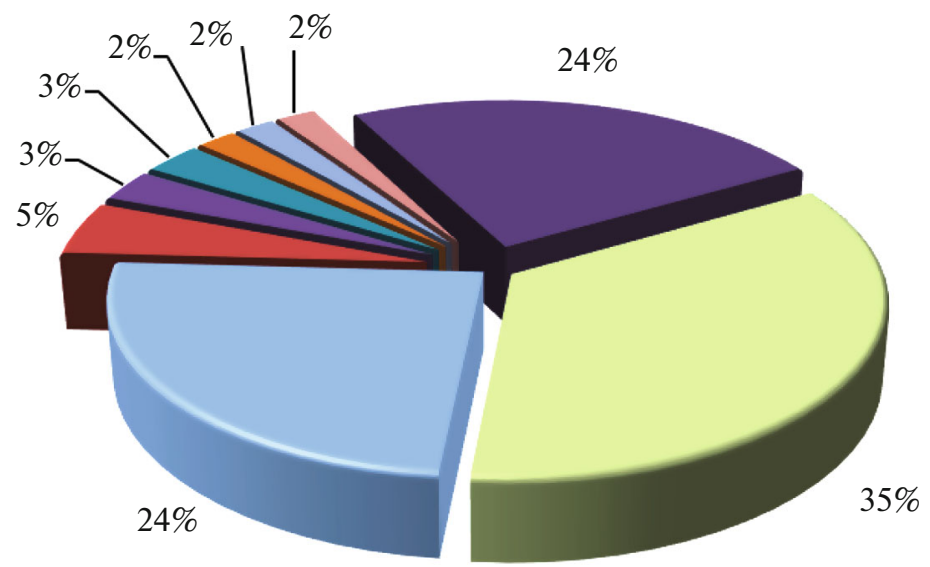

$\begin{array}{lll}\text { Aspergillus } & \text { Menicillium } & \text { Cladosporium } \\ \text { Gusarium } & \text { M Alternaria } & \text { Trichoderma } \\ \text { Talaromyces } & \text { M Curvularia } & \text { Others }\end{array}$

Fig. 35. Distribution of extremophilic micromycetes by genera.

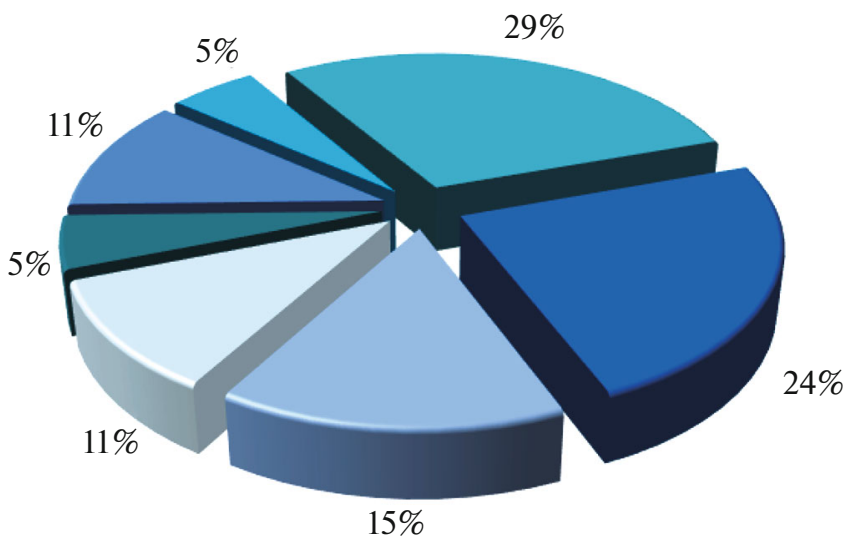
国 Peptides
国 Chromones and xantones $\mathbf{a}$ Quinones
回Other polyketides
回 Terpenes
Alkaloids
Mixed

Fig. 36. Structural families of metabolites isolated from extremophilic micromycetes.

From a biogenetic point of view, most of the metabolites were polyketides $(45 \%)$ of various structures. Peptides and related compounds were very rare for extremophilic micromycetes $(5 \%)$. The distribution of metabolites by structural families is shown in Fig. 36.

Many metabolites (about one third (37\%) of all compounds, 190 out of 513), the structure of which was established in the covered period, were previously described antibiotics, and the spectrum of biological activity was clarified for some of them. The antibiotic activity was shown in 53\% (179 compounds) of the previously undescribed metabolites (323 compounds, $63 \%$ of the total). At the same time, about half of all active metabolites (369) were new. It can be assumed that these statistics do not cover all known antibiotics isolated during the period studied because the data often do not reach publication in the case of isolation of the known compounds.

Despite the short coverage period (2018-2019), many new natural compounds mentioned in the review became the object of the full synthesis. In par- 


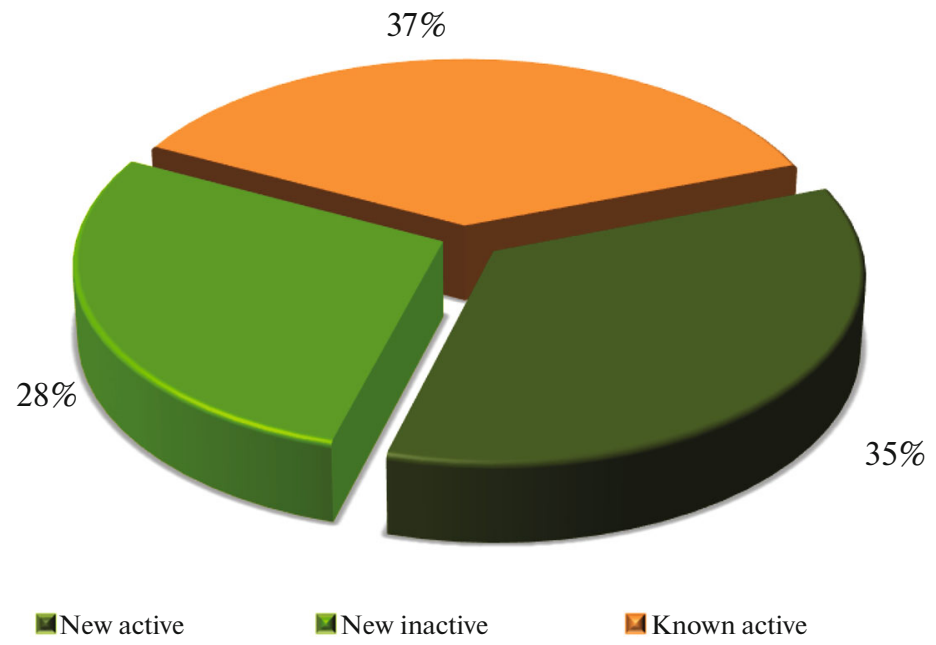

Fig. 37. Distribution of activity of new and previously described metabolites of extremophilic micromycetes.

ticular, peptaibols lipovelutibols B (24) and D (26) and their lipophilic analogs were obtained by the solid-phase method [334]. Terpenoids insulicolide (354) and 14- $O$-acetylinsulicolide (355) and their analogs were synthesized by the enantioselective metal-catalyzed assembly of the driman core followed by oxidative degradation and functionalization [335]. We should also mention the counter synthesis of the indole alkaloid misszrtina A (294) [241].

The generalized data show that many new secondary metabolites were isolated from extremophilic micromycetes, although known antibiotic compounds make up a high proportion. In other words, only studying extreme and hard-to-reach habitats does not solve the rediscovery problem of known antibiotics. Many of the known compounds isolated according to the bioactivity screening have been obtained from different producers many times. For example, antibiotic anthraquinone emodin (35) was developed and characterized seven times during the period considered [61, 73-79]. The use of the modern omics approaches and prioritization and dereplication strategies [336] can significantly increase the efficiency of the study of natural compounds including those from extremophilic econiches, thus avoiding the resource overuse for the isolation of known compounds. In this case, extremophilic microorganisms may have a high potential as sources of new drugs because of the high diversity of chemical structures and biological activities of secondary metabolites of these microorganisms.

\section{FUNDING}

The work was supported by the Russian Foundation of Basic Research (grant no. 19-14-50448).

\section{COMPLIANCE WITH ETHICAL STANDARDS}

This article does not contain any studies involving animals or humans as research subjects.

\section{Conflict of Interests}

The authors state that they have no conflict of interest.

\section{ADDITIONAL INFORMATION}

Review article of the winners of the Russian Foundation of Basic Research competition "Expansion”, 2019.

\section{REFERENCES}

1. Ribeiro da Cunha, B., Fonseca, L.P., and Calado, C.R.C., Antibiotics, 2019, vol. 8, p. 45. https://doi.org/10.3390/antibiotics8020045

2. Dougan, G., Dowson, C., and Overington, J., Drug Discov. Today, 2019, vol. 24, pp. 452-461. https://doi.org/10.1016/j.drudis.2018.11.015

3. Political Declaration of the High-Level Meeting of the General Assembly on Antimicrobial Resistance, United Nations Resolution A/RES/71/3 (adopted October 5, 2016). https://undocs.org/ru/A/RES/71/3.

4. Global Action Plan to Combat Antimicrobial Resistance, Geneva: World Health Organization, 2015, pp. 15-29.

5. Strategy for Preventing the Spread of Antimicrobial Resistance in the Russian Federation for the Period up to 2030, Order of the Government of the Russian Federation dated September 25, 2017, no. 2045-r, pp. 2-14.

6. Newman, D.J. and Cragg, G.M., J. Nat. Prod., 2020, vol. 83, pp. $770-803$.

https://doi.org/10.1021/acs.jnatprod.9b01285

7. Sayed, A.M., Hassan, M.H.A., Alhadrami, H.A., Hassan, H.M., Goodfellow, M., and Rateb, M.E., $J$. Appl. Microbiol., 2020, vol. 128, pp. 630-657. https://doi.org/10.1111/jam.14386 
8. Keller, N.P., Nat. Rev. Microbiol., 2019, vol. 17, pp. $167-180$. https://doi.org/10.1038/s41579-018-0121-1

9. Schueffler, A. and Anke, T., Nat. Prod. Rep., vol. 31, pp. $1425-1448$. https://doi.org/10.1039/C4NP00060A

10. Zhang, X., Li, S.-J., Li, J.-J., Liang, Z.-Z., and Zhao, C.-Q., Mar. Drugs, 2018, vol. 16, p. 194. https://doi.org/10.3390/md16060194

11. Wickneswary, N., Abu, BakarM., and Khalid, R., Malaysian J. Chem., 2016, vol. 18, pp. 100-105.

12. Tian, Y., Li, Y.-L., and Zhao, F.-C., Mar. Drugs, 2017, vol. 15 , p. 28.

https://doi.org/10.3390/md15030028

13. Stierle, A.A. and Stierle, D.B., Nat. Prod. Commun., 2014, vol. 9, pp. 1037-1044.

https://doi.org/10.1177/1934578X1400900738

14. Corral, P., Amoozegar, M.A., and Ventosa, A., Mar. Drugs, 2019, vol. 18, p. 33. https://doi.org/10.3390/md18010033

15. Tiquia-Arashiro, S.M. and Grube, M., Fungi in Extreme Environments: Ecological Role and Biotechnological Significance, Springer Int. Publ., 2019. https://doi.org/10.1007/978-3-030-19030-9

16. Giddings, L.-A. and Newman, D.J., Bioactive compounds from extremophilic marine fungi, in Fungi in Extreme Environments: Ecological Role and Biotechnological Significance, Tiquia-Arashiro, S.M. and Grube, M., Eds., Springer Int. Publ., 2019, pp. 349-382. https://doi.org/10.1007/978-3-030-19030-9_18

17. Zhang, X., Li, S.-J., Li, J.-J., Liang, Z.-Z., and Zhao, C.-Q., Mar. Drugs, 2018, vol. 16, p. 194. https://doi.org/10.3390/md16060194

18. Gribble, G., Mar. Drugs, 2015, vol. 13, pp. 4044-4136. https://doi.org/10.3390/md13074044

19. Xu, L., Meng, W., Cao, C., Wang, J., Shan, W., and Wang, Q., Mar. Drugs, 2015, vol. 13, pp. 3479-3513. https://doi.org/10.3390/md13063479

20. Jin, L., Quan, C., Hou, X., and Fan, S., Mar. Drugs, 2016, vol. 14, p. 76. https://doi.org/10.3390/md14040076

21. Ma, H.-G., Liu, Q., Zhu, G.-L., Liu, H.-S., and Zhu, W.-M., J. Asian Nat. Prod. Res., 2016, vol. 18, pp. $92-115$.

https://doi.org/10.1080/10286020.2015.1127230

22. Fouillaud, M., Venkatachalam, M., Girard-Valenciennes, E., Caro, Y., and Dufosse, L., Mar. Drugs, 2016, vol. 14,64 .

https://doi.org/10.3390/md14040064

23. Imhoff, J., Mar. Drugs, 2016, vol. 14, p. 19. https://doi.org/10.3390/md14010019

24. El-Hossary, E.M., Cheng, C., Hamed, M.M., ElSayed, HamedA.N., Ohlsen, K., Hentschel, U., and Abdelmohsen, U.R., Eur. J. Med. Chem., 2017, vol. 126 , pp. $631-651$.

https://doi.org/10.1016/j.ejmech.2016.11.022

25. Nicoletti, R., Salvatore, M., and Andolfi, A., Mar. Drugs, 2018, vol. 16, p. 12. https://doi.org/10.3390/md16010012

26. Tortorella, E., Tedesco, P., Palma Esposito, F., Fanuary, G., Fani, R., Jaspars, M., and de Pascale, D.,
Mar. Drugs, 2018, vol. 16, p. 355. https://doi.org/10.3390/md16100355

27. Ancheeva, E., Daletos, G., and Proksch, P., Mar. Drugs, 2018, vol. 16, p. 319. https://doi.org/10.3390/md16090319

28. Rai, M., Gade, A., Zimowska, B., Ingle, A.P., and Ingle, P., Appl. Microbiol. Biotechnol., 2018, vol. 102, pp. 9053-9066. https://doi.org/10.1007/s00253-018-9329-2

29. Deshmukh, S.K., Prakash, V., and Ranjan, N., Front. Microbiol., 2018, vol. 8, p. 2536. https://doi.org/10.3389/fmicb.2017.02536

30. Wang, K.-W. and Ding, P., Mini-Rev. Med. Chem., 2018, vol. 18, pp. 1072-1094. https://doi.org/10.2174/1389557518666180305160856

31. Barone, G., Varrella, S., Tangherlini, M., Rastelli, E., Dell'Anno, A., Danovaro, R., and Corinaldesi, C., Diversity, 2019, vol. 11, p. 113. https://doi.org/10.3390/d11070113

32. Youssef, F.S., Ashour, M.L., Singab, A.N.B., and Wink, M., Mar. Drugs, 2019, vol. 17, p. 559. https://doi.org/10.3390/md17100559

33. Carroll, A.R., Copp, B.R., Davis, R.A., Keyzers, R.A., and Prinsep, M.R., Nat. Prod. Rep., 2019, vol. 36, pp. $122-173$. https://doi.org/10.1039/c8np00092a

34. Sun, W., Wu, W., Liu, X., Zaleta-Pinet, D.A., and Clark, B.R., Mar. Drugs, 2019, vol. 17, p. 339. https://doi.org/10.3390/md17060339

35. Arifeen, M., Ma, Y.-N., Xue, Y.-R., and Liu, C.-H., Mar. Drugs, 2019, vol. 18, p. 9. https://doi.org/10.3390/md18010009

36. Luo, M., Zang, R., Wang, X., Chen, Z., Song, X., Ju, J., and Huang, H., J. Nat. Prod., 2019, vol. 82, pp. 25942600. https://doi.org/10.1021/acs.jnatprod.9b00545

37. Youssef, D. and Alahdal, A., Molecules, 2018, vol. 23, p. 394 https://doi.org/10.3390/molecules23020394

38. Adamczeski, M., Reed, A.R., and Crews, P., J. Nat. Prod., 1995, vol. 58, pp. 201-208. https://doi.org/10.1021/np50116a007

39. Van, G., Dai, Sh., Chen, M., Vu, Kh., Shi, L., Lyuo, S., and Li, S., Khim. Prirodn. Soed., 2010, vol. 46, no. 4, pp. 491-493.

40. Liu, C.-C., Zhang, Z.-Z., Feng, Y.-Y., Gu, Q.-Q., Li, D.-H., and Zhu, T.-J., Nat. Prod. Res., 2019, vol. 33, pp. 414-419. https://doi.org/10.1080/14786419.2018.1455045

41. Guimarães, D.O., Borges, W.S., Vieira, N.J., de Oliveira, L.F., da Silva, C.H.T.P., Lopes, N.P., Dias, L.G., Durán-Patrón, R., Collado, I.G., and Pupo, M.T., Phytochemistry, 2010, vol. 71, pp. 1423-1429. https://doi.org/10.1016/j.phytochem.2010.05.012

42. Frank, M., Özkaya, F., Müller, W., Hamacher, A., Kassack, M., Lin, W., Liu, Z., and Proksch, P., Mar. Drugs, 2019, vol. 17, p. 99. https://doi.org/10.3390/md17020099

43. Kimura, Y., Tani, K., Kojima, A., Sotoma, G., Okada, K., and Shimada, A., Phytochemistry, 1996, vol. 41, 
pp. $665-669$.

https://doi.org/10.1016/0031-9422(95)00693-1

44. Li, W., Luo, D., Huang, J., Wang, L., Zhang, F., Xi, T., Liao, J., and Lu, Y., Nat. Prod. Res., 2018, vol. 32, pp. 662-667.

https://doi.org/10.1080/14786419.2017.1335730

45. Hodge, R.P., Harris, C.M., and Harris, T.M., J. Nat. Prod., 1988, vol. 51, pp. 66-73. https://doi.org/10.1021/np50055a008

46. Niu, S., Xia, M., Chen, M., Liu, X., Li, Z., Xie, Y., Shao, Z., and Zhang, G., Mar. Drugs, 2019, vol. 17, p. 686. https://doi.org/10.3390/md17120686

47. Niu, S., Wang, N., Xie, C.-L., Fan, Z., Luo, Z., Chen, H.-F., and Yang, X.-W., J. Antibiot., 2018, vol. 71, pp. 658-661. https://doi.org/10.1038/s41429-018-0046-y

48. Kopp, B. and Rehm, H.J., Eur. J. Appl. Microbiol. Biotechnol., 1979, vol. 6, pp. 397-401. https://doi.org/10.1007/BF00499170

49. Du, L., Li, D., Zhu, T., Cai, S., Wang, F., Xiao, X., and Gu, Q., Tetrahedron, 2009, vol. 65, pp. 10331039. https://doi.org/10.1016/j.tet.2008.11.078

50. Kawai, K., Nozawa, K., Nakajima, S., and Iitaka, Y., Chem. Pharm. Bull., 1984, vol. 32, pp. 94-98. https://doi.org/10.1248/cpb.32.94

51. Yang, B., Sun, W., Wang, J., Lin, S., Li, X.-N., Zhu, H., Luo, Z., Xue, Y., Hu, Z., and Zhang, Y., Mar. Drugs, 2018, vol. 16, p. 110. https://doi.org/10.3390/md16040110

52. Du, L., Yang, X., Zhu, T., Wang, F., Xiao, X., Park, H., and Gu, Q., Chem. Pharm. Bull., 2009, vol. 57, pp. 873-876.

https://doi.org/10.1248/cpb.57.873

53. Zhong, W., Wang, J., Wei, X., Chen, Y., Fu, T., Xiang, Y., Huang, X., Tian, X., Xiao, Z., Zhang, W., Zhang, S., Long, L., and Wang, F., Org. Lett., 2018, vol. 20, pp. 4593-4596. https://doi.org/10.1021/acs.orglett.8b01880

54. Wang, W., Wan, X., Liu, J., Wang, J., Zhu, H., Chen, C., and Zhang, Y., Mar. Drugs, 2018, vol. 16, p. 150. https://doi.org/10.3390/md16050150

55. Zhang, M., Wang, W.-L., Fang, Y.-C., Zhu, T.-J., Gu, Q.-Q., and Zhu, W.-M., J. Nat. Prod., 2008, vol. 71, pp. 985-989.

https://doi.org/10.1021/np700737g

56. Singh, V.P., Yedukondalu, N., Sharma, V., Kushwaha, M., Sharma, R., Chaubey, A., Kumar, A., Singh, D., and Vishwakarma, R.A., J. Nat. Prod., 2018, vol. 81, pp. 219-226.

https://doi.org/10.1021/acs.jnatprod.6b00873

57. Rogozhin, E., Sadykova, V., Baranova, A., Vasilchenko, A., Lushpa, V., Mineev, K., Georgieva, M., Kul'ko, A., Krasheninnikov, M., Lyundup, A., Vasilchenko, A., and Andreev, Y., Molecules, 2018, vol. 23, 2785.

https://doi.org/10.3390/molecules23112785

58. Fdhila, F., Vázquez, V., Sánchez, J.L., and Riguera, R., J. Nat. Prod., 2003, vol. 66, pp. 1299-1301. https://doi.org/10.1021/np030233e
59. Guo, C., Lin, X.-P., Liao, S.-R., Yang, B., Zhou, X.-F., Yang, X.-W., Tian, X.-P., Wang, J.-F., and Liu, Y.-H., Nat. Prod. Res., 2020, vol. 34, pp. 1197-1205. https://doi.org/10.1080/14786419.2018.1553880

60. Zhao, D.-L., Wang, D., Tian, X.-Y., Cao, F., Li, Y.-Q., and Zhang, C.-S., Mar. Drugs, 2018, vol. 16, 36. https://doi.org/10.3390/md16010036

61. Wang, W., Chen, R., Luo, Z., Wang, W., and Chen, J., Nat. Prod. Res., 2018, vol. 32, pp. 558-563. https://doi.org/10.1080/14786419.2017.1329732

62. Bode, H.B., Bethe, B., Höfs, R., and Zeeck, A., ChemBioChem, 2002, vol. 3, pp. 619-627. https://doi.org/10.1002/1439-7633(20020703)3:7<619: :AID-CBIC619>3.0.CO;2-9

63. Yang, S.-Q., Li, X.-M., Xu, G.-M., Li, X., An, C.-Y., and Wang, B.-G., J. Antibiot., 2018, vol. 71, pp. 778784. https://doi.org/10.1038/s41429-018-0063-X

64. Hatsuda, Y., Hamasaki, T., Ishida, M., and Yoshikawa, S., Agric. Biol. Chem., 1969, vol. 33, pp. 131-133. https://doi.org/10.1080/00021369.1969.10859290

65. Endale, M., Ekberg, A., Alao, J., Akala, H., Ndakala, A., Sunnerhagen, P., Erdelyi, M., and Yenesew, A., Molecules, 2013, vol. 18, pp. 311-321. https://doi.org/10.3390/molecules18010311

66. Chung, M.-I., Jou, S.-J., Cheng, T.-H., Lin, C.-N., Ko, F.-N., and Teng, C.-M., J. Nat. Prod., 1994, vol. 57, pp. $313-316$. https://doi.org/10.1021/np50104a020

67. Moon, M.K., Han, Y.-M., Lee, Y.-J., Lee, L.H., Yang, J.H., Kwon, B.-M., and Kim, D.K., Arch. Pharm. Res., 2010, vol. 33, pp. 1747-1751. https://doi.org/10.1007/s12272-010-1106-4

68. Yan, L., Sen', Z., Li, Zh.-Kh., and Pan', I.-M., Khim. Prir. Soed., 2016, vol. 52, no. 5, pp. 760-761.

69. Wells, J.M., Cole, R.J., and Kirksey, J.W., Appl. Microbiol., 1975, vol. 30, pp. 26-28.

70. Liu, D., Yan, L., Ma, L., Huang, Y., Pan, X., Liu, W., and Lv, Z., Arch. Pharm. Res., 2015, vol. 38, pp. 10381043.

https://doi.org/10.1007/s12272-014-0371-z

71. May, Zin W.W., Buttachon, S., Dethoup, T., Pereira, J.A., Gales, L., Inácio, Â., Costa, P.M., Lee, M., Sekeroglu, N., Silva, A.M.S., Pinto, M.M.M., and Kijjoa, A., Phytochemistry, 2017, vol. 141, pp. 86-97. https://doi.org/10.1016/j.phytochem.2017.05.015

72. Hawas, U.W., El-Beih, A.A., and El-Halawany, A.M., Arch. Pharm. Res., 2012, vol. 35, pp. 1749-1756. https://doi.org/10.1007/s12272-012-1006-X

73. Orfali, R. and Perveen, S., J. Chem., 2019, vol. 2019, 7162948. https://doi.org/10.1155/2019/7162948

74. Said, G., Khou, Sh.-M., Lyu, Sh., Chao, Zh., Dzyan, Ya.-Ya., Zhen, Dzh.-E., and Shao, Ch.-L., Khim. Prir. Soed., 2019, vol. 55, no. 3, pp. 457-459.

75. Song, Z., Liu, Y., Gao, J., Hu, J., He, H., Dai, S., Wang, L., Dai, H., Zhang, L., and Song, F., Nat. Prod. Res., 2019. https://doi.org/10.1080/14786419.2019.1660331

76. Fang, W., Wang, J., Wang, J., Shi, L., Li, K., Lin, X., Min, Y., Yang, B., Tang, L., Liu, Y., and Zhou, X., J. 
Nat. Prod., 2018, vol. 81, pp. 1405-1410. https://doi.org/10.1021/acs.jnatprod.8b00015

77. Wang, W., Gao, M., Luo, Z., Liao, Y., Zhang, B., Ke, W., Shao, Z., Li, F., and Chen, J., Nat. Prod. Res., 2019, vol. 33, pp. 3077-3082. https://doi.org/10.1080/14786419.2018.1519561

78. Song, T., Chen, M., Chai, W., Zhang, Z., and Lian, X.-Y., Tetrahedron, 2018, vol. 74, pp. 884-891. https://doi.org/10.1016/j.tet.2018.01.015

79. Buttachon, S., Ramos, A., Inácio, Â., Dethoup, T., Gales, L., Lee, M., Costa, P., Silva, A., Sekeroglu, N., Rocha, E., Pinto, M., Pereira, J., and Kijjoa, A., Mar. Drugs, 2018, vol. 16, p. 119. https://doi.org/10.3390/md16040119

80. Danielsen, K., Aksnes, D.W., and Francis, G.W., Magn. Reson. Chem., 1992, vol. 30, pp. 359-360. https://doi.org/10.1002/mrc.1260300414

81. Lee, M.S., Cha, E.Y., Sul, J.Y., Song, I.S., and Kim, J.Y., Phytother. Res., 2011, vol. 25, pp. 833-837. https://doi.org/10.1002/ptr.3323

82. Semple, S.J., Pyke, S.M., Reynolds, G.D., and Flower, R.L.P., Antiviral Res., 2001, vol. 49, pp. 169-178. https://doi.org/10.1016/S0166-3542(01)00125-5

83. Li, J.L., Jiang, X., Liu, X., He, C., Di, Y., Lu, S., Huang, H., Lin, B., Wang, D., and Fan, B., Fitoterapia, 2019, vol. 133, pp. 1-4. https://doi.org/10.1016/j.fitote.2018.11.015

84. Lee, Y.M., Li, H., Hong, J., Cho, H.Y., Bae, K.S., Kim, M.A., Kim, D.-K., and Jung, J.H., Arch. Pharm. Res., 2010, vol. 33, pp. 231-235. https://doi.org/10.1007/s12272-010-0207-4

85. Liu, K., Zheng, Y., Miao, C., Xiong, Z., Xu, L., Guan, H., Yang, Y., and Zhao, L., Nat. Prod. Res., 2014, vol. 28, pp. 2334-2337. https://doi.org/10.1080/14786419.2014.935941

86. Sedmera, P., Volc, J., Weijer, J., Vokoun, J., and Musilek, V., Collect. Czech. Chem. Commun., 1981, vol. 46, pp. $1210-1216$. https://doi.org/10.1135/cccc19811210

87. Pang, X., Lin, X., Wang, P., Zhou, X., Yang, B., Wang, J., and Liu, Y., Mar. Drugs, 2018, vol. 16, 280. https://doi.org/10.3390/md16080280

88. Zhao, D.-L., Cao, F., Wang, C.-Y., Yang, L.-J., Shi, T., Wang, K.-L., Shao, C.-L., and Wang, C.-Y., J. Nat. Prod., 2019, vol. 82, pp. 3201-3204. https://doi.org/10.1021/acs.jnatprod.9b00905

89. Boutin, B., Peeler, J., and Twedt, R., J. Toxicol. Environ. Health, 1989, vol. 26, pp. 75-81. https://doi.org/10.1080/15287398909531234

90. Gao, S.-S., Li, X.-M., and Wang, B.-G., Nat. Prod. Commun., 2009, vol. 4, p. 1477-1480. https://doi.org/10.1177/1934578X0900401107

91. Okuno, T., Natsume, I., Sawai, K., Sawamura, K., Furusaki, A., and Matsumoto, T., Tetrahedron Lett., 1983, vol. 24, pp. 5653-5656. https://doi.org/10.1016/S0040-4039(00)94165-0

92. Kornsakulkarn, J., Dolsophon, K., Boonyuen, N., Boonruangprapa, T., Rachtawee, P., Prabpai, S., Kongsaeree, P., and Thongpanchang, C., Tetrahedron, 2011, vol. 67, pp. 7540-7547.

https://doi.org/10.1016/j.tet.2011.07.078
93. Supratman, U., Hirai, N., Sato, S., Watanabe, K., Malik, A., Annas, S., Harneti, D., Maharani, R., Koseki, T., and Shiono, Y., Nat. Prod. Res., 2019. https://doi.org/10.1080/14786419.2019.1650358

94. Li, Q., Zhu, R., Yi, W., Chai, W., Zhang, Z., and Lian, X.-Y., Phytochemistry, 2018, vol. 152, pp. 53-60. https://doi.org/10.1016/j.phytochem.2018.04.021

95. Bashyal, B.P., Wellensiek, B.P., Ramakrishnan, R., Faeth, S.H., Ahmad, N., and Gunatilaka, A.A.L., Bioorg. Med. Chem., 2014, vol. 22, pp. 6112-6116. https://doi.org/10.1016/j.bmc.2014.08.039

96. Zhu, A., Zhang, X.-W., Zhang, M., Li, W., Ma, Z.-Y., Zhu, H.-J., and Cao, F., Mar. Drugs, 2018, vol. 16, p. 312 . https://doi.org/10.3390/md16090312

97. Zhu, A., Yang, M.-Y., Zhang, Y.-H., Shao, C.-L., Wang, C.-Y., Hu, L.-D., Cao, F., and Zhu, H.-J., Sci. Rep., 2018, vol. 8, p. 10621. https://doi.org/10.1038/s41598-018-28996-5

98. González-Andrade, M., Rivera-Chávez, J., SosaPeinado, A., Figueroa, M., Rodríguez-Sotres, R., and Mata, R., J. Med. Chem., 2011, vol. 54, pp. 3875-3884. https://doi.org/10.1021/jm200167g

99. Figueroa, M., González, M., Rodríguez-Sotres, R., Sosa-Peinado, A., González-Andrade, M., CerdaGarcía-Rojas, C.M., and Mata, R, Bioorg. Med. Chem., 2009, vol. 17, pp. 2167-2174. https://doi.org/10.1016/j.bmc.2008.10.079

100. Song, Z., Gao, J., Hu, J., He, H., Huang, P., Zhang, L., and Song, F., Nat. Prod. Res., 2020, vol. 34, pp. 29072912. https://doi.org/10.1080/14786419.2019.1597355

101. Maes, C.M. and Steyn, P.S., J. Chem. Soc., Perkin Trans., 1984, pp. 1137-1140. https://doi.org/10.1039/p19840001137

102. Putra, H.N., Rukachaisirikul, V., Saithong, S., Phongpaichit, S., Preedanon, S., Sakayaroj, J., Hadsadee, S., and Jungsuttiwong, S., Tetrahedron, 2019, vol. 75 , p. 130530. https://doi.org/10.1016/j.tet.2019.130530

103. Takahashi, I., Kawano, T., and Kimura, Y., Z. Naturforsch., 2001, vol. 56b, pp. 797-803. https://doi.org/10.1515/znb-2001-0813

104. Hamasaki, T., Sato, Y., and Hatsuda, Y., Agric. Biol. Chem., 1975, vol. 39, pp. 2341-2345. https://doi.org/10.1080/00021369.1975.10861955

105. Sun, R.-R., Miao, F.-P., Zhang, J., Wang, G., Yin, X.-L., and Ji, N.-Y., Magn. Reson. Chem., 2013, vol. 51, pp. $65-68$. https://doi.org/10.1002/mrc.3903

106. Trisuwan, K., Rukachaisirikul, V., Kaewpet, M., Phongpaichit, S., Hutadilok-Towatana, N., Preedanon, S., and Sakayaroj, J., J. Nat. Prod., 2011, vol. 74, pp. $1663-1667$. https://doi.org/10.1021/np200374j

107. Elnaggar, M.S., Ebada, S.S., Ashour, M.L., Ebrahim, W., Müller, W.E.G., Mándi, A., Kurtán, T., Singab, A., Lin, W., Liu, Z., and Proksch, P., Tetrahedron, 2016, vol. 72, pp. 2411-2419. https://doi.org/10.1016/j.tet.2016.03.073

108. Salendra, L., Lin, X., Chen, W., Pang, X., Luo, X., Long, J., Liao, S., Wang, J., Zhou, X., Liu, Y., and 
Yang, B., Nat. Prod. Res., 2019. https://doi.org/10.1080/14786419.2019.1610757

109. Wang, H., Gloer, J.B., Scott, J.A., and Malloch, D., Tetrahedron Lett., 1995, vol. 36, pp. 5847-5850. https://doi.org/10.1016/0040-4039(95)01174-G

110. Khamthong, N., Rukachaisirikul, V., Phongpaichit, S., Preedanon, S., and Sakayaroj, J., Tetrahedron, 2012, vol. 68 , pp. $8245-8250$.

https://doi.org/10.1016/j.tet.2012.07.060

111. Sun, Y.-L., Bao, J., Liu, K.-S., Zhang, X.-Y., He, F., Wang, Y.-F., Nong, X.-H., and Qi, S.-H., Planta Med., 2013, vol. 79, pp. 1474-1479.

https://doi.org/10.1055/s-0033-1350805

112. Li, X., Zhang, L., Liu, Y., Guo, Z., Deng, Z., Chen, J., and Xuantu Zou, K., Nat. Prod. Commun., 2013, vol. 8, p. 1934578X1300800. https://doi.org/10.1177/1934578X1300800510

113. Luo, Y.-P., Zheng, C.-J., Chen, G.-Y., Song, X.-P., and Wang, Z., J. Antibiot., 2019, vol. 72, pp. 513-517. https://doi.org/10.1038/s41429-019-0178-8

114. Kumla, D., Pereira, J., Dethoup, T., Gales, L., FreitasSilva, J., Costa, P., Lee, M., Silva, A., Sekeroglu, N., Pinto, M., and Kijjoa, A., Mar. Drugs, 2018, vol. 16, 289.

https://doi.org/10.3390/md16080289

115. Fujita, K.-I., Nagamine, Y., Ping, X., and Taniguchi, M., J. Antibiot., 1999, vol. 52, pp. 628-634. https://doi.org/10.7164/antibiotics.52.628

116. Wang, L., Han, X., Zhu, G., Wang, Y., Chairoungdua, A., Piyachaturawat, P., and Zhu, W., Front. Chem., 2018, vol. 6,344 . https://doi.org/10.3389/fchem.2018.00344

117. Zhao, G.-Y., Fan, J.-Y., Hua, C.-P., Yan, W., Chen, C.-J., Lu, Y.-H., Jiao, R.-H., and Tan, R.-X., RSC Adv., 2015, vol. 5, pp. 5657-5663. https://doi.org/10.1039/C4RA12851F

118. Chen, L., Li, Y.-P., Li, X.-X., Lu, Z.-H., Zheng, Q.-H., and Liu, Q.-Y., J. Antibiot., 2019, vol. 72, pp. 34-44. https://doi.org/10.1038/s41429-018-0104-5

119. Ving, L. and Mcinnes, A., Secalonic Acids and Their Use, DE 3002761, 1980.

120. Zhang, J., Tao, L., Liang, Y., Yan, Y., Dai, C., Xia, X., She, Z., Lin, Y., and Fu, L., Cell Cycle, 2009, vol. 8, pp. 2444-2450.

https://doi.org/10.4161/cc.8.15.9170

121. Bai, M., Zheng, C.-J., Tang, D.-Q., Zhang, F., Wang, H.-Y., and Chen, G.-Y., J. Antibiot., 2019, vol. 72, pp. 779-782.

https://doi.org/10.1038/s41429-019-0206-8

122. Liu, F., Cai, X.-L., Yang, H., Xia, X.-K., Guo, Z.-Y., Yuan, J., Li, M.-F., She, Z.-G., and Lin, Y.-C., Planta Med., 2010, vol. 76, pp. 185-189. https://doi.org/10.1055/s-0029-1186047

123. Lenta, B.N., Ngatchou, J., Frese, M., Ladoh-Yemeda, F., Voundi, S., Nardella, F., Michalek, C., Wibberg, D., Ngouela, S., Tsamo, E., Kaiser, M., Kalinowski, J., and Sewald, N., Z. Naturforsch. B, 2016, vol. 71, pp. $1159-1167$.

https://doi.org/10.1515/znb-2016-0128

124. Zheng, C.-J., Liao, H.-X., Mei, R.-Q., Huang, G.-L., Yang, L.-J., Zhou, X.-M., Shao, T.-M., Chen, G.-Y., and Wang, C.-Y., Nat. Prod. Res., 2019, vol. 33, pp. $1127-1134$.

https://doi.org/10.1080/14786419.2018.1460832

125. Inamori, Y., Kato, Y., Kubo, M., Kamiki, T., Takemoto, T., and Nomoto, K., Chem. Pharm. Bull., 1983, vol. 31, pp. 4543-4548.

https://doi.org/10.1248/cpb.31.4543

126. Tao, Q., Ding, C., Auckloo, B.N., and Wu, B., Nat. Prod. Commun., 2018, vol. 13, pp. 571-573. https://doi.org/10.1177/1934578X1801300514

127. Lu, C.-H., Huang, Y.-J., and Shen, Y.-M., Chin. J. Nat. Med., 2005, vol. 3, pp. 269-271.

128. Ukwatta, K.M., Lawrence, J.L., and Wijayarathna, C.D., Mycology, 2019, vol. 10, pp. 222-228. https://doi.org/10.1080/21501203.2019.1620892

129. Capon, R.J., Stewart, M., Ratnayake, R., Lacey, E., and Gill, J.H., J. Nat. Prod., 2007, vol. 70, pp. 17461752. https://doi.org/10.1021/np0702483

130. He, F., Li, X., Yu, J.-H., Zhang, X., Nong, X., Chen, G., Zhu, K., Wang, Y.-Y., Bao, J., and Zhang, H., Fitoterapia, 2019, vol. 136, 104177. https://doi.org/10.1016/j.fitote.2019.104177

131. Bladt, T., Frisvad, J., Knudsen, P., and Larsen, T., Molecules, 2013, vol. 18, pp. 11338-11376. https://doi.org/10.3390/molecules 180911338

132. Xia, X.-K., Huang, H.-R., She, Z.-G., Cai, J.-W., Lan, L., Zhang, J.-Y., Fu, L.-W., Vrijmoed, L.L.P., and Lin, Y.-C., Helv. Chim. Acta, 2007, vol. 90, pp. 1925-1931. https://doi.org/10.1002/hlca.200790200

133. De Stefano, S., Nicoletti, R., Milone, A., and Zambardino, S., Phytochemistry, 1999, vol. 52, pp. 13991401. https://doi.org/10.1016/S0031-9422(99)00320-9

134. Stammati, A., Nicoletti, R., De Stefano, S., Zampaglioni, F., and Zucco, F., Altern. Lab. Anim., 2002, vol. 30, pp. 69-75. https://doi.org/10.1177/026119290203000107

135. Noor, A.O., Almasri, D.M., Bagalagel, A.A., Abdallah, H.M., Mohamed, S.G.A., Mohamed, G.A., and Ibrahim, S.R.M., Molecules, 2020, vol. 25, p. 395. https://doi.org/10.3390/molecules25020395

136. Chen, Y., Liu, Z., Liu, H., Pan, Y., Li, J., Liu, L., and She, Z., Mar. Drugs, 2018, vol. 16, p. 54. https://doi.org/10.3390/md16020054

137. Li, C., Gong, B., Cox, D., Li, C., Wang, J., and Ding, W., Pharmacogn. Mag., 2014, vol. 10, pp. 153-158. https://doi.org/10.4103/0973-1296.127367

138. Aly, A.H., Edrada-Ebel, R., Wray, V., Muller, W.E.G., Kozytska, S., Hentschel, U., Proksch, P., and Ebel, R., Phytochemistry, 2008, vol. 69, pp. 1716-1725. https://doi.org/10.1016/j.phytochem.2008.02.013

139. Wensheng, L., Jiaxin, X., Fenqi, L., Li, X., and Chunyuan, L., Pharmacogn. Mag., 2016, vol. 12, pp. $259-261$. https://doi.org/10.4103/0973-1296.192204

140. Larsen, T.O. and Breinholt, J., J. Nat. Prod., 1999, vol. 62, pp. $1182-1184$.

https://doi.org/10.1021/np990066b 
141. Xu, Z., Xiong, B., and Xu, J., Nat. Prod. Res., 2019. https://doi.org/10.1080/14786419.2019.1656624

142. Chen, S., Liu, Y., Liu, Z., Cai, R., Lu, Y., Huang, X., and She, Z., RSC Adv., 2016, vol. 6, pp. 26412-26420. https://doi.org/10.1039/C6RA02566H

143. Aly, A.H., Edrada-Ebel, R., Indriani, I.D., Wray, V., Muller, W.E.G., Totzke, F., Zirrgiebel, U., Schächtele, C., Kubbutat, M.H.G., Lin, W.H., Proksch, P., and Ebel, R., J. Nat. Prod., 2008, vol. 71, pp. 972-980. https://doi.org/10.1021/np070447m

144. Kim, N., Sohn, M.-J., Koshino, H., Kim, E.-H., and Kim, W.-G., Bioorg. Med. Chem. Lett., 2014, vol. 24, pp. 83-86. https://doi.org/10.1016/j.bmcl.2013.11.071

145. Xu, Z., Wu, X., Li, G., Feng, Z., and Xu, J., Nat. Prod. Res., 2020, vol. 34, pp. 1002-1007. https://doi.org/10.1080/14786419.2018.1539980

146. Wang, Y., Yang, M.-H., Wang, X.-B., Li, T.-X., and Kong, L.-Y., Fitoterapia, 2014, vol. 99, pp. 153-158. https://doi.org/10.1016/j.fitote.2014.09.015

147. Souza, G., De Mithöfer, A., Daolio, C., Schneider, B., and Rodrigues-Filho, E., Molecules, 2013, vol. 18, pp. $2528-2538$. https://doi.org/10.3390/molecules 18032528

148. Jiao, P., Gloer, J.B., Campbell, J., and Shearer, C.A., J. Nat. Prod., 2006, vol. 69, pp. 612-615. https://doi.org/10.1021/np0504661

149. Khan', Zh., Mei, V., Zhao, E., Den, Yu., and Dai, Kh., Khim. Prir. Soed., 2009, vol. 45, no. 6, pp. 676-677.

150. Wang, X., Radwan, M.M., Taráwneh, A.H., Gao, J., Wedge, D.E., Rosa, L.H., Cutler, H.G., and Cutler, S.J., J. Agricult. Food Chem., 2013, vol. 61, pp. 4551-4555. https://doi.org/10.1021/jf400212y

151. Afiyatullov, S.S., Zhuravleva, O.I., Antonov, A.S., Leshchenko, E.V., Pivkin, M.V., Khudyakova, Y.V., Denisenko, V.A., Pislyagin, E.A., Kim, N.Y., Berdyshev, D.V., von Amsberg, G., and Dyshlovoy, S.A., Mar. Drugs, 2019, vol. 17, 647. https://doi.org/10.3390/md17110647

152. Zhang, F.-Z., Li, X.-M., Yang, S.-Q., Meng, L.-H., and Wang, B.-G., J. Nat. Prod., 2019, vol. 82, pp. $1535-1541$.

https://doi.org/10.1021/acs.jnatprod.8b01091

153. Jadulco, R., Proksch, P., Wray, V., Sudarsono, Berg, A., and Gräfe, U., J. Nat. Prod., 2001, vol. 64, pp. 527530. https://doi.org/10.1021/np000401s

154. Grabley, S., Granzer, E., Hütter, K., Ludwig, D., Mayer, M., Thiericke, R., Till, G., Wink, J., Philipps, S., and Zeeck, A., J. Antibiot., 1992, vol. 45, pp. 56-65. https://doi.org/10.7164/antibiotics.45.56

155. An, F.-L., Liu, W.-H., Wei, X.-C., Pan, Z.-H., and Lu, Y.-H., Nat. Prod. Commun., 2018, vol. 13, pp. 865866. https://doi.org/10.1177/1934578X1801300720

156. Umeokoli, B.O., Ebrahim, W., El-Neketi, M., Müller, W.E.G., Kalscheuer, R., Lin, W., Liu, Z., and Proksch, P., Nat. Prod. Res., 2019, vol. 33, pp. 22152222.

https://doi.org/10.1080/14786419.2018.1496430
157. Abdou, R., Scherlach, K., Dahse, H.-M., Sattler, I., and Hertweck, C., Phytochemistry, 2010, vol. 71, pp. 110-116.

158. Aldridge, D.C., Galt, S., Giles, D., and Turner, W.B., J. Chem. Soc. C: Org., 1971, pp. 1623-1627. https://doi.org/10.1039/j39710001623

159. Xia, M.-W., Yang, Y., Xu, R., Li, C.-W., and Cui, C.-B., Nat. Prod. Res., 2019, vol. 33, pp. 89-94. https://doi.org/10.1080/14786419.2018.1434644

160. Carvalho, M.R., Barbosa, L.C., Queiróz, J.H., and Howarth, O.W., Tetrahedron Lett., 2001, vol. 42, pp. 809-811. https://doi.org/10.1016/S0040-4039(00)02154-7

161. Zhao, D.-L., Shao, C.-L., Zhang, Q., Wang, K.-L., Guan, F.-F., Shi, T., and Wang, C.-Y., J. Nat. Prod., 2015, vol. 78, pp. 2310-2314. https://doi.org/10.1021/acs.jnatprod.5b00575

162. Hayasaka, S., Koseki, T., Murayama, T., Kwon, E., and Shiono, Y., Z. Naturforsch. B, 2011, vol. 66, pp. 961-964.

https://doi.org/10.1515/znb-2011-0915

163. Yurchenko, A.N., Smetanina, O.F., Kalinovskii, A.I., Pivkin, M.V., Dmitrenok, P.S., and Kuznetsova, T.A., Izv. Akad. Nauk, Ser. Khim., 2010, vol. 59, no. 4, pp. 834-838.

164. Chokpaiboon, S., Unagul, P., Nithithanasilp, S., Komwijit, S., Somyong, W., Ratiarpakul, T., Isaka, M., and Bunyapaiboonsri, T., Nat. Prod. Res., 2018, vol. 32 , pp. 149-153. https://doi.org/10.1080/14786419.2017.1342083

165. Zheng, C.-J., Huang, G.-L., Liao, H.-X., Mei, R.-Q., Luo, Y.-P., Chen, G.-Y., and Zhang, Q.-Y., Bioorg. Chem., 2019, vol. 85, pp. 382-385. https://doi.org/10.1016/j.bioorg.2019.01.015

166. Khuan, Zh., Guo, Zh., Yan, Zh., Iin, S., Li, S., Luo, V., She, Zh., and Lin', E., Khim. Prir. Soed., 2009, vol. 45, no. 5, pp. 528-530.

167. Kornsakulkarn, J., Somyong, W., Supothina, S., Boonyuen, N., and Thongpanchang, C., Tetrahedron, 2015, vol. 71, pp. 9112-9116.

https://doi.org/10.1016/j.tet.2015.10.015

168. Beekman, A.M. and Barrow, R.A., Aust. J. Chem., 2015, vol. 68, pp. 1583-1592.

https://doi.org/10.1071/CH15144

169. Matsuo, H., Nonaka, K., Nagano, Y., Yabuki, A., Fujikura, K., Takahashi, Y., Omura, S., and Nakashima, T., Biosci. Biotechnol. Biochem., 2018, vol. 82, pp. 13231326. https://doi.org/10.1080/09168451.2018.1467264

170. Elissawy, A.M., Ebada, S.S., Ashour, M.L., El-Neketi, M., Ebrahim, W., and Singab, A.B., Phytochem. Lett., 2019, vol. 29, pp. 1-5. https://doi.org/10.1016/j.phytol.2018.10.014

171. Ishikawa, Y., Morimoto, K., and Hamasaki, T., J. Am. Oil Chem. Soc., 1984, vol. 61, pp. 1864-1868. https://doi.org/10.1007/BF02540819

172. Zhang, Z., He, X., Liu, C., Che, Q., Zhu, T., Gu, Q., and Li, D., RSC Adv., 2016, vol. 6, pp. 76498-76504. https://doi.org/10.1039/C6RA14640F

173. Li, H.-L., Li, X.-M., Mandi, A., Antus, S., Li, X., Zhang, P., Liu, Y., Kurtán, T., and Wang, B.-G., J. 
Org. Chem., 2017, vol. 82, pp. 9946-9954. https://doi.org/10.1021/acs.joc.7b01277

174. Reichling, J., Suschke, U., Schneele, J., and Geiss, H.K., Nat. Prod. Commun., 2006, vol. 1, pp. 1003-1012. https://doi.org/10.1177/1934578X0600101116

175. Reddy, M., Gupta, S., Jacob, M., Khan, S., and Ferreira, D., Planta Med., 2007, vol. 73, pp. 461-467. https://doi.org/10.1055/s-2007-967167

176. Frank, M., Hartmann, R., Plenker, M., Mandi, A., Kurtán, T., Özkaya, F.C., Muller, W.E.G., Kassack, M.U., Hamacher, A., Lin, W., Liu, Z., and Proksch, P., J. Nat. Prod., 2019, vol. 82, pp. 21592166. https://doi.org/10.1021/acs.jnatprod.9b00151

177. Klaiklay, S., Rukachaisirikul, V., Tadpetch, K., Sukpondma, Y., Phongpaichit, S., Buatong, J., and Sakayaroj, J., Tetrahedron, 2012, vol. 68, pp. 2299-2305. https://doi.org/10.1016/j.tet.2012.01.041

178. Itabashi, T., Nozawa, K., Nakajima, S., and Kawai, K., Chem. Pharm. Bull., 1993, vol. 41, pp. 2040-2041. https://doi.org/10.1248/cpb.41.2040

179. Taniguchi, M., Kaneda, N., Shibata, K., and Kamikawa, T., Agricult. Biol. Chem., 1978, vol. 42, pp. 16291630. https://doi.org/10.1080/00021369.1978.10863223

180. Nuankeaw, K., Chaiyosang, B., Suebrasri, T., Kanokmedhakul, S., Lumyong, S., and Boonlue, S., Mycoscience, 2020, vol. 61, pp. 16-21.

https://doi.org/10.1016/j.myc.2019.10.001

181. Yamazaki, M. and Maebayashi, Y., Chem. Pharm. Bull., 1982, vol. 30, pp. 514-518. https://doi.org/10.1248/cpb.30.514

182. Chomcheon, P., Wiyakrutta, S., Sriubolmas, N., Ngamrojanavanich, N., Mahidol, C., Ruchirawat, S., and Kittakoop, P., Phytochemistry, 2009, vol. 70, pp. $121-127$.

https://doi.org/10.1016/j.phytochem.2008.10.007

183. Fang, M.J., Fang, H., Li, W.J., Huang, D.M., Wu, Z., and Zhao, Y.F., Nat. Prod. Res., 2012, vol. 26, pp. 1224-1228. https://doi.org/10.1080/14786419.2011.559947

184. Hargreaves, J., Park, J., Ghisalberti, E.L., Sivasithamparam, K., Skelton, B.W., and White, A.H., J. Nat. Prod., 2002, vol. 65, pp. 7-10. https://doi.org/10.1021/np0102758

185. Fang, S.-T., Wang, Y.-J., Ma, X.-Y., Yin, X.-L., and Ji, N.-Y., Nat. Prod. Res., 2019, vol. 33, pp. 3127-3133. https://doi.org/10.1080/14786419.2018.1522314

186. Wen, S., Fan, W., Guo, H., Huang, C., Yan, Z., and Long, Y., Nat. Prod. Res., 2020, vol. 34, pp. 2919-2925. https://doi.org/10.1080/14786419.2019.1598993

187. Xu, X., Yang, H., Xu, H., Yin, L., Chen, Z., and Shen, H., Nat. Prod. Res., 2018, vol. 32, pp. 821-825. https://doi.org/10.1080/14786419.2017.1363754

188. Gao, H., Zhou, L., Cai, S., Zhang, G., Zhu, T., Gu, Q., and Li, D., J. Antibiot., 2013, vol. 66, pp. 539-542. https://doi.org/10.1038/ja.2013.40

189. Li, X.-B., Zhou, Y.-H., Zhu, R.-X., Chang, W.-Q., Yuan, H.-Q., Gao, W., Zhang, L.-L., Zhao, Z.-T., and Lou, H.-X., Chem. Biodiversity, 2015, vol. 12, pp. 575592.

https://doi.org/10.1002/cbdv.201400146
190. Cooke, R.G. and Edwards, J.M., Fortschritte der Chemie organischer Naturstoffe (Progress in the Chemistry of Organic Natural Products), Herz, W., Grisebach, H., and Kirby, G.W., Eds., Vienna: Springer Vienna, 1981, pp. $153-190$. https://doi.org/10.1007/978-3-7091-8611-4_4

191. Homma, K., Fukuyama, K., Katsube, Y., Kimura, Y., and Hamasaki, T., Agric. Biol. Chem., 1980, vol. 44, pp. $1333-1337$. https://doi.org/10.1271/bbb1961.44.1333

192. Ayer, W.A. and Hiratsuka, Y., Rev. Latinoam. Quim., 1982, vol. 13, pp. 84-87.

193. Ayer, W.A., Hoyano, Y., Pedras, M.S., and van Altena, I., Can. J. Chem., 1986, vol. 64, pp. 1585-1589. https://doi.org/10.1139/v86-262

194. Tanaka, Y., Matsuzaki, K., Zhong, C.-L., Yoshida, H., Kawakubo, T., Masuma, R., Tanaka, H., and Omura, S., J. Antibiot., 1996, vol. 49, pp. 1056-1059. https://doi.org/10.7164/antibiotics.49.1056

195. Bai, Y.-B., Gao, Y.-Q., Nie, X.-D., Tuong, T.-M.-L., Li, D., and Gao, J.-M., J. Agricult. Food Chem., 2019, vol. 67, pp. 6125-6132. https://doi.org/10.1021/acs.jafc.9b00606

196. Gauthier, T., Wang, X., Sifuentes, Dos., Santos, J., Fysikopoulos, A., Tadrist, S., Canlet, C., Artigot, M.P., Loiseau, N., Oswald, I.P., and Puel, O., PLoS One, 2012, vol. 7, e29906. https://doi.org/10.1371/journal.pone.0029906

197. Gao, J.-M., Yang, S.-X., and Qin, J.-C., Chem. Rev., 2013, vol. 113, pp. 4755-4811. https://doi.org/10.1021/cr300402y

198. Wang, C.-Y., Hao, J.-D., Ning, X.-Y., Wu, J.-S., Zhao, D.-L., Kong, C.-J., Shao, C.-L., and Wang, C.-Y., $R S C A d v .$, 2018, vol. 8, pp. 4348-4353. https://doi.org/10.1039/C7RA13327H

199. Pairet, L., Wrigley, S.K., Chetland, I., Reynolds, E.E., Hayes, M.A., Holloway, J., Ainsworth, A.M., Katzer, W., Cheng, X.-M., Hupe, D.J., Charlton, P., and Doherty, A.M., J. Antibiot., 1995, vol. 48, pp. 913-923. https://doi.org/10.7164/antibiotics.48.913

200. Luo, X., Lin, X., Tao, H., Wang, J., Li, J., Yang, B., Zhou, X., and Liu, Y., J. Nat. Prod., 2018, vol. 81, pp. 934-941. https://doi.org/10.1021/acs.jnatprod.7b01053

201. Hemtasin, C., Kanokmedhakul, S., Moosophon, P., Soytong, K., and Kanokmedhakul, K., Phytochem. Lett., 2016, vol. 16, pp. 56-60. https://doi.org/10.1016/j.phytol.2016.03.004

202. Myobatake, Y., Takeuchi, T., Kuramochi, K., Kuriyama, I., Ishido, T., Hirano, K., Sugawara, F., Yoshida, H., and Mizushina, Y., J. Nat. Prod., 2012, vol. 75, pp. $135-141$. https://doi.org/10.1021/np200523b

203. Yang, S.-W., Chan, T.-M., Terracciano, J., Patel, R., Patel, M., Gullo, V., and Chu, M., J. Antibiot., 2006, vol. 59, pp. 720-723. https://doi.org/10.1038/ja.2006.96

204. Trivedi, A.B., Hirota, M., Doi, E., and Kitabatake, N., J. Chem. Soc., Perkin Trans. 1, 1993, pp. 2167-2171. https://doi.org/10.1039/p19930002167

205. Sadorn, K., Saepua, S., Boonyuen, N., Laksanacharoen, P., Rachtawee, P., and Pittayakhajonwut, P., 
RSC Adv., 2016, vol. 6, pp. 94510-94523.

https://doi.org/10.1039/C6RA21898A

206. Chen, S., Wang, J., Wang, Z., Lin, X., Zhao, B., Kaliaperumal, K., Liao, X., Tu, Z., Li, J., Xu, S., and Liu, Y., Fitoterapia, 2017, vol. 117, pp. 71-78. https://doi.org/10.1016/j.fitote.2017.01.005

207. Vanmiddlesworth, F., Giacobbe, R.A., Lopez, M., Garrity, G., Bland, J.A., Bartizal, K., Fromtling, R.A., Polishook, J., Zweerink, M., Edison, A.M., Rozdilsky, W., Wilson, K.E., and Monaghan, R.L., J. Antibiot., 1992, vol. 45, pp. 861-867. https://doi.org/10.7164/antibiotics.45.861

208. Choi, B.-K., Phan, T.H.T., Hwang, S., Oh, D.-C., Kang, J.S., Lee, H.-S., Ngo, T.D.N., Tran, T.T.V., and Shin, H.J., J. Nat. Prod., 2019, vol. 82, pp. 31863190. https://doi.org/10.1021/acs.jnatprod.9b00776

209. Neuhaus, G.F., Adpressa, D.A., Bruhn, T., and Loesgen, S., J. Nat. Prod., 2019, vol. 82, pp. 2780-2789. https://doi.org/10.1021/acs.jnatprod.9b00416

210. Sassa, T., Takemura, T., Ikeda, M., and Miura, Y., Tetrahedron Lett., 1973, vol. 14, pp. 2333-2334. https://doi.org/10.1016/S0040-4039(01)96211-2

211. Niu, S., Tang, X.-X., Fan, Z., Xia, J.-M., Xie, C.-L., and Yang, X.-W., Mar. Drugs, 2019, vol. 17, 125. https://doi.org/10.3390/md17020125

212. Höller, U., Wright, A.D., Matthee, G.F., Konig, G.M., Draeger, S., Aust, H.-J., and Schulz, B., Mycol. Res., 2000, vol. 104, pp. 1354-1365. https://doi.org/10.1017/S0953756200003117

213. Yu, G., Sun, Z., Peng, J., Zhu, M., Che, Q., Zhang, G., Zhu, T., Gu, Q., and Li, D., J. Nat. Prod., 2019, vol. 82, pp. 2013-2017. https://doi.org/10.1021/acs.jnatprod.9b00345

214. Kanoh, K., Adachi, K., Matsuda, S., Shizuri, Y., Yasumoto, K., Kusumi, T., Okumura, K., and Kirikae, T., J. Antibiot., 2008, vol. 61, pp. 192-194. https://doi.org/10.1038/ja.2008.29

215. Madhyastha, M.S., Marquardt, R.R., Masi, A., Borsa, J., and Frohlich, A.A., J. Food Protect., 1994, vol. 57, pp. $48-53$. https://doi.org/10.1038/ja.2008.29

216. Al-Fakih, A.A. and Almaqtri, W.Q.A., Mycology, 2019, vol. 10, pp. 191-209. https://doi.org/10.1080/21501203.2019.1604576

217. Vansteelandt, M., Blanchet, E., Egorov, M., Petit, F., Toupet, L., Bondon, A., Monteau, F., Le Bizec, B., Thomas, O.P., Pouchus, Y.F., Le Bot, R., and Grovel, O., J. Nat. Prod., 2013, vol. 76, pp. 297-301. https://doi.org/10.1021/np3007364

218. Nguyen, H.T., Yu, N.H., Jeon, S.J., Lee, H.W., Bae, C.-H., Yeo, J.H., Lee, H.B., Kim, I.-S., Park, H.W., and Kim, J.-C., Lett. Appl. Microbiol., 2016, vol. 62, pp. 488-493. https://doi.org/10.1111/lam.12578

219. Liu, W.-H., Ding, Y., Ji, X., An, F.-L., and Lu, Y.-H., J. Antibiot., 2019, vol. 72, pp. 111-113. https://doi.org/10.1038/s41429-018-0110-7

220. Zhu, G., Kong, F., Wang, Y., Fu, P., and Zhu, W., Mar. Drugs, 2018, vol. 16, 71. https://doi.org/10.3390/md16020071
221. Li, M.-F., Li, G.-H., and Zhang, K.-Q., Metabolites, 2019, vol. 9, 58. https://doi.org/10.3390/metabo9030058

222. Corral, P., Esposito, F.P., Tedesco, P., Falco, A., Tortorella, E., Tartaglione, L., Festa, C., D’Auria, M.V., Gnavi, G., Varese, G.C., and De Pascale, D., Mar. Biotechnol., 2018, vol. 20, pp. 502-511. https://doi.org/10.1007/s10126-018-9821-9

223. Trifonov, L.S., Hilpert, H., Floersheim, P., Dreiding, A.S., Rast, D.M., Skrivanova, R., and Hoesch, L., Tetrahedron, 1986, vol. 42, pp. 3157-3179. https://doi.org/10.1016/S0040-4020(01)87382-8

224. Fujii, Y., Asahara, M., Ichinoe, M., and Nakajima, H., Phytochemistry, 2002, vol. 60, pp. 703-708. https://doi.org/10.1016/S0031-9422(02)00196-6

225. Won-Gon, K., Ryoo, I.-J., Park, S.-H., Kim, D.-S., Lee, S., Park, K.-C., and Yoo, I.-D., J. Microbiol. Biotechnol., 2005, vol. 15, pp. 891-894.

226. Asfour, H.Z., Awan, Z.A., Bagalagel, A.A., Elfaky, M.A., Abdelhameed, R.F.A., and Elhady, S.S., Biomolecules, 2019, vol. 9, 480 . https://doi.org/10.3390/biom9090480

227. Tan, Q.-W., Fang, P.-H., Ni, J.-C., Gao, F., and Chen, Q.-J., Molecules, 2017, vol. 22, 2073. https://doi.org/10.3390/molecules22122073

228. Pastre, R., Marinho, A.M.R., Rodrigues-Filho, E., Souza, A.Q.L., and Pereira, J.O., Química Nova, 2007, vol. 30, pp. 1867-1871. https://doi.org/10.1590/S0100-40422007000800013

229. Song, T., Chen, M., Ge, Z.-W., Chai, W., Li, X.-C., Zhang, Z., and Lian, X.-Y., J. Org. Chem., 2018, vol. 83, pp. 13395-13401.

https://doi.org/10.1021/acs.joc.8b02172

230. Rukachaisirikul, V., Satpradit, S., Klaiklay, S., Phongpaichit, S., Borwornwiriyapan, K., and Sakayaroj, J., Tetrahedron, 2014, vol. 70, pp. 5148-5152. https://doi.org/10.1016/j.tet.2014.05.105

231. Ivanets, E., Yurchenko, A., Smetanina, O., Rasin, A., Zhuravleva, O., Pivkin, M., Popov, R., von Amsberg, G., Afiyatullov, S., and Dyshlovoy, S., Mar. Drugs, 2018, vol. 16, p. 232. https://doi.org/10.3390/md16070232

232. Xu, L.-L., Hai, P., Zhang, S.-B., Xiao, J.-F., Gao, Y., Ma, B.-J., Fu, H.-Y., Chen, Y.-M., and Yang, X.-L., J. Nat. Prod., 2019, vol. 82, pp. 221-231. https://doi.org/10.1021/acs.jnatprod.8b00589

233. Khalil, Z.G., Huang, X., Raju, R., Piggott, A.M., and Capon, R.J., J. Org. Chem., 2014, vol. 79, pp. 87008705 . https://doi.org/10.1021/jo501501z

234. Yang, S.-Q., Li, X.-M., Li, X., Chi, L.-P., and Wang, B.-G., Mar. Drugs, 2018, vol. 16, p. 114. https://doi.org/10.3390/md16040114

235. Lacey, H.J., Vuong, D., Pitt, J.I., Lacey, E., and Piggott, A.M., Aust. J. Chem., 2016, vol. 69, pp. 152-160. https://doi.org/10.1071/CH15488

236. Johnson, J.H., Phillipson, D.W., and Kahle, A.D., J. Antibiot., 1989, vol. 42, pp. 1184-1185. https://doi.org/10.7164/antibiotics.42.1184

237. Limbadri, S., Luo, X., Lin, X., Liao, S., Wang, J., Zhou, X., Yang, B., and Liu, Y., Molecules, 2018, 
vol. 23, p. 2379.

https://doi.org/10.3390/molecules23092379

238. Takahashi, C., Matsushita, T., Doi, M., Minoura, K., Shingu, T., Kumeda, Y., and Numata, A., J. Chem. Soc., Perkin Trans. 1, 1995, pp. 2345-2353. https://doi.org/10.1039/P19950002345

239. Rank, C., Phipps, R.K., Harris, P., Frisvad, J.C., Gotfredsen, C.H., and Larsen, T.O., Tetrahedron Lett., 2006, vol. 47, pp. 6099-6102.

https://doi.org/10.1016/j.tetlet.2006.06.086

240. Afiyatullov, S.Sh., Zhuravleva, O.I., Antonov, A.S., Berdyshev, D.V., Pivkin, M.V., Denisenko, V.A., Popov, R.S., Gerasimenko, A.V., von Amsberg, G., Dyshlovoy, S.A., Leshchenko, E.V., and Yurchenko, A.N., J. Antibiot., 2018, vol. 71, pp. 846-853. https://doi.org/10.1038/s41429-018-0072-9

241. Zhou, R., Liao, X., Li, H., Li, J., Feng, P., Zhao, B., and Xu, S., Front. Chem., 2018, vol. 6, p. 212. https://doi.org/10.3389/fchem.2018.00212

242. Zheng, C.-J., Bai, M., Zhou, X.-M., Huang, G.-L., Shao, T.-M., Luo, Y.-P., Niu, Z.-G., Niu, Y.-Y., Chen, G.-Y., and Han, C.-R., J. Nat. Prod., 2018, vol. 81 , pp. $1045-1049$.

https://doi.org/10.1021/acs.jnatprod.7b00673

243. Elsbaey, M., Tanaka, C., and Miyamoto, T., Phytochemistry Lett., 2019, vol. 32, pp. 70-76. https://doi.org/10.1016/j.phytol.2019.04.023

244. Chen, Y., Liu, Z., Huang, Y., Liu, L., He, J., Wang, L., Yuan, J., and She, Z., J. Nat. Prod., 2019, vol. 82, pp. 1752-1758. https://doi.org/10.1021/acs.jnatprod.8b00918

245. Wijeratne, E.M.K., He, H., Franzblau, S.G., Hoffman, A.M., and Gunatilaka, A.A.L., J. Nat. Prod., 2013, vol. 76, pp. 1860-1865. https://doi.org/10.1021/np400391p

246. Wang, P., Cui, Y., Cai, C., Chen, H., Dai, Y., Chen, P., Kong, F., Yuan, J., Song, X., Mei, W., and Dai, H., Mar. Drugs, 2018, vol. 17, p. 4. https://doi.org/10.3390/md17010004

247. Suzuki, S., Hosoe, T., Nozawa, K., Kawai, K., Yaguchi, T., and Udagawa, S., J. Nat. Prod., 2000, vol. 63, pp. 768-772. https://doi.org/10.1021/np990371x

248. Bao, J., Zhai, H., Zhu, K., Yu, J.-H., Zhang, Y., Wang, Y., Jiang, C.-S., Zhang, X., Zhang, Y., and Zhang, H., Mar. Drugs, 2018, vol. 16, p. 174. https://doi.org/10.3390/md16050174

249. Alfatafta, A.A., Gloer, J.B., Scott, J.A., and Malloch, D., J. Nat. Prod., 1994, vol. 57, pp. 1696-1702. https://doi.org/10.1021/np50114a012

250. Jiao, R.H., Xu, S., Liu, J.Y., Ge, H.M., Ding, H., Xu, C., Zhu, H.L., and Tan, R.X., Org. Lett., 2006, vol. 8, pp. 5709-5712. https://doi.org/10.1021/ol062257t

251. TePaske, M.R., Gloer, J.B., Wicklow, D.T., and Dowd, P.F., Tetrahedron Lett., 1991, vol. 32, pp. 56875690 . https://doi.org/10.1016/S0040-4039(00)93530-5

252. Ge, H.M., Shen, Y., Zhu, C.H., Tan, S.H., Ding, H., Song, Y.C., and Tan, R.X., Phytochemistry, 2008, vol. 69 , pp. $571-576$.

https://doi.org/10.1016/j.phytochem.2007.07.014
253. Banert, K. and Tantillo, D.J., Nat. Prod. Res., 2019, vol. 33, pp. 3011-3015. https://doi.org/10.1080/14786419.2018.1509330

254. Tao, H., Li, Y., Lin, X., Zhou, X., Dong, J., Liu, Y., and Yang, B., Nat. Prod. Commun., 2018, vol. 13, pp. $1629-1631$. https://doi.org/10.1177/1934578X1801301214

255. Choi, B.-K., Trinh, P.T.H., Lee, H.-S., Choi, B.-W., Kang, J.S., Ngoc, N.T.D., Van, T.T.T., and Shin, H.J., Mar. Drugs, 2019, vol. 17, 346. https://doi.org/10.3390/md17060346

256. Bladt, T., Dürr, C., Knudsen, P., Kildgaard, S., Frisvad, J., Gotfredsen, C., Seiffert, M., and Larsen, T., Molecules, 2013, vol. 18, pp. 14629-14650. https://doi.org/10.3390/molecules181214629

257. Tan, Y., Yang, B., Lin, X., Luo, X., Pang, X., Tang, L., Liu, Y., Li, X., and Zhou, X., J. Nat. Prod., 2018, vol. 81, pp. 92-97. https://doi.org/10.1021/acs.jnatprod.7b00698

258. Fang, W., Lin, X., Zhou, X., Wan, J., Lu, X., Yang, B., Ai, W., Lin, J., Zhang, T., Tu, Z., and Liu, Y., Med. Chem. Commun., 2014, vol. 5, pp. 701-705. https://doi.org/10.1039/C3MD00371J

259. Belofsky, G., Jensen, P., Renner, M., and Fenical, W., Tetrahedron, 1998, vol. 54, pp. 1715-1724. https://doi.org/10.1016/S0040-4020(97)10396-9

260. Deng, Q., Li, G., Sun, M., Yang, X., and Xu, J., Nat. Prod. Res., 2018, vol. 34, pp. 1404-1408. https://doi.org/10.1080/14786419.2018.1512993

261. Zhou, J., Li, G., Deng, Q., Zheng, D., Yang, X., and Xu, J., Nat. Prod. Res., vol. 32, 2018, pp. 2968-2972. https://doi.org/10.1080/14786419.2017.1395431

262. Hamasaki, T., Nagayama, K., and Hatsuda, Y., Agric. Biol. Chem., 1978, vol. 42, pp. 37-40. https://doi.org/10.1080/00021369.1978.10862919

263. Li, X.-D., Li, X.-M., Xu, G.-M., Zhang, P., and Wang, B.-G., J. Nat. Prod., 2015, vol. 78, pp. 844-849. https://doi.org/10.1021/acs.jnatprod.5b00004

264. Wang, P., Yu, J.-H., Zhu, K., Wang, Y., Cheng, Z.-Q., Jiang, C.-S., Dai, J.-G., Wu, J., and Zhang, H., Fitoterapia, 2018, vol. 127, pp. 322-327. https://doi.org/10.1016/j.fitote.2018.02.031

265. Yao, Q., Wang, J., Zhang, X., Nong, X., Xu, X., and Qi, S., Mar. Drugs, 2014, vol. 12, pp. 5902-5915. https://doi.org/10.3390/md12125902

266. Qiu, L., Wang, P., Liao, G., Zeng, Y., Cai, C., Kong, F., Guo, Z., Proksch, P., Dai, H., and Mei, W., Mar. Drugs, 2018, vol. 16, p. 108. https://doi.org/10.3390/md16040108

267. Xing, C.-P., Xie, C.-L., Xia, J.-M., Liu, Q.-M., Lin, W.-X., Ye, D.-Z., Liu, G.-M., and Yang, X.-W., Mar. Drugs, 2019, vol. 17, p. 507. https://doi.org/10.3390/md17090507

268. Amagata, T., Doi, M., Tohgo, M., Minoura, K., and Numata, A., Chem. Commun., 1999, pp. 1321-1322. https://doi.org/10.1039/a903840j

269. Amagata, T., Tanaka, M., Yamada, T., Doi, M., Minoura, K., Ohishi, H., Yamori, T., and Numata, A., J. Nat. Prod., 2007, vol. 70, pp. 1731-1740. https://doi.org/10.1021/np070165m 
270. Guo, Y.-W., Gong, B.-Q., Yuan, J., Li, H.-J., Mahmud, T., Huang, Y., Li, J.-F., Yang, D.-P., and Lan, W.-J., J. Nat. Prod., 2019, vol. 83, pp. 79-87. https://doi.org/10.1021/acs.jnatprod.9b00710

271. Yang, B., He, Y., Lin, S., Zhang, J., Li, H., Wang, J., Hu, Z., and Zhang, Y., J. Nat. Prod., 2019, vol. 82, pp. $1923-1929$. https://doi.org/10.1021/acs.jnatprod.9b00305

272. Evidente, A., Sparapano, L., Motta, A., Giordano, F., Fierro, O., and Frisullo, S., Phytochemistry, 1996, vol. 42, pp. $1541-1546$. https://doi.org/10.1016/0031-9422(96)00206-3

273. Yan, T., Guo, Z., Jiang, R., Wei, W., Wang, T., Guo, Y., Song, Y., Jiao, R., Tan, R., and Ge, H., Planta Med., 2013, vol. 79, pp. 348-352. https://doi.org/10.1055/s-0032-1328260

274. Lallemand, B., Masi, M., Maddau, L., De Lorenzi, M., Dam, R., Cimmino, A., Moreno y Banuls, L., Andolfi, A., Kiss, R., Mathieu, V., and Evidente, A., Phytochemistry Lett., 2012, vol. 5, pp. 770-775. https://doi.org/10.1016/j.phytol.2012.08.011

275. Li, Y., Ye, D., Shao, Z., Cui, C., and Che, Y., Mar. Drugs, 2012, vol. 10, pp. 497-508. https://doi.org/10.3390/md10020497

276. Yu, H.-B., Wang, X.-L., Zhang, Y.-X., Xu, W.-H., Zhang, J.-P., Zhou, X.-Y., Lu, X.-L., Liu, X.-Y., and Jiao, B.-H., J. Nat. Prod., 2018, vol. 81, pp. 15531560. https://doi.org/10.1021/acs.jnatprod.8b00039

277. Lu, X.-L., Liu, J.-T., Liu, X.-Y., Gao, Y., Zhang, J., Jiao, B.-H., and Zheng, H., J. Antibiot., 2014, vol. 67, pp. $171-174$. https://doi.org/10.1038/ja.2013.104

278. Li, X.-D., Li, X., Li, X.-M., Xu, G.-M., Liu, Y., and Wang, B.-G., Mar. Drugs, 2018, vol. 16, p. 440. https://doi.org/10.3390/md16110440

279. Ratnaweera, P.B., Williams, D.E., de Silva, E.D., Wijesundera, R.L.C., Dalisay, D.S., and Andersen, R.J, Mycology, 2014, vol. 5, pp. 23-28. https://doi.org/10.1080/21501203.2014.892905

280. Yang, M.-H., Li, T.-X., Wang, Y., Liu, R.-H., Luo, J., and Kong, L.-Y., Fitoterapia, 2017, vol. 116, pp. 72-76. https://doi.org/10.1016/j.fitote.2016.11.008

281. Amagata, T., Amagata, A., Tenney, K., Valeriote, F.A., Lobkovsky, E., Clardy, J., and Crews, P., Org. Lett., 2003, vol. 5, pp. 4393-4396. https://doi.org/10.1021/ol0356800

282. Kong, F.-D., Huang, X.-L., Ma, Q.-Y., Xie, Q.-Y., Wang, P., Chen, P.-W., Zhou, L.-M., Yuan, J.-Z., Dai, H.-F., Luo, D.-Q., and Zhao, Y.-X., J. Nat. Prod., 2018, vol. 81, pp. 1869-1876. https://doi.org/10.1021/acs.jnatprod.8b00382

283. Springer, J.P., Dorner, J.W., Cole, R.J., and Cox, R.H., J. Org. Chem., 1979, vol. 44, pp. 48524854. https://doi.org/10.1021/jo00394a023

284. Li, H.-L., Li, X.-M., Li, X., Yang, S.-Q., and Wang, B.-G., Phytochem. Lett., 2019, vol. 32, pp. 138142. https://doi.org/10.1016/j.phytol.2019.05.017

285. Li, G.-Y., Li, B.-G., Yang, T., Yin, J.-H., Qi, H.-Y., Liu, G.-Y., and Zhang, G.-L., J. Nat. Prod., 2005, vol. 68 , pp. $1243-1246$.

https://doi.org/10.1021/np0501738
286. Fukuda, T., Kurihara, Y., Kanamoto, A., and Tomoda, H., J. Antibiot., 2014, vol. 67, pp. 593-595.

https://doi.org/10.1038/ja.2014.46

287. Hamed, A., Abdel-Razek, A.S., Omran, D.A., ElMetwally, M.M., El-Hosari, D.G., Frese, M., Soliman, H.S.M., Sewald, N., and Shaaban, M., Nat. Prod. Res., 2020, vol. 34, pp. 965-974. https://doi.org/10.1080/14786419.2018.1544977

288. Shaaban, M., El-Metwally, M.M., Abdel-Razek, A.A., and Laatsch, H., Nat. Prod. Res., 2018, vol. 32, pp. 2437-2446. https://doi.org/10.1080/14786419.2017.1419230

289. Hamed, A., Abdel-Razek, A., Frese, M., Stammler, H., El-Haddad, A., Ibrahim, T., Sewald, N., and Shaaban, M., Molecules, 2018, vol. 23, p. 299. https://doi.org/10.3390/molecules23020299

290. Fan, S.-Q., Xie, C.-L., Xia, J.-M., Xing, C.-P., Luo, Z.-H., Shao, Z., Yan, X.-J., He, S., and Yang, X.-W., Org. Biomol. Chem., 2019, vol. 17, pp. 5925-5928. https://doi.org/10.1039/C9OB01159E

291. Li, W.-H., Chang, S.-T., Chang, S.-C., and Chang, H.-T., Nat. Prod. Res., 2008, vol. 22, pp. 10851093. https://doi.org/10.1080/14786410802267510

292. Rosenberger, M. and Neukom, C., J. Org. Chem., 1982, vol. 47, pp. 1782-1785. https://doi.org/10.1021/jo00348a043

293. Gao, H., Hong, K., Chen, G.-D., Wang, C.-X., Tang, J.-S., Yu, Y., Jiang, M.-M., Li, M.-M., Wang, N.-L., and Yao, X.-S., Magn. Reson. Chem., 2009, vol. 48, pp. 38-43. https://doi.org/10.1002/mrc.2536

294. Talapatra, S.K., Bhaumik, A., and Talapatra, B., Phytochemistry, 1992, vol. 31, pp. 2431-2434. https://doi.org/10.1016/0031-9422(92)83293-8

295. Tong, Z.-B., Cui, X.-H., Wang, J., Zhang, C.-L., Zhang, Y.-Y., and Ren, Z.-J., Nat. Prod. Res., 2017, vol. 31, pp. 2564-2567. https://doi.org/10.1080/14786419.2017.1320785

296. Chen, S. and Snyder, J.K., J. Org. Chem., 1989, vol. 54, pp. 3679-3689. https://doi.org/10.1021/jo00276a033

297. Gao, S.-S., Li, X.-M., Li, C.-S., Proksch, P., and Wang, B.-G., Bioorg. Med. Chem. Lett., 2011, vol. 21, pp. 2894-2897. https://doi.org/10.1016/j.bmcl.2011.03.076

298. Igarashi, Y., Sekine, A., Fukazawa, H., Uehara, Y., Yamaguchi, K., Endo, Y., Okuda, T., Furumai, T., and Oki, T., J. Antibiot., 2002, vol. 55, pp. 371-376. https://doi.org/10.7164/antibiotics.55.371

299. Xie, C.-L., Zhang, D., Xia, J.-M., Hu, C.-C., Lin, T., Lin, Y.-K., Wang, G.-H., Tian, W.-J., Li, Z.-P., Zhang, X.-K., Yang, X.-W., and Chen, H.-F., Mar. Drugs, 2019, vol. 17, p. 178. https://doi.org/10.3390/md17030178

300. Abdel-Razek, A.S., Hamed, A., Frese, M., Sewald, N., and Shaaban, M., Steroids, 2018, vol. 138, pp. 21-25. https://doi.org/10.1016/j.steroids.2018.06.005

301. Kaleem, S., Ge, H., Yi, W., Zhang, Z., and Wu, B., Nat. Prod. Res., 2019. https://doi.org/10.1080/14786419.2019.1680669

302. Bayman, P., Baker, J.L., Doster, M.A., Michailides, T.J., and Mahoney, N.E., Appl. Environ. Microbiol., 2002, vol. 68 , pp. $2326-2329$.

https://doi.org/10.1128/AEM.68.5.2326-2329.2002 
303. Wang, Y., Wang, L., Liu, F., Wang, Q., Selvaraj, J., Xing, F., Zhao, Y., and Liu, Y., Toxins, 2016, vol. 8, p. 83. https://doi.org/10.3390/toxins8030083

304. Sargent, M.V. and Stransky, P.O., J. Chem. Soc., Perkin Trans., 1982, pp. 1605-1610. https://doi.org/10.1039/p19820001605

305. Wang, J., Lu, Z., Liu, P., Wang, Y., Li, J., Hong, K., and Zhu, W., Planta Med., 2012, vol. 78, pp. 18611866.

https://doi.org/10.1055/s-0032-1315395

306. Budde, K., Quella, F., Mathes, A., Melchior, W., Müller, H., Nuyken, O., and Spiegel, S., Angew. Makromol. Chem., 1992, vol. 194, pp. 103-117. https://doi.org/10.1002/apmc.1992.051940109

307. Zhao, Y., Liu, D., Proksch, P., Zhou, D., and Lin, W., Phytochemistry, 2018, vol. 155, pp. 61-68. https://doi.org/10.1016/j.phytochem.2018.07.017

308. Liu, Y., Ding, L., Fang, F., and He, S., Nat. Prod. Res., 2019, vol. 33, pp. 2466-2470. https://doi.org/10.1080/14786419.2018.1452012

309. Li, T.-X., Yang, M.-H., Wang, X.-B., Wang, Y., and Kong, L.-Y., J. Nat. Prod., 2015, vol. 78, pp. 25112520. https://doi.org/10.1021/acs.jnatprod.5b00008

310. Ding, L., Xu, P., Li, T., Liao, X., He, S., and Xu, S., Nat. Prod. Res., vol. 33, 2019, pp. 3404-3408. https://doi.org/10.1080/14786419.2018.1480622

311. Cai, J., Zhou, X.-M., Yang, X., Tang, M.-M., Liao, Q.-Y., Meng, B.-Z., Liao, S., and Chen, G.-Y., Bioorg. Chem., 2019, p. 103362. https://doi.org/10.1016/j.bioorg.2019.103362

312. Bai, M., Huang, G.-L., Mei, R.-Q., Wang, B., Luo, Y.-P., Nong, X.-H., Chen, G.-Y., and Zheng, C.-J., Mar. Drugs, 2019, vol. 17, p. 433. https://doi.org/10.3390/md17080433

313. Zhou, X.-M., Zheng, C.-J., Song, X.-M., Tang, M.-M., Yang, J.-Y., Yang, X., Peng, S.-J., Cai, J., and Chen, G.-Y., Fitoterapia, 2019, vol. 139, p. 104400. https://doi.org/10.1016/j.fitote.2019.104400

314. Wu, J.-T., Zheng, C.-J., Zhang, B., Zhou, X.-M., Zhou, Q., Chen, G.-Y., Zeng, Z.-E., Xie, J.-L., Han, C.-R., and Lyu, J.-X., Nat. Prod. Res., 2019, vol. 33 , pp. 34-40. https://doi.org/10.1080/14786419.2018.1431634

315. Kongyen, W., Rukachaisirikul, V., Phongpaichit, S., and Sakayaroj, J., Nat. Prod. Res., 2015, vol. 29, pp. 1995-1999. https://doi.org/10.1080/14786419.2015.1022542

316. Rao, K.V., Sadhukhan, A.K., Veerender, M., Ravikumar, V., Mohan, E.V.S., Dhanvantri, S.D., Sitaramkumar, M., Moses Babu, J., Vyas, K., and Om Reddy, G., Chem. Pharm. Bull., 2000, vol. 48, pp. 559-562. https://doi.org/10.1248/cpb.48.559

317. Sun, W., Luan, S., Qi, C., Tong, Q., Yan, S., Li, H., and Zhang, Y., Cell Commun. Signal., 2019, vol. 17, 111. https://doi.org/10.1186/s12964-019-0425-4

318. Zeng, Q., Zhong, W.-M., Chen, Y.-C., Xiang, Y., Chen, X.-Y., Tian, X.-P., Zhang, W.-M., Zhang, S., and Wang, F.-Z., Nat. Prod. Res., 2020, vol. 34, pp. 1984-1991. https://doi.org/10.1080/14786419.2019.1569658

319. Sun, Y., Liu, J., Li, L., Gong, C., Wang, S., Yang, F., Hua, H., and Lin, H., Bioorg. Med. Chem. Lett., 2018, vol. 28 , pp. $315-318$.

https://doi.org/10.1016/j.bmcl.2017.12.049
320. Parvatkar, R.R., D'Souza, C., Tripathi, A., and Naik, C.G., Phytochemistry, 2009, vol. 70, pp. 128-132. https://doi.org/10.1016/j.phytochem.2008.10.017

321. Zhu, J.X., Ding, L., and He, S., Nat. Prod. Res., 2019, vol. 33, pp. 1191-1195. https://doi.org/10.1080/14786419.2018.1465423

322. Ding, L., Li, T., Liao, X., He, S., and Xu, S., J. Antibiot., 2018, vol. 71, pp. 902-904. https://doi.org/10.1038/s41429-018-0079-2

323. Blair, L.M. and Sperry, J., J. Nat. Prod., 2013, vol. 76, pp. 794-812. https://doi.org/10.1021/np400124n

324. Wermuth, C.G., MedChemComm, 2011, vol. 2, pp. 935-941. https://doi.org/10.1039/C1MD00074H

325. Zhang, Y.-X., Yu, H.-B., Xu, W.-H., Hu, B., Guild, A., Zhang, J.-P., Lu, X.-L., Liu, X.-Y., and Jiao, B.-H., J. Nat. Prod., 2019, vol. 82, pp. 3089-3095. https://doi.org/10.1021/acs.jnatprod.9b00700

326. Hayashi, H., Mukaihara, M., Murao, S., Arai, M., Lee, A.Y., and Clardy, J., Biosci., Biotechnol., Biochem., 1994, vol. 58, pp. 334-338. https://doi.org/10.1271/bbb.58.334

327. Cheng, Z., Xu, W., Liu, L., Li, S., Yuan, W., Luo, Z., Zhang, J., Cheng, Y., and Li, Q., Mar. Drugs, 2018, vol. 16 , p. 358 .

https://doi.org/10.3390/md16100358

328. Giromini, C., Rebucci, R., Fusi, E., Rossi, L., Saccone, F., and Baldi, A., Cell Biol. Toxicol., 2016, vol. 32, pp. 249-258.

https://doi.org/10.1007/s10565-016-9332-2

329. Rottkord, U., Röhl, C., Ferse, I., Schulz, M.-C., Rückschloss, U., Gekle, M., Schwerdt, G., and Humpf, H.-U., Arch. Toxicol., 2017, vol. 91, pp. 14611471. https://doi.org/10.1007/s00204-016-1799-3

330. Cramer, B., Harrer, H., Nakamura, K., Uemura, D., and Humpf, H.-U., Bioorg. Med. Chem., 2010, vol. 18, pp. 343-347.

https://doi.org/10.1016/j.bmc.2009.10.050

331. Brown, E.D. and Wright, G.D., Nature, 2016, vol. 529, pp. 336-343. https://doi.org/10.1038/nature 17042

332. Lewis, K., Nature, 2012, vol. 485, pp. 439-440. https://doi.org/10.1038/485439a

333. Lewis, K., Nat. Rev. Drug Discov., 2013, vol. 12, pp. 371-387. https://doi.org/10.1038/nrd3975

334. Cameron, A.J., Davison, E.K., An, C., Stubbing, L.A., Dunbar, P.R., Harris, P.W.R., and Brimble, M.A., J. Org. Chem., 2020, vol. 85, pp. 1401-1406. https://doi.org/10.1021/acs.joc.9b02348

335. Lai, Y., Zhang, N., Zhang, Y., Chen, J.-H., and Yang, Z., Org. Lett., 2018, vol. 20, pp. 4298-4301. https://doi.org/10.1021/acs.orglett.8b01733

336. Wolfender, J.-L., Litaudon, M., Touboul, D., and Queiroz, E.F., Nat. Prod. Rep., 2019, vol. 36, pp. 855868.

https://doi.org/10.1039/C9NP00004F

Translated by A. Levina 